\title{
Geometry of quantum phase transitions
}

\author{
Angelo Carollo ${ }^{\mathrm{a}, \mathrm{b}}$, Davide Valenti ${ }^{\mathrm{a}}$, Bernardo Spagnolo ${ }^{\mathrm{a}, \mathrm{b}, \mathrm{c}, *}$ \\ a Dipartimento di Fisica e Chimica 'Emilio Segrè', Group of Interdisciplinary Theoretical Physics, Università di Palermo, Viale delle \\ Scienze, Ed. 18, I-90128 Palermo, Italy \\ ${ }^{\mathrm{b}}$ Radiophysics Department, Lobachevsky State University of Nizhni Novgorod, 23 Gagarin Avenue, Nizhni Novgorod 603950, Russia \\ ${ }^{\mathrm{c}}$ Istituto Nazionale di Fisica Nucleare, Sezione di Catania, Via S. Sofia 64, I-90123 Catania, Italy
}

\section{A R T I C L E I N F O}

\section{Article history:}

Received 5 November 2019

Accepted 6 November 2019

Available online 15 November 2019

Editor: I. Procaccia

\section{Keywords:}

Quantum geometric information

Geometric phase

Quantum phase transitions

Dissipative phase transitions

Quantum metrology

\begin{abstract}
A B S T R A C T
In this article we provide a review of geometrical methods employed in the analysis of quantum phase transitions and non-equilibrium dissipative phase transitions. After a pedagogical introduction to geometric phases and geometric information in the characterisation of quantum phase transitions, we describe recent developments of geometrical approaches based on mixed-state generalisation of the Berry-phase, i.e. the Uhlmann geometric phase, for the investigation of non-equilibrium steady-state quantum phase transitions (NESS-QPTs ). Equilibrium phase transitions fall invariably into two markedly non-overlapping categories: classical phase transitions and quantum phase transitions, whereas in NESS-QPTs this distinction may fade off. The approach described in this review, among other things, can quantitatively assess the quantum character of such critical phenomena. This framework is applied to a paradigmatic class of lattice Fermion systems with local reservoirs, characterised by Gaussian non-equilibrium steady states. The relations between the behaviour of the geometric phase curvature, the divergence of the correlation length, the character of the criticality and the gap - either Hamiltonian or dissipative - are reviewed.
\end{abstract}

(C) 2019 Elsevier B.V. All rights reserved.

\section{Contents}

1. Introduction

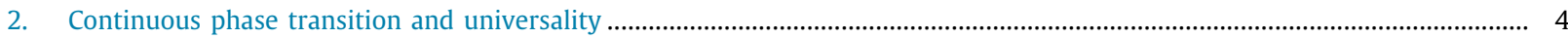

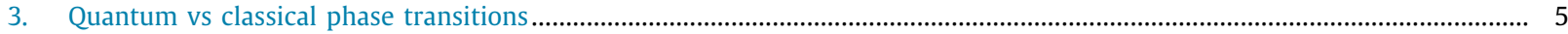

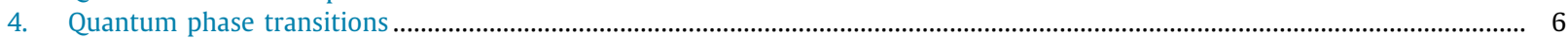

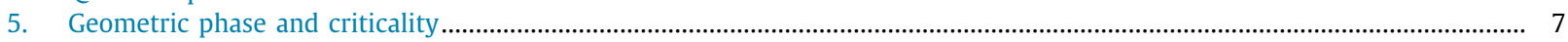

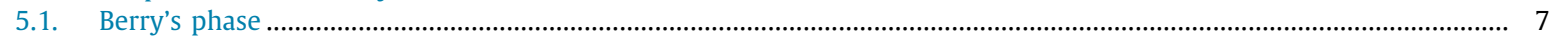

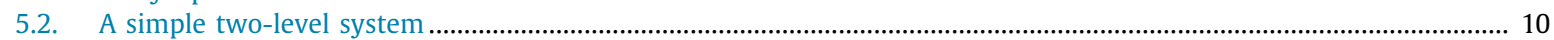

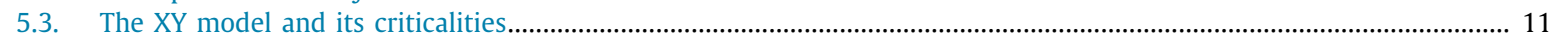

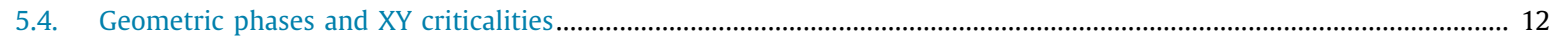

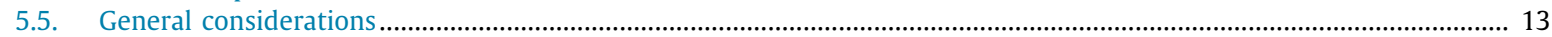

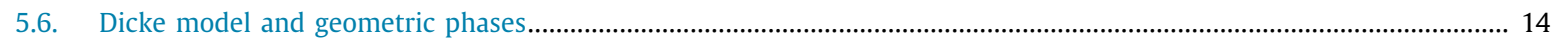

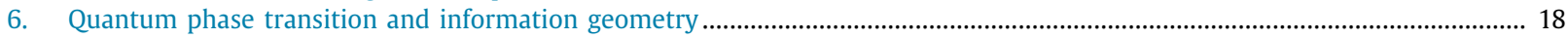

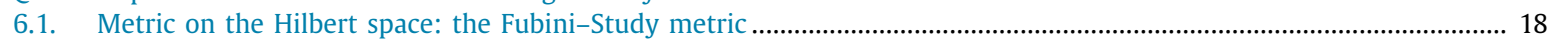

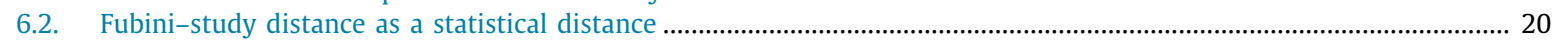

* Corresponding author at: Dipartimento di Fisica e Chimica 'Emilio Segrè', Group of Interdisciplinary Theoretical Physics, Università di Palermo, Viale delle Scienze, Ed. 18, I-90128 Palermo, Italy.

E-mail address: bernardo.spagnolo@unipa.it (B. Spagnolo). 
6.3. Fubini-study metric and quantum phase transitions 20

6.4. Quantum phase transition and super-extensitivity of the quantum geometric tensor .................................................. 21

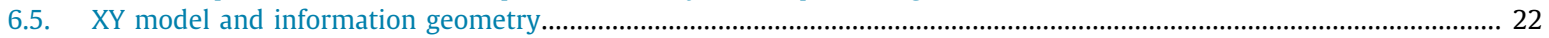

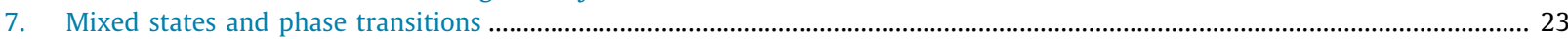

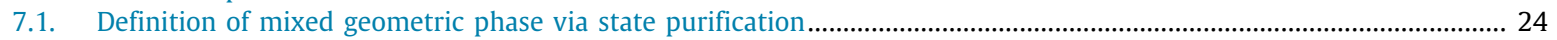

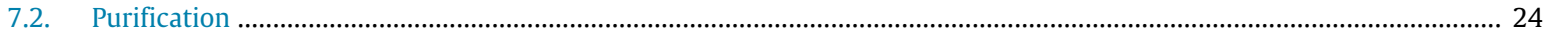

7.3. Parallel transport of density matrices and Uhlmann geometric phase .................................................................... 25

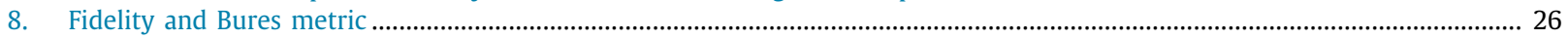

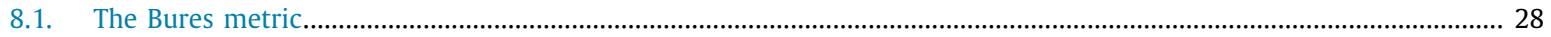

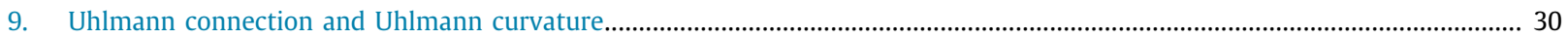

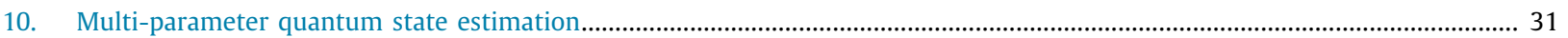

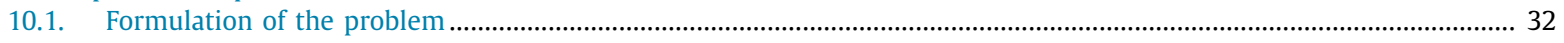

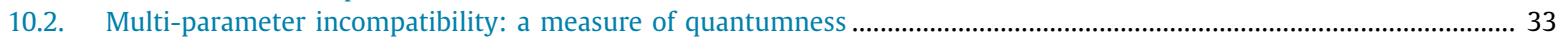

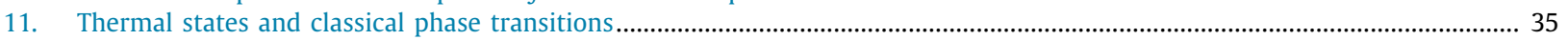

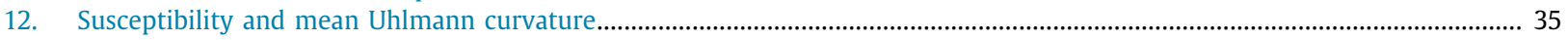

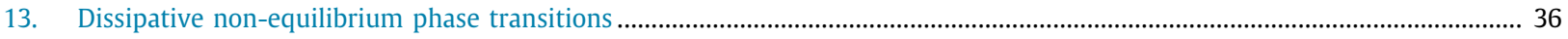

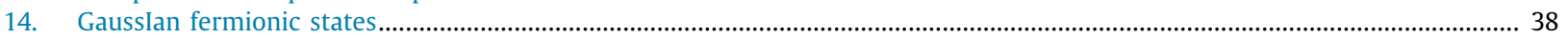

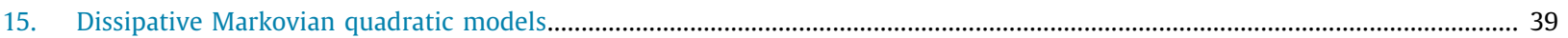

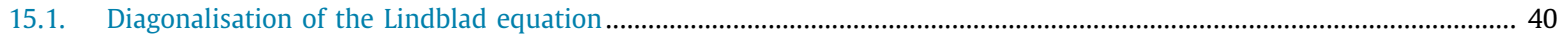

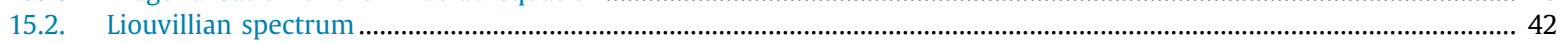

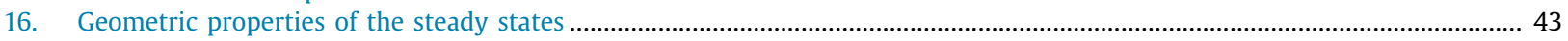

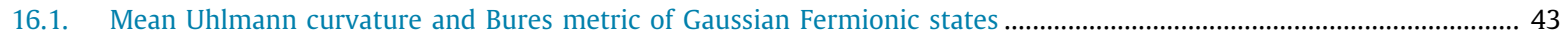

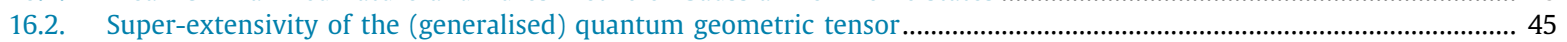

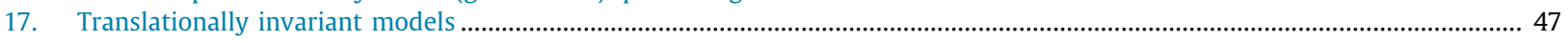

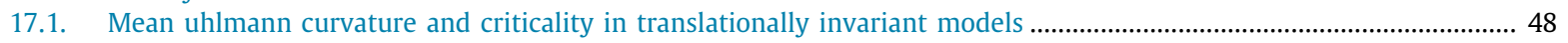

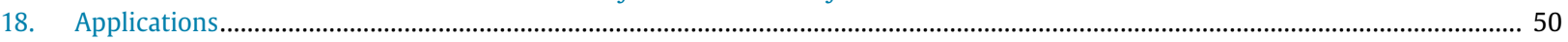

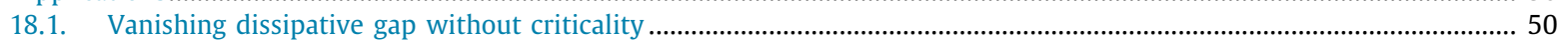

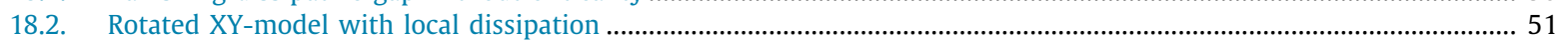

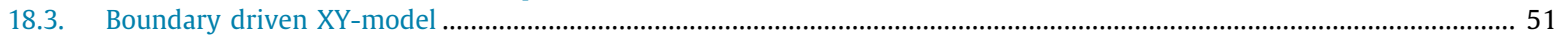

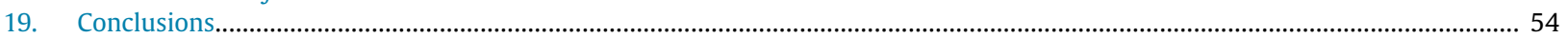

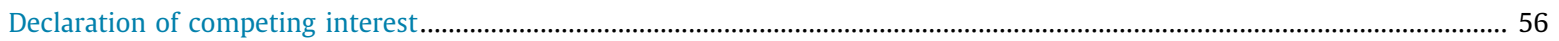

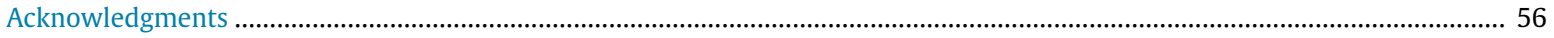

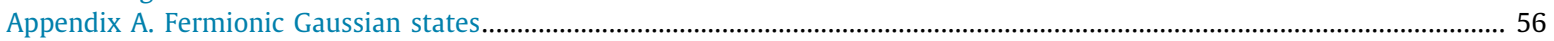

A.1. Symmetric logarithmic derivative for Fermionic Gaussian states ........................................................................ 57

Appendix B. Spectral properties of Quadratic Liouvillians .................................................................................................. 59

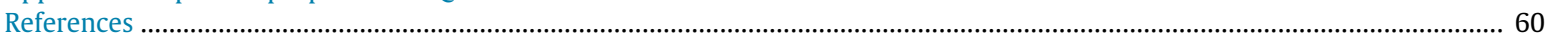

\section{Introduction}

Several systems manifest phase transitions as the temperature or other external parameters are modified, and are characterised by a qualitative changes in the system properties. Examples of phase transitions range from our mundane experience of ice melting or the loss of ferromagnetism in iron to the far more exotic superfluid Mott-insulator phase transitions in optical lattices [1].

Thermal phase transitions, i.e. phase transitions that occur at finite temperature, are characterised by a macroscopic order, such as magnetisation or crystal structure, which gets destroyed by the thermal fluctuations [2-6]. At zero temperature, a different type of phase transitions can take place as a non-thermal parameter, such as magnetic field, pressure or chemical composition, is changed. The result is an order in the ground state properties of the system which is destroyed exclusively by quantum fluctuations. Competition between different terms of the system's Hamiltonian, and the ordering thereof, are responsible for such fluctuations, which are a macroscopic manifestation of the Heisenberg uncertainty principle [7-12].

In the past few decades, the studies of phase transitions at zero and finite temperature has been fuelled by the formidable success of Ginzburg-Landau-Wilson theories, local ordering, spontaneous symmetry breaking and the renormalisation group [13-16] in spelling out the nature of a wide variety of critical behaviours.

Such a standard approach has been recently parallelled by the development of a framework emerging from the cross-pollination of quantum information theory, information geometry and metrology. This approach is differentialgeometric in essence and relies on two intertwined concepts: geometric phase and fidelity. The idea is to explore the critical features of many-body systems through the geometrical properties of its equilibrium state. The intuition behind it is relatively straightforward. Phase transitions are characterised by dramatic differences in some system's observable under infinitesimal variation of a control parameter, which are embodied in major structural changes of the state of the system. One could compare the states associated to infinitesimally close values of the parameters by some "similarity" function, or distance function. One would then be able to reveal these major structural changes in the state through the 
singular behaviour of such a distance function. The Uhlmann fidelity [17-24] provides a quantitative measure of such a distance, called the Bures metric [25].

Alternatively, in a quantum phase transitions (QPT), one may look at these dramatic changes as the manifestation of level crossings, occurring at the thermodynamic limit, which involve ground state and low lying part of the energy spectrum. In much the same way as mass-energy singularity bends the geometry of the space-time, or a Dirac monopole twists the topology of the field configuration around it [26], a level crossing bends the geometry of the ground-state manifold in a singular way. A quantitative account of this effect is provided by the geometric phase [27-30], or more precisely, its infinitesimal counterpart, the Berry curvature. Driving the system close to or around these singularities results in dramatic effects on the state geometry, picked up by a quantum state in the form of geometric phase instabilities [31-33]. Geometric phases are at the core of the characterisation of topological phase transitions [34-36], and have been employed in the description and detection of QPT, both theoretically [31,32,37-40] and experimentally [41].

Singular curvature and singular metric are two complementary manifestations of the same exceptional behaviour of the quantum state arising across phase transitions [42]. These quantities are a direct expression of the geometry of the state manifold and require no a-priori notions of order parameters, or symmetry breaking. They do not even require a detailed knowledge on the dynamics of the physical model, (e.g. the Hamiltonian), but only on its kinematics (i.e. the state of the system, and its dependence on parameters). This means that this conceptual framework can be freed from the boundaries of the standard Ginzburg-Landau-Wilson paradigm, and can be used to study a wide range of critical behaviours where a notion of local order does not hold, or cannot be easily identified.

This motivates the employment of geometric phases in the investigation of critical properties in a plethora of different scenarios [31-33,37-115].

The idea that QPTs could be explored through the Berry phase properties was first proposed and applied in the prototypical XY spin-1/2 chain $[31,32,37,39,40,57,65,76]$ and extended to many other many-body systems, such as the Dicke model [38,44], the Lipkin-Meshkov-Glick model [43,60,72], Yang-Baxter spin-1/2 model [49,58], quasi free-Fermion systems [47,53,56,85,102], interacting Fermion models [51,63,68,77,79,98,116], in ultracold atoms [73,74,91], in spin chains with long range interactions [70,81], in cluster models [106], in the spin-boson model [114], in the 1D compassmodel [59,96], and in connection to spin-crossover phenomena [55]. The critical properties of the geometric phase has also been studied in few-body systems interacting with critical chains [66,67,78,87,92,97,100], in non-Hermitian critical systems [86,88], in connection to dynamical phase transitions [95,104], in the characterisation of topological phase transitions [83,84,94,99,101,103,107,117-121], in quenched systems [61,64,93], in non-equilibrium phase transitions [80,82,110,115,122], in connection with entanglement [75,89], and renormalisation group [90].

Beyond zero-temperature and thermal phase transitions, a novel type of criticalities have recently emerged in the context of non-equilibrium steady states (NESSes) of open-driven dissipative systems [123-153]. In this context, one considers a quantum many body-system coupled to external reservoirs whose dynamics may be described effectively in terms of a Liouvillian master equation $[154,155]$. Under suitable conditions, the open system dynamics reaches a (possibly unique) non-equilibrium steady state. The dynamical and static properties of the NESSes characterise the type of phases in which the systems lies.

In this setting, NESS quantum phase transitions (NESS-QPTs) are revealed as dramatic structural changes of the Liouvillian steady state upon a slight modification of tuneable external parameters. The analogy with equilibrium phase transitions is straightforward. QPTs are understood through the properties of the (unique) ground state of the Hamiltonian governing the dynamics of the system. Phase diagrams and criticalities are determined by the low-lying spectrum of excitations of the system's Hamiltonian. Similarly, in dynamics governed by a Liouvillian master equation, observable macroscopical changes in the properties of quantum systems are revealed by non-analytical dependences of the NESS with respect to external variables. Phase diagram and criticalities are thus governed by the dependence of the low lying spectrum of the Liouvillian on these parameters. Despite the analogy with equilibrium QPTs, a comprehensive picture and characterisation of dissipative NESS-QPTs is lacking. This is partly due to their nature lying in a blurred domain, where features typical of zero temperature QPTs coexist with unexpected properties, some of which reminiscent of thermal phase transitions.

A natural approach to the investigation of such a novel scenario would be to adapt tools used in the equilibrium settings. We will illustrate how the use of the geometric methods, such as geometric phase [27,30], and in particular its mixed state generalisation, the Uhlmann geometric phase [156], may provide a novel perspective in the investigation of NESS-QPTs. Geometric phases and related geometrical tools, such as the Bures metrics [17,25,157], have been successfully applied in the analysis of many equilibrium phase transitions [42,158-161]. The Bures metrics have been employed in thermal phase transitions [162-164] and QPTs, both in symmetry-breaking [42,158-161,165,166] as well as in topological phase transitions [167].

Due to their mixed state nature, the NESSes require the use of a concept of geometric phase in the density operators domain. Among all possible approaches [156,168-174], the Uhlmann geometric phase [156] stands out for its deeprooted relation to information geometry and metrology [175,176], whose tools have been profitably employed in the investigation of QPTs and NESS-QPTs [159,166,177,178]. Uhlmann holonomy and geometric phase have been applied to the characterisation of both topological and symmetry breaking equilibrium QPTs [51,179-185]. Many proposals to measure the Uhlmann geometric phase have been put forward [186-188], and demonstrated experimentally [189].

Motivated by this, we will illustrate the role of the mixed state analogue of the Berry curvature, the Uhlmann curvature, in the characterisation of dissipative NESS-QPTs. The infinitesimal local properties of the manifold of density matrices can 
be captured by an object called mean Uhlmann curvature (MUC). The MUC is defined as the Uhlmann geometric phase per unit area of a density matrix evolving along an infinitesimal loop. It turns out that this quantity has also a fundamental interpretation in multi-parameter quantum metrology: it marks the incompatibility between independent parameters arising from the quantum nature of the underlying physical system [190]. In this sense, one can consider the MUC as a measure of "quantumness" in the multi-parameter estimation problem, and its singular behaviour responds only to quantum fluctuations occurring across a phase transitions. We will review these ideas, by showing paradigmatic examples of Fermionic quadratic dissipative Liouvillian models, some of which show rich NESS features $[123,124,177,178,191,192]$.

This review focuses on the role of the geometric phases in the investigation of equilibrium and non-equilibrium quantum phase transitions. However, we would not provide a fair account of the subject without touching upon the closely related subject of the fidelity approach [158,159,193-195], whose main achievements have been extensively reviewed in [160]. The original idea of the fidelity approach was to identify QPTs from the remarkable sensitivity of the Loschmidt echo [196] and the ground-state overlap [158,194] under the slightest changes of external parameters across criticality. This is a phenomenon which is strongly reminiscent of, and indeed related to, the well known Anderson orthogonality catastrophe [197]. Since its early development this approach has been applied successfully to a wide variety of systems, ranging from quasi-free Fermions [198,199], the Dicke model [158,161,193,200], matrix product state systems [201], BoseHubbard models [202,203], Stoner-Hubbard and BCS models [51]. Various methods have been developed to evaluate the fidelity, such as exact diagonalisation, density matrix renormalisation group [160,204,205], quantum Monte Carlo methods [195,206,207], tensor network algorithms [208,209]. This provides the means to venture into the study of models where analytical solutions are not available [160,204,205,207,210,211].

The fidelity approach has been employed in the investigation of first order phase transitions [212], in higherthan-second order QPTs [213], in finite temperature phase transitions [51,164,165,193,214] and non-equilibrium phase transitions [177,178,215-217]. The efficacy of this approach in signalling QPTs that elude the Landau-Ginzburg-Wilson paradigm has been tested against Berezinskii-Kosterlitz-Thouless phase transitions [218-222], topological phase transitions [167,223-225], and in strongly correlated models whose critical features are yet to be understood [210,211,226,227]. The universal scaling properties of quantities related to the fidelity approach, such as the fidelity susceptibility [195, 228,229], the quantum geometric tensor [42,160] and the Fisher information matrix [230], have been investigated and exploited in numerous scenarios.

Finally, one of the relevant features of these geometrical approaches is the potential experimental accessibility of many of their related quantities. Fidelity susceptibility [166,231,232], Fisher information [127,159], geometric phases [186-188] and geometric curvature $[118,119,233]$ can be probed through experimentally viable procedures.

To keep the review as pedagogical as possible, we will mostly focus the discussion on criticalities occurring in transverse field models, namely, Ising and XY models in a transverse magnetic field, which are paradigmatic examples exhibiting zero-temperature continuous QPTs. This will also pave the way to the second part of this review, where we will consider non-equilibrium versions of similar models. More than fifty years since their appearance, it is astounding how useful the transverse field models continue to be in the understanding of QPTs, non-equilibrium dynamics of quantum critical systems, and their connections to quantum information and geometric information.

\section{Continuous phase transition and universality}

We will consider only continuous QPTs, i.e. phase transitions associated to an order parameter that vanishes continuously at criticality, as opposed to first order QPT, characterised instead by abrupt changes in the order parameter. According to the standard classification, first order phase transitions are usually signalled by a finite discontinuity in the first derivative of the ground state energy density, whereas, continuous QPTs are identified by continuous first derivatives but discontinuous second derivatives of the ground state energy density.

Since many of the salient features of continuous phase transitions are common to classical and quantum phase transitions, let us take a brief detour into the topic of phase transitions driven by thermal fluctuations, and review some of the basic notions that will recur in the discussion on QPTs.

Assume that, at a given temperature $T=T_{c}$, a classical system with translation invariance symmetry in $d$-spatial dimensions has a critical point (CP). Any such critical point is characterised by several critical exponents, which identify most of the remarkable features of the system behaviour around the CP [16,234]. Let $O(\boldsymbol{r})$, with $\boldsymbol{r} \in \mathbb{T}^{d}$, denote an order parameter, i.e. a variable whose thermal expectation value $\langle O(\boldsymbol{r})\rangle=0$ for $T \geq T_{c}$ and $\langle O(\boldsymbol{r})\rangle \neq 0$ for $T<T_{c}$. If $T<T_{c}$, the two-point correlation function of the order parameter falls off exponentially at large distances, i.e. $\left\langle O\left(\boldsymbol{r}_{1}\right) O\left(\boldsymbol{r}_{2}\right)\right\rangle \sim$ $\exp \left(-\left|\boldsymbol{r}_{1}-\boldsymbol{r}_{2}\right| / \xi\right)$, where $\xi$ is the so called correlation length, which is a function of $T$. As the phase transitions are approached by varying some parameter, several remarkable phenomena occur. One of most notable is the divergence of $\xi$ as $T$ approaches $T_{c}$ from above, namely, as $T \rightarrow T_{c}^{+}$, usually as a power law which is characterised by the critical exponent, $v$, of correlation length, i.e. $\xi \sim\left(T-T_{c}\right)^{\nu}$. At criticality, i.e. at $T=T_{c}$, the divergence of the correlation length indicates that the two point correlation function decays only algebraically with the distance, namely $\left\langle O\left(\boldsymbol{r}_{1}\right) O\left(\boldsymbol{r}_{2}\right)\right\rangle \sim\left|\boldsymbol{r}_{1}-\boldsymbol{r}_{2}\right|^{-d-2+\eta}$, where $\eta$ is another critical exponent. Apart from the above long-ranged spacial correlation, similar scaling can be observed in the temporal behaviours at criticality. They are characterised by the dynamical critical exponent, $z$, which defines the scaling law of the system response-time $\tau_{c}=\omega_{c}^{-1}$ to external perturbations, which diverges like $\tau \sim \xi^{z}$ as $T \rightarrow T_{c}^{+}$, a phenomenon-known as critical slowing down. Correlation length $\xi$ and response-time $\tau_{c}$ set the only characteristic length 
scale and characteristic time scale close to the phase transitions. Infinite correlation length and time at criticality entails fluctuations on all length scales and timescales, and the system is said to be scale-invariant. A direct consequence is that every observable depends on the external parameters via power laws. The corresponding exponents are the critical exponents.

Apart from $z, v$ and $\eta$, other critical exponents are: $\beta$, which characterises the vanishing of the order parameter as the critical temperature is approached from below, i.e. $\langle O(\boldsymbol{r})\rangle \sim\left(T_{c}-T\right)^{\beta}$ as $T \rightarrow T_{c}^{-}$; $\alpha$ which defines the divergence of the singular part of the specific heat $C \sim\left(T-T_{c}\right)^{-\alpha}$ as $T \rightarrow T_{c}^{+}$. By denoting with $h$ the conjugate field to the order parameter, i.e. the field which couples to the $O(\boldsymbol{r})$ in the Hamiltonian of the system, one defines the zero-field susceptibility $\chi_{c}:=d\langle O(\boldsymbol{r})\rangle /\left.d h\right|_{h=0}$, whose critical exponent is $\gamma$. The latter characterises the divergence $\chi_{c} \sim\left(T-T_{c}\right)^{-\gamma}$ as $T \rightarrow T_{c}^{+}$. Exactly at $T=T_{c}$, the order parameter scales with $h$ as $O(\boldsymbol{r}) \sim|h|^{\frac{1}{\delta}}$ as $h \rightarrow 0$. The critical exponents, whose values characterise the type of criticality, are not independent of each other, many of them being related to one another through a set of scaling laws or exponent relations.

Moreover, it turns out that the complete set of critical exponents not only are mutually dependent, but they are the same across a whole class of phase transitions, called universal class. Indeed, one of the most astounding and far-reaching hallmark of continuous phase transitions is the notion of universality. Its most direct implication is that critical exponents are only determined by the symmetry of the order parameter, the dimensionality of the system and the nature of the fixed point, regardless of microscopic details of the Hamiltonian. The divergence of the correlation length and characteristic time are responsible for such a universal behaviour. Close to criticality, every physical quantity is averaged over all lengths that are smaller than the physically relevant scale set by the correlation length. This suggests that the universal critical behaviour can be satisfactorily described by an effective theory that keeps only the asymptotic long-wavelength degrees of freedom of the original Hamiltonian. On the other hand, specific properties which depends on the microscopic details of the model, e.g. the critical temperature, are not accessible through this coarse graining procedure, and are called nonuniversal properties. The paradigm of universality shifts the focus of the investigation from the specific model reproducing a peculiar critical phenomenon-occurring in nature to the study of an entire class of seemingly unrelated problems which are governed by the same universal features. Beyond its undoubted theoretical interest, this universality classification has an obvious practical advantage: relevant informations regarding a specific Hamiltonian model may be gleaned by exploring a simpler instance of the same universality class.

The above consideration on finite temperature phase transitions can be illustrated by turning to a specific example, the classical Ising model with ferromagnetic next-neighbour interaction, described by the Hamiltonian

$$
H=-J \sum_{\left\langle\boldsymbol{r} \boldsymbol{r}^{\prime}\right\rangle} \sigma_{\boldsymbol{r}}^{z} \sigma_{\boldsymbol{r}^{\prime}}^{z}-h \sum_{\boldsymbol{r}} \sigma_{\boldsymbol{r}}^{z}
$$

where $\sum_{\left\langle\boldsymbol{r} \boldsymbol{r}^{\prime}\right\rangle}$ denotes summation over next-neighbouring sites of a $d$-dimensional hyper-toric lattice, and $\sigma_{\boldsymbol{r}}^{z}$ is a Pauli $z$ matrix encoding a classical spin variable (assuming values \pm 1 ), which lies on the $\boldsymbol{r}$ th site of the lattice and which couples to a magnetic field of intensity $h$. For a vanishing magnetic field, the model shows a $\mathbb{Z}_{2}$ symmetry which may be spontaneously broken in a ferromagnetic phase with non-zero spontaneous magnetisation $m=1 / N\left\langle\sum_{\boldsymbol{r}} \sigma_{\boldsymbol{r}}^{z}\right\rangle_{h \rightarrow 0}$. The system undergoes a phase transitions as the critical temperature $T_{c}$ is crossed from below, from the ferromagnetic phase $\left(T \leq T_{c}\right)$ to a $\mathbb{Z}_{2}$ symmetric paramagnetic phase, with $m=0\left(T>T_{c}\right)$. In the special cases of $d \leq 2$, this model can be exactly solved with the transfer matrix method. For $d=1$, no spontaneous magnetisations are exhibited at any finite temperature $T>0$; whereas at $T=0$ the system shows long-range correlation, with a correlation length $\xi$ which diverges exponentially as $T \rightarrow T_{c}=0$. For $d \geq 2$, the classical Ising model exhibits positive critical temperatures $T_{c}>0$. One can appreciate in this example an instance of a general feature of classical critical models, i.e. the existence of a lower critical dimension, in this case $d_{c}^{l}=1$, which denotes the highest integer dimension for which a model displays a vanishing critical temperature. In the $d=2$ case, the model can be mapped to a one-dimensional transverse quantum Ising chain, which can be solved exactly, and provides a means of calculating all critical exponents. For example, the correlation length exponent is $v=1$, while the exponent associated to the spontaneous magnetisation $m=\left|T-T_{c}\right|^{\beta}$ is $\beta=1 / 8$.

\section{Quantum vs classical phase transitions}

Let us turn briefly to a fundamental question: to what extent a quantum mechanical formulation of a model is necessary in order to understand its critical phenomena, and to what extent will classical physics suffice? Are there distinctive features of a quantum critical phenomenon which cannot be understood in terms of a classical statistical model?

Within classical statistical mechanics, the statistical properties of a model are described in terms of its (canonical) partition function $Z:=\operatorname{Tr}\left[e^{-\beta H}\right]$, with $\beta:=1 / k_{B} T$ and $H=K+U$, which factorises into one term depending on the kinetic energy $K$ only and another that depends only on the potential energy $U$, i.e. $Z:=\operatorname{Tr}\left[e^{-\beta K}\right] \operatorname{Tr}\left[e^{-\beta U}\right]$. As a result, the static properties of the system can be studied independently of the dynamical ones. Moreover, the kinetic energy, which is generically expressed as $K:=\sum_{j} p_{j}^{2} /(2 m)$ in terms of the generalised momenta $p_{i}$, contributes to the partition function $Z$ with a Gaussian factor $e^{-\beta K}$, which is analytic. Hence, any singular behaviour of $Z$ which results in a phase transitions can only arise from the potential energy factor. In particular, the dynamical exponent $z$ is independent of all of the other critical exponents, and the static critical behaviour can be studied by means of an effective functional of a time-independent order parameter. By contrast, in quantum systems the situation differs quite fundamentally, as in 
general $[K, U] \neq 0$. One then sees that the statics and the dynamics of quantum systems are intrinsically coupled and need to be treated together and simultaneously. As a consequence, the dynamical exponent $z$ becomes an integral part of the set of exponents of a given universality class.

A way of telling whether quantum mechanics is important in the description of a critical phenomenon is comparing the thermal fluctuations of the order parameter and its quantum fluctuations, which are set by the smallest energy scale of the relevant quantum degrees of freedom. A rule of thumb is contrasting the two most significant energy scales, namely $\hbar \omega_{c}$, the energy of long-distance order parameter fluctuations, and the thermal energy, $k_{B} T$. In practise, one says that the order parameter fluctuations change their character from quantum to classical when $\hbar \omega_{c}$ falls below $k_{B} T$. However, for any thermal phase transitions, the typical energy scale vanishes as $\hbar \omega_{c} \sim \xi^{z} \sim\left|T-T_{c}\right|^{z \nu}$. Therefore quantum mechanics becomes necessarily unimportant, and the critical behaviour at the transitions is dominated by classical fluctuations. This explains the name "classical transitions" for transitions occurring at finite temperature.

\section{Quantum phase transitions}

The situation is different for transitions occurring at $T=0$, driven by a set of non-thermal control parameters $\lambda=\left\{\lambda_{1} \ldots \lambda_{N}\right\}$, like pressure or magnetic field. In this case the fluctuations in the order parameter are dominated by quantum mechanics, which therefore justifies calling this type of criticality "quantum" phase transitions [7].

Quantum phase transitions are therefore criticalities arising for $T=0$, where the system lies in its Hamiltonian ground state. This is generally uniquely determined by the values of the parameters $\lambda$ of its Hamiltonian $H(\lambda)$, unless something "singular" happens in the spectrum of the many-body system for some critical values $\lambda_{c}$. Such a non-analyticity may be due to a simple level crossing in the many-body ground state. This possibility can only arise when a $\lambda$ couples to a conserved quantity of the full Hamiltonian, i.e. $H(\lambda)=H_{0}+\lambda H_{1}$, with $\left[H_{0}, H_{1}\right]=0$. This means that, while the eigenvalues will change as a function of the Hamiltonian parameters, the eigenstates will be independent of $\lambda$. Hence, a level-crossing may well occur, creating a point of non-analyticity of the ground state energy, however, it does not determine critical singularities in the correlations, and it gives rise to a first-order quantum phase transitions. This is a type of transitions that also finite-size systems can exhibit.

A totally different story is what happens for continuous phase transitions, which are characterised by higher-order singularities in the energy density. This occurs when a system ground state energy, whose finite-size spectrum displays an avoided level crossing, reaches an infinitely sharp transitions in the thermodynamic limit. This involves infinitely many eigenstates of the many-body system, and the thermodynamic limit is needed for such a singularity to arise. In this case, it is the non-commutativity of the Hamiltonian terms which is responsible for the quantum fluctuations which drive the systems across the quantum phase transitions. One might think that phase transitions occurring at zero temperature are not physically relevant to the actual world. However, one can show that many finite temperature features of a system can be gleaned through the properties of its quantum critical point.

From the above considerations, the point of singularity in the ground state energy density is associated with an energy scale $\Delta$ which vanishes as $\lambda$ approaches a critical value $\lambda_{c}$. This energy scale is generally identified by the energy difference between the ground state and the first excited state, i.e. the energy gap, and its dependence on the system parameter is generally algebraic in the proximity of the criticality i.e.

$$
\Delta \sim J\left|\lambda-\lambda_{c}\right|^{\nu z} .
$$

Here $J$ is an energy scale associated to the microscopic details of system couplings, and $z$ and $v$ are critical exponents characteristic of the critical point $\lambda_{c}$. Similarly to continuous thermal phase transitions, a QPTs may be characterised by an order parameter $O(\boldsymbol{r}, t)$, which is an observable whose expectation value vanishes continuously, as a function of $\lambda$, across the critical point $\lambda_{c}$, going from one phase (the ordered phase) to the other (the disordered phase). One can define a length scale $\xi$ which typically characterises the exponential decay of the equal-time two-point correlation function of the ground state,

$$
G\left(\boldsymbol{r}-\boldsymbol{r}^{\prime}\right):=\left\langle O(\boldsymbol{r}, t) O\left(\boldsymbol{r}^{\prime}, t\right)\right\rangle-\langle O(\boldsymbol{r}, t)\rangle\left\langle O\left(\boldsymbol{r}^{\prime}, t\right)\right\rangle \sim \frac{e^{-\left|\boldsymbol{r}-\boldsymbol{r}^{\prime}\right| / \xi}}{\left|\boldsymbol{r}-\boldsymbol{r}^{\prime}\right|^{d-2+\eta}} .
$$

Here, $\eta$ is another critical exponent, characterising the power-law decay of correlations $G(\boldsymbol{r}) \sim|\boldsymbol{r}|^{-d-2+\eta}$ at exactly $\lambda=\lambda_{c}$. In quantum continuous phase transition one invariably observes the algebraic divergence of the correlation length approaching the critical point

$$
\xi \sim\left|\lambda-\lambda_{c}\right|^{-v} \text {. }
$$

Similarly, one can define a time scale $\tau_{c}$ for the decay of equal-space correlations at quantum phase transitions

$$
\tau_{c} \sim \Delta^{-1} \propto \xi^{z} \propto\left|\lambda-\lambda_{c}\right|^{-v z}
$$




\section{Geometric phase and criticality}

A characteristic that all non-trivial geometric evolutions have in common is the presence of non-analytic points in the energy spectrum. At these points, the state of the system is not well defined owing to their degenerate nature. One could say that the generation of a geometric phase (GP) is a witness of such singular points. Indeed, the presence of degeneracy at some point is accompanied by curvature in its immediate neighbourhood, and a state that evolves along a closed path is able to detect it. These points or regions are of great interest to condensed matter or molecular physics as they determine, to a large degree, the behaviour of complex quantum systems. The geometric phases are already used in molecular physics to probe the presence of degeneracy in the electronic spectrum of complex molecules. Initial considerations by Herzberg \& Longuet-Higgins [235] revealed a sign reversal when a real Hamiltonian is continuously transported around a degenerate point. Its generalisation to the complex case was derived by Stone [236] and an optimisation of the real Hamiltonian case was performed by Johansson \& Sjöqvist [237,238].

Geometric phases have been associated with a variety of condensed matter and solid-state phenomena. They are at the core of the characterisation of topological phase transitions [34-36], and have been employed in the description and detection of QPT, both theoretically [31,32,37-40,76] and experimentally [41]. However, their connection to quantum phase transitions has been put forward only in the last decade [31,37]. It was further elaborated by Zhu [39], where the critical exponents were evaluated from the scaling behaviour of geometric phases, and by Hamma [32], who showed that geometric phases can be used as a topological test to reveal quantum phase transitions. The use of GP in QPTs can be heuristically understood as follows. As we have seen, quantum phase transitions are associated by dramatic structural changes of the system state, resulting from small variations of control parameters. These critical changes are accompanied by the presence of degeneracies in the ground state energy density of the many-body system. The degeneracy are at the origin of the non-analyticity of the ground-state wave function, which characterises the long-range quantum correlations at criticality $[31,32,37-40,76]$.

In this section we explore the ability of geometric phase and related quantities to reveal quantum critical phenomena in many-body quantum systems. The use of geometric phases provides a new conceptual framework to understand quantum phase transitions, and at the same time suggests novel viable approaches to experimentally probe criticalities. This maybe done through the evolution of the quantum many-body system in the neighbourhood of a critical point, in a way that does not take the system directly through a quantum phase transitions. The latter is hard to physically implement as it is accompanied by multiple degeneracies that can take the system away from its ground state.

Moreover, the geometric phase approach is not based on the identification of an order parameter - and therefore does not require a knowledge of symmetry breaking patterns - or more in general on the analysis of any distinguished observable, e.g., Hamiltonian, but it is a purely geometrical characterisation.

This geometric phase approach has been applied in quite a few explicit models $[31,38,40,76]$. However, for the sake of simplicity we will discuss two of them which stand out for their richness and at the same time for their simplicity, i.e. the paradigmatic one dimensional Ising, and XY models in transfer magnetic field [31,37] and the Dicke model [38], whose geometric phase properties will be briefly discussed in the next few sections.

The one-dimensional version of the transverse Ising model emerged for the first time in the resolution of the two-dimensional nearest-neighbour ferromagnetic classical Ising model. The row-to-row transfer matrix of the twodimensional classical model converges to the transverse Ising chain in a suitable limit [239], and its exact solution soon follows [240]. This correspondence represents the prototypical example of a quantum-to-classical mapping. The model was employed, shortly later, to reproduce the ferroelectric transitions in Potassium Dihydrogen Phosphate [241].

The Ising model and its generalisation, the XY model, are analytically solvable and they offer enough control parameters to support geometric evolutions. Moreover, in its rich criticality structure the XY model includes the XX critical model. By explicit calculations one can observe that an excitation of the model gains a non-trivial geometric phase if and only if it circulates a region of criticality, a feature which expresses the topological origin of the geometric phase. The generation of this phase can be traced down to the presence of a conical intersection of the energy levels located at the XX criticality in an equivalent way used in molecular systems. The scaling of the geometric phase can be used to obtain the critical exponents that completely characterise the critical behaviour. It is not hard to generalise these results to the case of an arbitrary spin system, which sheds light on the understanding of more general systems, where analytic solutions might not be available.

\subsection{Berry's phase}

Historically, the definition of geometric phase was originally introduced by Berry in a context of closed, adiabatic, Schrödinger evolution. What Berry showed in his seminal paper [27] was that a quantum system subjected to a slowly varying Hamiltonian manifests in its phase a geometric behaviour due to the structure of the Hilbert space. When the Hamiltonian of a system evolves cyclically and slowly enough, any (non-degenerate) eigenstate of the initial Hamiltonian evolves adiabatically following the instantaneous eigenspace. When the Hamiltonian returns to its original value, the system is eventually brought back to the ray of its initial state, acquiring a phase that, apart from the usual dynamical phase, is geometrical in nature. This phase is called Berry's phase. 
Let us summarise the derivation of the Berry phase and show how this geometric property comes naturally from the solution of the Schrödinger equation. Consider a Hamiltonian $H(\lambda)$ depending on some external parameters $\lambda=$ $\left(\lambda_{1}, \lambda_{2}, \ldots, \lambda_{n}\right)$, and suppose that these parameters can be varied arbitrarily inside a space $\mathcal{M}$ (the parameter space). Assume that for each value of $\lambda$ the Hamiltonian has a completely discrete spectrum of eigenvalues, given by the equation

$$
H(\lambda)|n(\lambda)\rangle=\epsilon_{n}(\lambda)|n(\lambda)\rangle,
$$

where $|n(\lambda)\rangle$ and $\epsilon_{n}(\lambda)$ are smooth concatenations of eigenstates and eigenvalues, respectively, of $H(\lambda)$, as functions of the parameters $\lambda$. Suppose that the values of the parameters evolve smoothly along a curve $\lambda(\tau) \in \mathcal{M}(\tau \in[a, b])$, respecting the prescription of the adiabatic theorem, i.e that the rate at which the parameters evolve is low compared to the time scales of the Bohr frequencies of the system $\left(\epsilon_{n}(\lambda)-\epsilon_{m}(\lambda)\right.$ ) (as usual assume $\hbar=1$ ). The adiabatic theorem states that, under such a regime, an eigenstate of the system evolves following "rigidly" the transformation of the Hamiltonian: a system, initially prepared in an eigenstate with eigenvalue $\epsilon_{n}(\lambda(a))$, remains at any instant $t$ of its evolution in the eigenspace $\epsilon_{n}(\lambda(t))$, i.e. the eigenspace smoothly connected with the initial eigenspace $\epsilon_{n}(\lambda(a))$.

Thus, in the simplest case of non-degenerate eigenvalues, since the eigenspace is one-dimensional, the evolution of any eigenstate is specified by the spectral decomposition (6) up to a phase factor. The adiabatic approximation introduces a constraint only on the direction of the vector state at any instant of time, and the eigenvalue equation (6) implies no relation between the phases of the eigenstates $|n(\lambda)\rangle$ at different $\lambda$ 's. Thus, for the present purpose any (smooth) choice of phases can be made. Then, a state $\left|\psi_{n}\right\rangle$ initially in the eigenstate $|n(\lambda(a))\rangle$, evolves as

$$
\left|\psi(t)_{n}\right\rangle \simeq \exp \left\{-i \int_{a}^{t} \epsilon_{n}(\lambda(\tau)) d \tau\right\} \exp i \phi_{n}^{B}(t)|n(\lambda(t))\rangle,
$$

where the first phase factor is the usual dynamic one, and the second one is an additional phase that is introduced to solve the dynamics of the system.

The novel idea introduced by Berry was to recognise that this additional phase factor has an inherent geometrical meaning. The crucial point is that this phase $\phi_{n}^{B}$ is non-integrable, i.e. it cannot be written as a single valued function of $\lambda$. Its actual value must be determined as a function of the path followed by the state during its evolution. The most important thing is that this value depends only on the geometry of this path, and not on the rate at which it is traversed. Or, in a more formal way, it is independent of the parameterisation of the path.

Under the assumption of the adiabatic approximation, this phase can be determined by requiring that $\left|\psi(t)_{n}\right\rangle$ satisfies the solution of the Schrödinger equation. A direct substitution of the expression (7) into

$$
H(\lambda(t))\left|\psi(t)_{n}\right\rangle=i \frac{d}{d t}\left|\psi(t)_{n}\right\rangle,
$$

leads to

$$
\frac{d \phi_{n}^{B}(t)}{d t}=i\left\langle n(\lambda(t))\left|\frac{d}{d t}\right| n(\lambda(t))\right\rangle .
$$

Therefore $\phi_{n}^{B}(t)$ can be represented by a path integral in the parameter space $\mathcal{M}$,

$$
\phi_{n}^{B}(t)=i \int_{a}^{b}\left\langle n(\lambda(t))\left|\frac{d}{d t}\right| n(\lambda(t))\right\rangle d t=\int_{\lambda(a)}^{\lambda(b)} \boldsymbol{A}^{B},
$$

where $\boldsymbol{A}^{B}:=\sum_{\mu} A_{\mu}^{B} d \lambda_{\mu}$ is called Berry connection, a differential one-form, whose elements, with respect to the local coordinates $\left\{\lambda_{\mu}\right\}$, are defined as

$$
A_{\mu}^{B}=i\left\langle n(\lambda)\left|\partial_{\mu}\right| n(\lambda)\right\rangle,
$$

where $\partial_{\mu}:=\partial / \partial \lambda_{\mu}$. This phase $\phi_{n}^{B}$ becomes physically relevant and non-trivial only when the parameters are changed along a closed path, such that $\lambda(a)=\lambda(b)$. Otherwise, the geometrical phase can be factored out by choosing a suitable eigenbasis. This non-trivial phase, the Berry phase, is then given by

$$
\phi_{n}^{B}(C)=\oint_{C} \boldsymbol{A}^{B} .
$$

This is nothing but a line integral of a vector potential, (analogous to the electromagnetical vector potential) around a closed path in the parameter space. As this quantity is not identically zero, it implies that the phase acquired is not integrable in nature. It is not possible to define the phase as a single valued function of the parameter space, because the phase depends on the previous history of the state, i.e. on the path that it has followed to arrive to this point. By exploiting the Stokes theorem in the n-dimensional space $\mathcal{M}$, this quantity can be written as an integral on the (oriented) surface $\Sigma(C)$ bounded by the closed curve $C$

$$
\phi_{n}^{B}(C)=\int_{\Sigma(C)} \boldsymbol{F}^{B}
$$


where $\boldsymbol{F}^{B}:=d \boldsymbol{A}^{B}=\frac{1}{2} \sum_{\mu \nu} F_{\mu \nu}^{B} d \lambda_{\mu} \wedge d \lambda_{\nu}$ is the Berry curvature (differential two-form), where $d \lambda_{\mu} \wedge d \lambda_{\nu}$ is the infinitesimal surface element of $\Sigma(C)$, spanned by the two independent directions $\lambda_{\mu}$ and $\lambda_{\nu}$ of $\mathcal{M}$, and $\left[F_{\mu \nu}^{B}\right]$ is an antisymmetric tensor field (analogous to the electromagnetic tensor field), with components

$$
F_{\mu \nu}^{B}:=\partial_{\mu} A_{\nu}-\partial_{\nu} A_{\mu}=\left\langle\partial_{\mu} n(\lambda) \mid \partial_{\nu} n(\lambda)\right\rangle-\left\langle\partial_{\nu} n(\lambda) \mid \partial_{\mu} n(\lambda)\right\rangle,
$$

or, in a coordinate independent way,

$$
\boldsymbol{F}^{B}=\langle\operatorname{dn}(\lambda)|\wedge| \operatorname{dn}(\boldsymbol{\lambda})\rangle,
$$

where $|d n\rangle:=d|n\rangle .{ }^{1}$ As already pointed out, $|n(\lambda)\rangle$ is only one choice out of infinitely many possible concatenation of states that satisfy the eigenvalue Eq. (6). If $|n(\lambda)\rangle$ is a solution of Eq. (6), $e^{i \alpha(\lambda)}|n(\lambda)\rangle$ also satisfies the same eigenvalue problem. The freedom of choice of a particular $|n(\lambda)\rangle$ is often referred to a gauge freedom, in analogy to the $U(1)$ gauge freedom of the electromagnetic vector potential. From the definition (11) it follows that the Berry connection $A$ does depend on the gauge choice; indeed it transforms according to

$$
\begin{aligned}
|n(\lambda)\rangle & \rightarrow\left|n^{\prime}(\lambda)\right\rangle=e^{-i \alpha(\lambda)}|n(\lambda)\rangle \\
A_{\mu}^{B} & \rightarrow A^{B^{\prime}}{ }_{\mu}=A_{\mu}^{B}+\partial_{\mu} \alpha(\lambda)
\end{aligned}
$$

Notice that such a phase transformation reveals a gauge structure of the Berry connection analogous to the one of an electromagnetic vector potential. By following the electromagnetic analogy, we can expect that, although $A$ is gauge dependent, the tensor field $F_{\mu \nu}^{B}$ should be invariant under this transformations. It is easily verified that under gauge change, one gets

$$
F_{\mu \nu}^{B} \rightarrow F_{\mu \nu}^{B^{\prime}}=\partial_{\mu} A^{B^{\prime}}{ }_{\nu}-\partial_{\nu} A^{B^{\prime}}{ }_{\nu}=\partial_{\nu} A_{\mu}-\partial_{\mu \nu}^{2} \alpha-\partial_{\nu} A_{\mu}^{B}+\partial_{\nu \mu}^{2} \alpha=F_{\mu \nu}^{B},
$$

which consequently demonstrates the gauge independence of $\phi_{n}^{B}(C)$ itself. This reinforces the idea that $\phi_{n}^{B}(C)$ is indeed a physical observable effect, independent of unessential phase choices. Moreover, the expression of $\phi_{n}^{B}(C)$ as a path integral in the parameter space guarantees that it does not depend on the rate of traversal of the circuit $C$, provided the adiabatic approximation holds. Therefore, the Berry phase, a natural consequence of the Schrödinger evolution and the adiabatic approximation, respects the essential requirements to be a geometric feature: (i) gauge independence, (ii) parameterisation invariance. As we already mentioned, the eigenvalue equation (6) implies no relation between the phases of the instantaneous eigenstates $|n(\lambda)\rangle$ at different $\lambda$. It is Eq. (9) which imposes constraints on the phase acquired by the time dependent eigenstates. By subtracting the dynamical phase, this constraints can be rephrased in a compact form. By absorbing the phase factor into the definition of the eigenstates as follows

$$
|\tilde{n}(t)\rangle=e^{i \phi^{B}(t)}|n(\lambda(t))\rangle,
$$

Eq. (9) becomes a condition on the possible eigenstates $\tilde{n}$ satisfying the time evolution along $\phi^{B}(t)$

$$
\left\langle\tilde{n}(t)\left|\frac{d}{d t}\right| \tilde{n}(t)\right\rangle=0 .
$$

This constraint is the parallel transport condition, which literally requires the time derivative of the instantaneous eigenvector to have vanishing component along the direction of the eigenvector itself. The term parallel transport is to be understood in the sense that neighbouring states along the curve are chosen "as parallel as possible". This quantitatively means that Eq. (18) maximises the scalar product between infinitesimally closed states

$$
|\langle\tilde{n}(t) \mid \tilde{n}(t+d t)\rangle|^{2} \simeq 1-2\left|\left\langle\tilde{n}(t)\left|\frac{d}{d t}\right| \tilde{n}(t)\right\rangle\right| d t .
$$

Solving Eq. (18) amounts to choosing a particular smooth concatenation of eigenstates $|\tilde{n}(t)\rangle$ with a special property: each state and its neighbouring are in phase, i.e. $\arg \langle\tilde{n}(t) \mid \tilde{n}(t+d t)\rangle \simeq 0$. Although, locally the states are in phase, a global phase accumulates as the path is traversed. If compared, the two endpoints of this chain reveal a relative phase which is the Berry's phase

$$
\langle\tilde{n}(T) \mid \tilde{n}(0)\rangle=e^{i \phi^{B}(C)} .
$$

\footnotetext{
1 Throughout this review we will sometimes make use of " $d$ " in the sense of exterior derivative. In differential geometry, the exterior derivative is the generalisation of the concept of differential applied to $k$-forms. For a scalar $f$ (a differential 0 -form), the application of the exterior derivative yields the usual differential of calculus, i.e. $d f=\sum_{\mu} \partial_{\mu} f d \lambda_{\mu}$. In general, the exterior derivative is the unique mapping from $k$-forms to $(k+1)$-forms, satisfying the following properties:

1. $d f$ is the differential of $f$ for smooth functions $f$;

2. $d(d f)=0$, (or in short $d^{2}=0$ ), for any (smooth) form $f$;

3. $d(f \wedge g)=d f \wedge g+(-1)^{k} f \wedge d g$, where $f$ is a $k$-form.

This leads to a generalised Stokes' theorem (or the generalised fundamental theorem of calculus), in the following form; if $\mathcal{M}$ is a compact smooth orientable $\mathrm{n}$-dimensional manifold with boundary $\partial \mathcal{M}$, and $\omega$ is an $(n-1)$-form on $\mathcal{M}$, then [26]
}

$$
\int_{\mathcal{M}} d \omega=\int_{\partial \mathcal{M}} \omega
$$




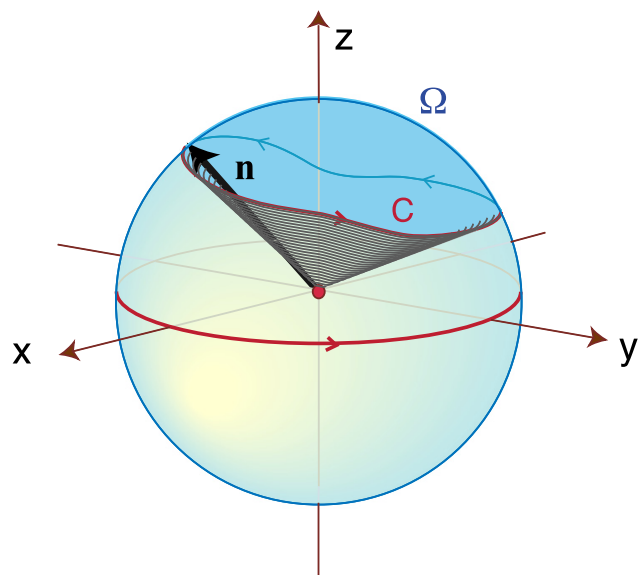

Fig. 1. The geometric phase is proportional to the solid angle spanned by the Hamiltonian with respect of its degeneracy point.

This is the original result of Berry: the state of the system, after a closed adiabatic evolution, returns to a state $\tilde{n}(T)$ that gains an irreducible part in its phase $\phi^{B}(\mathcal{C})$, in addition to the dynamical contribution. This phase, analogously to the definition of phase that we have shown in the previous section, has an inherent geometrical meaning, since it does not depend on either the detail of time evolution or unessential phase transformations.

\subsection{A simple two-level system}

Before going into the details of the geometric phase for a many-body system, it pays to have a brief detour to the simplest, yet significant example of geometric phase, i.e. the one arising in a two-level system. As in the previous subsection, we will deal with geometric phases arising from the adiabatic evolution of the eigenstate of a Hamiltonian. Consider a $2 \times 2$ Hamiltonian $H(\lambda)$, where $\lambda$ is a set of parameters. It can always be expressed, up to an irrelevant identity matrix, as

$$
H(\lambda)=\mathbf{n}(\lambda) \cdot \boldsymbol{\sigma}=|\mathbf{n}| g(\theta, \varphi) \sigma^{z} g^{\dagger}(\theta, \varphi),
$$

where $\tan \theta:=\sqrt{n_{x}^{2}+n_{y}^{2}} / n_{z}$ and $\tan \varphi:=n_{y} / n_{x}$. Here

$$
g=e^{-i \sigma^{z} \varphi / 2} e^{-i \sigma^{y} \theta / 2}
$$

is a $S U(2)$ transformation which rotates the operator $\mathbf{n} \cdot \vec{\sigma}$ to the $z$-direction, and the vector, $\sigma:=\left(\sigma^{x}, \sigma^{y}, \sigma^{z}\right)$, of Pauli's operators is given by

$$
\sigma^{x}=\left(\begin{array}{ll}
0 & 1 \\
1 & 0
\end{array}\right), \quad \sigma^{y}=\left(\begin{array}{cc}
0 & -i \\
i & 0
\end{array}\right), \quad \sigma^{z}=\left(\begin{array}{cc}
1 & 0 \\
0 & -1
\end{array}\right) .
$$

Using this parameterisation one can represent the Hamiltonian as a tridimensional vector on a sphere, centred in the point of degeneracy of the Hamiltonian $(|\mathbf{n}|=0)$, (see Fig. 1).

For $\theta=\varphi=0$ we have that $g=\mathbb{1}$ and the two eigenstates of the system are given by $|+\rangle=(1,0)^{T}$ and $|-\rangle=(0,1)^{T}$ with corresponding eigenvalues $E_{ \pm}= \pm|\mathbf{n}| / 2$. Let us consider the evolution resulting when a closed path $C$ is spanned adiabatically on the sphere. Following the general considerations it is easy to show that the only non-zero component of the Berry connection, $\mathbf{A}^{B}$, is given by

$$
A_{\varphi}^{B}= \pm \frac{1}{2}(1-\cos \theta)
$$

that leads to the Berry phase

$$
\phi^{B}(C)=\oint_{C} \mathbf{A}^{B} \cdot d r=\frac{1}{2} \int_{\Sigma(\theta, \varphi)} \sin \theta d \theta d \varphi=\frac{\Omega}{2} .
$$

Here $\Omega=\iint_{\Sigma} \sin \theta d \theta d \varphi$ is the solid angle enclosed by the loop, as seen from the degeneracy point. In this expression the geometric origin of the Berry phase is evident. Its value depends only on the path followed by the parameters and not on the detail of the evolution, and indeed it depends only on the way in which these parameters are changed in relation with the degeneracy point of the Hamiltonian.

A particularly interesting case is the one in which the Hamiltonian can be casted in a real form, corresponding in this case to $\theta=\pi / 2$. In this case the phase (23) becomes $\pi$ reproducing the sign change of the eigenstate, as this is circulated around a point of degeneracy $(|\mathbf{n}|=0)$, in agreement with the Longuet-Higgins theorem. 


\subsection{The $X Y$ model and its criticalities}

The first model that we will employ to illustrate the connection between geometric phases and critical systems is the spin-1/2 chain with XY interactions. This is a one-dimensional model where the spins interact with their nearest neighbours via the Hamiltonian

$$
H=-\sum_{j=1}^{n}\left(\frac{1+\delta}{2} \sigma_{j}^{x} \sigma_{j+1}^{x}+\frac{1-\delta}{2} \sigma_{j}^{y} \sigma_{j+1}^{y}+\frac{h}{2} \sigma_{j}^{z}\right),
$$

where $n$ is the number of spins, $\sigma_{j}^{\mu}$ are the Pauli matrices at site $j, \delta$ is the $\mathrm{x}-\mathrm{y}$ anisotropy parameter and $h$ is the strength of the magnetic field. This model was first solved explicitly by Lieb, Schultz and Mattis [239] and by Katsura [240]. Since the XY model is exactly solvable and still presents a rich structure it offers a benchmark to test the properties of geometric phases in the proximity of criticalities.

In particular, we are interested in a generalisation of Hamiltonian (91) obtained by applying to each spin a rotation of $\varphi$ around the $z$-direction

$$
H(\varphi)=g(\varphi) H g^{\dagger}(\varphi) \quad \text { with } \quad g(\varphi)=\prod_{j=1}^{n} e^{i \sigma_{j}^{z} \varphi},
$$

in the same way as we did in the case of a single spin-1/2. The family of Hamiltonians that is parameterised by $\varphi$ is clearly isospectral and, therefore, the critical behaviour is independent of $\varphi$. This is reflected in the symmetric structure of the regions of criticality shown in Fig. 2. In addition, due to the bilinear form of the interaction terms we have that $H(\varphi)$ is $\pi$-periodic in $\varphi$. The Hamiltonian $H(\varphi)$ can be diagonalised by a standard procedure [31], which can be summarised in the following three steps:

- the Jordan-Wigner transformation, which converts spin operators into Fermionic operators via the relations,

$$
c_{l}:=\left(\prod_{m<l} \sigma_{m}^{z}\right)\left(\sigma_{l}^{x}+i \sigma_{l}^{y}\right) / 2, \quad\left\{c_{j}, c_{l}^{\dagger}\right\}=\delta_{j l}, \quad\left\{c_{j}, c_{l}\right\}=0 ;
$$

- a Fourier transform,

$$
d_{k}=\frac{1}{\sqrt{n}} \sum_{l} c_{l} e^{-i 2 \pi l k / n}, \text { with } k=-0, \ldots, n-1,
$$

with, $\quad\left\{d_{k}, d_{k^{\prime}}^{\dagger}\right\}=\delta_{k k^{\prime}},\left\{d_{k}, d_{k^{\prime}}\right\}=0$;

- a Bogoliubov transformation, which defines the Fermionic operators,

$$
b_{k}=d_{k} \cos \frac{\theta_{k}}{2}-i d_{-k}^{\dagger} e^{i \phi} \sin \frac{\theta_{k}}{2},
$$

where the angle $\theta_{k}$ is defined as $\theta_{k}:=\arccos \left(\eta_{k} / \varepsilon_{k}\right)$ with $\eta_{k}:=\cos \frac{2 \pi k}{n}-h$ and

$$
\varepsilon_{k}:=\sqrt{\eta_{k}^{2}+\delta^{2} \sin ^{2} \frac{2 \pi k}{n}}
$$

is the energy of the single eigenmode $d_{k}$ of pseudo-momentum $k$, called energy dispersion relation. These procedures diagonalise the Hamiltonian to the form

$$
H(\varphi)=\sum_{k=0}^{M} \varepsilon_{k} b_{k}^{\dagger} b_{k} .
$$

where either $M=n / 2-1$, if $n$ is even, or $M=(n-1) / 2$, if $n$ is odd. The ground state $|g\rangle$ of $H(\varphi)$ is the vacuum of the Fermionic modes, $b_{k}$, given by

$$
|g\rangle:=\bigotimes_{k}\left(\cos \frac{\theta_{k}}{2}|0\rangle_{k}|0\rangle_{-k}-i e^{i \varphi} \sin \frac{\theta_{k}}{2}|1\rangle_{k}|1\rangle_{-k}\right),
$$

where $|0\rangle_{k}$ and $|1\rangle_{k}$ are the vacuum and single excitation of the $k$ th mode, $d_{k}$, respectively. The energy gap is clearly given by the minimum of the energy dispersion relation $\varepsilon_{k}$. From (30) one can interpret the ground state as the direct product of $n$ spins, each one oriented along the direction $\left(\varphi, \theta_{k}\right)$. The critical points in the XY model are determined by the conditions under which the ground and the first excited states become degenerate, which in this case amounts to a vanishing energy dispersion relation $\varepsilon_{k}$. This is the only condition in which singularities may arise. There are two distinct regions of the space diagram that are critical. When $\delta=0$, we have $\varepsilon_{k}=0$ for $-1 \leq h \leq 1$, which is a first-order phase transitions with an actual energy crossing and critical exponents $z=2$ and $v=1 / 2$. The other 

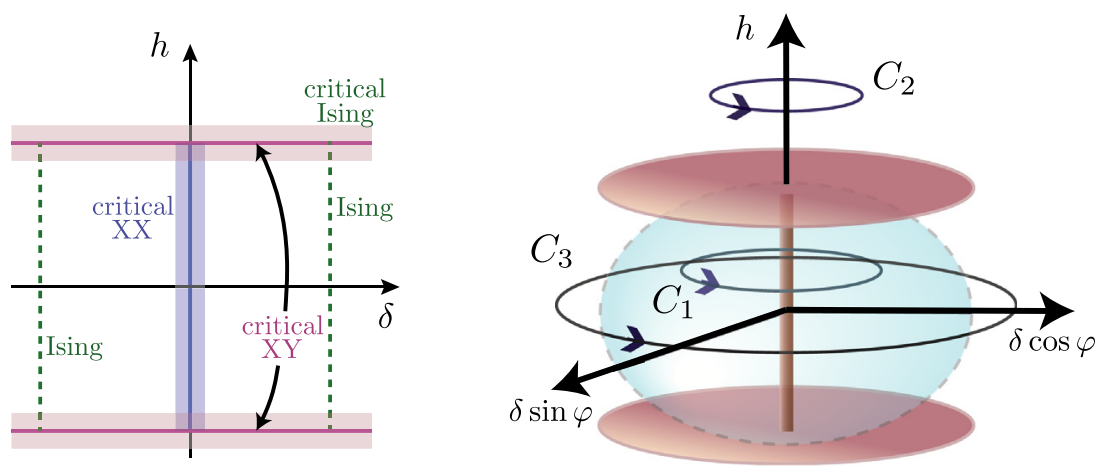

Fig. 2. On the left, the regions of criticality of the $H$ Hamiltonian are presented as a function of the parameters $h$ and $\delta$. The corresponding regions of criticality for the Hamiltonian $H(\varphi)$ are shown on the right, where $\varphi$ parameterises a rotation around the $h$ axis. Possible paths for the geometrical evolutions are depicted, spanned by varying the parameter $\varphi$.

(a)

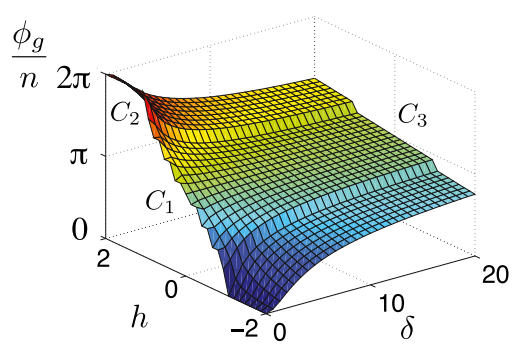

(b)

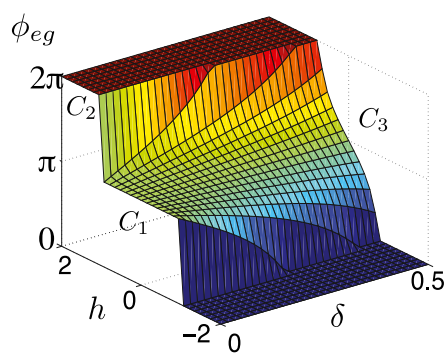

Fig. 3. The geometric phase corresponding to the ground state (a) and the relative one between the ground and excited state (c) as a function of the path parameters $h$ and $\delta$. Each point of the surface corresponds to the geometrical phase for a path that is spanned by varying $\varphi$ from 0 to $\pi$ for certain $h$ and $\delta$. The values of the geometric phase corresponding to the loops $C_{1}, C_{2}$ and $C_{3}$ in Fig. 2 are also indicated.

critical region is given by $h= \pm 1$ where one finds $\varepsilon_{k}=0$ for all $\delta$. These are continuous phase transitions, with finite-size ground-state spectrum having avoiding-crossing. When $\delta=1$ and $h= \pm 1$, we obtain the Ising critical model with critical exponents $z=1$ and $v=1$. Finally, let us consider the criticality behaviour of the rotated $H(\varphi)$. Clearly, the energy dispersion relation $\varepsilon_{k}$ does not depend on the angle $\varphi$, as this parameter is related to an isospectral transformation. Hence, the criticality region for the rotated Hamiltonian is obtained just by a rotation around the $h$ axis. This is illustrated in Fig. 2, where the Ising-type criticality corresponds now to two planes at $h=1$ and $h=-1$ and the XX criticality is along the $h$ axis for $|h|<1$.

\subsection{Geometric phases and $X Y$ criticalities}

Fig. 2 depicts the critical points of the XY model. Now, we are interested in obtaining looping trajectories in the parameter space described by the Hamiltonian variables $h, \delta$ and $\varphi$. The aim is to determine the geometric evolutions corresponding to these paths and relate them to regions of criticality. An especially interesting family of closed paths is the one in which loops circulate around the $h$ axis, as the parameter $\varphi$ varies from zero to $\pi$. Indeed, these paths may enclose the XX criticality [7], depending on whether $-1<h<1$. It is possible to evaluate the corresponding geometric phases of the ground and the first excited states as a function of $h$ and $\delta$.

Using the standard formula (10), it is easy to show that the Berry phase of the ground state $|g\rangle$ is given by

$$
\phi_{g}=-i \int_{0}^{2 \pi}\left\langle g\left|\frac{\partial}{\partial \varphi}\right| g\right\rangle d \varphi=\sum_{k>0} \pi\left(1-\cos \theta_{k}\right) .
$$

This result can be understood by considering the form of $|g\rangle$, which is a tensor product of states, each lying in the two dimensional Hilbert space spanned by $|0\rangle_{k}|0\rangle_{-k}$ and $|1\rangle_{k}|1\rangle_{-k}$. For each value of $k(>0)$, the state in each of these twodimensional Hilbert spaces can be represented as a Bloch vector with coordinates $\left(\varphi, \theta_{k}\right)$. A change in the parameter $\varphi$ determines a rotation of each Bloch vector about the $z$ direction. A closed circle will, therefore, produce an overall phase given by the sum of the individual phases as given in (31) and illustrated in Fig. 3(a). 
Of particular interest is the relative geometric phase between the first excited and ground states given by the difference of the Berry phases acquired by these two states. The first excited state is given by

$$
\left|e_{k_{0}}\right\rangle=|1\rangle_{k_{0}}|0\rangle_{-k_{0}} \bigotimes_{k \neq k_{0}}\left(\cos \frac{\theta_{k}}{2}|0\rangle_{k}|0\rangle_{-k}-i e^{i \varphi} \sin \frac{\theta_{k}}{2}|1\rangle_{k}|1\rangle_{-k}\right),
$$

with $k_{0}$ corresponding to the minimum value of the energy dispersion function $\varepsilon_{k}$. The behaviour of this state is similar to a direct product of only $n-1$ spins oriented along $\left(\varphi, \theta_{k}\right)$, where the state of the spin corresponding to momentum $k_{0}$ does not contribute any more to the geometric phase. Thus the relative geometric phase between the ground and the excited state becomes

$$
\phi_{e g}:=\phi_{e}-\phi_{g}=-\pi\left(1-\cos \theta_{k_{0}}\right) .
$$

In the thermodynamic limit $(N \rightarrow \infty), \phi_{\text {eg }}$ takes the form

$$
\phi_{e g}=\left\{\begin{array}{cl}
0, & \text { for }|h|>1-\delta^{2} \\
-\pi+\frac{\pi h \delta}{\sqrt{\left(1-\delta^{2}\right)\left(1-\delta^{2}-h^{2}\right)}}, & \text { for }|h|<1-\delta^{2}
\end{array}\right.
$$

where the condition $|h|>1-\delta^{2}$ constrains the excited state to be completely oriented along the $z$ direction resulting in a zero geometric phase. As can be seen from Fig. 3(b), the most interesting behaviour of $\varphi_{\text {eg }}$ is obtained in the case of $\delta$ small compared to $h$. In this case $\phi_{e g}$ behaves as a step function, giving either $\pi$ or 0 phase, depending on whether $|h|<1$ or $|h|>1$, respectively. This behaviour is precisely determined by the topological property of the corresponding loop, i.e. whether a critical point is encircled or not. This property can therefore be used to witness the presence of a criticality. More precisely, in the $|h|<1-\delta^{2}$ case, one can identify the first contribution with a purely topological term, while the second is an additional geometric contribution [30]. In other words, this first part gives rise to phase whose character depends only on whether the loops can be adiabatically shrunk to a point, i.e. on whether it is contractible. This is a purely topological character of the trajectory traced by the $\left(\varphi, \theta_{k}\right)$ coordinates. In particular if $n$ circulations are performed then the topological phase is $n \pi$, where $n$ is the winding number. The second term is geometric in nature and it can be made arbitrarily small by tuning appropriately the couplings $h$ or $\delta$. This idea is illustrated in Fig. 4, where the energy surface of ground and first excited state is depicted. The point of degeneracy is the intersection of the two surfaces. This is the point where the energy density is not analytical. Consider the case of a family of loops converging to a point. In the trivial case in which the limiting point does not coincide to a degeneracy, the corresponding Berry phase converges to zero. If instead, the degeneracy point is included, the Berry phase tends to a finite value [32].

To better understand the properties of the relative geometric phase, we focus on the region of parameters with $\delta \ll 1$. In this case, it can be shown [31] that the Hamiltonian, when restricted to its lowest energy modes, can be casted in a real form and, for $|h|<1$, its eigenvalues present a conical intersection centred at $\delta=0$. It is well known that, when a closed path is taken within the real domain of a Hamiltonian, a topological phase shift $\pi$ occurs only when a conical intersection is enclosed. In the present case, the conical intersection corresponds to a point of degeneracy where the XX criticality occurs and it is revealed by the topological term in the relative geometric phase $\phi_{\text {eg }}$. It is worth noticing that the presence of a conical intersection indicates that the energy gap scales linearly with respect to the coupling $\gamma$ when approaching the degeneracy point. This implies that the critical exponents of the energy, $z$, and of the correlation length, $v$, satisfy the relation $z v=1$ which is indeed the case for the XX criticality [7]. In the following we will see that geometric phases are sufficient to determine the exact values of the critical exponents and thus provide a complete characterisation of the criticality behaviour.

\subsection{General considerations}

One can show that the vacuum expectation value of a Hermitian operator, $O$, can be written in terms of a geometric phase. The only requirements posed on $O$ are that it should not commute with the Hamiltonian and it should be able to transform the ground state in a cyclic fashion. This is a rather general result that can be used to study the critical models.

To show that let us extend the initial Hamiltonian, $H_{0}$, of the model in the following way

$$
H=H_{0}+\lambda O .
$$

Turning to the interaction picture with respect to the $O$ term we obtain

$$
H_{\text {int }(\lambda)}=U(\lambda t) H_{0} U^{\dagger}(\lambda t),
$$

where $U(\lambda t)=\exp (-i \lambda O t)$. From the cyclicity requirement, there exists a time $T$ such that the unitary rotation $U(\lambda T)$ brings the ground state $|\psi\rangle$ to itself, i.e. $U(\lambda T)|\psi\rangle=|\psi\rangle$, producing eventually the desired cyclic evolution. The Berry phase that results from this evolution is given by Eq. (12), and thus, one obtains

$$
\varphi^{B}=-i \oint\left\langle\psi\left|U^{\dagger}(\lambda t) d U(\lambda t)\right| \psi\right\rangle=2 \pi i\langle\psi|O| \psi\rangle .
$$




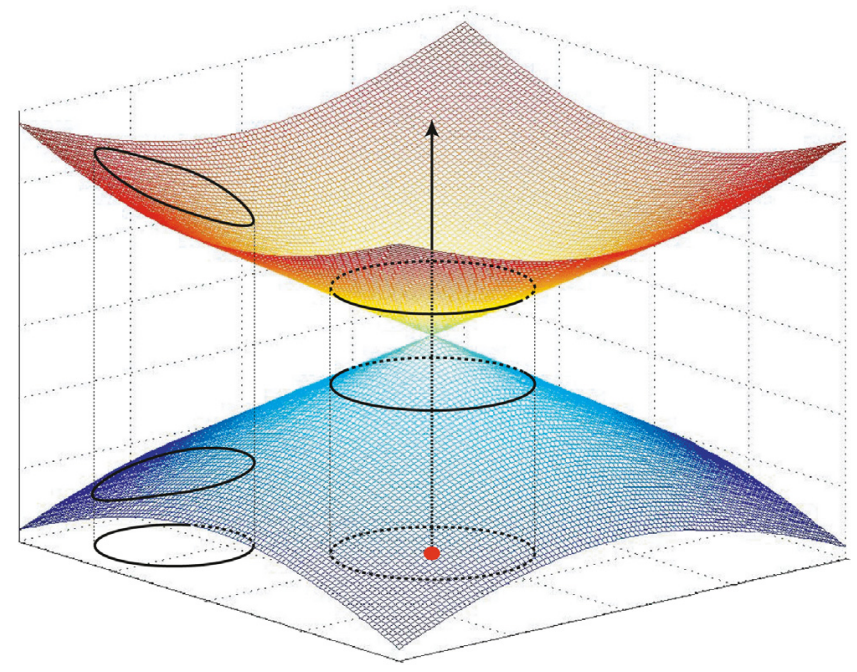

Fig. 4. The conical intersection between the two lowest energy levels of the Hamiltonian as a function of the parameters. A contractible loop, i.e. a loop that can be continuously deformed to a point of the domain, produces a zero geometric phase. A non-trivial geometric phase is obtained for non-contractible loops.

From this expression, we see that the geometric phase is a simple function of the vacuum expectation value of the operator that generates the circular paths. This expression can be easily inverted to finally give the expectation value of $O$ as a function of the geometric phase (37).

One can easily verify this relation for the simple case of a spin-1/2 particle in a magnetic field. When the direction of the magnetic field is changed adiabatically and isospectrally then the state of the spin is guided in a cyclic path around the $z$-direction. The generated phase is given by $\varphi_{B}=i 2 \pi \cos \theta$, where $\theta$ is the fixed direction of the magnetic field with respect to the $z$ direction. On the other hand, one can easily evaluate that the expectation value of the operator $\left(1-\sigma^{z}\right) / 2$ that generates the cyclic evolution is given by $\left\langle\psi\left|\left(1-\sigma^{z}\right) / 2\right| \psi\right\rangle=(1-\cos \theta) / 2$, which verifies relation (37), as for example $\lambda T=2 \pi$.

This connection has far reaching consequences. It is expected that intrinsic properties of the state will be reflected in the properties of the geometric phases. The latter, as they result from a physical evolution, can be obtained and measured in a conceptually straightforward way. To illustrate this we shall focus on critical phenomena in spin systems. Indeed, the presence of critical points can be detected by the behaviour of specific geometric evolutions and the corresponding critical exponents can be extracted. This comes as no surprise as one can choose geometric phases that correspond to the correlations of the system (expectation values of, e.g. $\sigma_{1}^{z} \sigma_{L}^{z}$ ) from where the correlation length and the critical behaviour can be obtained.

Let us apply this idea to the XY model, where the rotations are generated by the operator $0:=\sum_{j} \sigma_{j}^{z}$. One can easily see that the resulting geometric phase is proportional to the total magnetisation

$$
M_{z}=\left\langle\psi\left|\sum_{j} \sigma_{j}^{z}\right| \psi\right\rangle .
$$

It is well known [7] that the magnetisation $M_{z}$ can serve as an order parameter from which one can derive all the critical properties of the XY model just by considering its scaling behaviour. Indeed, Zhu [39] has considered the scaling of the ground-state geometric phase of the XY model from where he evaluated the Ising critical exponents. As it has been shown here, this is a general property that can be applied to any critical system.

\subsection{Dicke model and geometric phases}

In this section, we illustrate yet another model in which the properties of a quantum phase transitions can be observed and investigated through the geometric phase. We will discuss the thermodynamic and finite size scaling properties of the geometric phase in the adiabatic Dicke model (DM) [38], describing the super-radiant phase transitions for an $n$-qubit register coupled to a slow oscillator mode. One can show that, in the thermodynamic limit, the Berry phase has a topological feature similar to the one highlighted in the previous sections for the case of the XY model. A non-zero geometric phase is obtained only if a path in parameter space encircles the critical point. Furthermore, in this context one can show that precursors of this critical behaviour, for a system with finite size, exist and the scaling law of the Berry phase can be obtained in the leading order in the $1 / n$ expansion. 
Let us consider a system which consists of $n$ two-level systems, a qubit register or an ensamble of indistinguishable atoms, coupled to a single bosonic mode. The Hamiltonian, in unit such that $\hbar=c=1$, is given by

$$
H=\omega a^{\dagger} a+\Delta S_{x}+\frac{\lambda}{\sqrt{n}}\left(a^{\dagger}+a\right) S_{z},
$$

where $\Delta$ is the transitions frequency of the qubit, $\omega$ is the frequency of the oscillator and $\lambda$ is the coupling strength. The qubit operators are expressed in terms of total spin components $S_{k}=\sum_{j=1}^{n} \sigma_{j}^{k}$, where the $\sigma_{j}^{k}$ 's $(k=x, y, z)$ are the Pauli matrices used to describe the $j$ th qubit. A $\pi / 2$ rotation around the $y$ axis shows that $H$ is canonically equivalent to the standard formulation of the Dicke Hamiltonian [242], including counter-rotating terms.

After the first derivation due to Hepp and Lieb [243,244], the thermodynamic properties of the DM have been studied by many authors [245-251]. In the thermodynamic limit $(n \rightarrow \infty)$, the system exhibits a second-order phase transitions at the critical point $\lambda_{c}=\sqrt{\Delta \omega / 2}$, where the ground state changes from a normal to a super-radiant phase in which both the field occupation and the spin magnetisation acquire macroscopic values. The continued interest in DM stems from the fact that it displays a rich dynamics where many non-classical effects have been predicted [252-257], and from its broad range of applications [258]. Investigations on the ground state entanglement of the Dicke model have been also performed [259-261], pointing out a scaling behaviour around the critical point.

In this section we will outline the topological character of the geometric phase of the Dicke model in the adiabatic regime $(\Delta \gg \omega)$, and illustrate the scaling law of the geometric phase close to the critical point for a system with finite size.

In order to generate a Berry phase one can change the Hamiltonian by means of the unitary transformation:

$$
U(\varphi)=\exp \left(-i \frac{\varphi}{2} S_{x}\right),
$$

where $\varphi$ is a slowly varying parameter, moving from 0 to $2 \pi$. The transformed Hamiltonian can be written as

$$
H(\phi)=U^{\dagger}(\varphi) H U(\varphi)=\frac{\omega}{2}\left[p^{2}+q^{2}+\mathbf{Q} \cdot \mathbf{s}\right],
$$

where $\boldsymbol{Q}=\left(D, \frac{L q}{\sqrt{n}} \sin \varphi, \frac{L q}{\sqrt{n}} \cos \varphi\right)$ is an effective vector field. Here, $D=2 \Delta / \omega$ and $L=2 \sqrt{2} \lambda / \omega$ are dimensionless parameters, and the Hamiltonian of the free oscillator field is expressed in terms of canonical variables $q=\left(a^{\dagger}+a\right) / \sqrt{2}$ and $p=i\left(a^{\dagger}-a\right) / \sqrt{2}$, that obey the quantisation condition $[q, p]=i$.

In the adiabatic limit [262], where one assumes a slow oscillator and work in the regime $D \gg 1$, the Born-Oppenheimer approximation can be employed to write the ground state of $H(\phi)$ as:

$$
\left|\Psi_{\text {tot }}\right\rangle=\int d q \psi(q)|q\rangle \otimes|\chi(q, \phi)\rangle .
$$

Here, $|\chi(q, \phi)\rangle$ is the state of the "fast component"; namely, the lowest eigenstate of the "adiabatic" equation for the qubit part, displaying a parametric dependence on the slow oscillator variable $q$,

$$
\boldsymbol{Q} \cdot \mathbf{S}|\chi(q, \phi)\rangle=E_{l}(q)|\chi(q, \phi)\rangle \text {. }
$$

As the qubits are indistinguishable, it is easy to prove that the ground state can be expressed as a direct product of $n$ identical factors,

$$
|\chi(q, \phi)\rangle=|\chi(q, \phi)\rangle_{1} \otimes|\chi(q, \phi)\rangle_{2} \otimes \ldots \otimes|\chi(q, \phi)\rangle_{n} .
$$

Each component can be written as

$$
|\chi(q, \phi)\rangle_{j}=\sin \frac{\theta}{2}|\uparrow\rangle_{j}-\cos \frac{\theta}{2} e^{i \zeta}|\downarrow\rangle_{j},
$$

where $|\uparrow\rangle_{j}$ and $|\downarrow\rangle_{j}$ are the eigenstates of $\sigma_{j}^{z}$ with eigenvalues \pm 1 , and where

$$
\begin{aligned}
& \cos \theta:=\frac{L q \cos \varphi}{\sqrt{n} E(q)}, \\
& \zeta:=\arctan \frac{L q \sin \varphi}{\sqrt{n} D} .
\end{aligned}
$$

Here, $E(q)$ is related to the energy eigenvalue of Eq. (43) as

$$
E_{l}(q)=-n E(q)=-n \sqrt{D^{2}+\frac{L^{2} q^{2}}{n}} .
$$

In the Born-Oppenheimer approach, this energy eigenvalue constitutes an effective adiabatic potential felt by the oscillator together with the original square term

$$
V_{l}(q)=\frac{\omega}{2}\left[q^{2}-n E(q)\right] .
$$


Introducing the dimensionless parameter $\alpha=L^{2} / 2 D$, one can show that for $\alpha \leq 1$, the potential $V_{l}(q)$ can be viewed as a broadened harmonic well with minimum at $q=0$ and $V_{l}(0)=-n \Delta$. For $\alpha>1$, on the other hand, the coupling with the qubit splits the oscillator potential producing a symmetric double well with minima at $\pm q_{m}= \pm \frac{\sqrt{n} D}{L} \sqrt{\alpha^{2}-1}$ and $V_{l}\left(q_{m}\right)=-\frac{n \Delta}{2}\left(\alpha+\frac{1}{\alpha}\right)$.

As last step in the Born-Oppenheimer procedure, we need to evaluate the ground-state wave function for the oscillator, $\psi_{0}(q)$, that has to be inserted in Eq. (42) to obtain the ground state of the composite system. This wave function is the normalised solution of the one-dimensional time independent Schrödinger equation

$$
H_{a d} \psi_{0}(q)=\left(-\frac{\omega}{2} \frac{d^{2}}{d q^{2}}+V_{l}(q)\right) \psi_{0}(q)=\varepsilon_{0} \psi_{0}(q),
$$

where $\varepsilon_{0}$ is the lowest eigenvalues of the adiabatic Hamiltonian defined by the first equality.

Once this procedure is carried out for every value of the rotation angle $\phi$, the Berry phase of the ground state is obtained as

$$
\phi^{B}=i \int_{c} d \varphi\left\langle\Psi_{\text {tot }}\left|\frac{d}{d \varphi}\right| \Psi_{t o t}\right\rangle=\int_{-\infty}^{+\infty} d q \psi_{0}^{2}(q) \int_{0}^{2 \pi} d \varphi A^{B}(q, \varphi),
$$

where we introduced a q-parameterised Berry connection, given by

$$
A^{B}(q, \varphi):=i\left\langle\chi_{l}(q, \varphi)\left|\frac{d}{d \varphi}\right| \chi_{l}(q, \varphi)\right\rangle=-n \frac{d \zeta}{d \varphi} \cos ^{2} \frac{\theta}{2}=-\frac{n D}{2 E(q)} \frac{\frac{L q}{\sqrt{n}} \cos \varphi}{E(q)-\frac{L q}{\sqrt{n}} \cos \varphi} .
$$

Substituting this expression into Eq. (51), one finds

$$
\phi^{B}=n \pi\left(1+\frac{\left\langle S_{x}\right\rangle}{n}\right),
$$

where the average magnetisation per spin is

$$
\frac{\left\langle S_{x}\right\rangle}{n}=-\int_{-\infty}^{\infty} \psi_{0}^{2}(q) \frac{D}{E(q)} d q .
$$

Notice that Eq. (52) holds in general, its validity relying on the form of the rotation operator $U(\varphi)$ of Eq. (40) and not being restricted to the adiabatic regime. In the thermodynamic limit, one can show that

$$
\frac{\left\langle S_{x}\right\rangle}{n}= \begin{cases}-1 & (\alpha \leq 1) \\ -\frac{1}{\alpha} & (\alpha>1)\end{cases}
$$

and thus, for $n \rightarrow \infty$, the BP is given by [263]

$$
\left.\frac{\phi^{B}}{n}\right|_{n \rightarrow \infty}= \begin{cases}0 & (\alpha \leq 1) \\ \pi\left(1-\frac{1}{\alpha}\right) & (\alpha>1),\end{cases}
$$

It is worth stressing once again, that for the thermodynamic limit this result holds independently of the adiabatic approximation, whose use is needed here to obtain the finite-size behaviour. Numerical results for the scaled Berry phase are plotted in Fig. 5 as a function of the parameter $\alpha$, for $D=10$ and for different values of $n$, in comparison with the result for the thermodynamic limit. One can see that the Berry phase increases as the coupling strength growths and, in the thermodynamic limit, its derivative becomes discontinuous at the critical value $\alpha=\alpha_{c}:=1$. This result agrees with the expected behaviour of the geometric phase across the critical point. Notice that, in the thermodynamic limit, a non-trivial Berry phase is only obtained when a region of criticality is encircled, as for the path $C_{1}$ in Fig. 6 . Indeed, in the enlarged parameter space generated by the application of the unitary operator $U(\phi)$ of Eq. (40), the critical point corresponds to the paraboloid $\alpha=\frac{L^{2}}{2 D}=1$. As the radius of the path is determined by $\alpha$, one can see that, in the limit $n \rightarrow \infty$, the Berry phase is zero in the normal phase $(\alpha \leq 1)$ and is non-zero in the super-radiant phase, i.e. if the path encloses the critical region. This behaviour is indeed reminiscent of the topological features displayed by the geometric phase of the XY model described in the previous sections.

It is worth considering also the finite-scaling behaviour of the Berry phase at the critical point. In order to obtain an analytic estimation of Berry phase as a function of $n$, one can expand the adiabatic potential in Eq. (50) in powers of $\frac{1}{n D}$ and by using the expressions of the perturbation coefficients $c_{k}$, one obtains an anharmonic oscillator potential

$$
U_{l}(q)=\frac{2}{\omega} V_{l}(q) \simeq-n D+(1-\alpha) q^{2}+\frac{\alpha^{2}}{2 n D} q^{4} .
$$

The eigenvalue problem defined by this potential can be solved with the help of Symanzik scaling [263,264]. This is done by rewriting Eq. (50) into the equivalent form

$$
\left[-\frac{d^{2}}{d x^{2}}+\mu x^{2}+x^{4}\right] \psi_{0}(x ; \mu)=e_{0}(\mu) \psi_{0}(x ; \mu),
$$




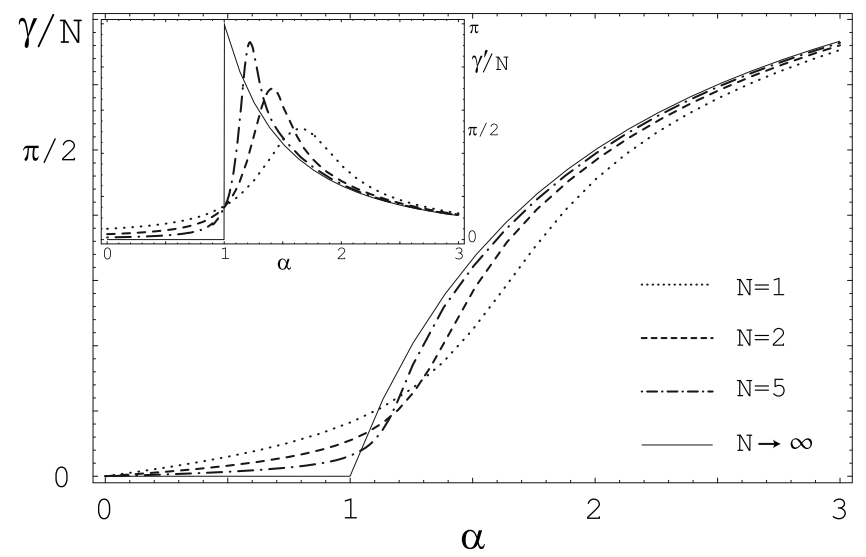

Fig. 5. Numerical results for the scaled Berry phase as a function of the parameter $\alpha$, for $D=10$ and for different values of $n$, in comparison with the result for $n \rightarrow \infty$. Berry's phase increases with the coupling, and, in the thermodynamic limit, its derivative becomes discontinuous at the critical value $\alpha=\alpha_{c}:=1$. The inset shows the derivative of the Berry phase with respect to $\alpha$.

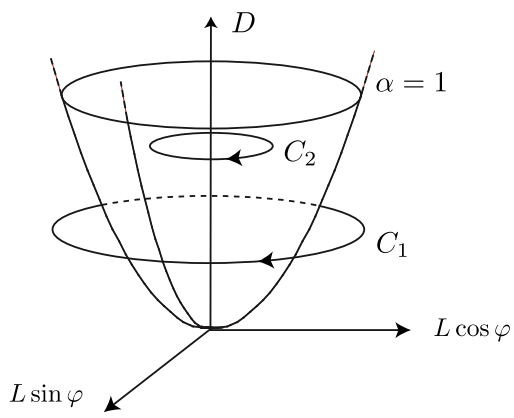

Fig. 6. A qualitative illustration of the paths followed by the parameters of the Hamiltonian due to the application of $U(\phi)$. The paraboloid corresponds to the value $\alpha=L^{2} / 2 D=1$, for which the Hamiltonian shows a critical behaviour. If the parameters follow a path, e.g. $C_{1}$, encircling the paraboloid, then the system acquires a non-trivial Berry phase, which tends to $\pi$ for $\alpha \gg 1$. The path $C_{2}$ gives rise to a zero Berry phase (in the thermodynamic limit).

where the scaled position is $x:=q\left(\frac{\alpha^{2}}{2 n D}\right)^{1 / 6}$, while $\mu:=\left(\frac{2 n D}{\alpha^{2}}\right)^{2 / 3}\left(\alpha_{c}-\alpha\right)$. Finally, the energies in Eqs. (50) and (57) obey the scaling relation

$$
\frac{2}{\omega} \varepsilon_{0}(\alpha, n D)=-n D+\left(\frac{\alpha^{2}}{2 n D}\right)^{1 / 3} e_{0}(\mu) .
$$

Since $\mu \rightarrow 0$ at the critical point, we can consider the $x^{2}$ term to be a perturbation and employ the Rayleigh-Schrödinger perturbation theory. This yields the expansion $e_{0}(\mu)=\sum_{k=0}^{\infty} c_{k} \mu^{k}$, where the coefficients $c_{k}$ can be obtained after solving the equation for a purely quartic oscillator. It is easy to get $c_{0}=e_{0}(0) \simeq 1.06036$ and $c_{1}=\int_{-\infty}^{\infty} q^{2} \phi_{0}^{2}(q ; 0) d q=e_{0}^{\prime}(0) \simeq$ 0.36203 .

It can be shown that the coefficients in the expansion of $e_{0}(\mu)$ completely determine the average value of every physical observable at the critical point [263]. In particular, a similar expansion applied to $\left\langle S_{x}\right\rangle$ allows one to write [38]

$$
\frac{\left\langle S_{x}\right\rangle}{n} \simeq-1+\frac{2 c_{1}}{(2 n D)^{2 / 3}}-\frac{2 c_{0}}{(2 n D)^{4 / 3}} .
$$

Thus, one obtains the leading orders in the finite size scaling of the Berry phase as

$$
\frac{\phi^{B}}{n} \approx \pi\left[\frac{2 c_{1}}{(2 n D)^{2 / 3}}-\frac{2 c_{0}}{(2 n D)^{4 / 3}}\right] .
$$

This expression shows how the scaled geometric phase goes to zero as $n$ increases and how the singular thermodynamic behaviour is approached at $\alpha=\alpha_{c}=1$. The leading critical behaviour of the Berry phase, $\phi^{B} / n \sim n^{-2 / 3}$ is confirmed in Fig. 7 by comparison with the numerical evaluation of the geometric phase obtained from Eqs. (52)-(53). In fact, including also the second order correction, scaling as $n^{-4 / 3}$, reproduces the numerical results even for small values of $n$. 


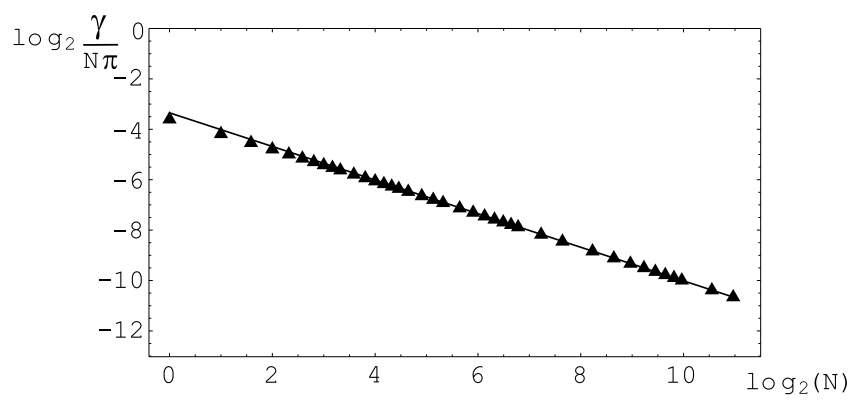

Fig. 7. Scaling of the Berry phase as a function of $n$ at the critical point $\alpha=1$, for $D=10$. For ease of comparison, the continuous plot shows the analytic expression of Eq. (60).

Besides the scaling relation at the critical point $\alpha=1$, one can also obtain the leading $1 / n$ correction to the thermodynamic limit of $\frac{\phi^{B}}{n}$ for small and large values of $\alpha$, i.e., for path of very small and very large radii [38]. Since the oscillator localises around $q=0$ for $\alpha \ll 1$, while its wave function is split in two components peaked around $\pm q_{m}$ for $\alpha \gg 1$, one gets [38]

$$
\frac{\phi^{B}}{n}-\left.\frac{\phi^{B}}{n}\right|_{n \rightarrow \infty} \approx \begin{cases}\frac{\pi \alpha}{2 n D} & (\alpha \ll 1) \\ -\frac{\pi}{n D \alpha^{2}} & (\alpha \gg 1) .\end{cases}
$$

Thus, far from the critical point (that is, well inside the normal and super-radiant phase), the Berry phase per qubit reaches its thermodynamic limit as $n^{-1}$. Therefore, the topological behaviour of $\phi^{B}$ can be inferred even for a relatively small number of qubits. To summarise, this example shows that the behaviour of the geometric phase in correspondence of the critical region for the Dicke model, confirms the general connection between geometric phase and quantum critical phase transitions. Indeed, geometric phase and QPTs share the common feature of both appearing in presence of a singularity in the energy density of the system. This heuristic argument motivates - once again - the need to explore the use of Berry phase as a tool to signal and investigate critical features of certain models. However, strictly speaking, singularities in the energy density of many body systems only appear in the thermodynamic limit. It is therefore interesting to notice that, in a finite scale regime, such a connection between Berry phase and QPTs can still be drawn, owing to the geometric phase sensitivity to the increase of the parametric manifold curvature, as the thermodynamic limit is approached. Studying the Berry phase in this regime has clearly theoretical interest and obvious experimental motivations. In the case of the Dicke model, it is found that the geometric phase start to show, already at finite sizes, the topological character which is typically manifested at the thermodynamic limit. Moreover, studying the scaling of the Berry phase as a function of the system size, one can identify its critical exponent.

\section{Quantum phase transition and information geometry}

For the sake of completeness, and to clearly pave the ground to the last part of this review, we will introduce another approach to the study of quantum phase transitions, i.e. the so called fidelity approach $[158,159,193,194]$, which has been successfully employed in classical [162-164] and quantum phase transitions [42,158-161,198], both in symmetrybreaking [42,158-161,165,166] as well as in topological phase transitions [167]. As a distance measure, the fidelity describes how close two given quantum states are. Therefore, it is natural to expect that the fidelity can be used to characterise the drastic changes occurring in quantum many body states going through QPTs, regardless of what type of order parameters, if any, characterises the type of phase transitions. In the fidelity approach, quantum phase transitions are identified by studying the behaviour of the overlap between two ground states corresponding to two slightly different set of parameters; at QPTs a drop of the fidelity with scaling behaviour is observed and quantitative information about critical exponents can be extracted [42]. As for the geometric phase approach, the fidelity approach neither is based on the knowledge of an order parameter, nor it requires a notions of symmetry breaking. It is indeed an approach purely based on the distinguishability of pure states, as well as density matrices, and it does not even require the knowledge of the Hamiltonian itself. In a sense, it is a purely kinetic approach, as opposed to the traditional methods which rely on the information derived from the dynamics of the many-body systems.

\subsection{Metric on the Hilbert space: the Fubini-Study metric}

In a given Hilbert space, there is a natural gauge invariant metric that can be defined in terms of elements of the projective Hilbert space $\mathcal{P}$. Given any two $\psi_{1}$ and $\psi_{2}$ in the Hilbert space, the distance $d_{F S}$ between any two points $p_{1}:=\left|\psi_{1}\right\rangle\left\langle\psi_{1}\right|$ and $p_{2}:=\left|\psi_{2}\right\rangle\left\langle\psi_{2}\right|$ in $\mathcal{P}$ is defined by

$$
d_{F S}\left(p_{1}, p_{2}\right)=\inf _{\alpha_{1}, \alpha_{2}}\left\|e^{i \alpha_{1}} \psi_{1}-e^{i \alpha_{2}} \psi_{2}\right\|=\sqrt{2-2\left|\left\langle\psi_{1} \mid \psi_{2}\right\rangle\right|}
$$


where minimisation is obtained when $e^{i \alpha_{1}} \psi_{1}$ and $e^{i \alpha_{2}} \psi_{2}$ are in phase, according to Pancharatnam's criterion, i.e. when $\left\langle e^{i \alpha_{1}} \psi_{1} \mid e^{i \alpha_{2}} \psi_{2}\right\rangle$ is a real and positive number. Clearly, $d_{F B}\left(p_{1}, p_{2}\right) \geq 0$ with equality holding if and only if $p_{1}=p_{2}$. Also $d_{F S}\left(p_{1}, p_{2}\right)=d_{F S}\left(p_{2}, p_{1}\right)$. The triangle inequality, for any $p_{1}=\left|\psi_{1}\right\rangle\left\langle\psi_{1}\left|, p_{2}=\right| \psi_{2}\right\rangle\left\langle\psi_{2}\right|$ and $p_{3}=\left|\psi_{3}\right\rangle\left\langle\psi_{3}\right|$ in $\mathcal{P}$, is implied by the following chain of relations

$$
d_{F S}\left(p_{1}, p_{2}\right)+d_{F S}\left(p_{2}, p_{3}\right)=\left\|\psi_{1}-\psi_{2}\right\|+\left\|\psi_{2}-\psi_{3}\right\| \geq\left\|\psi_{1}-\psi_{3}\right\| \geq d_{F S}\left(p_{1}, p_{3}\right),
$$

where $\psi_{2}$ is in phase with $\psi_{1}$, and $\psi_{3}$ with $\psi_{2}$. Hence $d_{F S}$ is a metric on $\mathcal{P}$, called the Fubini-Study distance [265,266], which can be expressed as $d_{F B}\left(p_{1}, p_{2}\right)^{2}=2\left(1-\sqrt{\operatorname{Pr}\left(p_{1}, p_{2}\right)}\right)$, where $\operatorname{Pr}\left(p_{1}, p_{2}\right):=\left|\left\langle\psi_{1} \mid \psi_{2}\right\rangle\right|^{2}$ is the so called transitions probability. The latter quantifies the probability to get an affirmative answer when testing whether the system is in the state $p_{1}$ if it was actually in state $p_{2}$, or vice versa. In other words, it quantifies the statistical distinguishability between pure states. The Fubini-Study distance is indeed a geometrical measure of statistical indistinguishability between pure quantum states $[21,22,157]$. In terms of $d_{F S}\left(p_{1}, p_{2}\right)$, the projective space, corresponding to the subspace spanned by $\psi_{1}$ and $\psi_{2}$, is described by a 2-sphere with unit radius embedded in a 3-dimensional Euclidian space. In such a sphere, $d_{F S}\left(p_{1}, p_{2}\right)$ is the straight-line distance separating $p_{1}$ and $p_{2}$.

Suppose that $\psi_{1}$ and $\psi_{2}$ are such that $\Pi\left(\psi_{1}\right)$ and $\Pi\left(\psi_{2}\right)$ are infinitely close in $\mathcal{P}$. Then Eq. (62) defines a Riemannian metric on $\mathcal{P}$ called the Fubini-Study metric. To obtains its metric coefficients, consider a curve $\mathcal{C}$ in $\mathcal{P}$ parameterised in the interval $\left[s_{a}, s_{b}\right]$, and let $C:=\psi(s)$ be any of its lift in the Hilbert space. By Taylor expanding,

$$
\langle\psi(s) \mid \psi(s+d s)\rangle=1+\langle\psi \mid \dot{\psi}\rangle d s+\frac{1}{2}\langle\psi \mid \ddot{\psi}\rangle d s^{2}+\mathcal{O}\left(d s^{3}\right),
$$

where $\dot{\psi}:=d \psi / d s$. Also, differentiating $\langle\psi(s) \mid \psi(s)\rangle=1$ twice yields

$$
\begin{aligned}
\langle\psi \mid \dot{\psi}\rangle+\langle\dot{\psi} \mid \psi\rangle & =0, \\
\langle\psi \mid \ddot{\psi}\rangle+\langle\ddot{\psi} \mid \psi\rangle+2\langle\dot{\psi} \mid \dot{\psi}\rangle & =0,
\end{aligned}
$$

hence,

$$
\begin{aligned}
d l^{2} & :=2\left(1-\sqrt{|\langle\psi(s) \mid \psi(s+d s)\rangle|^{2}}\right) \\
& =2-2 \sqrt{1+(\langle\dot{\psi} \mid \psi\rangle+\langle\psi \mid \dot{\psi}\rangle) d s+\left(\langle\dot{\psi} \mid \psi\rangle\langle\psi \mid \dot{\psi}\rangle+\frac{1}{2}\langle\ddot{\psi} \mid \psi\rangle+\frac{1}{2}\langle\psi \mid \ddot{\psi}\rangle\right) d s^{2}} \\
& =\langle\dot{\psi}|(\mathbb{1}-|\psi\rangle\langle\psi|)| \dot{\psi}\rangle d s^{2} \\
& =\sum_{\mu \nu} g_{\mu \nu} d \lambda_{\mu} d \lambda_{\nu},
\end{aligned}
$$

where $\left\{\lambda_{\mu}\right\} \in \mathcal{M}$ are a set of local parameters labelling $\mathcal{P}$ in the neighbourhood of $\Pi(\psi)$, and

$$
g_{\mu \nu}:=\operatorname{Re} Q_{\mu \nu}
$$

is a positive-definite real matrix, where

$$
Q_{\mu \nu}:=\left\langle\partial_{\mu} \psi|(\mathbb{1}-|\psi\rangle\langle\psi|)| \partial_{\nu} \psi\right\rangle
$$

is a positive semi-definite Hermitian matrix, called the quantum geometric tensor (QGT) $[28,267]$. This quantity, by definition, is gauge invariant, and its imaginary part coincides, up to a factor $1 / 2$, to the Berry curvature

$$
\operatorname{Im} Q_{\mu \nu}=\frac{\left\langle\partial_{\mu} \psi \mid \partial_{\nu} \psi\right\rangle-\left\langle\partial_{\mu} \psi \mid \partial_{\nu} \psi\right\rangle}{2}=\frac{F_{\mu \nu}^{B}}{2} .
$$

Notice that, the Fubini-Study metric can also be expressed as $d l_{F B}^{2}=\langle u(s) \mid u(s)\rangle d s^{2}$, where $|u\rangle=|\dot{\psi}\rangle-\langle\psi \mid \dot{\psi}\rangle|\psi\rangle$ is the component of the tangent vector $\dot{\psi}(s)$ orthogonal to $\psi(s)$. Alternatively,

$$
|u\rangle=\mathcal{D}_{s}|\psi\rangle:=|\dot{\psi}\rangle+i A_{s}^{B}|\psi\rangle,
$$

where $\mathcal{D}_{s}:=d / d s+i A_{s}^{B}$ is the covariant derivative and $A_{s}^{B}=i\langle\psi \mid \dot{\psi}\rangle$ is the Berry connection. Using this metric one can derive the geodesics in the space $\mathcal{P}$, which amounts to finding the path connecting two states which minimises the following length:

$$
D_{F S}:=\min \int_{\Pi\left(\psi\left(s_{a}\right)\right)}^{\Pi\left(\psi\left(s_{a}\right)\right)} d l_{F S}=\int_{s_{a}}^{s_{b}} \sqrt{\langle u(s) \mid u(s)\rangle} d s .
$$

It turns out that the length of the geodesics arc, connecting the two end points $\psi_{1}$ and $\psi_{2}$, is given by

$$
D_{F S}\left(p_{1}, p_{2}\right)=\int_{\mathcal{C}} d l_{F S}=\arccos \left|\left\langle\psi_{1} \mid \psi_{2}\right\rangle\right|=\arccos \sqrt{\operatorname{Pr}\left(p_{1}, p_{2}\right)},
$$

which is called Fubini-Study length or Fubini-Study angle, which is itself a distance on the projective Hilbert space. 


\subsection{Fubini-study distance as a statistical distance}

Let us digress onto a specific aspect of the Fubini-Study distance, which provides a measure of statistical (in-)distinguishability between pure quantum states. Assume that one wishes to perform a finite set of experiments to distinguish between two states $\psi_{1}$ and $\psi_{2}$. To this extent, one needs some specific set of measurements, or equivalently a set of observables, and then use the results to define a statistical distance between the states. However, it is clear that this distance will depend on the choice of the observable as well as on the states. A solution to this problem would be to single out an exceptional set of measurements which maximises the resulting statistical distance. By definition this will be the distance between the states.

Assume that a chosen observable $O$ has $n$ non-degenerate orthogonal eigenstates $|k\rangle$ in terms of which we can expand both states. When the state is $\psi_{i}$, according to the standard Born rule, the probability distribution $P_{i}:=\left\{p_{i}(k), k=1 \ldots n\right\}$ to obtain the $k$ th outcome in the measurement is $p_{i}(k):=\left|\left\langle\psi_{i} \mid k\right\rangle\right|^{2}$. Each state $\psi_{i}$, therefore, results in a distinct outcome probability distribution. The point now is to quantify by means of a statistical distance the degree of distinguishability of these probability distributions in an operationally meaningful way. Two popular choices that accomplish this task are the Bhattacharyya distance,

$$
D_{O}^{\text {Bha }}\left(\psi_{1}, \psi_{2}\right):=\arccos \left(\sum_{k} \sqrt{p_{1}(k) p_{2}(k)}\right)=\arccos B\left(P_{1}, P_{2}\right)
$$

and the Hellinger distance

$$
D_{O}^{H}\left(\psi_{1}, \psi_{2}\right):=\left(\sum_{k}\left(\sqrt{p_{1}(k)}-\sqrt{p_{2}(k)}\right)^{2}\right)^{\frac{1}{2}}=\sqrt{2-2 B\left(P_{1}, P_{2}\right)}
$$

both monotonous functions of the Bhattacharyya coefficient, which can be computed from the square roots of the probabilities,

$$
B\left(P_{1}, P_{2}\right):=\sum_{k} \sqrt{p_{1}(k) p_{2}(k)}=\sum_{k}\left|\left\langle\psi_{1} \mid k\right\rangle\right|\left|\left\langle k \mid \psi_{2}\right\rangle\right| \leq\left|\left\langle\psi_{1} \mid \psi_{2}\right\rangle\right| .
$$

There are several optimal measurements that saturate the inequality above. A solution is found by any observable 0 having either $\psi_{1}$ or $\psi_{2}$ as its eigenstate, in which case the Bhattacharyya distance and the Hellinger distance collapse to the Fubini-Study length $D_{F B}(72)$ and Fubini-Study distance $d_{F B}(62)$, respectively. This establishes the Fubini-Study metric as a measure of the distinguishability of pure quantum states in the sense of statistical distance [21]. More precisely, what the Fubini-Study distance measures is the experimental distinguishability of two quantum states, assuming no limitations on the type of measurements one can perform. In practise, a measurement device available to a laboratory may correspond to a limited subset of observables only, and this device may be subject to various sources of imperfections. In this case, the Fubini-Study geometry may not provide the correct measure of experimental distinguishability, but still it is relevant, as it provides information on what we can know in general, without knowledge of the specific physical system.

\subsection{Fubini-study metric and quantum phase transitions}

Let us consider a smooth family $H(\lambda)$ of Hamiltonians labelled by a set of parameters in a manifold $\lambda \in \mathcal{M}$, in the Hilbert-space $\mathcal{H}$ of the system. If $\left|\Psi_{0}(\lambda)\right\rangle \in \mathcal{H}$ denotes the, unique for simplicity, ground-state of $H(\lambda)$, one defines a oneto-one mapping $\Psi_{0}: \mathcal{M} \rightarrow \mathcal{H} / \lambda \rightarrow\left|\Psi_{0}(\lambda)\right\rangle$ associating to each set of parameters the ground-state of the corresponding Hamiltonian. This map can be seen also as a map between a point in $\mathcal{M}$ and an element of projective space $P \mathcal{H}$. As already pointed out in the previous subsections, the projective Hilbert space is equipped with a metric, the Fubini-Study distance

$$
d_{F S}\left(\psi, \psi^{\prime}\right):=\sqrt{2-2\left|\left\langle\psi, \psi^{\prime}\right\rangle\right|},
$$

which quantifies the maximum amount of statistical distinguishability between the pure states $|\psi\rangle$ and $\left|\psi^{\prime}\right\rangle$. It provides the statistical distance between the probability distributions of the outcomes associated to $|\psi\rangle$ and $\left|\psi^{\prime}\right\rangle$, optimised over all possible measurement strategies. Moreover, as we have already seen, this result easily extends to mixed states, by replacing the Fubini-Study distance with its natural density matrix generalisation, the Bures distance.

These non-trivial notions allow one to identify the projective Hilbert space geometry with a geometry in the information space, the larger the distance between $|\psi\rangle$ and $\left|\psi^{\prime}\right\rangle$ the more statistically distinguishable two states are. The remarkable consequences of this simple observation is that the distance encodes information on any of the infinitely many possible order parameters characterising the phase transitions. At the critical point, a small difference between the parameters labelling the Hamiltonian results in a greatly enhanced distinguishability of the corresponding ground states. This is quantitatively revealed by the state overlap and in turn by the behaviour of the metric. 
Let $\psi$ and $\psi+d \psi$ be two infinitesimally closed states in the parameter manifold. In Section 6.1, it has been shown that their elementary distance in the parameter space can be expressed as:

$$
d_{F B}^{2}(\psi, \psi+d \psi)=d l^{2}=\sum_{\mu \nu} g_{\mu \nu} d \lambda_{\mu} d \lambda_{n u}
$$

where metric tensor $g_{\mu \nu}=\mathbf{R e} Q_{\mu \nu}$ is the real part of the quantum geometric tensor $Q_{\mu \nu}$ introduced in Section 6.1, i.e.

$$
Q_{\mu \nu}=\left\langle\partial_{\mu} \psi \mid \partial_{\nu} \psi\right\rangle-\left\langle\partial_{\mu} \psi \mid \psi\right\rangle\left\langle\psi \mid \partial_{\nu} \psi\right\rangle \text {. }
$$

As explicitly pointed out in Section 6.1, the imaginary part of the quantum geometric tensor is $\operatorname{Im} Q_{\mu \nu}=\frac{F_{\mu \nu}}{2}$, where $F_{\mu \nu}$ is nothing but the Berry curvature 2-form. This provides a unifying framework for the understanding of the fidelity and geometric phase approaches to the quantum phase transitions.

A perturbative expansion provides a simple heuristic explanation as to why one observes a singular behaviour of the quantum geometric tensor at QPTs. By using the first order perturbative expansion

$$
\left|\Psi_{0}(\lambda+\delta \lambda)\right\rangle \sim\left|\Psi_{0}(\lambda)\right\rangle+\sum_{n \neq 0}\left(E_{0}(\lambda)-E_{n}(\lambda+d \lambda)\right)^{-1}\left|\Psi_{n}(\lambda)\right\rangle\left\langle\Psi_{n}(\lambda)|\delta H| \Psi_{0}(\lambda)\right\rangle,
$$

where $\delta H:=H(\lambda+\delta \lambda)-H(\lambda)$, one obtains for the entries of the quantum geometric tensor (78) the following expression

$$
Q_{\mu \nu}(\lambda)=\sum_{n \neq 0} \frac{\left\langle\Psi_{0}(\lambda)\left|\partial_{\mu} H\right| \Psi_{n}(\lambda)\right\rangle\left\langle\Psi_{n}(\lambda)\left|\partial_{\nu} H\right| \Psi_{0}(\lambda)\right\rangle}{\left[E_{n}(\lambda)-E_{0}(\lambda)\right]^{2}} .
$$

Continuous QPTs occur when, for some specific values of the parameters, the energy gap

$$
\Delta_{n}\left(\lambda_{c}\right):=E_{n}\left(\lambda_{c}\right)-E_{0}\left(\lambda_{c}\right) \geq 0
$$

vanishes in the thermodynamic limit. This amounts to a vanishing denominator in Eq. (80) that may break down the analyticity of the metric tensor entries. This heuristic argument has been first put forward in [159] specifically for the Riemannian tensor $g_{\mu \nu}$ and for the Berry Curvature $F_{\mu \nu}$ in [32]. An argument based on more firm grounds can also be formulated in terms of scaling properties of the quantum geometric tensor [42].

To get further insight about the physical origin of these singularities we notice that the metric tensor (78) can be cast in an interesting covariance matrix form [267]. Generically, changing the Hamiltonian from $H(\lambda)$ to $H(\lambda+\delta \lambda)$ within the same phase, no level-crossings occur. In this case, the unitary operator $U(\lambda, \delta \lambda):=\sum_{n}\left|\Psi_{n}(\lambda+\delta \lambda)\right\rangle\left\langle\Psi_{n}(\lambda)\right|$ can adiabatically map the eigenspace at the point $\lambda \in \mathcal{M}$ onto those at $\lambda+\delta \lambda$. In terms of the corresponding Hermitian generators $X_{\mu}:=i\left(\partial_{\mu} U\right) U^{\dagger}$, the Fubini-Study metric tensor (78) takes the form

$$
g_{\mu \nu}=(1 / 2)\left\langle\left\{\bar{X}_{\mu}, \bar{X}_{v}\right\}\right\rangle,
$$

where $\bar{X}_{\mu}:=X_{\mu}-\left\langle X_{\mu}\right\rangle$. In other words, $g_{\mu \nu}$ can be identified with the (symmetric) covariance matrix of the observables $X_{\mu}$. The differential line element $d l^{2}$ can be expressed as the variance of the operator-valued differential one-form $\boldsymbol{X}:=i(d U) U^{\dagger}=\sum_{\mu} X_{\mu} d \lambda_{\mu}$, i.e., $d l^{2}=\left\langle\bar{X}^{2}\right\rangle$. The operator $\boldsymbol{X}$ is the generator of the mapping between sets of eigenbases corresponding to infinitesimally closed points $\lambda$ and $\lambda+d \lambda$. The smaller the difference between these eigenbases for a given parameter variation, the smaller the variance of $\boldsymbol{X}$. At QPTs one expects to have the maximal possible difference between $\left|\Psi_{0}(\lambda)\right\rangle$ and $\left|\Psi_{0}(\lambda+\delta \lambda)\right\rangle$, i.e., many "unperturbed" eigenstates $\left|\Psi_{n}(\lambda)\right\rangle$ are needed to build up the "new" GS; accordingly the variance of $\boldsymbol{X}$ can get very large, possibly divergent. In this sense, $\boldsymbol{X}$ acquires the significance of an order parameter, and $d l^{2}$ can be interpreted as its susceptibility.

\subsection{Quantum phase transition and super-extensitivity of the quantum geometric tensor}

In this section we will derive a bound useful to establish a connection between the quantum geometric tensor $Q$ and QPTs. Let us consider a system of size $L^{d}$ (with dimensionality $d$ ). Since $Q(\lambda)$ is a Hermitian non-negative matrix one has

$$
\left|Q_{\mu \nu}\right| \leq\|Q\|_{\infty}=\boldsymbol{u}^{\dagger} \cdot Q \cdot \boldsymbol{u},
$$

where $\|B\|_{\infty}$ stands for the largest singular value of a matrix $B$ and $\boldsymbol{u}=\left\{u_{\mu}\right\}_{\mu=1}^{\operatorname{dim} \mathcal{M}}$, with $\boldsymbol{u}^{\dagger} \cdot \boldsymbol{u}=1$, is the normalised eigenvector of $Q$ with the largest eigenvalue. One can define the corresponding combination of parameters $\bar{\lambda}:=\sum_{\mu} u_{\mu} \lambda_{\mu}$, which, loosely speaking, is the direction on the parameter manifold encoding the maximal "responsiveness" of the geometry. Let us denote $\bar{\partial}:=\partial / \partial \bar{\lambda}$, then

$$
\bar{\partial} H=\sum_{\mu}\left(\partial_{\mu} H\right) u_{\mu} .
$$


From Eq. (80) and the above inequality (83),

$$
\begin{aligned}
\left|Q_{\mu \nu}\right| & \leq \sum_{n>0} \Delta_{n}^{-2}\left|\left\langle\Psi_{0}|\bar{\partial} H| \Psi_{n}\right\rangle\right|^{2} \leq \Delta_{1}^{-2} \sum_{n>0}\left|\left\langle\Psi_{0}|\bar{\partial} H| \Psi_{n}\right\rangle\right|^{2} \\
& =\Delta_{1}^{-2}\left(\left\langle\bar{\partial} H \bar{\partial} H^{\dagger}\right\rangle-|\langle\bar{\partial} H\rangle|^{2}\right),
\end{aligned}
$$

where the angular brackets denote the average over $\left|\Psi_{0}(\lambda)\right\rangle$. Now, a crucial assumption is that the operator $\bar{\partial} H$ is local i.e., $\bar{\partial} H=\sum_{j} \bar{\partial} V_{j}$ where $V_{j}$ are operators with local support around the site $j$. Then the last term in Eq. (85) reads

$$
\sum_{i, j}\left(\left\langle\bar{\partial} V_{i} \bar{\partial} V_{j}^{\dagger}\right\rangle-\left\langle\bar{\partial} V_{i}\right\rangle\left\langle\bar{\partial} V_{j}^{\dagger}\right\rangle\right)
$$

If the ground state is translationally invariant, this last quantity can be written as $L^{d} \sum_{r} K(r):=L^{d} K$, where

$$
K(r):=\left\langle\bar{\partial} V_{i} \bar{\partial} V_{i+r}^{\dagger}\right\rangle-\left\langle\bar{\partial} V_{i}\right\rangle\left\langle\bar{\partial} V_{i+r}^{\dagger}\right\rangle
$$

is independent of $i$. For gapped systems, i.e. $\Delta_{1}(\infty):=\lim _{L \rightarrow \infty} \Delta_{1}(L)>0$, the correlation function $G(r)$ is rapidly decaying [268] and therefore $K$ is finite and independent of the system size. Using (85) it follows that for these non-critical systems $\left|Q_{\mu \nu}\right|$ cannot grow, as a function of $L$, more than extensively. Indeed one has that

$$
\lim _{L \rightarrow \infty}\left|Q_{\mu \nu}\right| / L^{d} \leq K \Delta_{1}^{-2}(\infty)<\infty .
$$

Conversely if

$$
\lim _{L \rightarrow \infty}\left|Q_{\mu \nu}\right| / L^{d}=\infty,
$$

i.e. $\left|Q_{\mu \nu}\right|$ grows super-extensively, then either $\Delta_{1}(L) \rightarrow 0$ or $K$ cannot be finite. In both cases the system has to be gapless. Summarising: a super-extensive behaviour of any of the components of $Q$ for systems with local interaction implies a vanishing gap in the thermodynamic limit [42].

This behaviour has been observed in a variety of systems [158,159,193,194], and amounts to a critical fidelity drop at the QPTs. The extensive behaviour of the fidelity drop within a normal phase is strongly reminiscent of a well known physical phenomenon, the Anderson orthogonality catastrophe [197]. Namely, as the dimension of the system increases, the fidelity, i.e. the overlap, between two infinitesimally neighbouring ground states $\Psi_{0}(\lambda)$ and $\Psi_{0}(\lambda+d \lambda)$ approaches zero, no matter how small the difference in parameters $\lambda$ is, so that two ground states are mutually orthogonal in the thermodynamic limit.

This is a well known feature of systems in many-body physics having infinitely many degrees of freedom [197]. The fact that two physical states corresponding to two arbitrarily close sets of parameters, two arbitrarily similar physical situations, must become orthogonal to each other in the thermodynamic limit, is not a distinctive feature of a critical point. It is indeed a behaviour which is present across the whole phase diagram, hence also between two states belonging to the same phase. Hence, in itself, this characteristic has little to do with QPTs. Despite its emphatic expression, the "orthogonality catastrophe" is much less a dramatic and unusual peculiarity as its name would suggest. From a quantum information perspective, it is easier to appreciate how typical such a behaviour must be, given the infinite dimensionality of the Hilbert-space that many-body states explore.

What is indeed qualitatively different in QPTs is the rate at which the fidelity vanishes in the thermodynamic limit. It is only the presence of a dramatic, large scale change in the ground state properties of the system which may allow for a super-extensive increase of the metric, and the consequent rate of reduction of the state overlap. Loosely speaking, a criticality results in an orthogonality catastrophe that is expressed on a qualitatively greater scale. The intuition behind this change of scale may be gleaned as follows. A local perturbation to a many-body Hamiltonian far from a critical point may only result in local modifications to the state of the system, i.e. modifications which are within a region of the size of the correlation length $\xi$. Such local changes contribute to the reduction of the fidelity with infinitesimal amounts, that, when accrued, are enough to provide an increase in the total metric with a rate of up to $L^{d}$ in the system size $L$. A higher rate is only possible when a local perturbation generates non-local changes on the system states, i.e. when the correlation length $\xi$ diverges and the response of the system to a local perturbation brings in contributions from degrees of freedom at every scale.

In the following section, we will briefly illustrate the above considerations by using the simple, yet physically relevant, many body Hamiltonian: the $X Y$ spin-chain model.

\section{5. $X Y$ model and information geometry}

To illustrate explicitly how divergencies of $g_{\mu \nu}$ may arise [159], let us go back to the XY model already discussed in Section 5.3, which for the sake of convenience we will rewrite here

$$
H=-\sum_{j=1}^{n}\left(\frac{1+\delta}{2} \sigma_{j}^{x} \sigma_{j+1}^{x}+\frac{1-\delta}{2} \sigma_{j}^{y} \sigma_{j+1}^{y}+\frac{h}{2} \sigma_{j}^{z}\right),
$$


where $n$ is the number of spins, $\sigma_{j}^{\mu}$ are the Pauli matrices at site $j, \delta$ is the $\mathrm{x}-\mathrm{y}$ anisotropy parameter and $h$ is the strength of the magnetic field. We already pointed out that the $X Y$ model may be converted through the Jordan-Wigner transformation (25) into the quasi-free Fermion model,

$$
H=-\sum_{j=1}^{n}\left[\left(c_{j}^{\dagger} c_{j+1}+\delta c_{j}^{\dagger} c_{j+1}^{\dagger}+H . c .\right)+h\left(2 c_{j}^{\dagger} c_{j}-1\right)\right] .
$$

Ground state, and in general thermal states of quasi-free Fermion models fall within the general class of Gaussian Fermion states. We will introduce in the last chapter a general framework which allows for the derivation of the main geometric properties of such models. We will not give the details of the derivation at this stage, and we will only state the main result, which in this specific case can be derived directly. For the sake of completeness, we will consider the rotated model $H(\varphi)$ in $(24)$. Indeed from (78) and the form of the ground state (30), one gets

$$
Q_{\mu \nu}=g_{\mu \nu}+\frac{i}{2} F_{\mu \nu},
$$

where

$$
\begin{aligned}
& g_{\mu \nu}=\frac{1}{4} \sum_{k}\left(\partial_{\mu} \theta_{k} \partial_{\nu} \theta_{k}+\sin ^{2} \theta_{k} \partial_{\mu} \varphi \partial_{\nu} \varphi\right), \\
& F_{\mu \nu}=\frac{1}{2} \sum_{k}\left(\partial_{\mu} \theta_{k} \partial_{\nu} \varphi-\partial_{\nu} \theta_{k} \partial_{\mu} \varphi\right) \sin \theta_{k},
\end{aligned}
$$

with the angle $\theta_{k}$ being defined as $\theta_{k}:=\arccos \left(\eta_{k} / \varepsilon_{k}\right), \eta_{k}:=\cos q_{k}-h, \varepsilon_{k}:=\sqrt{\eta_{k}^{2}+\delta^{2} \sin ^{2} q_{k}}$, and $q_{k}=2 \pi k / n$.

One finds that the only non-vanishing derivatives are $\left(\partial_{h} \theta_{k}\right)=\delta \sin q_{k} / \varepsilon_{k}^{2},\left(\partial_{\delta} \theta_{k}\right)=\sin q_{k}\left(\cos q_{k}-h\right) / \Lambda_{v}^{2}$, and obviously $\partial_{\varphi} \varphi=1$.

In the thermodynamic limit, $g_{\mu \nu}$ can be calculated analytically by replacing the discrete variable $q_{k}$ with a continuous variable $q$ and substitute the sum with an integral, i.e., $\sum_{k=1}^{n} \rightarrow[n /(2 \pi)] \int_{0}^{\pi} \mathrm{d} q$. At critical points, this cannot be generally done, due to singular behaviour of terms involved in the sums. The resulting integrals leads to analytical expressions which differ in the two regions $|h|<1$ and $|h|>1[159,166]$,

$$
\begin{aligned}
& g_{\varphi \varphi}=\frac{n}{8}\left\{\begin{array}{cl}
\frac{|\delta|}{|\delta|+1}, & |h|<1 \\
\frac{\delta^{2}}{1-\delta^{2}}\left(\frac{|h|}{\sqrt{h^{2}-1+\delta^{2}}}-1\right), & |h|>1
\end{array}\right. \\
& g_{h h}=\frac{n}{16}\left\{\begin{array}{cl}
\frac{1}{|\delta|\left(1-h^{2}\right)}, & |h|<1 \\
\frac{|h| \delta^{2}}{\left(h^{2}-1\right)\left(h^{2}-1+\delta^{2}\right)^{3 / 2}}, & |h|>1
\end{array}\right. \\
& g_{\delta \delta}=\frac{n}{16} \begin{cases}\frac{1}{|\delta|(1+|\delta|)^{2}}, & |h|<1 \\
\left.\frac{2}{\left(1-\delta^{2}\right)^{2}}\left[\begin{array}{ll}
\frac{|h|}{\sqrt{h^{2}-1+\delta^{2}}}-1
\end{array}\right]-\frac{|h| \delta^{2}}{\left(1-\delta^{2}\right)\left(h^{2}-1+\delta^{2}\right)^{3 / 2}}\right), & |h|>1\end{cases} \\
& g_{h \delta}=\frac{n}{16}\left\{\begin{array}{cc}
0, & |h|<1 \\
\frac{-|h| \delta}{h\left(h^{2}-1+\delta^{2}\right)^{3 / 2}}, & |h|>1
\end{array} .\right.
\end{aligned}
$$

The metric as a whole shows a non-analytical behaviour across both the critical regions $|h|=1$ and $\delta=0$. To visualise more clearly such a singular behaviour, it is convenient to compute the scalar curvature, which provides a global property of the metric in each point of the phase diagram. The scalar curvature $R$, which is the trace of the Ricci curvature tensor [26], yields the following expressions

$$
\begin{array}{ll}
R & =-\frac{8}{n} \frac{1}{|\delta|} \quad|h|<1 \\
R & =\frac{8}{n}\left[4+\frac{5 h}{\sqrt{h^{2}+\delta^{2}-1}}-2 \frac{\left(h^{2}+h \sqrt{h^{2}+\delta^{2}-1}-1\right)}{\delta^{2}}\right] \quad|h|>1 .
\end{array}
$$

Note that the curvature diverges on the segment $|h| \leq 1, \delta=0$ and it is discontinuous on the lines $h= \pm 1$. Indeed, $\lim _{|h| \rightarrow 1^{+}} R=8 / n(4+3 h /|\delta|), \lim _{|h| \rightarrow 1^{-}} R=-8 / n 1 /|\delta|$.

\section{Mixed states and phase transitions}

Up to this point, this review has dealt with models at zero temperature, whose description relies on the properties of the unique ground state, i.e. a pure quantum state. A natural extension of the above considerations regards problems which 
calls for a description of the system in terms of mixed states rather than a pure quantum state. The simplest example of such a scenario would be the thermal state of a quantum system, and a natural generalisation of the above consideration would be to study phase transitions at finite temperature. As we will discuss the next few sections, the framework that we will introduce provides a way to analyse the even more general scenario of open system phase transitions, in which the properties of non-equilibrium dynamics yields mixed stable states [269-277]. To this end, we will need to bring in new tools, and we will start by introducing a mixed state generalisation of the geometric phase, i.e. the Uhlmann phase.

\subsection{Definition of mixed geometric phase via state purification}

The first definition of geometric phase for mixed state has been proposed by Uhlmann [156,278]. In his formulation a mixed state is allowed to perform any kind of physically admissible evolution. Therefore, it is truly general and applicable to the most general setting. However, admittedly, this definition relies on a rather abstract approach which somehow obscures its physical interpretation. Still, many proposals to measure it have been already put forward [186-188], and demonstrated experimentally [189,279].

The formulation of Uhlmann geometric phase relies on the concept of "purification". According to this concept, any mixed state $\rho$ can be regarded as the "reduced density matrix" of a pure state lying in an enlarged Hilbert space. Essentially, one looks for larger, possibly fictitious, quantum systems from which the original mixed states are seen as reductions of pure states. For density operators there is a standard way to do so by the use of the Hilbert-Schmidt operators, or by Hilbert-Schmidt maps, from an auxiliary Hilbert space into the original one.

\subsection{Purification}

Let us start with reviewing some basic idea of the purification procedure. Let $\mathcal{H}$ be a complex Hilbert space of finite dimension $n$ with the usual scalar product $\langle\ldots\rangle$ and let $\mathcal{B}(\mathcal{H})$ be the algebra of linear operators acting on $\mathcal{H}$. We remind that formally a general (mixed or pure) state is defined as positive linear trace class operator $\rho \in \mathcal{B}(\mathcal{H})$ such that $\operatorname{Tr} \rho=1$. In this formalism, a pure state (or rank one density operator) is any state $\omega \in \mathcal{B}(\mathcal{H})$ for which also $\omega^{2}=\omega$ holds. Using the standard notation used in Section 5.1, a pure state $\omega$ is denoted with $|\psi\rangle\langle\psi|,| \psi\rangle \in \mathcal{H}$ being the only eigenstate of $\omega$ with eigenvalue 1 .

A purification of a mixed state $\rho \in \mathcal{B}(\mathcal{H})$ is a lift to pure state $|\psi\rangle\langle\psi|$ in a larger space $\mathcal{B}\left(\mathcal{H}^{\prime}\right)$ embedding $\rho \in \mathcal{B}(\mathcal{H})$. To achieve purification, it is sufficient to consider an auxiliary Hilbert space $\mathcal{H}_{\text {aux }}$, at least of the same dimension $n$, and then consider the tensor product space:

$$
\mathcal{H} \otimes \mathcal{H}_{\text {aux }}, \quad n=\operatorname{dim} \mathcal{H} \leq \operatorname{dim} \mathcal{H}_{\text {aux }} .
$$

A reduction to $\mathcal{H}$ means performing the partial trace over the auxiliary space. Now, let $\mathbb{1}_{\text {aux }}$ be the identity operator in $\mathcal{H}_{\text {aux }}$, then a state $|\psi\rangle \in \mathcal{H} \otimes \mathcal{H}_{\text {aux }}$ is said to purify $\rho$, if for any operator $O \in \mathcal{B}(\mathcal{H})$

$$
\operatorname{Tr}(O \rho)=\left\langle\psi\left|O \otimes \mathbb{1}_{\text {aux }}\right| \psi\right\rangle,
$$

or equivalently if $\rho=\operatorname{Tr}_{\text {aux }}|\psi\rangle\langle\psi|$, where $\operatorname{Tr}_{\text {aux }}$ is the partial trace over the auxiliary space $\mathcal{H}_{\text {aux }}{ }^{2}$

However, it can be formally more convenient to work with a different notation. Indeed, being of finite dimension, $\overline{\mathcal{H}}=\mathcal{H} \otimes \mathcal{H}$ is canonically isomorphic to $\mathcal{B}(\mathcal{H})$. This can be made explicit by fixing two arbitrarily chosen orthonormal basis $\phi_{1}, \phi_{2}, \ldots$ in $\mathcal{H}$ and $\phi_{1}^{\prime}, \phi_{2}^{\prime}, \ldots$ in $\mathcal{H}_{\text {aux }}$. Given any operator $\mathbb{w} \in \mathcal{B}(\mathcal{H})$,

$$
\left|\psi_{\mathrm{w}}\right\rangle=\sum\left|\phi_{i}\right\rangle \otimes\left|\phi_{j}^{\prime}\right\rangle \cdot\left\langle\phi_{i}|\mathrm{w}| \phi_{j}^{\prime}\right\rangle \quad \in \overline{\mathcal{H}} .
$$

The canonical scalar product in $\overline{\mathcal{H}}$ is equivalent to the Hilbert-Schmidt scalar product $\left(\mathbb{w}_{1}, w_{2}\right)$ in $\mathcal{B}(\mathcal{H})$

$$
\left(\mathrm{w}_{1}, \mathrm{w}_{2}\right):=\operatorname{Tr}\left(\mathrm{w}_{1} \cdot \mathrm{w}_{2}^{\dagger}\right)=\sum\left\langle\phi_{i}\left|\mathrm{w}_{1}\right| \phi_{j}^{\prime}\right\rangle\left\langle\phi_{j}^{\prime}\left|\mathrm{w}_{2}^{\dagger}\right| \phi_{i}\right\rangle=\left\langle\psi_{\mathrm{w}_{1}} \mid \psi_{\mathrm{w}_{2}}\right\rangle,
$$

and the partial trace over the auxiliary space is given by:

$$
\begin{aligned}
\operatorname{Tr}_{a u x}(|\psi\rangle\langle\psi|) & =\sum\left|\phi_{i}\right\rangle\left\langle\phi_{k}\right| \cdot\left\langle\phi_{i}|\mathrm{w}| \phi_{j}^{\prime}\right\rangle\left\langle\phi_{j}^{\prime}\left|\mathrm{w}^{\dagger}\right| \phi_{k}\right\rangle \\
& =\mathrm{w}^{\dagger} \cdot \mathrm{w} .
\end{aligned}
$$

Therefore, given this isomorphism $\mathrm{w} \leftrightarrow \psi_{\mathrm{w}}$, in the following we will refer as standard purification or amplitude of a density matrix $\rho$ any operator $w \in \mathcal{B}(\mathcal{H})$, for which

$$
\rho=\mathrm{w}^{\dagger} \cdot \mathrm{w},
$$

or its isomorphic counterpart $\psi_{w} \in \overline{\mathcal{H}}$ defined by Eq. (101).

2 A distinguished way to choose a purification, called standard purification, is to require

$$
\mathcal{H}_{\text {aux }}=\mathcal{H}, \quad \overline{\mathcal{H}}=\mathcal{H} \otimes \mathcal{H}_{\text {aux }} .
$$

When not otherwise specified we will consider only standard purifications. 
A crucial point to stress is that, given a mixed state, the construction of a standard purification is by no means unique. From a formal point of view, it can be easily checked that any $\mathrm{w}^{\prime}=U \cdot \mathrm{w}$, for a given unitary operator $U \in U(n)$, represents a standard purification of the same state $\rho$. This is somehow expected, as the purification, from a physical point of view, represents a "complete information" on the global system described by the global Hilbert space $\mathcal{H} \otimes \mathcal{H}_{\text {aux }}$, whereas $\rho$ describes only a part of this compound. Therefore, $\rho$ is expected to contain only that part of the "information" which can be "locally" stored in one of the subsystem $\mathcal{H}$. This becomes physically obvious by considering that the transformation $U$ in $\overline{\mathcal{H}}=\mathcal{H} \otimes \mathcal{H}_{\text {aux }}$ looks just like a local change of basis in $\mathcal{H}_{a u x}$, which by no means can affect the state $\rho$ in $\mathcal{H}$.

In the next section, we will often stress the implications of this "one to many" relation between mixed states and their purifications. Indeed, for what concerns the definition of mixed state geometric phase it will become crucial to establish a criterion which diminish such an ambiguity, by selecting distinguished set of purifications.

\subsection{Parallel transport of density matrices and Uhlmann geometric phase}

Given this definition of purification, it would be natural to generalise the concept of geometric phase for a chain of density matrices, by referring to their purifications. Indeed, as purifications are, by definition, pure, we could just straightforwardly apply the definition of Berry phases exploited in the previous sections. Unfortunately, this programme does not generate an unambiguous value of the geometric phase, on account of the lack of uniqueness in the purification procedure. The problem, is, therefore, to select among all possible ones a distinguished set of purification. A solution to this problem was proposed by Uhlmann [17,156,280,281]. His idea is based on the concept of parallel transport.

Let us start by considering a path of density operator $\rho(s), s \in\left[s_{a}, s_{b}\right]$, and its purified path

$$
\rho(s) \rightarrow \mathbb{w}(s),
$$

i.e. such that $\rho(s)=\mathrm{w}^{\dagger}(s) \cdot \mathrm{w}(s)$. By the previous argument, not only (106) represents a purification but also every unitarily transformed path

$$
\mathrm{w}(s) \rightarrow U(s) \cdot \mathrm{w}(s) .
$$

By analogy with the idea of gauge transformation used in section for pure states, it is natural to refer to (107) as a gauge transformation for mixed states, and in general the freedom in the choice of an amplitude w as gauge freedom. Notice, that the set of possible gauge transformations that could be adopted in the case of the pure states were mere multiplications by complex phase factor's $e^{i \alpha} \in U(1)$, i.e. a $U(1)$ gauge freedom. This allowed for the description of the Berry phase in terms of an underlying Abelian gauge theory, which by analogy could be compared with the usual electromagnetic $U(1)$ gauge field. The much wider choice of a general unitary operator $U \in U(n)$, in the present case, calls for the more convoluted $U(n)$ gauge structure. We will show, that the natural setting underlying the definition of the Uhlmann geometric phase is within the theory of holonomies, i.e. the non-Abelian generalisation of the geometric phase.

Let $\mathbb{w}_{1}, w_{2}, \ldots, \mathbb{w}_{m}$ be a finite subdivision of the curve (106), i.e. a path ordered subset of operators (106). Notice that these operators have norm $\left\|\mathrm{w}_{i}\right\|^{2}:=\left(\mathrm{w}_{i}, \mathrm{w}_{i}\right)=1$, due to the normalisation condition of $\rho_{i}$. Let us consider the discrete chain of pure states $\psi_{\mathrm{w}_{i}}$

$$
\begin{aligned}
& \xi=\left(\mathbb{w}_{1}, \mathbb{w}_{2}\right)\left(\mathbb{w}_{2}, \mathbb{w}_{3}\right) \ldots\left(\mathbb{w}_{m-1}, w_{m}\right)\left(\mathbb{w}_{m}, \mathbb{w}_{1}\right) \\
& =\left\langle\psi_{\mathrm{w}_{1}} \mid \psi_{\mathrm{w}_{2}}\right\rangle\left\langle\psi_{\mathrm{w}_{1}} \mid \psi_{\mathrm{w}_{3}}\right\rangle \ldots\left\langle\psi_{\mathrm{w}_{m-1}} \mid \psi_{\mathrm{w}_{m}}\right\rangle\left\langle\psi_{\mathrm{w}_{m}} \mid \psi_{\mathrm{w}_{1}}\right\rangle .
\end{aligned}
$$

One could be tempted to consider the complex argument of the functional $\xi$, and take the limit from the discrete chain $\mathrm{w}_{1} \ldots \mathrm{w}_{n}$ to a continuous evolution, and identify this limit with the "mixed state geometric phase" of the path $\rho(s)$. However, each gauged transformed path (107) generally produces a different $\tilde{\xi} \neq \xi$. A sensible criterion to diminish this arbitrariness is needed.

Uhlmann introduced a parallel transport criterion, analogous to the parallel transport condition for pure states, which is able to single out a specific set of purified paths and uniquely identifies the geometric phase. In fact, if one tries to purify two density operators, $\rho_{1}$ and $\rho_{2}$, simultaneously, say with $\psi_{\mathrm{w}_{1}}$ and $\psi_{\mathrm{w}_{2}}$, the purification ambiguity can be partially lifted by choosing them to be "as near as possible" to each other [17,18,25,282]. Given $\left|\psi_{\mathrm{w}_{1}}\right\rangle$, there is a $\left|\psi_{\mathrm{w}_{2}}\right\rangle$ with maximal overlap

$$
\left|\left\langle\psi_{\mathrm{w}_{1}} \mid \psi_{\mathrm{w}_{2}}\right\rangle\right| \geq\left|\left\langle\psi_{\mathrm{w}_{1}}^{\prime} \mid \psi_{\mathrm{w}_{2}}^{\prime}\right\rangle\right|
$$

or, equivalently, with minimal Fubini-Study distance $d_{F S}^{2}\left(\psi_{\mathrm{w}_{1}}, \psi_{\mathrm{w}_{2}}\right)=2-2\left|\left\langle\psi_{\mathrm{w}_{1}} \mid \psi_{\mathrm{w}_{2}}\right\rangle\right|$ among all pair of vectors, $\left|\psi_{\mathrm{w}_{1}}^{\prime}\right\rangle$, $\left|\psi_{\mathrm{w}_{2}}^{\prime}\right\rangle$ simultaneously purifying $\rho_{1}$ and $\rho_{2}$. Uhlmann describes this situation by calling the pair $\psi_{\mathrm{w}_{1}}$ and $\psi_{\mathrm{w}_{2}}$ parallel, as a shorthand for "as parallel as possible" [156]. This criterion, therefore, allows one to distinguish, within all purifications $\mathrm{w}_{i}$ of the curve $\rho(s)$, the exceptional ones $\tilde{\mathrm{w}}_{i}$ for which the overlap between an element of the purified chain $\tilde{\mathrm{w}}_{i}$ and the neighbouring one $\tilde{w}_{i+1}$ is maximised. If this condition is fulfilled for the whole chain, the remaining arbitrariness is in a regauging $\tilde{\mathrm{w}}_{i} \rightarrow e^{i \alpha_{i}} U \cdot \tilde{\mathrm{w}}_{i}$ of the subdivision, with $\alpha_{i} \in \mathbb{R}$ and a unitary operator $U$ independent of the index $i$. This, 
however, leaves the quantity

$$
\tilde{\xi}=\left(\tilde{\mathbb{w}}_{1}, \tilde{\mathbb{w}}_{2}\right)\left(\tilde{\mathbf{w}}_{2}, \tilde{\mathrm{w}}_{3}\right) \ldots\left(\tilde{\mathbb{w}}_{m-1}, \tilde{\mathrm{w}}_{m}\right)\left(\tilde{\mathbb{w}}_{m}, \tilde{\mathrm{w}}_{1}\right)
$$

invariant. Therefore, $\tilde{\xi}$ is uniquely defined by the discrete chain of state $\rho_{i}=\mathrm{w}_{i}^{\dagger} \mathrm{w}_{i}$ and it is meaningful to regard $\Phi_{g}=\arg \tilde{\xi}$ as a mixed state generalisation of the geometric phase.

One can also sharpen the condition of parallel transport (110) by making use of the remaining regauging degree of freedom. One can, indeed, require two neighbouring purifications to be in phase, in the Pancharatnam criterion, i.e.

$$
\left(\tilde{w}_{i}, \tilde{w}_{i+1}\right)=\left\langle\psi_{w_{i}} \mid \psi_{w_{i+1}}\right\rangle \geq 0 .
$$

For such a parallel purification, the mixed geometric phase becomes:

$$
\Phi_{g}=\arg \left(\tilde{\mathrm{w}}_{N}, \tilde{\mathrm{w}}_{1}\right) \text {. }
$$

The condition (112) is equivalent to requiring that

$$
\left\|\psi_{\mathrm{w}_{1}}-\psi_{\mathrm{w}_{2}}\right\|+\left\|\psi_{\mathrm{w}_{2}}-\psi_{\mathrm{w}_{3}}\right\|+\cdots+\left\|\psi_{\mathrm{w}_{N-1}}-\psi_{\mathrm{w}_{N}}\right\|
$$

attains its minimum. Going to the limit of finer and finer subdivisions, Eq. (114) converges to the length of the curve of the purification (106). Therefore the purification is parallel if and only if it solves the following variational problem,

$$
D_{B}=\int_{\rho\left(s_{a}\right)}^{\rho\left(s_{b}\right)} d l_{B}:=\min \int_{s_{a}}^{s_{b}} \sqrt{\left\langle\dot{\psi}_{\mathrm{w}(s)} \mid \dot{\psi}_{\mathrm{w}(s)}\right\rangle} d s,
$$

where $\psi_{\mathrm{w}(s)}$ is a purified path of $\rho(s)$, and the dots denote derivatives with respect to $s$. The resulting minimal length $D_{B}$ is called Bures length or Bures angle [25,283]. Therefore a purification $\psi_{\mathrm{w}(s)}$ is called "parallel" or "parallel transported" if, for every gauged purification $\psi_{\mathrm{w}(s)}^{\prime}$ of $\rho(s)$, it holds

$$
\sqrt{\left\langle\dot{\psi}_{\mathrm{w}(s)} \mid \dot{\psi}_{\mathrm{w}(s)}\right\rangle} \leq \sqrt{\left\langle\dot{\psi}_{\mathrm{w}(s)}^{\prime} \mid \dot{\psi}_{\mathrm{w}(s)}^{\prime}\right\rangle} \quad \forall s .
$$

It is plain to derive a condition for parallel purification, which is easier to handle. Suppose that $\psi_{\mathrm{w}(s)}$ is a parallel purification, then $\psi_{\mathrm{w}(s)}^{\prime}=U(s) \psi_{\mathrm{w}(s)}$, with $U(s)=\mathbb{1} \otimes U^{\prime}$ unitary, is another purification of $\rho(s)$. Inserting this into Eq. (116) leads to

$$
0 \leq\left\langle\psi_{\mathrm{w}}\left|B^{\dagger} B\right| \psi_{\mathrm{w}}\right\rangle+i\left(\left\langle\dot{\psi}_{\mathrm{w}}|B| \psi_{\mathrm{w}}\right\rangle-\left\langle\psi_{\mathrm{w}}|B| \dot{\psi}_{\mathrm{w}}\right\rangle\right),
$$

where $B$ is the Hermitian generator of $U$, i.e. $B(s):=i \dot{U}(s) U^{\dagger}(s)$. This inequality is valid if and only if $\left\langle\dot{\psi}_{\mathrm{w}}|B| \psi_{\mathrm{w}}\right\rangle=$ $\left\langle\psi_{\mathrm{w}}|B| \dot{\psi}_{\mathrm{w}}\right\rangle$ for all Hermitian operators $B=\mathbb{1} \otimes B^{\prime}$. In the language of the Hilbert-Schmidt space, this condition becomes $\operatorname{Tr}\left(\dot{w} w^{\dagger} B^{\prime}-w_{w} \dot{w}^{\dagger} B^{\prime}\right)=0$ for all $B^{\prime}$ Hermitian, which essentially means nothing other than

$$
\dot{w}_{\mathbb{w}}^{\dagger}=\mathbb{w} \dot{w}^{\dagger} .
$$

This condition, together with the normalisation of $\rho(s)$, implies that $(\mathbb{w}(s), w(s+\delta s)) \approx 1$, thus guaranteeing that each $\mathrm{w}(s)$ and its neighbour $\mathrm{w}(s+\delta s)$ are in phase in the Pancharatnam sense. The Uhlmann mixed geometric phase results just in the residual phase difference between initial and final state, i.e.

$$
\Phi_{g}=\arg \left(\mathbb{w}\left(s_{b}\right), w\left(s_{a}\right)\right),
$$

with $s_{a}$ and $s_{b}$ initial and final value of the parameter, respectively.

\section{Fidelity and Bures metric}

According to the Uhlmann parallelism, two amplitudes $\mathrm{w}_{1}$ and $\mathrm{w}_{2}$ are called parallel if they maximise their HilbertSchmidt scalar product, among those simultaneously purifying $\rho_{1}$ and $\rho_{2}$. A very important byproduct of this maximisation procedure is the so called fidelity [17], defined as

$$
\mathcal{F}\left(\rho_{1}, \rho_{2}\right):=\max _{\mathrm{w}_{1}, \mathrm{w}_{2}}\left|\left(\mathrm{w}_{1}, \mathrm{w}_{2}\right)\right|=\max _{\psi_{\mathrm{w}_{1}}, \psi_{\mathrm{w}_{2}}}\left|\left\langle\psi_{\mathrm{w}_{1}} \mid \psi_{\mathrm{w}_{2}}\right\rangle\right| .
$$

This is a very crucial quantity in quantum information and in quantum estimation theory. It provides an operationally well-defined distance between quantum states, in terms of statistical distinguishability of quantum states. An explicit expression for the above maximal value has been proven by Uhlmann $[17,22]$

$$
\mathcal{F}\left(\rho_{1}, \rho_{2}\right)=\operatorname{Tr} \sqrt{\sqrt{\rho_{2}} \rho_{1} \sqrt{\rho_{2}}},
$$

which readily shows how the fidelity depends on $\rho_{1}$ and $\rho_{2}$ only. The proof of the above expression relies on the following simple lemma. 
Lemma 1. For any operator $B$, and any unitary $U,|\operatorname{Tr}(B U)| \leq \operatorname{Tr}|B|$, with equality attained for $U=V^{\dagger}$, where $B=:|B| V$ is the polar decomposition of $B$, with $|B|:=\sqrt{B B^{\dagger}}$.

Proof. The equality follows straightforwardly from the condition stated, whereas the inequality arises from

$$
|\operatorname{Tr} B U|=|\operatorname{Tr}(|B| V U)|=\left|\operatorname{Tr}\left(|B|^{\frac{1}{2}}|B|^{\frac{1}{2}} V U\right)\right| \leq \sqrt{\operatorname{Tr}|B| \operatorname{Tr}\left(U^{\dagger} V^{\dagger}|B| V U\right)}=\operatorname{Tr}|B|,
$$

where the second relation is the Cauchy-Schwarz inequality for the Hilbert-Schmidt scalar product.

To prove Eq. (121), we define $\mathrm{w}_{i}=: \sqrt{\rho_{i}} U_{i}$, with $i=(1,2)$ the polar decompositions of two purifications of $\rho_{1}$ and $\rho_{2}$. Then, by Lemma 1

$$
\left|\operatorname{Tr}\left(\mathrm{w}_{1}^{\dagger} \mathrm{w}_{2}\right)\right|=\left|\operatorname{Tr}\left(\sqrt{\rho_{1}} \sqrt{\rho_{2}} U_{2} U_{1}^{\dagger}\right)\right| \leq \operatorname{Tr}\left|\sqrt{\rho_{1}} \sqrt{\rho_{2}}\right|=\operatorname{Tr} \sqrt{\sqrt{\rho_{1}} \rho_{2} \sqrt{\rho_{1}}} .
$$

The equality is attained for $U_{2} U_{1}^{\dagger}=V^{\dagger}$, where $\sqrt{\rho_{1}} \sqrt{\rho_{2}}=:\left|\sqrt{\rho_{1}} \sqrt{\rho_{2}}\right| V$.

Two important limiting case of the fidelity are worth mentioning explicitly. The first one is when we deal with pure states, $\rho_{1}=\left|\psi_{1}\right\rangle\left\langle\psi_{1}\right|$ and $\rho_{2}=\left|\psi_{2}\right\rangle\left\langle\psi_{2}\right|$. In this case, the fidelity reduces to the standard overlap $\mathcal{F}\left(\rho_{1}, \rho_{2}\right)=\left|\left\langle\psi_{1} \mid \psi_{2}\right\rangle\right|$. Slightly more generally, if just one of the two states is pure, $\rho_{1}=\left|\psi_{1}\right\rangle\left\langle\psi_{1}\right|$, then $\mathcal{F}\left(\rho_{1}, \rho_{2}\right)^{2}=\left|\left\langle\psi_{1}\left|\rho_{2}\right| \psi_{1}\right\rangle\right|$, which is the probability that the state $\rho_{2}$ will score positively if tested on whether it is in the pure state $\rho_{1}$. It serves as a figure of merit in many statistical estimation problems.

The second example is when $\rho_{1}$ and $\rho_{2}$ commute, i.e. when they are simultaneously diagonal, $\rho_{i}=\sum_{k} p_{i}(k)|k\rangle\langle k|$. In this case, the fidelity reduces to

$$
\mathcal{F}\left(\rho_{1}, \rho_{2}\right)=\sum_{k} \sqrt{p_{1}(k)} \sqrt{p_{2}(k)}=B\left(P_{1}, P_{2}\right),
$$

i.e. the Bhattacharyya coefficient of the classical statistical distributions $P_{i}:=\left\{p_{i}(k), k=1 \ldots n\right\}$.

The fidelity also enjoys a number of quite desirable properties for a measure of statistical distinguishability [22]:

1. $0 \leq \mathcal{F}\left(\rho_{1}, \rho_{2}\right) \leq 1$;

2. $\mathcal{F}\left(\rho_{1}, \rho_{2}\right)=1$ iff $\rho_{1}=\rho_{2}$ and $\mathcal{F}\left(\rho_{1}, \rho_{2}\right)=0$ iff $\rho_{1}$ and $\rho_{2}$ have orthogonal supports;

3. Symmetry, $\mathcal{F}\left(\rho_{1}, \rho_{2}\right)=\mathcal{F}\left(\rho_{2}, \rho_{1}\right)$;

4. Strong concavity, $\mathcal{F}\left(\sum_{j} p_{j} \rho_{j}, \sum_{j} q_{j} \rho_{j}^{\prime}\right) \geq \sum_{j} \sqrt{p_{j} q_{j}} \mathcal{F}\left(\rho_{j}, \rho_{j}^{\prime}\right)$;

5. Multiplicativity, $\mathcal{F}\left(\rho_{1} \otimes \rho_{2}, \rho_{3} \otimes \rho_{4}\right)=\mathcal{F}\left(\rho_{1}, \rho_{3}\right) \mathcal{F}\left(\rho_{2}, \rho_{4}\right)$;

6. Unitary invariance, $\mathcal{F}\left(\rho_{1}, \rho_{2}\right)=\mathcal{F}\left(U \rho_{1} U^{\dagger}, U \rho_{2} U^{\dagger}\right)$;

7. Monotonicity, $\mathcal{F}\left(\Phi\left(\rho_{1}\right), \Phi\left(\rho_{2}\right)\right) \leq \mathcal{F}\left(\rho_{1}, \rho_{2}\right)$, where $\Phi$ is a trace preserving CP map.

Property 4 also implies concavity, i.e. $\mathcal{F}\left(\sum_{j} p_{j} \rho_{j}, \rho^{\prime}\right) \geq \sum_{j} \sqrt{p_{j} q_{j}} \mathcal{F}\left(\rho_{j}, \rho^{\prime}\right)$. Property 7 is a key entry, it means that the fidelity cannot grow under any type of physical process, i.e. unitary transformations, generalised measurements, stochastic maps and combinations thereof. This is a crucial demand for any bona-fide measure of distinguishability. It is, indeed, physically unacceptable that any stochastic map, including measurements, may contribute in increasing the distinguishability of two states.

To explicitly see in what sense the fidelity is a measure of statistical distinguishability [284] let us follow a similar argument exposed in Section 6.2. In the case of two pure states $\psi_{1}$ and $\psi_{2}$, it has been shown that the overlap $\left|\left\langle\psi_{1} \mid \psi_{2}\right\rangle\right|$ provides a measure of the experimental (in)-distinguishability of two quantum states, assuming no limitations on the type of measurements one can perform. One can show that the same applies to the fidelity in the case of two mixed states $\rho_{1}$ and $\rho_{2}$. One assumes a specific measurement process, and defines a statistical measure of distinguishability between the two resulting outcome distributions. This measure clearly depends on the choice of the measurement process as well as on the states. One can then select the optimal measurement strategy that maximises the distinguishability according to some figure of merit, and define the latter as the measure of distinguishability between the states.

For simplicity we will assume both states to be full-rank (i.e. invertible) density matrices. We will allow for the most general type of measuring device, i.e. a positive operator valued measurement (POVM) [285] $\left\{E_{k}, k=1 \ldots n\right\}$. A given density matrix $\rho_{i}$ responds to such a measurement process with a probability distribution $P_{i}:=\left\{p\left(\rho_{i}, E_{k}\right), k=1 \ldots n\right\}$, where $p_{i}(k):=\operatorname{Tr}\left(\rho_{i} E_{k}\right)$. The optimal POVM is the one that produces two distributions $P_{1}$ and $P_{2}$ which are the most statistically distinguishable. As in the case of pure states, the figure of merit of choice is the Bhattacharyya coefficient

$$
B\left(P_{1}, P_{2}\right)=\sum_{k} \sqrt{p_{1}(k) p_{2}(k)},
$$

which has to be minimised over the POVMs. For a generic unitary operator $U$, rewriting

$$
p_{1}(k):=\operatorname{Tr}\left(\left(U \sqrt{\rho_{1}} \sqrt{E_{k}}\right)\left(U \sqrt{\rho_{1}} \sqrt{E_{k}}\right)^{\dagger}\right)
$$


yields the following chain of relations,

$$
\begin{aligned}
B\left(P_{1}, P_{2}\right) & =\sum_{k} \operatorname{Tr}\left(\left(U \sqrt{\rho_{1}} \sqrt{E_{k}}\right)\left(U \sqrt{\rho_{1}} \sqrt{E_{k}}\right)^{\dagger}\right)^{\frac{1}{2}} \operatorname{Tr}\left(\left(\sqrt{\rho_{2}} \sqrt{E_{k}}\right)\left(\sqrt{\rho_{2}} \sqrt{E_{k}}\right)^{\dagger}\right)^{\frac{1}{2}} \\
& \geq \sum_{k}\left|\operatorname{Tr}\left(\left(U \sqrt{\rho_{1}} \sqrt{E_{k}}\right)\left(\sqrt{\rho_{2}} \sqrt{E_{k}}\right)^{\dagger}\right)\right|=\sum_{k}\left|\operatorname{Tr}\left(U \sqrt{\rho_{1}} E_{k} \sqrt{\rho_{2}}\right)\right| \\
& \geq\left|\operatorname{Tr}\left(\sum_{k} U \sqrt{\rho_{1}} E_{k} \sqrt{\rho_{2}}\right)\right|=\left|\operatorname{Tr}\left(U \sqrt{\rho_{1}} \sqrt{\rho_{2}}\right)\right|,
\end{aligned}
$$

where the second line is due to the Cauchy-Schwarz inequality for the Hilbert-Schmidt scalar product, with the equality being attained if condition (a): $\sqrt{\rho_{2}} E_{k} \propto U \sqrt{\rho_{1}} E_{k}$ is fulfilled. The remaining relations arise from the linearity of the trace and completeness property of the POVMs, and the second inequality can be saturated only if (b): $\operatorname{Tr}\left(U \sqrt{\rho_{1}} E_{k} \sqrt{\rho_{2}}\right) \geq 0$ $\forall k$.

Notice that the above inequalities are fulfilled by any unitary $U$. Therefore, if it has to be a chance of attaining equality in them, $U$ had better be chosen so as to maximise $\left|\operatorname{Tr}\left(U \sqrt{\rho_{1}} \sqrt{\rho_{2}}\right)\right|$. From Lemma 1, we know that this is achieved by

$$
U=\sqrt{\sqrt{\rho_{2}} \rho_{1} \sqrt{\rho_{2}}} \rho_{2}^{-\frac{1}{2}} \rho_{1}^{-\frac{1}{2}} \text {. }
$$

It can be checked that, with this unitary operator both condition (a) and (b) can be satisfied by a set of POVMs $E_{k}$, which are projective measurements onto the eigenbasis of the following Hermitian operator

$$
M:=\rho_{2}^{-\frac{1}{2}} \sqrt{\sqrt{\rho_{2}} \rho_{1} \sqrt{\rho_{2}}} \rho_{2}^{-\frac{1}{2}} .
$$

The end result is $B\left(P_{1}, P_{2}\right)=\mathcal{F}\left(\rho_{1}, \rho_{2}\right)$.

This finally establishes the interpretation of the fidelity as a statistical measure of distinguishability. This parallels the discussion we made regarding the Fubini-Study metric in Section 6.2, when the states to be distinguished were pure, i.e. $\rho_{i}=\left|\psi_{i}\right\rangle\left\langle\psi_{i}\right|$. In that case, it was found that the Bhattacharyya coefficient distinguishing the probability distributions for of the optimal measurement apparatus equalled the overlap $\left|\left\langle\psi_{1} \mid \psi_{2}\right\rangle\right|$. These two solutions are consistent. However, while for pure states several optimal measurements are possible, here the observable $M$ providing the optimal distinguishability is uniquely defined. We have derived its expression, and it corresponds to the geometric mean of the operators $\rho_{2}^{-1}$ and $\rho_{1}$.

\subsection{The Bures metric}

The fidelity provides a natural way of defining a distance on the space of density matrices. The definition (120) of the fidelity is based on a suitably optimised overlap between pure states. We could therefore borrow the considerations on the Fubini-Study metric exposed in Section 6.2, and apply them verbatim to purifications. Once the optimisation over the purification is carried out, the result is the definition of two Riemannian distances, called Bures distance

$$
d_{B}\left(\rho_{1}, \rho_{2}\right):=\sqrt{2-2 \mathcal{F}\left(\rho_{1}, \rho_{2}\right)} ;
$$

and Bures length or Bures angle

$$
D_{B}\left(\rho_{1}, \rho_{2}\right):=\arccos \mathcal{F}\left(\rho_{1}, \rho_{2}\right) .
$$

Clearly, these are the generalisations of the Fubini-Study distance $d_{F S}$ and Fubini-Study length $D_{F S}$, respectively, when the states $\rho_{1}$ and $\rho_{2}$ are allowed to be mixed. Like in the case of the Fubini-Study metric, the first distance $d_{B}$ measures the length of a straight chord, while $D_{B}$ measures the length of a curve within the manifold of density matrices. By construction, they are Riemannian distances, and are consistent with the same Riemannian metric. Moreover, notice that they are both monotonously decreasing functions of the fidelity. This means that $d_{B}$ and $D_{B}$ can be regarded as distances that measure the statistical distinguishability between two quantum states. This is further confirmed by the monotonicity property 7 , which entails that both $d_{B}$ and $D_{B}$ are non-decreasing under stochastic maps, i.e. under any physically meaningful quantum operations.

With the confidence that we are investigating a relevant definition of distance, let us turn to the Riemannian metric induced by the Bures distance, or equivalently by the Bures length. In the limit of two density matrices infinitesimally apart $\rho(s)$ and $\rho(s+d s)$, both $d_{B}$ and $D_{B}$ converge to the infinitesimal length

$$
d l_{B}^{2}:=\min \operatorname{Tr}\left(\dot{\mathrm{w}}^{\dagger} \dot{\mathrm{w}}\right) d s^{2},
$$

in agreement with the expression (115), where the minimum is attained by the amplitudes w( $s$ ) fulfilling the parallel transport condition (118). This is the Bures metric. It is easy to check that condition (118) is fulfilled by the following ansatz $[280,286,287]$

$$
\dot{\mathrm{w}}=G \mathbb{w}, \quad G^{\dagger}=G .
$$


$G$ can be determined by differentiating $\rho=w_{w}{ }^{\dagger}$ and inserting (129), which yields:

$$
\dot{\rho}=G \rho+\rho G .
$$

The quantity G, which may be called the parallel transport generator (PTG), is implicitly defined as the (unique) operator solution of (130) with the auxiliary requirement that

$$
\langle\psi|G| \psi\rangle=0 \text {, whenever } \rho|\psi\rangle=0 .
$$

In terms of $G$, the Bures metric can be cast in the following forms

$$
d l_{B}^{2}:=\operatorname{Tr}\left(\mathrm{w}^{\dagger} G^{2} \mathrm{w}\right) d s^{2}=\operatorname{Tr}\left(G^{2} \rho\right) d s^{2}=\frac{1}{2} \operatorname{Tr}(G \dot{\rho}) d s^{2},
$$

where $g_{\mu \nu}$ is the Bures metric tensor. Assume that the $\rho(\lambda)$ is a collection of density matrices labelled by a set of parameters $\lambda:=\left\{\lambda_{\mu}\right\}$ belonging to a manifold $\mathcal{M}$. The components of the Bures metric on the induced manifold are given by

$$
d l_{B}^{2}=: \sum_{\mu \nu} g_{\mu \nu} d \lambda_{\mu} d \lambda_{\nu}, \quad g_{\mu \nu}=\frac{1}{2} \operatorname{Tr}\left(\left\{G_{\mu}, G_{\nu}\right\} \rho\right),
$$

where $\{.,$.$\} is the anti-commutator, and G_{\mu}$ is the restriction of $G$ along the coordinate $\lambda_{\mu}$, and it is determined by the analog of Eq. (130), i.e. $\partial_{\mu} \rho=G_{\mu} \rho+\rho G_{\mu}$. We can also raise $G$ to the rank of an operator-valued differential one-form, defined as $\boldsymbol{G}:=\sum_{\mu} G_{\mu} d \lambda_{\mu}$, which clearly obeys

$$
d \rho=\boldsymbol{G} \rho+\rho \boldsymbol{G} .
$$

This expression can be easily solved in the basis of eigenvalues of the density matrix $\rho=\sum_{k} p_{k}|k\rangle\langle k|$

$$
\langle j|\boldsymbol{G}| k\rangle=\sum_{p_{j}>0, p_{k}>0} \frac{\langle j|d \rho| k\rangle}{p_{j}+p_{k}},
$$

where the restriction $p_{j}>0, p_{k}>0$ on the summation derives from the auxiliary condition (131). Casting this expression into Eq. (133) leads to the following explicit form for the Bures metric tensor [288,289],

$$
g_{\mu \nu}=\frac{1}{2} \sum_{p_{j}>0, p_{k}>0} \frac{\left\langle j\left|\partial_{\mu} \rho\right| k\right\rangle\left\langle k\left|\partial_{\nu} \rho\right| j\right\rangle}{p_{j}+p_{k}} .
$$

Now, let us cast the expression (135) in a form amenable to interesting considerations. Let us first differentiate the density matrix $\partial_{\mu} \rho=\sum_{k}\left(\partial_{\mu} p_{k}|k\rangle\left\langle k\left|+p_{k}\right| \partial_{\mu} k\right\rangle\left\langle k\left|+p_{k}\right| k\right\rangle\left\langle\partial_{\mu} k\right|\right)$ and consider the matrix element $\left(\partial_{\mu} \rho\right)_{\mu \nu}$. Notice that $\langle j \mid k\rangle=\delta_{j, k} \Rightarrow\left\langle\partial_{\mu} j \mid k\right\rangle=-\left\langle i \mid \partial_{\mu} k\right\rangle$; whence $\left\langle j\left|\partial_{\mu} \rho\right| k\right\rangle=\delta_{j, k} \partial_{\mu} p_{j}+\left\langle j \mid \partial_{\mu} k\right\rangle\left(p_{k}-p_{j}\right)$. Plugging this expression back into (135) yields

$$
g_{\mu \nu}=\frac{1}{4} \sum_{p_{k}>0} p_{k}\left(\frac{\partial_{\mu} p_{k}}{p_{k}}\right)\left(\frac{\partial_{\mu} p_{k}}{p_{k}}\right)+\frac{1}{2} \sum_{\substack{p_{j}>0 \\ p_{k}>0}}\left\langle j \mid \partial_{\mu} k\right\rangle\left\langle\partial_{\nu} k \mid j\right\rangle \frac{\left(p_{j}-p_{k}\right)^{2}}{p_{j}+p_{k}} .
$$

This expression provides an interesting distinction between a classical and a quantum contribution. Indeed, the first term in (136) is the so called Fisher-Rao metric. This is the metric induced by both the Hellinger distance and the Bhattacharyya distance between the infinitesimally close probability distributions $\left\{p_{k}\right\}$ and $\left\{p_{k}+d p_{k}\right\}$. The second term takes into account the generic non-commutativity of $\rho$ and $\rho^{\prime}:=\rho+d \rho$. Thus, one may refer to these two terms as the classical and non-classical contributions to the metric, respectively. When $\left[\rho^{\prime}, \rho\right]=0$ the problem reduces to an effective classical problem and the Bures metric obviously collapses into the Fisher-Rao metric.

One can draw an interesting connection between the metric (135) and a quantity of quantum information known as quantum Chernoff bound [290]. This is the generalisation of the classical Chernoff bound used in information theory [291,292]. Consider an experimental procedure aiming at distinguishing two quantum states $\rho_{1}$ and $\rho_{2}$, where a large number $n$ of copies are provided, and collective measurement are allowed for. In the limit of very large $n$, the probability of error in discriminating $\rho_{1}$ and $\rho_{2}$ decays exponentially as $P_{\text {err }} \sim \exp \left(-n \xi_{Q C B}\right)$, where $\xi_{Q C B}$ denotes the quantum Chernoff bound. The Chernoff bound generates a metric over the space of quantum states naturally endowed with an operationally well defined character: The farther apart two states lie according to this distance, the smaller is the asymptotic error rate of a procedure that attempts to tell them apart.

In [290] it has been proved that

$$
\exp \left(-\xi_{Q С B}\right)=\min _{0 \leq s \leq 1} \operatorname{tr}\left(\rho_{1}^{s} \rho_{2}^{1-s}\right) \leq \mathcal{F}\left(\rho_{1}, \rho_{2}\right),
$$

which, for infinitesimally close states $\rho_{1}=\rho$ and $\rho_{2}=\rho+d \rho$, yields

$$
d l_{Q C B}^{2}:=1-\exp \left(-\xi_{Q C B}\right)=\sum_{\mu \nu} g_{\mu \nu}^{Q C B} d \lambda_{\mu} d \lambda_{\nu},
$$


where

$$
g_{\mu \nu}^{Q C B}=\frac{1}{2} \sum_{j, k} \frac{\left\langle j\left|\partial_{\mu} \rho\right| k\right\rangle\left\langle k\left|\partial_{\nu} \rho\right| j\right\rangle}{\left(\sqrt{p_{j}}+\sqrt{p_{k}}\right)^{2}} .
$$

This expression shows both distinguishability distances, the quantum Chernoff bound metric and the Bures metric (135), share similar structures. They are identical except for the denominators, where $p_{j}+p_{k}$ are replaced by $\left(\sqrt{p_{j}}+\sqrt{p_{k}}\right)^{2}$. The following inequalities $\left(\sqrt{p_{m}}+\sqrt{p_{n}}\right)^{2} \geq p_{n}+p_{m}$ and $2\left(p_{j}+p_{k}\right) \geq\left(\sqrt{p_{j}}+\sqrt{p_{k}}\right)^{2}$ imply the equivalence of these two metric tensors, i.e.

$$
\frac{g_{\mu \nu}}{2} \leq g_{\mu \nu}^{Q C B} \leq g_{\mu \nu}
$$

Therefore one expects the two distinguishability measures to convey equivalent information as far as local properties of the manifold of quantum states are concerned.

\section{Uhlmann connection and Uhlmann curvature}

In the closed path $\rho_{\lambda(s)}$, initial and final amplitudes are related by a unitary transformation, i.e. $\mathbb{w}_{\lambda\left(s_{b}\right)}=\mathbb{w}_{\lambda\left(s_{a}\right)} V_{\gamma}$. If the path of amplitudes $\mathrm{w}_{\lambda(s)}$ fulfils the Uhlmann condition, $V_{\gamma}$ is a holonomy, i.e. the non-Abelian generalisation of Berry phase [156]. The holonomy is expressed as

$$
V_{\gamma}=\mathcal{P} e^{i \oint_{\gamma} A},
$$

where $\mathcal{P}$ is the path ordering operator and $\boldsymbol{A}$ is the Uhlmann connection one-form. The Uhlmann connection can be derived from the following ansatz [278,280]

$$
d_{\mathbb{W}}=i_{\mathbb{W}} \boldsymbol{A}+\boldsymbol{G}_{\mathbb{W}},
$$

which is the generalisation of (129) when the parallel transport condition is lifted. By differentiating $\rho=w_{w}{ }^{\dagger}$ and using the defining property of $\boldsymbol{G}$ (see Eq. (130)), it follows that $\boldsymbol{A}$ is Hermitian and it is implicitly defined by the equation

$$
\boldsymbol{A}_{\mathbb{w}}^{\dagger} \mathbb{w}+\mathbb{w}^{\dagger} \mathbb{w} \boldsymbol{A}=i\left(d_{\mathbb{w}}^{\dagger} \mathbb{w}^{\dagger}-\mathbb{w}^{\dagger} d_{\mathbb{w}}\right),
$$

with the auxiliary constraint that $\left\langle\psi^{\prime}|A| \psi^{\prime}\right\rangle=0$, for $w\left|\psi^{\prime}\right\rangle=0$. From Eq. (142), it can be checked that $\boldsymbol{A}$ obeys the expected transformation rule of non-Abelian gauge potentials,

$$
\boldsymbol{A} \rightarrow U^{\dagger}(s) \boldsymbol{A} U(s)+i U^{\dagger}(s) d U(s), \quad \text { under } \quad \mathbb{w}(s) \rightarrow \mathbb{w}(s) U(s),
$$

and that $\boldsymbol{G}$ is gauge invariant. The analog of the Berry curvature, the Uhlmann curvature two-form, is defined as

$$
\boldsymbol{F}:=d \boldsymbol{A}+i \boldsymbol{A} \wedge \boldsymbol{A}=\frac{1}{2} \sum_{\mu \nu} F_{\mu \nu} d \lambda_{\mu} \wedge d \lambda_{\nu} .
$$

Its components $F_{\mu \nu}=\partial_{\mu} A_{\nu}-\partial_{\nu} A_{\mu}+i\left[A_{\mu}, A_{\nu}\right]$ can be understood in terms of the Uhlmann holonomy per unit area associated to an infinitesimal loop in the parameter space. Indeed, for an infinitesimal parallelogram $\gamma_{\mu \nu}$, spanned by two independent directions $\hat{e}_{\mu} \delta_{\mu}$ and $\hat{e}_{\nu} \delta_{v}$ in the manifold, it reads

$$
F_{\mu \nu}=\lim _{\delta \rightarrow 0} i \frac{1-V_{\gamma_{\mu, \nu}}}{\delta_{\mu} \delta_{\nu}},
$$

where $\delta \rightarrow 0$ is a shorthand of $\left(\delta_{\mu}, \delta_{v}\right) \rightarrow(0,0)$. While the Abelian Berry curvature $\boldsymbol{F}^{B}$ is a gauge invariant (as one expects from the electromagnetic field analogy), the Uhlmann curvature $\boldsymbol{F}$ is only gauge covariant, i.e. it transforms as

$$
\boldsymbol{F} \rightarrow U^{\dagger}(s) \boldsymbol{F} U(s) \text {, under } \mathbb{w}(s) \rightarrow \mathbb{w}(s) U(s) .
$$

This is a direct consequence of the gauge covariance of any non-Abelian holonomy [26,30],

$$
V_{\gamma}=\mathcal{P} e^{i \oint_{\gamma} A} \rightarrow U_{t}^{\dagger} \mathcal{P} e^{i \oint_{\gamma} A} U_{t} .
$$

The standard approach to the definition of bona-fide observables associated to non-Abelian gauge fields is to resort to the Wilson loop $W_{\gamma}:=\operatorname{Tr} V_{\gamma}$, i.e. the trace of the holonomy operator associated to an arbitrary loop. Thanks to the cyclic property of the trace, the Wilson loop is evidently gauge invariant. It would then be tempting to define a local gauge invariant quantity, analogue of Uhlmann curvature, by considering the Wilson loop per unit area of an infinitesimal loop in the parameter space. This would lead to the trace of curvature, which, however, in the case of the Uhlmann holonomy is always trivial, i.e. $\operatorname{Tr} \boldsymbol{F}=0$.

Alternatively, one may consider another gauge invariant quantity, the Uhlmann geometric phase (119), which in terms of the Uhlmann holonomy reads,

$$
\varphi^{U}[\gamma]:=\arg \operatorname{Tr}\left[\mathbb{w}_{\lambda(0)}^{\dagger} \mathbb{w}_{\lambda(T)}\right]=\arg \operatorname{Tr}\left[\mathbb{w}_{\lambda(0)}^{\dagger} \mathbb{w}_{\lambda(o)} V_{\gamma}\right],
$$


and evaluate the phase per unit area on an infinitesimal closed loop, which reads

$$
\mathcal{U}_{\mu \nu}:=\lim _{\delta \rightarrow 0} \frac{\varphi^{U}\left[\gamma_{\mu \nu}\right]}{\delta_{\mu} \delta_{\nu}}=\operatorname{Tr}\left[\mathrm{w}_{\lambda(0)}^{\dagger}{ }^{\mathbb{w} \lambda(0)} F_{\mu \nu}\right]
$$

This is by definition a gauge invariant. I will call it mean Uhlmann curvature (MUC), on account of the expression

$$
\mathcal{U}_{\mu \nu}=\operatorname{Tr}\left(\rho F_{\mu \nu}\right)=\left\langle F_{\mu \nu}\right\rangle,
$$

that $\mathcal{U}_{\mu \nu}$ takes in the special gauge $\mathrm{w}_{0}=\sqrt{\rho(0)}$.

By taking the external derivative of the expression (142) and by using the property $d^{2}=0$, it can be shown that

$$
\begin{aligned}
0=d^{2} \mathrm{w} & =i_{\mathrm{w}} d \boldsymbol{A}+i d_{\mathrm{w}} \wedge \boldsymbol{A}+d \boldsymbol{G} \mathrm{w}-\boldsymbol{G} \wedge d_{\mathrm{w}} \\
& =i_{\mathrm{w}} d \boldsymbol{A}+i\left(i_{\mathrm{w}} \boldsymbol{A}+\boldsymbol{G}_{\mathrm{w}}\right) \wedge \boldsymbol{A}+d \boldsymbol{G} \mathrm{w}-\boldsymbol{G} \wedge\left(i_{\mathrm{w}} \boldsymbol{A}+\boldsymbol{G}_{\mathrm{w}}\right) \\
& =i_{\mathrm{w}}(d \boldsymbol{A}+i \boldsymbol{A} \wedge \boldsymbol{A})+(d \boldsymbol{G}-\boldsymbol{G} \wedge \boldsymbol{G})_{\mathrm{w}}
\end{aligned}
$$

which leads to [281]

$$
\begin{aligned}
{ }_{\mathrm{w}} \boldsymbol{F} & =i(d \boldsymbol{G}-\boldsymbol{G} \wedge \boldsymbol{G}) \mathbb{w}, \\
\boldsymbol{F}_{\mathbb{W}^{\dagger}} & =-i_{\mathbb{W}}{ }^{\dagger}(d \boldsymbol{G}+\boldsymbol{G} \wedge \boldsymbol{G}) .
\end{aligned}
$$

Multiplying the above expressions by $\mathrm{w}^{\dagger}$ and $\mathrm{w}$, respectively, and taking the trace yields

$$
\boldsymbol{U}=\operatorname{Tr}\left(\mathrm{w} \boldsymbol{F}_{\mathbb{w}^{\dagger}}^{\dagger}\right)=-i \operatorname{Tr}(\rho \boldsymbol{G} \wedge \boldsymbol{G}),
$$

where $\mathcal{U}:=1 / 2 \sum_{\mu \nu} \mathcal{U}_{\mu \nu} d \lambda_{\mu} \wedge d \lambda_{\nu}$ is a real-valued two-form, whose components are

$$
\mathcal{U}_{\mu \nu}=-i \operatorname{Tr}\left(\rho\left[G_{\mu}, G_{\nu}\right]\right) \text {. }
$$

Notice the striking similarity with the expression (133) with which the mean Uhlmann curvature shares the same origin and mathematical structure. Similarly to (135), it is easy to derive an expression for the MUC in the eigenbasis of the density matrix by making use of the expression (134), i.e.

$$
\mathcal{U}_{\mu \nu}=-i \sum_{\substack{p_{j}>0 \\ p_{k}>0}}\left(p_{j}-p_{k}\right) \frac{\left\langle j\left|\partial_{\mu} \rho\right| k\right\rangle\left\langle k\left|\partial_{\nu} \rho\right| j\right\rangle}{\left(p_{j}+p_{k}\right)^{2}} .
$$

From the common mathematical structure of the Bures metric $g_{\mu \nu}$ in (133) and the mean Uhlmann curvature (152), it is quite tempting to define the following positive (semi)-definite Hermitian tensor

$$
Q_{\mu \nu}:=\operatorname{Tr}\left(\rho G_{\mu} G_{v}\right)=g_{\mu \nu}+\frac{i}{2} \mathcal{U}_{\mu \nu} .
$$

This is clearly a mixed state generalisation of the quantum geometric tensor (68) defined in Section 6.1. In the following, we will indistinctly refer to both Eq. (68) and to its mixed state generalisation (see Eq. (154)) as quantum geometric tensor.

\section{Multi-parameter quantum state estimation}

This section aims at providing a different physical perspective to the quantities introduced in the previous sections. It turns out that objects such as the Bures metric and the mean Uhlmann curvature are intimately related to a pivotal quantity in quantum parameter estimation problems, namely the quantum Fisher information.

Estimation theory is the discipline that studies the accuracy by which a given set of physical parameters can be evaluated. When the parameters to be estimated belong to an underlying quantum physical system one falls in the realm of quantum estimation theory, or quantum metrology [293]. Quantum parameter estimation finds applications in a wide variety of fields, from fundamental physics [294-298], to gravitational wave interferometry [299,300], thermometry [301,302], spectroscopy [303,304], imaging [305-307], to name a few. Exploiting remarkable features of quantum systems as probes may give an edge over the accuracy of classical parameter estimation. Exploring this possibility plays a pivotal role in the current development of quantum technology [308-317]. In multi-parameter quantum estimation protocols, several variables are simultaneously evaluated, in a way which may outperform individual estimation strategies with equivalent resources [318,319], thereby motivating the use of such protocols in a variety of diverse contexts [318-321].

The use of peculiar quantum many-body states as probes in quantum metrology can enhance the accuracy in parameter estimation [230,317]. Conversely, one may think of using quantum metrological tools in the study and characterisation of many-body systems. Noteworthy instances of many-body quantum systems are those experiencing quantum phase transitions. Indeed, quantum parameter estimation, with its intimate relation with geometric information, provides a novel and promising approach to investigate equilibrium [31,32,39,42,120,121,158,159,165,322-324] and out-of-equilibrium [269-271,273-275,325-327] quantum critical phenomena [110,113,177,178,192,328]. 
The quantum Fisher information matrix $J(\lambda)$ defines a figure of merit of the estimation precision of parameters labelling a quantum state, known as the quantum Cramér-Rao (CR) bound $[293,311,329]$. Given a set of locally unbiased estimators $\{\hat{\lambda}\}$ of the parameters $\lambda \in \mathcal{M}$, the covariance matrix $\operatorname{Cov}_{\lambda}[\hat{\lambda}]_{\mu \nu}=\left\langle\left(\hat{\lambda}_{\mu}-\lambda_{\mu}\right)\left(\hat{\lambda}_{\nu}-\lambda_{\nu}\right)\right\rangle$ is lower bounded, in a matrix sense, as follows

$$
\operatorname{Cov}_{\lambda}[\hat{\lambda}] \geq J(\lambda)^{-1} \text {. }
$$

It turns out that such a matrix is mathematically equivalent, except for pathological case [289], to the Bures metric tensor $g$, or precisely

$$
J_{\mu \nu}(\lambda)=4 g_{\mu \nu} .
$$

For single parameter estimation, the quantum Cramér-Rao bound (155) can always be saturated by a suitable optimal POVM. However, in a multi-parameter scenario this is not always the case and the above inequality cannot always be attained. This is due to the non-commutativity of measurements associated to independent parameters. It turns out that, within a relatively general setting, known as quantum local asymptotic normality [330-333], the multi-parameter quantum Cramér-Rao bound (155) is attainable iff [190]

$$
\mathcal{U}_{\mu v}=0 \quad \forall \lambda_{\mu}, \lambda_{\nu} .
$$

In this sense, $\mathcal{U}_{\mu \nu}$ marks the incompatibility between $\lambda_{\mu}$ and $\lambda_{\nu}$, and such an incompatibility arises from the inherent quantum nature of the underlying physical system.

From a metrological point of view, $\mathcal{U}_{\mu \nu}$ marks the incompatibility between $\lambda_{\mu}$ and $\lambda_{\nu}$, where such an incompatibility arises from the inherent quantum nature of the underlying physical system.

In particular, one can show that for an $N$-parameter estimation model, the deviation of the attainable multi-parameter bound from the Cramér-Rao bound can be estimated by the quantity $R:=\left\|2 i J^{-1} \mathcal{U}\right\|_{\infty}$, where

$$
0 \leq R \leq 1 .
$$

Indeed, $R$ provides a figure of merit which measures the amount of incompatibility within a parameter estimation model. The lower limit condition, $R=0$, is equivalent to the compatibility condition, i.e. Eq. (151). On the other hand, when the upper bound of Eq. (158) is saturated, i.e. $R=1$, it maximises the discrepancy between the CR bound, that could be attained in an analogous classical multi-parameter estimation problem, and the actual multi-parameter quantum CR bound. In this sense, this bound marks the condition of maximal incompatibility. When this condition is met, the indeterminacy arising from the quantum nature of the estimation problem reaches the order of $\left\|J^{-1}\right\|_{\infty}$, i.e. the same order of magnitude of the Cramér-Rao bound (155). In other words, this implies that the indeterminacy due to the quantum incompatibility arises at an order of magnitude which cannot be neglected.

This is particularly relevant, considering that the scope of optimal schemes is minimising the parameter estimation error. This can only be done by designing strategies which strive for the highest possible rate of growth of $J(n)$ with the number $n$ of available resources. When the condition (155) of maximal incompatibility holds, it implies that the quantum indeterminacy in the parameter estimation problem remains relevant even in the asymptotic limit $n \rightarrow \infty$.

\subsection{Formulation of the problem}

It is often the case that a physical variable of interest is not directly accessible, either for experimental limitations or due to fundamental principles. When this happens one could resort to an indirect approach, inferring the value of the variable after measurements on a given probe. This is essentially a parameter estimation problem whose solution may be found using methods from classical estimation theory [334] or, when quantum systems are involved, from its quantum counterpart [293].

The solution of a parameter estimation problem amounts to find an estimator, i.e. a mapping $\hat{\lambda}=\hat{\lambda}\left(x_{1}, x_{2}, \ldots\right)$, from the set $\chi$ of measurement outcomes into the space of parameters $\lambda \in \mathcal{M}$. Optimal unbiased estimators in classical estimation theory are those saturating the Cramér-Rao (CR) inequality,

$$
\operatorname{Cov}_{\lambda}[\hat{\lambda}] \geq J^{c}(\lambda)^{-1},
$$

which poses a lower bound on the mean square error $\operatorname{Cov}_{\lambda}[\hat{\lambda}]_{\mu \nu}=E_{\lambda}\left[(\hat{\lambda}-\lambda)_{\mu}(\hat{\lambda}-\lambda)_{v}\right]$ in terms of the Fisher information (FI)

$$
J_{\mu \nu}^{c}(\lambda)=\int_{\chi} d \hat{\lambda}(x) p(\hat{\lambda} \mid \lambda) \partial_{\mu} \log p(\hat{\lambda} \mid \lambda) \partial_{\nu} \log p(\hat{\lambda} \mid \lambda) .
$$

The expression (159) should be understood as a matrix inequality. In general, one writes

$$
\operatorname{tr}\left(W \operatorname{Cov}_{\lambda}[\hat{\lambda}]\right) \geq \operatorname{tr}\left(W J^{c}(\lambda)^{-1}\right)
$$

where $W$ is a given positive definite cost matrix, which allows the uncertainty cost of different parameters to be weighted. 
In the classical estimation problem, both in the single parameter case, and in the multi-parameter one, the CR bound can be attained in the asymptotic limit of an infinite number of experiment repetitions using the maximum likelihood estimator [335]. However, an interesting difference between single and multi-parameter metrology arises due to correlations between the variables. Indeed, it may well happen that the off-diagonal elements of the Fisher information matrix are non-vanishing. Hence, there are statistical correlations between the parameter estimators. In a protocol in which all variables but $\lambda_{\mu}$ are precisely known, the single-parameter CR bound implies that the best attainable accuracy in estimating $\lambda_{\mu}$ is given by $\operatorname{Var}(\hat{\lambda}) \geq 1 / J_{\mu \mu}^{c}$. However, in a scenario in which all parameters are simultaneously estimated, one finds that the ultimate precision is lower bounded by $\operatorname{Var}(\hat{\lambda}) \geq\left(J^{c}(\lambda)^{-1}\right)_{\mu \mu}$. A straightforward calculation shows that, for positive-definite matrices, $\left(J^{c}(\lambda)^{-1}\right)_{\mu \mu} \geq 1 / J^{c}(\lambda)_{\mu \mu}$, where the inequality is saturated only for vanishing offdiagonal elements. In the limit of a large number of experiment repetitions the CR bound is attainable. This means that the equivalence between the simultaneous and the individual protocols in the asymptotic limit holds only if the Fisher information is a diagonal matrix, i.e. if the estimators are not correlated [336].

Obviously, any given real positive definite matrix can be transformed via an orthogonal rotation into a diagonal matrix. This clearly implies that there is always a combination of the parameters for which the Fisher information matrix is diagonal. However, this choice should be contrasted with the physical opportunity of performing such a rotation, as the choice of the parameters we are interested in may arise as a result of physical considerations and in this sense determines a preference in a specific basis.

The underlying quantities used in the derivation of classical Fisher information are parameter-dependent probabilitydistributions of the data, whereas the objects involved in the quantum estimation problems are density operators $\rho(\lambda)$ labelled by $\lambda \in \mathcal{M}$. Hence, a further difficulty of quantum estimation protocols is devising the optimal measurement strategy which gathers from the density matrix the greatest amount of information on the labelling parameters. For single parameter estimation, the solution is quite straightforward. If one maximises the classical Fisher information over all possible quantum measurements, the result is the so-called quantum Fisher information (QFI). The key object involved in the calculation of the QFI is the so-called symmetric logarithmic derivative (SLD), $L_{\mu}$, a Hermitian operator which is implicitly defined as the solution of the equation

$$
\partial_{\mu} \rho(\boldsymbol{\lambda})=\frac{1}{2}\left\{\rho(\boldsymbol{\lambda}) L_{\mu}(\boldsymbol{\lambda})+L_{\mu}(\boldsymbol{\lambda}) \rho(\boldsymbol{\lambda})\right\} .
$$

The above equation, apart from a factor $1 / 2$, is identical to the defining Eq. (130) of the parallel transport generator $G_{\mu}$. However, a relatively benign difference between $G_{\mu}$ and $L_{\mu}$ arises from the auxiliary condition (131). This may cause a sizeable discrepancy between their behaviours in some pathological cases, which may occur around points of the state manifold, where $\rho(\boldsymbol{\lambda})$ undergoes a change of rank [289].

The QFI can be calculated using the formula:

$$
J_{\mu \mu}(\boldsymbol{\lambda})=\frac{1}{2} \operatorname{Tr} \rho(\boldsymbol{\lambda})\left\{L_{\mu}(\boldsymbol{\lambda}), L_{\mu}(\boldsymbol{\lambda})\right\} .
$$

One can always choose the projective measurement in the eigenbasis of the SLD which yields FI equal to the QFI. Hence, the QFI determines the ultimate achievable estimation precision of the parameter on density matrices $\rho(\lambda)$ in the asymptotic limit of an infinite number of experiment repetitions. In a multiparameter scenario, a direct generalisation of single parameter CR bound leads to the multiparameter QFI CR bound [293,311,329], that reads

$$
\operatorname{tr}(W \operatorname{Cov}(\hat{\lambda})) \geq \operatorname{tr}\left(W J^{-1}\right),
$$

where

$$
J_{\mu \nu}=\frac{1}{2} \operatorname{Tr} \rho\left\{L_{\mu}, L_{\nu}\right\},
$$

is the quantum Fisher information matrix (QFIM) and $W$ is the cost matrix.

\subsection{Multi-parameter incompatibility: a measure of quantumness}

Unlike the single parameter case, in the multi-parameter scenario the quantum CR bound cannot always be saturated. Intuitively, this is due to the incompatibility of the optimal measurements for different parameters. A sufficient condition for the saturation is indeed $\left[L_{\mu}, L_{v}\right]=0$, which is however not a necessary condition. Within the comprehensive framework of quantum local asymptotic normality (QLAN) [330-333], a necessary and sufficient condition for the saturation of the multi-parameter CRB is given by $\mathcal{U}_{\mu \nu}=0$ for all $\mu$ and $v$ [190].

Here, we show explicitly that the ratio between $\mathcal{U}_{\mu \nu}$ and $J_{\mu \nu}$ provides a figure of merit for the discrepancy between an attainable multi-parameter bound and the single parameter CRB quantified by $J^{-1}$. We will confine ourself to the broad framework of QLAN, in which the attainable multi-parameter bound is given by the so called Holevo Cramér-Rao bound (HCRB) [293,311,329]. For a $N$-parameter model, the HCRB can be expressed as [330]

$$
\operatorname{tr}(W \operatorname{Cov}(\hat{\lambda})) \geq C_{H}(W)
$$


where

$$
C_{H}(W):=\min _{\left\{X_{\mu}\right\}}\left\{\operatorname{tr}(W \operatorname{Re} Z)+\|(W \mathbf{I m} Z)\|_{1}\right\} .
$$

The $N \times N$ Hermitian matrix $Z$ is defined as

$$
Z_{\mu \nu}:=\operatorname{Tr}\left(\rho X_{\mu} X_{v}\right)
$$

where $\left\{X_{\mu}\right\}$ is an array of $N$ Hermitian operators on $\mathcal{H}$ satisfying the unbiasedness conditions $\operatorname{Tr}\left(\rho X_{\mu}\right)=0, \forall \mu$ and $\operatorname{Tr}\left(X_{\mu} \partial_{\nu} \rho\right)=\frac{1}{2} \operatorname{Tr} \rho\left\{X_{\mu}, L_{\nu}\right\}=\delta_{\mu \nu}, \forall \mu, v$, and $\|B\|_{1}$ denotes the sum of all singular values of $B$. If one chooses for $\left\{X_{\mu}\right\}$ the array of operators $\tilde{X}_{\mu}:=\sum_{\nu}\left[J^{-1}\right]_{\mu \nu} L_{\nu}$, it yields

$$
Z \rightarrow \tilde{Z}:=J^{-1} I J^{-1}=J^{-1}-i 2 J^{-1} \mathcal{U} J^{-1},
$$

where $I$ is the matrix of elements $I_{\mu \nu}:=\operatorname{Tr}\left(\rho L_{\mu} L_{v}\right)$, and $\mathcal{U}$ is the skew-symmetric real matrix $\mathcal{U}_{\mu \nu}=\frac{i}{4} \operatorname{Tr}\left(\rho\left[L_{\mu}, L_{v}\right]\right)$. If one indicates by $\mathcal{D}(W):=C_{H}(W)-\operatorname{tr}^{-1}$ the discrepancy between the attainable multi-parameter HCRB and the CRB, one can write the following bounds

$$
0 \leq \mathcal{D}(W) \leq 2\left\|W J^{-1} \mathcal{U} J^{-1}\right\|_{1} \leq \operatorname{tr}\left(W J^{-1}\right) R,
$$

where $R=\left\|2 \mathrm{iUJ} J^{-1}\right\|_{\infty}$, and the first inequality is saturated iff $\mathcal{U}=0$ [190].

One can show that [111]

$$
0 \leq R \leq 1
$$

When the upper bound (170) is saturated, i.e. condition $R=1$ is met, it implies that

$$
\mathcal{D}(W) \simeq \operatorname{tr}\left(W J^{-1}\right),
$$

which means that the discrepancy $\mathcal{D}(W)$ reaches the same order of magnitude of the CR bound itself. This limit marks the condition of maximal incompatibility for the two-parameter estimation problem, arising from the quantum nature of the underlying system. In the opposite limit $R=0$, the parameter model is compatible, and the discrepancy between the quantum CR bound, and its classical counterpart vanishes. Therefore, $R$ provides a figure of merit which quantifies the quantum contribution to the indeterminacy of multi-parameter estimations.

Proof of Eq. (170). The lower bound comes straightforwardly from Eq. (169). For the upper bound, notice that $Z=\left\{Z_{\mu \nu}\right\}$ in Eq. (167) is a positive semi-definite matrix, since $\forall \vec{a}=\left\{a_{\mu}\right\}_{\mu=1}^{N} \in \mathbb{C}^{N}, \vec{a}^{\dagger} \cdot Z \cdot \vec{a}=\operatorname{Tr}\left(\rho A^{\dagger} A\right) \geq 0$, with $A:=\sum_{\mu} a_{\mu} X_{\mu}$. Then, from Eq. (168),

$$
J^{1 / 2} \tilde{Z} J^{1 / 2}:=\mathbb{1}-i 2 J^{-1 / 2} \mathcal{U} J^{-1 / 2} \geq 0 .
$$

Since $i 2 J^{-1 / 2} \mathcal{U} J^{-1 / 2}$ is a skew-symmetric Hermitian matrix, its eigenvalues are either zero or real numbers that occur in \pm pairs. Then, from Eq. (172) we deduce that these eigenvalues lie within the interval $\{-1,1\}$. Moreover, $J^{-1} \mathcal{U}$ is a diagonalisable matrix with the same eigenvalues of $J^{-1 / 2} \mathcal{U} J^{-1 / 2}$. Indeed, if $J^{-1 / 2} \mathcal{U} J^{-1 / 2}=U^{\dagger} D U$, with $D$ diagonal, then $J^{-1} \mathcal{U}=S^{-1} D S$, where $S=U J^{1 / 2}$. Hence, $R=\left\|i 2 J^{-1} \mathcal{U}\right\|_{\infty}=\left\|i 2 J^{-1 / 2} \mathcal{U} J^{-1 / 2}\right\|_{\infty} \leq 1$

For the special case of a two-parameter model, in the eigenbasis of $J$, with eigenvalues $j_{1}$ and $j_{2}$, it holds

$$
2 i J^{-1} \mathcal{U}=\left(\begin{array}{cc}
j_{1}^{-1} & 0 \\
0 & j_{2}^{-1}
\end{array}\right)\left(\begin{array}{cc}
0 & \mathcal{U}_{12} \\
-\mathcal{U}_{12} & 0
\end{array}\right)=\left(\begin{array}{cc}
0 & 2 i \frac{\mathcal{U}_{12}}{j_{1}} \\
-2 i \frac{\mathcal{U}_{12}}{j_{2}} & 0
\end{array}\right) .
$$

It follows that

$$
R=\left\|2 i J^{-1} \mathcal{U}\right\|_{\infty}=\sqrt{\frac{\operatorname{Det} 2 \mathcal{U}}{\operatorname{Det} J}} .
$$

Hence, $\sqrt{\text { Det } 2 \mathcal{U} / \text { Det } J}$ provides a figure of merit which measures the amount of incompatibility between two independent parameters in a quantum two-parameter model.

For self-adjoint operators $B_{1}, \ldots, B_{N}$, the Schrodinger-Robertson's uncertainty principle is the inequality [337]

$$
\operatorname{Det}\left[\frac{1}{2} \operatorname{Tr} \rho\left\{B_{\mu}, B_{\nu}\right\}\right]_{\mu, \nu=1}^{N} \geq \operatorname{Det}\left[-\frac{i}{2} \operatorname{Tr} \rho\left[B_{\mu}, B_{\nu}\right]\right]_{\mu, \nu=1}^{N},
$$

which, applied to the SLD $L_{\mu}$ 's, yields

$$
\text { Det } J \geq \operatorname{Det} 2 \mathcal{U} \text {. }
$$

For $N=2$, the inequality (176) is equivalent to the upper-bound of Eq. (170), and if saturated, it implies the condition of maximal incompatibility for the two-parameter estimation problem. 
Another interesting inequality relates the eigenvalues of $J$ (and hence of $g$ ) with those of $\mathcal{U}$. The QGT ${ }^{3}$

$$
Q_{\mu \nu}:=\operatorname{Tr} \rho G_{\mu} G_{\nu}=\frac{1}{4} \operatorname{Tr} \rho L_{\mu} L_{\nu}=\frac{1}{4} J_{\mu \nu}+\frac{i}{2} \mathcal{U}_{\mu \nu}
$$

is a positive (semi)-definite Hermitian matrix. Hence, by definition $J \geq-2 i \mathcal{U}$, in a matrix sense. It follows that [338]

$$
\|J\|_{\infty} \geq 2\|i \mathcal{U}\|_{\infty} .
$$

\section{Thermal states and classical phase transitions}

In this section, we would like to give a glance at the extension of the geometric information approach to finite temperature. This approach is indeed directly linked to the approach developed in classical phase transitions [163,339]. The natural generalisation of the geometric information approach to finite temperature is done by replacing the FubiniStudy metric with its mixed state generalisation, the Bures metric. In temperature driven phase transitions, the density matrices $\rho_{\beta_{1}}$ and $\rho_{\beta_{2}}$ at different temperatures $\beta_{i}=\left(\kappa_{B} T_{i}\right)^{-1}$ generally commute ${ }^{4}$, and the Uhlmann fidelity $\mathcal{F}\left(\rho_{\beta_{1}}, \rho_{\beta_{2}}\right):=$ $\operatorname{Tr}\left[\rho_{\beta_{2}}^{1 / 2} \rho_{\beta_{1}} \rho_{\beta_{2}}^{1 / 2}\right]^{1 / 2}$ reduces to the Bhattacharyya coefficient $\mathcal{F}\left(\rho_{0}, \rho_{1}\right)=\sum_{k} \sqrt{p_{k}^{1} p_{k}^{2}}$, where the $p_{k}^{i}$ are the eigenvalues of $\rho_{\beta_{i}}$. Correspondingly, when two states differing just by two infinitesimally closed temperatures $\beta_{1}=\beta$ and $\beta_{2}=\beta+d \beta$ are considered, the Bures metric collapses into the Fisher-Rao metric,

$$
d l_{B}=\frac{1}{4} \sum_{p_{k}>0} \frac{\left(\partial_{\beta} p_{k}\right)^{2}}{p_{k}} d \beta^{2}=\frac{1}{4}\left[\left\langle H^{2}\right\rangle-\langle H\rangle^{2}\right] d \beta^{2} .
$$

In particular, when $\rho_{\beta}=Z^{-1}(\beta) \exp (-\beta H)=Z^{-1}(\beta) \sum_{k} e^{-\beta E_{k}}|k\rangle\langle k|$, with $Z(\beta):=\operatorname{Tr} \exp (-\beta H)$, where $E_{k}$ and $|k\rangle$ are the eigenvalues and eigenvectors of the Hamiltonian, one easily sees that

$$
\partial_{\beta} p_{k}=\partial_{\beta}\left(\frac{e^{-\beta E_{k}}}{Z}\right)=-p_{k}\left[E_{k}-\left(\sum_{j} E_{j} p_{j}\right)\right]=p_{k}\left[\langle H\rangle_{\beta}-E_{k}\right],
$$

therefore (179) can be written as [159,163,193,339]

$$
d l_{B}=\frac{1}{4} \sum_{k} p_{k}\left[\langle H\rangle_{\beta}-E_{k}\right]^{2} d \beta^{2}=\frac{1}{4}\left[\left\langle H^{2}\right\rangle_{\beta}-\langle H\rangle_{\beta}^{2}\right] d \beta^{2}=\frac{d \beta^{2}}{4} T^{2} c_{V}(\beta) .
$$

This is quite a remarkable relation which provides a connection between a geometric information measure of distinguishability and a macroscopic thermodynamic quantity $c_{V}$.

More importantly, it also provides a direct connection with classical critical phenomena. Thermal phase transitions are signalled by non-analytical behaviour of the specific heat, which may be picked up as singularity of the Bures/Fisher-Rao metric. This simple fact shows that information geometry at large provides a comprehensive framework under which both quantum and classical critical phenomena can be readily investigated.

\section{Susceptibility and mean Uhlmann curvature}

The MUC is a geometrical quantity, whose definition relies on a rather formal definition of holonomies of density matrices. In spite of its abstract formalism, the MUC has interesting connections to a physically relevant object which is directly observable in experiments, the susceptibility. In a thermal equilibrium setting, one can rigorously relate the MUC to the dissipative part of the dynamical susceptibility [118,119]. Indeed, by using linear response theory, one can consider the most general scenario of a system with a Hamiltonian $\mathcal{H}_{0}$, perturbed as follows

$$
\mathcal{H}=\mathcal{H}_{0}+\sum_{\mu} \hat{O}_{\mu} \lambda_{\mu}
$$

where $\left\{\hat{O}_{\mu}\right\}$ is a set of observables of the system, and $\left\{\lambda_{\mu}\right\}$ is the corresponding set of perturbation parameters. We are considering the system in thermal equilibrium, i.e. $\rho=\frac{e^{-\beta \mathcal{H}}}{\mathcal{Z}}$, where $\mathcal{Z}=\operatorname{Tr}\left[e^{-\beta \mathcal{H}}\right]$ is the partition function. The dissipative part of the dynamical susceptibility, with respect to $\hat{O}_{\mu}$, is defined as:

$$
\chi_{\mu \nu}^{\prime \prime}(t)=\frac{1}{2 \hbar}\left\langle\left[\hat{O}_{\mu}(t), \hat{O}_{\nu}\right]\right\rangle_{0},
$$

\footnotetext{
3 We are deliberately assuming $L_{\mu}=2 G_{\mu}$, thus neglecting possible discrepancy arising due to variations in the rank of $\rho$ [289].

4 Here one considers Hamiltonians which do not explicitly depend on temperature. For example, effective mean field Hamiltonians with temperature dependent parameters may result in non-commuting density matrices $\left[\rho_{\beta_{1}}, \rho_{\beta_{2}}\right] \neq 0$. The BCS Hamiltonians are one such case [185].
} 
One can show that the Fourier transform of the dissipative part of the dynamical susceptibility has the following expression in the Lehmann representation

$$
\chi_{\mu \nu}^{\prime \prime}(\omega, \beta)=\frac{\pi}{\hbar} \sum_{i j}\left(\hat{O}_{\mu}\right)_{i j}\left(\hat{O}_{v}\right)_{j i}\left(p_{i}-p_{j}\right) \delta\left(\omega+\frac{E_{i}-E_{j}}{\hbar}\right),
$$

where $p_{i}$ 's are the eigenvalues of the density matrix in the Boltzmann-Gibbs ensemble, i.e. $p_{i}=e^{-\beta E_{i}} / Z$, and $E_{i}$ 's are the corresponding Hamiltonian eigenvalues. For thermal states, one can exploit the identity

$$
\frac{p_{i}-p_{j}}{p_{i}+p_{j}}=\int_{-\infty}^{+\infty} d \omega \tanh \left(\frac{\hbar \omega \beta}{2}\right) \delta\left(\omega+\frac{E_{i}-E_{j}}{\hbar}\right),
$$

which leads to the following relation between the $\chi_{\mu \nu}^{\prime \prime}(\omega, \beta)$ and the MUC,

$$
\mathcal{U}_{\mu \nu}=\frac{i}{\hbar \pi} \int_{-\infty}^{+\infty} \frac{d \omega}{\omega^{2}} \tanh ^{2}\left(\frac{\hbar \omega \beta}{2}\right) \chi_{\mu \nu}^{\prime \prime}(\omega, \beta),
$$

where the set of perturbations $\left\{\lambda_{\mu}\right\}$ in (182) plays the role of the parameters in the derivation of $\mathcal{U}_{\mu \nu}$. By means of the fluctuation-dissipation theorem [340], one can further derive an expression for Eq. (186) in terms of the dynamical structure factor, $S_{\mu \nu}(\omega, \beta)=\int_{-\infty}^{+\infty} d t e^{i \omega t} S_{\mu \nu}(t)$ (i.e. the Fourier transform of the correlation matrix $S_{\mu \nu}(t)=\left\langle\hat{O}_{\mu}(t) \hat{O}_{v}(0)\right\rangle$ ), which reads

$$
\mathcal{U}_{\mu \nu}=\frac{i}{2 \pi \hbar} \int_{-\infty}^{+\infty} \frac{d \omega}{\omega^{2}} \tanh ^{2}\left(\frac{\hbar \omega \beta}{2}\right)\left(S_{\mu \nu}(\omega, \beta)-S_{\nu \mu}(-\omega, \beta)\right) .
$$

Eqs. (186) and (187) provide a means to explore experimentally the geometrical properties of physical systems via the dissipative part of the dynamical susceptibility, and the imaginary part of the (off-diagonal)-dynamical structure factor. The dynamical susceptibility is a well studied quantity which has been observed experimentally in a wide variety of materials [341-346]. Using such an experimentally accessible quantity provides a robust method to explore the geometric features of criticality in strongly correlated materials.

\section{Dissipative non-equilibrium phase transitions}

In contrast to equilibrium critical phenomena, less is known in case of non-equilibrium phase transitions. Indeed, a generalised treatment is not known, lacking an analog to the equilibrium free energy. Thus the rich and often surprising variety of non-equilibrium phase transitions observed in physical [347-352], chemical, biological [353-358], as well as socioeconomic systems [359-361], has to be studied for each system individually.

The paradigm of universality, legitimised by its astounding success in equilibrium phase transitions, does not find an equivalently comprehensive framework within non-equilibrium phenomena. This results in a large variety of universality classes without general tools for their characterisation [362,363]. For instance, algebraically decaying correlation functions are not peculiar of critical phenomena [364]. Also the spectral gap of the Liouvillian, an open system generalisation of the Hamiltonian gap, may vanish in the thermodynamic limit in the whole phase diagram, with critical points resulting only in a faster convergence [123,365].

The specific domain of quantum many-body physics in recent years has witnessed a growing interest in nonequilibrium phenomena. The reason can be credited to at least two main causes. On the one hand, the unprecedented level of accuracy reached in nowadays experimental techniques provides many mature platforms for the investigation and manipulation of many-body interacting systems. Indeed, several set of available tools have enabled the development of Liouvillian engineering, which in addition to coherent Hamiltonian dynamics also includes controlled dissipation in many-body quantum systems. Examples of suitable experimental platforms for the implementation and simulation of such an open many-body framework range from ultra-cold atoms in optical lattices [124,366], to ion traps [367,368], to cavity microarrays [143,369-374] and Rydberg atoms [375,376]. On the other hand, the study of non-equilibrium quantum phenomena can arguably be related to important open challenges in many-body physics, ranging from high temperature super-conductivity to quantum computation in condensed matter setups.

There are two major framework within which non-equilibrium quantum many-body physics are generally investigated. One of the most popular approach [377] considers a large, ideally infinite, system which is initially kept in an equilibrium state. A perturbation may then be applied to the system either via a sudden quench of the system Hamiltonian, or by a periodic application of an external field, or still by the coupling to a suitably structured reservoir. The resultant system time evolution is then observed, and the outset - or the lack thereof - of stationary or metastable long-time behaviour provides several quantitative and qualitative information on the non-equilibrium properties of the many-body system.

Another, more direct approach, which we will consider here, consists instead in coupling a finite or infinite system to several external reservoirs which may be described effectively in terms of a master equation [154,155]. Under 
suitable conditions, the open system dynamics reaches a possibly unique non-equilibrium steady state. The dynamical and static properties of the NESSes to which the system eventually relaxes are the central object of this second course of investigations.

There are several techniques involving many levels of assumptions and approximations in deriving the effective system's dynamics of open systems interacting with a reservoir [154,155]. The standard approach, mostly used in quantum optical settings, results in a local-in-time Markovian linear differential equations for the system's density matrix, the socalled quantum Liouville equation. The most general Markovian form of such an equation is sometimes referred to as the Redfield equation. A more mathematically appealing form, which manifestly preserves the complete positivity of the density matrix, and can be derived from the Redfield model with the additional secular approximation, is the so called Lindblad equation [378].

In this setting, non-equilibrium criticalities are identified as dramatic structural changes of the Liouvillian steady state due to small modification of tuneable external parameter of the system. The analogy with equilibrium phase transitions is straightforward. For zero temperature, QPTs are understood through the properties of the (unique) ground state of the Hamiltonian $\mathcal{H}(\lambda), \lambda \in \mathcal{M}$ governing the dynamics of the system,

$$
\frac{d \rho}{d t}=-i[\mathcal{H}(\lambda), \rho] .
$$

Phase diagram and criticality are determined by the low-lying spectrum of excitations of the system's Hamiltonian. It is the singular properties of the ground state, as a function of the Hamiltonian parameters $\lambda \in \mathcal{M}$, which are associated to the macroscopic observable effects typical of criticality. These manifest themselves through divergence of correlation length and generally occur if the gap of the Hamiltonian closes.

Similarly, in a dynamics governed by a Liouvillian master equation

$$
\frac{d \rho}{d t}=\mathcal{L}(\boldsymbol{\lambda}) \rho,
$$

which generally depends on a set of external parameters $\lambda \in \mathcal{M}$, the family of (possibly unique) NESSes $\rho_{s}(\lambda)$ are themselves labelled by the same parameters. Observable macroscopical behaviour in the physical properties of a many-body quantum system are associated to non-analytical dependences of $\rho(\lambda)$ in the manifold $\mathcal{M}$.

From a mathematical point of view, the Liouvillian $\mathcal{L}(\lambda)$ is a linear non-Hermitian super-operator acting on the space of density matrices, and a NESS is defined as its "eigen-density-matrix" with zero eigenvalue,

$$
\mathcal{L}(\lambda) \rho_{s}(\lambda)=0 .
$$

The spectral resolution of the Liouvillian operator $\mathcal{L}_{\lambda}$ provides information on the uniqueness of the stable state and on the asymptotic decay rates which governs the system's relaxation towards the NESS(es). The smallest of such rates, denoted by $\Delta_{\mathcal{L}}$, is the so called Liouvillian spectral gap and determines the dominant time scale $\tau_{c} \sim \Delta_{\mathcal{L}}^{-1}$ of the dissipative dynamics. This quantity is the closest open system analogue to the Hamiltonian gap. Pretty much in the spirit of QPTs, NESS criticalities are accompanied by the vanishing of $\Delta_{\mathcal{L}}$, a phenomenon known as critical slow down. Although generally accepted as an indicator of dissipative phase transitions at large [124,126,379-382], a vanishing dissipative gap is not a distinguishing feature of NESS criticality, and it may be observed across phases characterised by short range correlations $[110,123,178,364,365]$.

Now we will consider systems whose interaction with an environment leads to a time evolution governed by a Lindblad master equation [155],

$$
\frac{d \rho}{d t}=\mathcal{L} \rho=-i[\mathcal{H}, \rho]+\sum_{\alpha}\left(2 \Lambda_{\alpha} \rho \Lambda_{\alpha}^{\dagger}-\left\{\Lambda_{\alpha}^{\dagger} \Lambda_{\alpha}, \rho\right\}\right)
$$

where $\rho$ is the density matrix of the system, $\mathcal{H}$ is its Hamiltonian, and the Lindblad operators (or jump operators) $\Lambda_{\alpha}$ determine the interaction between the system and the bath. The dissipative dynamics is completely determined by the jump operators $\Lambda_{\alpha}$, whose physical origin is prone to different interpretations: From a microscopic point of view they can be regarded as the effective action of a full Hamiltonian dynamics of system and bath, where the degrees of freedom of the reservoir have been traced out. Here, three major approximations are needed: System and environment are initially in an uncorrelated state, system and bath interacts weakly (Born approximation), and the equilibration time of environment is short compared to other time scales (Markov approximation). A second more versatile interpretation originates from the concept of digital simulators [373,375,383-387], where a set of arbitrary (local) Lindblad operators $\Lambda_{\alpha}$ can be explicitly implemented in terms of local, tailored interactions [375]. From a mathematical point of view, Eq. (191) is the most general form of time evolution described by a quantum dynamical semigroup, i.e., a family of completely positive trace-preserving maps $\mathcal{E}_{t}$, which is strongly continuous and satisfies $\mathcal{E}_{t_{1}} \mathcal{E}_{t_{2}}=\mathcal{E}_{t_{1}+t_{2}}[154,378]$.

Despite the above mentioned analogies, however, non-equilibrium QPTs are of a nature different from the standard QPTs at zero temperature, and their investigation requires a substantial change of approach, both conceptually and methodologically. At a conceptual level, stationary states are the result of coherent dynamics dominated by incoherent dissipative processes. The response to a small perturbation is primarily described by relaxation mechanisms rather than the result of adiabatic modifications of the (ground) state. From a technical point of view, the non-Hermitian nature of 
the Liouvillian superoperator $\mathcal{L}$, as opposed to pure eigenvectors of a Hermitian Hamiltonian operator $H$, calls for the development of alternative strategies, as the usual spectral theorem and perturbation theory simply do not apply. This is quite a daunting task to tackle in its full generality.

However, by confining oneself to the physically relevant case of quadratic Liouvillian models of Fermions and spin lattices one is able to state results with a significant level of generality [191,380,381]. This parallels the central role that quasi-free models play in the standard theory of quantum phase transitions. Specific models belonging to this class indeed display rich non-equilibrium features, non-trivial topological properties and NESS-QPTs [123,124,381,382].

\section{GaussIan fermionic states}

Let us make a brief detour to introduce the formalism of Gaussian Fermionic states (GFS). To this end we consider systems of $n$ Fermionic particles described by creation and annihilation operators $c_{j}^{\dagger}$ and $c_{j}$. These operators obey the canonical anti-commutation relations,

$$
\left\{c_{j}, c_{k}\right\}=0 \quad\left\{c_{j}, c_{k}^{\dagger}\right\}=\delta_{j k} .
$$

A convenient formulation for quadratic models is in terms of the Hermitian Majorana operators, which are defined as

$$
w_{2 j-1}:=c_{j}+c_{j}^{\dagger}, \quad w_{2 j}:=i\left(c_{j}-c_{j}^{\dagger}\right),
$$

which, as generators of a Clifford algebra, satisfy the following anti-commutation relations

$$
\left\{w_{j}, w_{k}\right\}=2 \delta_{j k} .
$$

We will consider an Hamiltonian quadratic in the Fermionic operators

$$
\mathcal{H}:=\sum_{j k=1}^{2 n} H_{j k} w_{j} w_{k}=\boldsymbol{w}^{T} H \boldsymbol{w}, \quad H=H^{\dagger}=-H^{T},
$$

where $\boldsymbol{w}:=\left(w_{1} \ldots w_{2 n}\right)^{T}$ is an array of Majorana operators and $H$ is a $2 n \times 2 n$ Hermitian antisymmetric matrix. Quadratic Hamiltonian models describe quasi-free Fermions and are known to be exactly solvable. Their ground states and thermal states are Gaussian Fermionic states. Indeed, Gaussian Fermionic states are defined as states that can be expressed as

$$
\rho=\frac{e^{-\frac{i}{4} \boldsymbol{w}^{T} \Omega \boldsymbol{w}}}{Z}, \quad Z:=\operatorname{Tr}\left[e^{-\frac{i}{4} \boldsymbol{w}^{T} \Omega \boldsymbol{w}}\right]
$$

where $\Omega$ is a $2 n \times 2 n$ real antisymmetric matrix (see Appendix A). The thermal state of the quadratic model is obtained by the simple identification $\Omega=-4 i \beta H$. Obviously, the converse is always true, a Gaussian state is the thermal state of a suitable quadratic Hamiltonian, which is sometime referred to as parent Hamiltonian [382]. Gaussian states are completely specified by the two-point correlation function

$$
\Gamma_{j k}:=1 / 2 \operatorname{Tr}\left(\rho\left[w_{j}, w_{k}\right]\right), \quad \Gamma=\Gamma^{\dagger}=-\Gamma^{T},
$$

where the matrix $\Gamma:=\left\{\Gamma_{j k}\right\}_{1}^{2 n}$ is a $2 n \times 2 n$ imaginary antisymmetric matrix. All higher-order correlation functions of a Gaussian state can be obtained from $\Gamma$ by Wick's theorem [388].

One can show that $\Gamma$ and $\Omega$ can be simultaneously cast in a canonical form by an orthogonal matrix $Q, Q^{T}=Q^{-1}$,

$$
\Gamma=Q \bigoplus_{k=1}^{n}\left(\begin{array}{cc}
0 & i \gamma_{k} \\
-i \gamma_{k} & 0
\end{array}\right) Q^{T}, \quad \Omega=Q \bigoplus_{k=1}^{n}\left(\begin{array}{cc}
0 & \Omega_{k} \\
-\Omega_{k} & 0
\end{array}\right) Q^{T}
$$

where $\pm \gamma_{k}$ are the eigenvalues of $\Gamma$ and $\pm i \Omega_{k}$ are the eigenvalues of $\Omega$. Indeed, the two matrices are related as,

$$
\Gamma=\tanh \left(i \frac{\Omega}{2}\right) \text {. }
$$

and their eigenvalues can be expressed as $\gamma_{k}=\tanh \left(\Omega_{k} / 2\right)$, which implies that $\left|\gamma_{k}\right| \leq 1$. Moreover let

$$
\boldsymbol{z}=\left(z_{1}, \ldots, z_{2 n}\right)^{T}:=Q \boldsymbol{w}
$$

be the Majorana Fermions in the eigenmode representation. With respect to these Fermions the Gaussian state can be expressed as,

$$
\rho=\prod_{k} \frac{1-i \gamma_{k} z_{2 k-1} z_{2 k}}{2}
$$

Hence, a Gaussian Fermionic state can be factorised into a tensor product $\rho=\bigotimes_{k} \rho_{k}$ of density matrices of the eigenmodes $\rho_{k}:=\frac{1-i \gamma_{k} z_{2 k-1} z_{2 k}}{2}$. Notice that for $\gamma_{k}= \pm 1$, one has $\Omega_{k}= \pm \infty$, making the definition (196) of Gaussian state not well defined, unlike Eq. (201), showing that the latter offers a regular parameterisation even in those extremal points. Notice 
that $\left|\gamma_{k}\right|=1$ corresponds to a Fermionic mode $\tilde{c}_{k}=1 / 2\left(z_{2 k-1}+z_{2 k}\right)$ being in a pure state, as it is clear from the following explicit expression for the purity of the states $\rho_{k}$,

$$
\operatorname{Tr}\left[\rho_{k}^{2}\right]=\frac{1+\gamma_{k}^{2}}{2}
$$

This implies the following basis-independent expression of the purity

$$
\operatorname{Tr}\left[\rho^{2}\right]=\prod_{k} \frac{1+\gamma_{k}^{2}}{2}=\sqrt{\operatorname{det}\left(\frac{\mathbb{1}+\Gamma^{2}}{2}\right)} .
$$

\section{Dissipative Markovian quadratic models}

We will discuss dissipative Fermionic models, described by a Lindblad master equation

$$
\frac{d \rho}{d t}=\mathcal{L} \rho=-i[\mathcal{H}, \rho]+\sum_{\alpha}\left(2 \Lambda_{\alpha} \rho \Lambda_{\alpha}^{\dagger}-\left\{\Lambda_{\alpha}^{\dagger} \Lambda_{\alpha}, \rho\right\}\right)
$$

whose global dynamics is quadratic in Fermion operators. This means that the Hamiltonian considered will be of the type (195), and the set of jump operators $\Lambda_{\alpha}$ will be linear in the Fermion operators, i.e.

$$
\Lambda_{\alpha}=\boldsymbol{l}_{\alpha}^{T} \boldsymbol{w}, \text { with } \boldsymbol{l}_{\alpha}:=\left(l_{1}^{\alpha}, \ldots, l_{2 n}^{\alpha}\right)^{T},
$$

where $\boldsymbol{l}_{\alpha}$ denotes a set of 2 n-dimensional complex vectors. We assume that $H$ and $\boldsymbol{l}_{\alpha}$ 's depend on a set of parameters $\lambda \in \mathcal{M}$ which defines the underlying dissipative model. Due to the quadratic dependence on the Fermionic operators, the Liouvillian can be diagonalised exactly and its stable state is Gaussian. This has been proven in full generality in Ref. [177,389-391] using a formalism called "third quantisation". This essentially consists in the development of an operator algebra acting on the space of density matrices which mimics the algebraic properties of the Fermionic operators acting on the ordinary Fock space. In the following, we will review the way in which the stable state $\rho_{s}$ is obtained within this formalism, which will provide the natural parameterisation necessary for the subsequent developments. The Liouvillian [390] can be written as a quadratic form in terms of the following set of $2 n$ creation and annihilation super-operators

$$
\hat{a}_{j}^{\dagger} \cdot:=-\frac{i}{2} W\left\{w_{j}, \cdot\right\}, \quad \hat{a}_{j} \cdot:=-\frac{i}{2} W\left[w_{j}, \cdot\right],
$$

where $[.,$.$] and \{.,$.$\} are the usual commutator and anti-commutator, respectively and$

$$
W:=i^{n} \prod_{j=1}^{2 n} w_{j}
$$

is an Hermitian operator satisfying the following properties

$$
W=W^{\dagger}, \quad W^{2}=\mathbb{1}, \quad\left\{W, w_{k}\right\}=0 \quad \forall k .
$$

From the above properties one can prove that the super-operators defined in Eq. (206) satisfy the canonical anticommutation relations,

$$
\left\{\hat{a}_{j}, \hat{a}_{k}\right\}=0, \quad\left\{\hat{a}_{j}^{\dagger}, \hat{a}_{k}\right\}=\delta_{j k},
$$

and thus they reproduce the algebra of ordinary Fermionic operators. Notice, however, that the space on which $\hat{a}_{k}$ and $\hat{a}_{k}^{\dagger}$ act is the Hilbert-Schmidt space of linear operator $\mathcal{B}(\mathcal{H})$, to which the set of density matrices $\rho$ belongs.

Let us denote by $\mathcal{R}$ the $4^{n}$-dimensional subspace of $\mathcal{B}(\mathcal{H})$ spanned by $\prod_{j} w_{j}^{s_{j}},\left(s_{j} \in\{0,1\}\right)$. One can regard this subspace as a linear Hilbert space whose elements, denoted by $\mid \boldsymbol{s})$ are normalised with respect to the Hilbert-Schmidt inner product, i.e. $(\boldsymbol{s} \mid \boldsymbol{s}) \equiv \operatorname{Tr}\left[s^{\dagger} s\right]=1$ for $\left.\mid \boldsymbol{s}\right) \in \mathcal{R}$. Notice that the vacuum of the Fermionic super-operators, i.e. the state $\left.\mid 0\right)$ such that $\left.\hat{a}_{k} \mid 0\right)=0$, corresponds to the completely mixed state $\left.\mid 0\right) \propto \mathbb{1}$. Moreover, one can verify that the superoperator $a_{k}^{\dagger}$ is the Hermitian conjugate of $a_{k}$ in $\mathcal{R}$.

Using the above formalism, a lengthy but straightforward calculation shows that the Lindbladian equation Eq. (204) can be explicitly expressed in the following bilinear form in terms of $\hat{a}_{k}$ and $\hat{a}_{k}^{\dagger}$,

$$
\mathcal{L}=-\sum_{j k}\left(X_{j k} \hat{a}_{j}^{\dagger} \hat{a}_{k}+Y_{i j} \hat{a}_{j}^{\dagger} \hat{a}_{k}^{\dagger} / 2\right)
$$

where

$$
\begin{aligned}
& X:=4[i H+\operatorname{Re}(M)], \quad X=X^{*} \\
& Y:=-i 8 \operatorname{Im}(M), \quad Y=Y^{\dagger}=-Y^{T}
\end{aligned}
$$


and

$$
M_{j k}:=\left(\sum_{\alpha} \boldsymbol{l}_{\alpha} \otimes \boldsymbol{l}_{\alpha}^{\dagger}\right)_{j k}=\sum_{\alpha} l_{j}^{\alpha}\left(l_{k}^{\alpha}\right)^{*}, \quad M=M^{\dagger} \geq 0,
$$

is a positive semidefinite matrix called bath matrix.

Under certain conditions derived in [390], the dissipative dynamics admits a unique non-equilibrium steady state solution $\rho_{s}$ such that $\mathcal{L} \rho_{s}=0$, and such a state is Gaussian. The two point correlation function of the NESS is obtained from the solution of the following (continuous) Lyapunov equation:

$$
X \Gamma+\Gamma X^{T}=Y .
$$

As shown in the next section the correlation matrix $\Gamma$ plays also a central role in the diagonalisation of the Liouvillian.

Notice that the real matrix $X$ does not have to be diagonalisable. However, for convenience one can safely assume that this is the case. Indeed, in the explicit models that we will consider in the next sections, this assumption is always satisfied. Nevertheless, the generality of the consideration that will follow will not be affected if this condition is lifted as it will be argued in the Appendix $B$.

Let $\boldsymbol{a}:=\left(\hat{a}_{1}, \ldots, \hat{a}_{2 n}\right)^{T}$ be the array of Fermionic annihilation super-operators, and let $U$ be the invertible matrix that diagonalises $X$, i.e.

$$
X=U D_{X} U^{-1} \quad D_{X}:=\operatorname{diag}\left(\left\{x_{k}\right\}_{k=1}^{2 n}\right),
$$

where $x_{k} \in \mathbb{C}$ are the eigenvalues of $X$. One can show that the following non-unitary Bogoliubov transformation [392],

$$
\left\{\begin{array}{l}
\boldsymbol{b}=U^{-1}\left(\boldsymbol{a}-\Gamma \boldsymbol{a}^{\dagger}\right), \\
\boldsymbol{b}^{\times}=U^{T} \boldsymbol{a}^{\dagger}
\end{array}\right.
$$

where $\boldsymbol{b}:=\left(\hat{b}_{1}, \ldots, \hat{b}_{2 n}\right)^{T}$, brings $\mathcal{L}$ to the diagonal form

$$
\mathcal{L}=-\sum_{k} x_{k} \hat{b}_{k}^{\times} \hat{b}_{k}
$$

Notice that, due to the non-unitarity of the Bogoliubov transformation, the operators $\hat{b}_{j}$ and $\hat{b}_{j}^{\times}$satisfy the canonical anti-commutation relations, however $\hat{b}_{j}^{\times} \neq \hat{b}_{j}^{\dagger}$. Pretty much in the spirit of the standard Bogoliubov transformation, the unnormalised steady state $\rho_{s}$ is the vacuum of the annihilation super-operators $\boldsymbol{b}$, i.e. $\left.\hat{b}_{j} \mid \rho_{s}\right)=0, \forall j=1, \ldots, 2 n$. From the operator form of the Bogoliubov transformation [392], one finds

$$
\rho_{s}=e^{-\frac{1}{2} \boldsymbol{a}^{\dagger} \cdot \Gamma \boldsymbol{a}^{\dagger}}(\mathbb{1})
$$

where, as noted earlier, the identity operator is the vacuum of $\boldsymbol{a}$. Due to the explicit form of the super-operators (206), in the next section we will show that the above state is a Gaussian Fermionic state and that its two point correlation functions $\operatorname{Tr} \rho_{s}\left[w_{i}, w_{j}\right]$ are given by $\Gamma_{i j}$, i.e. by the solution of the continuous Lyapunov Eq. (214).

According to [390], the condition of uniqueness of the steady state is

$$
\Delta_{\mathcal{L}}:=2 \min _{j} \operatorname{Re}\left(x_{j}\right)>0,
$$

where the $x_{j}$ 's are the eigenvalues of $X$, and $\Delta_{\mathcal{L}}$ is the Liouvillian spectral gap. When this condition is met, any other state will eventually decay into the NESS in a time scale $\tau \sim 1 / \Delta_{\mathcal{L}}$. In the thermodynamic limit $n \rightarrow \infty$ a vanishing gap $\Delta(n) \rightarrow 0$ may be accompanied, though not necessarily, by non-differentiable properties of the NESS [123,365]. For this reason, the scaling of $\Delta_{\mathcal{L}}(n)$ has been used as an indicator of NESS criticality [364,365,381,393,394]. NESS-QPTs have been investigated through the scaling of the Bures metrics [42,165], whose super-extensivity has been connected to a vanishing $\Delta_{\mathcal{L}}$ [177]. Along the same line, it is also possible to demonstrate that a similar relation exists between the scaling properties of the dissipative gap and the mean Uhlmann curvature [110,113,118-120]. Essentially, one will develop in the context of NESS-QPTs an argument similar to the one established in Section 6.4, for the zero-temperature quantum phase transitions. There the expression (88) defines a necessary relation between the scaling of the quantum geometric tensor and a vanishing Hamiltonian gap. Here we will provide a relation connecting the scaling properties of the (generalised) quantum geometric tensor and the dissipative gap $\Delta_{\mathcal{L}}$.

\subsection{Diagonalisation of the Lindblad equation}

Following the notation introduced in Section 15, the Liouvillian (204) can be re-expressed as

$$
\mathcal{L}=-\frac{1}{2}\left(\begin{array}{ll}
\boldsymbol{a}^{\dagger} & \boldsymbol{a}
\end{array}\right)\left(\begin{array}{cc}
X & Y \\
0 & -X^{T}
\end{array}\right)\left(\begin{array}{c}
\boldsymbol{a} \\
\boldsymbol{a}^{\dagger}
\end{array}\right)-\frac{1}{2} \operatorname{Tr} X .
$$

Consider the following invertible transformation

$$
T:=\left(\begin{array}{ll}
\mathbb{1} & \Gamma \\
0 & \mathbb{1}
\end{array}\right), \quad T^{-1}:=\left(\begin{array}{cc}
\mathbb{1} & -\Gamma \\
0 & \mathbb{1}
\end{array}\right),
$$


If $\Gamma$ is the matrix solution of (214), then

$$
\left(\begin{array}{cc}
X & Y \\
0 & -X^{T}
\end{array}\right)=\hat{T}^{-1}\left(\begin{array}{cc}
X & Y-X \Gamma-\Gamma X^{T} \\
0 & -X^{T}
\end{array}\right) \hat{T}=\hat{T}^{-1}\left(\begin{array}{cc}
X & 0 \\
0 & -X^{\hat{T}}
\end{array}\right) \hat{T} .
$$

Therefore, one straightforwardly sees that the matrix (222) is diagonalised by the following transformation

$$
\hat{S}:=\left(\begin{array}{cc}
U^{-1} & 0 \\
0 & U^{T}
\end{array}\right) \hat{T}=\left(\begin{array}{cc}
U^{-1} & U^{-1} \Gamma \\
0 & U^{T}
\end{array}\right) .
$$

One can show that $\hat{S}$ is a non-unitary Bogoliubov transformation [392], which amounts to verify that $\hat{S}$ fulfils the following condition (see Eq. (2.6) of [392])

$$
\hat{S} \Sigma \hat{S}^{T}=\Sigma \quad \text { where } \quad \Sigma:=\left(\begin{array}{ll}
0 & \mathbb{1} \\
\mathbb{1} & 0
\end{array}\right) .
$$

This transformation leads to the definition of a new set of creation and annihilation super-operators as

$$
\left(\begin{array}{c}
\boldsymbol{b} \\
\boldsymbol{b}^{\times}
\end{array}\right)=\hat{S}\left(\begin{array}{c}
\boldsymbol{a} \\
\boldsymbol{a}^{\dagger}
\end{array}\right)
$$

Since $\mathcal{S}$ is a non-unitary Bogoliubov transformation, the operators $\hat{b}_{j}$ and $\hat{b}_{j}^{\times}$satisfy the canonical anti-commutation relations, but $\hat{b}_{j}^{\times} \neq \hat{b}_{j}^{\dagger}$. Moreover, by employing the relation $\left(\begin{array}{ll}\boldsymbol{a}^{\dagger} & \boldsymbol{a}\end{array}\right)=\left(\begin{array}{c}\boldsymbol{a} \\ \boldsymbol{a}^{\dagger}\end{array}\right)^{T} \Sigma^{x}$, together with the property (224), one finds that

$$
\mathcal{L}=-\frac{1}{2}\left(\begin{array}{ll}
\boldsymbol{b}^{\times} & \boldsymbol{b}
\end{array}\right)\left(\begin{array}{cc}
D_{X} & 0 \\
0 & -D_{X}
\end{array}\right)\left(\begin{array}{c}
\boldsymbol{b} \\
\boldsymbol{b}^{\times}
\end{array}\right)-\frac{1}{2} \operatorname{Tr} X,
$$

i.e.,

$$
\mathcal{L}=-\sum_{j} x_{j} \hat{b}_{j}^{\times} \hat{b}_{j}
$$

To the canonical transformation (225) it corresponds an operator acting on the Fock space, which, thanks to Eq. (2.16) of [392], can be written into the form

$$
\hat{b}_{j}=\mathcal{S} \hat{a}_{j} \mathcal{S}^{-1}, \quad \hat{b}_{j}^{\times}=\mathcal{S} \hat{a}_{j}^{\dagger} \mathcal{S}^{-1},
$$

where

$$
\mathcal{S}=: \exp \left(-\frac{1}{2} \boldsymbol{a}^{\dagger} \Gamma \boldsymbol{a}^{\dagger}+\boldsymbol{a}^{\dagger}(U-1) \boldsymbol{a}\right):
$$

and $: \exp (\cdot)$ : denotes the normal ordering of the exponential.

By exploiting the above operator, one is then able to explicitly express the vacuum of the Bogoliubov operators $\boldsymbol{b}$, i.e. the stationary state $\rho_{s}$ of the Liouvillian, in terms of the original super-operators $\boldsymbol{a}$. Recall that the vacuum of $\boldsymbol{a}$, i.e. the element $\mid \mathbf{0}) \in \mathcal{R}$ such that $\left.\hat{a}_{i} \mid \mathbf{0}\right)=0, \forall j=1, \ldots, 2 n$, is the completely mixed state. It also fulfils the property $(\mathbf{0} \mid \mathcal{L}=0$.

The vacuum of the Bogoliubov operators $\boldsymbol{b}$ can be readily obtained from the operator $\left.\mathcal{S}: \mid \rho)_{s}=\mathcal{S} \mid \mathbf{0}\right)$. Indeed, as $\left.\hat{a}_{j} \mid \mathbf{0}\right)=0$, one has $\left.\left.\hat{b}_{j} \mid \rho_{s}\right)=\mathcal{S} \hat{a}_{j} \mathcal{S}^{-1} \mathcal{S} \mid \mathbf{0}\right)=0$. Hence,

$$
\left.\left.\left.\mid \rho_{s}\right)=\mathcal{S} \mid \mathbf{0}\right)=e^{-\frac{1}{2} \boldsymbol{a}^{\dagger} \Gamma \boldsymbol{a}^{\dagger}} \mid \mathbf{0}\right) .
$$

The state (230) is exactly (201), as will be shown in the following. Thanks to the transformation $Q$ defined in (198), one can write $\Gamma$ in a canonical form with respect to the set of Fermion eigenmodes $\boldsymbol{z}$. By using the definition (230), one gets

$$
\frac{1}{2} \boldsymbol{a}^{\dagger} \Gamma \boldsymbol{a}^{\dagger} \rho=\frac{1}{8}(\boldsymbol{w} \cdot \Gamma \boldsymbol{w} \rho+2 \boldsymbol{w} \cdot \Gamma \rho \boldsymbol{w}+\rho \boldsymbol{w} \cdot \Gamma \boldsymbol{w})=\sum_{k} \mathcal{G}_{k}(\rho),
$$

where

$$
\mathcal{G}_{k}(\rho):=\frac{i}{4} \gamma_{k}\left[z_{2 k-1} z_{2 k} \rho+z_{2 k-1} \rho z_{2 k}-z_{2 k} \rho z_{2 k-1}+\rho z_{2 k-1} z_{2 k}\right] .
$$

One can verify the following two properties,

$$
\left\{\begin{array}{l}
\mathcal{G}_{k}(\mathbb{1})=i \gamma_{k} z_{2 k-1} z_{2 k}, \\
\mathcal{G}_{k}\left(z_{2 k-1} z_{2 k}\right)=0
\end{array}\right.
$$


which straightforwardly leads to

$$
\left.\rho_{s} \propto e^{-\frac{1}{2} \boldsymbol{a}^{\dagger} \Gamma \boldsymbol{a}^{\dagger}} \mid 0\right) \propto \prod_{k} e^{-\mathcal{G}_{k}} \mathbb{1}=\prod_{k}\left(1-i \gamma_{k} z_{2 k-1} z_{2 k}\right),
$$

thus recovering Eq. (201).

\subsection{Liouvillian spectrum}

The conditions for the existence and uniqueness of (234) have been derived in [390]. We now review those conditions and express them in terms of the spectral gap $\Delta_{\mathcal{L}}$.

The correlation matrix $\Gamma \in M_{2 n}(\mathbb{C})$ is the matrix solution of Eq. (214). This equation acquires a familiar linear matrix representation, when expressed through the so called (non-canonical) "vectorising" isomorphism

$$
\text { vec: } M_{2 n}(\mathbb{C}) \rightarrow\left(\mathbb{C}^{2 n}\right)^{\otimes 2} /|i\rangle\langle j|\rightarrow| i\rangle \otimes|j\rangle,
$$

which vectorises a matrix. This is also a Hilbert-space isomorphism, namely

$$
\langle\operatorname{vec}(A), \operatorname{vec}(B)\rangle=(A, B):=\operatorname{tr}\left(A^{\dagger} B\right) .
$$

One can directly check that

$$
\operatorname{vec}(A B C)=\left(A \otimes C^{T}\right) \operatorname{vec}(B) .
$$

Applying the vectorising isomorphism to both sides of continuous Lyapunov Eq. (214) one then gets

$$
\hat{X} \operatorname{vec}(\Gamma)=\operatorname{vec}(Y), \quad \hat{X}:=(X \otimes \mathbf{1}+\mathbf{1} \otimes X),
$$

where $\hat{X} \in \operatorname{End}\left(\mathbb{C}^{2 n}\right)^{\otimes 2} \cong M_{4 n^{2}}(\mathbb{C})$. There are three distinct operators involved in the above formalism, and correspondingly there are three different definitions of spectral gaps, which are described in the following.

1. The map $X: \mathbb{C}^{2 n} \rightarrow \mathbb{C}^{2 n}$, a $2 n \times 2 n$ real diagonalisable matrix. Its spectrum is $\left\{x_{j}\right\}_{j=1}^{2 n} \subset \mathbb{C}$ and, because of reality, is invariant under complex conjugation. From the non-negativity of the bath matrix $M$ one can prove that $\operatorname{Re} x_{j} \geq 0, \forall j$. (see Appendix B).

2. The map $\hat{X}=X \otimes \mathbf{1}+\mathbf{1} \otimes X \in \operatorname{End}\left(\mathbb{C}^{2 n}\right)^{\otimes 2} \cong M_{4 n^{2}}(\mathbb{C})$, a $4 n^{2} \times 4 n^{2}$ matrix. Since $X$ is assumed diagonalisable, also $\hat{X}$ will be so, and its spectrum is $\left\{x_{i}+x_{j}\right\}_{i, j=1}^{2 n} \subset \mathbb{C}$. The minimum of its modulus is clearly given by $\Delta_{\hat{X}}:=\min _{i, j}\left|x_{i}+x_{j}\right|$ Importantly, diagonalisability of $\hat{X}$ straightforwardly implies

$$
\Delta_{\hat{X}}^{-1}=\left\|\hat{X}^{-1}\right\|_{\infty}
$$

Moreover, for the uniqueness of the steady state we must have $\hat{X}$ invertible i.e., $\Delta_{\hat{X}}>0$.

3. The Liouvillian $\mathcal{L}$ : $\operatorname{End}\left(\left(\mathbb{C}^{2}\right)^{\otimes n}\right) \rightarrow \operatorname{End}\left(\left(\mathbb{C}^{2}\right)^{\otimes n}\right)$, a $2^{2 n} \times 2^{2 n}$ matrix. As it can be seen from Eq. (227), its spectrum can be defined through the array occupation numbers $\boldsymbol{n}:=\left(n_{1}, \ldots, n_{2 n}\right)^{T}$, where $n_{k}=0,1$ are the eigenvalues of Bogoliubov number operators $b_{k}^{\times} b_{k}$. Its spectrum is given by

$$
\operatorname{Sp}(\mathcal{L})=\left\{-x_{\boldsymbol{n}}:=\boldsymbol{n}^{T} \cdot \boldsymbol{x} \in \mathbb{C}\right\} \quad \text { where } \boldsymbol{x}:=\left(x_{1}, \ldots, x_{2 n}\right)^{T}, \text { with } x_{k} \in \operatorname{Sp}(X) .
$$

Notice that $0 \in \operatorname{Sp}(\mathcal{L})$ i.e. $\mathcal{L}$ is always non-invertible and the steady state (e.g., the Gaussian state $\boldsymbol{n}=\mathbf{0}$ ) is in the kernel of $\mathcal{L}$. If this latter is one-dimensional, i.e. a unique steady state, the gap of $\mathcal{L}$ can be defined as $\Delta_{\mathcal{L}}:=\min _{\mathbf{n} \neq \mathbf{0}}\left|x_{\mathbf{n}}\right|$.

Notice that, on account of the stability of the physical system, one is expected to have $\operatorname{Re} x_{j} \geq 0, \forall j$. Indeed, this is the quantum equivalent of the classical Lyapunov stability condition, where the time-scale for convergence $\rho(t) \rightarrow \rho(\infty)$ is dictated by $\tilde{\Delta}^{-1}$, with $\tilde{\Delta}=\min _{\mathbf{n} \neq \mathbf{0}} \boldsymbol{\operatorname { R e }} x_{\mathbf{n}}$.

It is not hard to show, that the three distinct definitions of spectral gaps just described effectively collapse into each other.

Proposition 1. If $\Delta=\min _{j} 2 \operatorname{Re}\left(x_{j}\right)>0$ then

$$
\Delta=\Delta_{\mathcal{L}}=\Delta_{\hat{X}} .
$$

Proof. $\left|x_{\mathbf{n}}\right|=\left|\boldsymbol{n}^{T} \cdot \boldsymbol{x}\right| \geq\left|\boldsymbol{R e}\left(\boldsymbol{n}^{T} \cdot \boldsymbol{x}\right)\right|$. The first bound can be saturated by choosing the $n_{j}$ 's in such a way that only a set $P$ of complex conjugated pairs $x_{p}^{ \pm}$of eigenvalues are present. In this case $\left|\boldsymbol{R e}\left(\boldsymbol{n}^{T} \cdot \boldsymbol{x}\right)\right|=2 \sum_{p \in P} \operatorname{Re} x_{p}$, where we used the assumption $(\forall p) \operatorname{Re} x_{p} \geq 0$. Using again positivity of all the terms, this sum can be made as small as possible by choosing $|P|=1$ and minimising over $p=1, \ldots, n$. This shows that $\Delta_{\mathcal{L}}=\min _{\mathbf{n}}\left|x_{\mathbf{n}}\right|=2 \min \left\{\boldsymbol{R e} x_{p}\right\}_{p=1}^{n}$. By a similar argument one shows that $\Delta_{\hat{X}}=\min \left\{\left|x_{i}+x_{j}\right|\right\}_{i, j=1}^{2 n}$ is given by the same expression i.e. $\Delta_{\mathcal{L}}=\Delta_{\hat{X}}$. Finally $\Delta=2 \min _{\mathbf{n}} \boldsymbol{\operatorname { R e }} x_{\mathbf{n}} \equiv 2 \tilde{\Delta}=2 \min _{p} \boldsymbol{\operatorname { R e }} x_{p}=\Delta_{\mathcal{L}}$. 


\section{Geometric properties of the steady states}

We would like to transfer to the framework of NESS-QPTs the insight that we have learnt from the geometric information approach and the geometric phase methods that so far have been applied to the equilibrium case. The idea would be to explore the properties of the metrics and the properties of the geometric phases pretty much in the spirit of the equilibrium phase transitions. The natural candidate for the definition of a metric is clearly the Bures metric, as the intuition built from QPTs in open systems would suggest [165,199,214,214]. This has been done in Ref. [177].

A completely different story applies to the geometric phase, as a natural candidate in the mixed state domain does not exist. In the context of mixed quantum states, it is necessary to exploit unorthodox concepts of geometric phases and many possible definitions of the mixed state geometric phase have been put forward $[156,168-174,395,396]$. Which definition is best suited in this context depends largely on the type of information that one wants to pursue. In this context the Uhlmann GP [156] stands out for its deep rooted relations with the fidelity approach and for its relations with quantum estimation theory.

Motivated by this, we will concentrate on the mean Uhlmann curvature, which has been already introduced in Section 9. Rather than exploring the geometric phase itself, which provides insight on the global properties of the mixed state manifold, one can look at the Uhlmann curvature, as it conveys information on the local geometric structure of the parametric manifold. This choice is ideally suited to the study of non-equilibrium phase transitions which are related to local differential properties of the state space.

The mathematical properties of the MUC makes it an ideal candidate both at the conceptual and at the technical level. From a physical point of view the mean Uhlmann curvature is gauge invariant, thus ensuring that its behaviour is physically relevant and cannot emerge as an artefact of the gauge choice. Moreover, technically the MUC is much easier to handle than the full Uhlmann curvature, due to its scalar nature as opposed to the non-Abelian structure of the curvature.

We will review the general formulas which unify, within the same framework, Bures metrics and mean Uhlmann curvature over the set of Gaussian Fermionic states. This will be needed in order to discuss in the following sections how the scaling of the metric and the curvature provide information on the closing of $\Delta_{\mathcal{L}}$ and the divergences of the two-point correlations. Finally one can see this in action, by applying such a theoretical framework to exactly solvable models. This demonstrates that NESS phase diagram can be accurately mapped by studying the scaling behaviour and the singularities of the metric tensor $g$ and of the $\mathcal{U}$ : critical lines can be identified and the different phases distinguished.

Moreover, with joint information of both $g$ and $\mathcal{U}$ and from insight derived from quantum estimation theory, a concept of "quantumness" of the NESS-QPTs can be introduced. The aim of this approach is to glean an insight into the character of the fluctuations driving the non-equilibrium phase transitions.

The calculation of both Uhlmann curvature and Bures metrics in large Hilbert spaces is quite a daunting task. Standard approaches [157] are computationally not viable in many-body setups, where the quest for effective methods to evaluate both metric and curvature is the subject of active research [397]. We will show that in the physically relevant class of Gaussian Fermionic states this can be accomplished in a surprisingly efficient way [113].

\subsection{Mean Uhlmann curvature and Bures metric of Gaussian Fermionic states}

Before discussing the geometric properties of Gaussian Fermionic states, let us recall some basic properties of the correlation function. For GFS, all odd-order correlation functions are zero, and all even-order correlations, higher than two, can be obtained from covariance matrix $\Gamma$ by Wick's theorem [388], i.e.

$$
\operatorname{Tr}\left(\rho \omega_{k_{1}} \omega_{k_{2}} \ldots \omega_{k_{2 p}}\right)=\operatorname{Pf}\left(\Gamma_{k_{1} k_{2} \ldots k_{2 p}}\right), \quad 1 \leq k_{1}<\ldots<k_{2 p} \leq 2 n .
$$

$\Gamma_{k_{1} k_{2} \ldots k_{2 p}}$ is the corresponding $2 p \times 2 p$ submatrix of $\Gamma$ and $\operatorname{Pf}\left(\Gamma_{k_{1} k_{2} \ldots k_{2 p}}\right)^{2}=\operatorname{det}\left(\Gamma_{k_{1} k_{2} \ldots k_{2 p}}\right)$ is the Pfaffian. An especially useful case is the four-point correlation function

$$
\operatorname{Tr}\left(\rho \omega_{j} \omega_{k} \omega_{l} \omega_{m}\right)=a_{j k} a_{l m}-a_{j l} a_{k m}+a_{j m} a_{k l},
$$

where $a_{j k}:=\Gamma_{j k}+\delta_{j k}$.

To derive a convenient expression for the Uhlmann curvature and the Bures metric for Gaussian Fermionic states, first let us recall their expression in terms of the parallel transport generator (130):

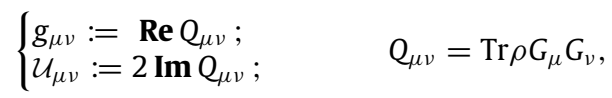

where $Q_{\mu v}$ is the generalised quantum geometric tensor (QGT) (see Eq. (154)).

The starting point is to derive the generator $\boldsymbol{G}$ in terms of the two point correlation matrix $\Gamma$. Due to the quadratic dependence of the density matrix on $\boldsymbol{\omega}$ (see Eq. (201)), and following the arguments of [113,398,399], it can be shown that $\boldsymbol{G}$ is a quadratic polynomial in the Majorana Fermions

$$
\boldsymbol{G}=: \frac{1}{4} \boldsymbol{\omega}^{T} \cdot \mathbf{K} \boldsymbol{\omega}+\zeta^{T} \boldsymbol{\omega}+\eta,
$$


where $\boldsymbol{K}=\sum_{\mu} K_{\mu} d \lambda_{\mu}$, and $K_{\mu}:=\left\{K_{\mu}^{j k}\right\}_{j k=1}^{2 n}$ are a set of $2 n \times 2 n$ Hermitian anti-symmetric matrices, $\zeta=\zeta_{\mu} d \lambda_{\mu}$, with $\zeta_{\mu}$ a $2 n$ real vector, and $\eta=\eta_{\mu} d \lambda_{\mu}$ a real valued one-form. Note that any odd-order correlation function for a Gaussian Fermionic state vanishes identically, then

$$
\left\langle\omega_{k}\right\rangle=\operatorname{Tr}\left(\rho \omega_{k}\right)=0 \quad \forall k=1 \ldots 2 n .
$$

By differentiating the above equation, one readily shows that the linear term in (245) is identically zero

$$
0=\operatorname{Tr}\left(\omega_{k} d \rho\right)=\operatorname{Tr}\left(\omega_{k}\{\boldsymbol{G}, \rho\}\right)=\operatorname{Tr}\left(\rho\left\{\zeta^{T} \boldsymbol{\omega}, \omega_{k}\right\}\right)=\zeta^{k},
$$

where $\zeta^{k}$ is the $k$ th component of $\zeta$, and in the third equality one takes into account that the third order correlations vanish. The quantity $\eta$ can be determined from the trace preserving condition, i.e.

$$
d \operatorname{Tr} \rho=\operatorname{Tr}(d \rho)=2 \operatorname{Tr}(\rho \boldsymbol{G})=0,
$$

which, after plugging in Eq. (245), leads to

$$
\eta=-\frac{1}{4} \operatorname{Tr}\left(\rho \boldsymbol{\omega}^{T} \boldsymbol{K} \boldsymbol{\omega}\right)=\frac{1}{4} \operatorname{Tr}(\boldsymbol{K} \Gamma) \text {. }
$$

In order to determine $\boldsymbol{K}$, let us take the differential of $\Gamma_{j k}=1 / 2 \operatorname{Tr}\left(\rho\left[\omega_{j}, \omega_{k}\right]\right)$, then

$$
\begin{aligned}
d \Gamma_{j k}=\frac{1}{2} \operatorname{Tr}\left(d \rho\left[\omega_{j}, \omega_{k}\right]\right) & =\frac{1}{2} \operatorname{Tr}\left(\{\rho, \boldsymbol{G}\}\left[\omega_{j}, \omega_{k}\right]\right) \\
& =\frac{1}{8} \operatorname{Tr}\left(\left\{\rho, \boldsymbol{\omega}^{T} \boldsymbol{K} \boldsymbol{\omega}\right\}\left[\omega_{j}, \omega_{k}\right]\right)+\boldsymbol{\eta} \frac{1}{2} \operatorname{Tr}\left(\rho\left[\omega_{j}, \omega_{k}\right]\right) \\
& =\frac{1}{8} \sum_{l m} K^{l m} \operatorname{Tr}\left(\left\{\rho,\left[\omega_{l}, \omega_{m}\right]\right\}\left[\omega_{j}, \omega_{k}\right]\right)+\eta \Gamma_{j k} \\
& =(\Gamma \boldsymbol{K} \Gamma-\boldsymbol{K})_{j k}+\left[\boldsymbol{\eta}-\frac{1}{4} \operatorname{Tr}(\boldsymbol{K} \Gamma)\right] \Gamma_{j k},
\end{aligned}
$$

where the last equality is obtained with the help of Eq. (243) and using the antisymmetry of $\Gamma$ and $\boldsymbol{K}$ under the exchange of $j$ and $k$. Finally, according to Eq. (248), the last term vanishes and we obtain the following (discrete time) Lyapunov equation

$$
d \Gamma=\Gamma \boldsymbol{K} \Gamma-\boldsymbol{K} .
$$

The above equation can be formally solved by

$$
\boldsymbol{K}=\left(\operatorname{Ad}_{\Gamma}-\mathbb{1}\right)^{-1}(d \Gamma),
$$

where $\operatorname{Ad}_{\Gamma}(X):=\Gamma X \Gamma^{\dagger}$ is the adjoint action. In the eigenbasis of $\Gamma$ (i.e. $\Gamma|j\rangle=\gamma_{j}|j\rangle$ ) it reads

$$
\langle j|\boldsymbol{K}| k\rangle=(\boldsymbol{K})_{j k}=\frac{(d \Gamma)_{j k}}{\gamma_{j} \gamma_{k}-1}=-\frac{d \Omega_{k}}{2} \delta_{j k}+\tanh \frac{\Omega_{j}-\Omega_{k}}{2}\langle j \mid d k\rangle,
$$

where, in the second equality, we made use of the relation $\gamma_{k}=\tanh \left(\Omega_{k} / 2\right)$, which yields the following diagonal $(d \Gamma)_{j j}=\left(1-\gamma_{j}^{2}\right) d \Omega_{j}$ and off-diagonal terms $(d \Gamma)_{j k}=\left(\gamma_{k}-\gamma_{j}\right)\langle j \mid d k\rangle$. This expression is well defined everywhere except for $\gamma_{j}=\gamma_{k}= \pm 1$, where the Gaussian state $\rho$ becomes singular, i.e. it is not full rank. In this condition, the expression (251) for the generator $\boldsymbol{G}$ may become singular. Nevertheless, the boundness of the function $\left|\tanh \frac{\Omega_{j}-\Omega_{k}}{2}\right| \leq 1$ in Eq. (251) shows that such a singularity is relatively benign. Thanks to this, we can show that the condition $\gamma_{j}=\gamma_{k}= \pm 1$ produces, at most, removable singularities in the QGT (see. [289]). This allows the quantum geometric tensor to be extended by continuity from the set of full-rank density matrices to the submanifolds with $\gamma_{j}=\gamma_{k}= \pm 1$.

Knowing the expression for the parallel transport generator $\boldsymbol{G}$, we can calculate the QGT by plugging $G_{\mu}=\frac{1}{4}\left[\omega^{T} K_{\mu} \omega-\right.$ $\left.\operatorname{Tr}\left(K_{\mu} \cdot \Gamma\right)\right]$ into $Q_{\mu \nu}:=\operatorname{Tr}\left(\rho G_{\mu} G_{\nu}\right)$. Making use of Eq. (243) and exploiting the antisymmetry of both $\Gamma$ and $\boldsymbol{K}$ under the exchange of Majorana Fermion indices leads to [110]

$$
\begin{aligned}
Q_{\mu \nu} & =\frac{1}{8} \operatorname{Tr}\left[(\mathbb{1}-\Gamma) K_{\mu}(\mathbb{1}+\Gamma) K_{v}\right] \\
& =\frac{1}{8} \sum_{j k}\left(1-\gamma_{j}\right)\left(1+\gamma_{k}\right) K_{\mu}^{j k} K_{\nu}^{k j} \\
& =\frac{1}{8} \sum_{j k} \frac{\left(1-\gamma_{j}\right)\left(1+\gamma_{k}\right)}{\left(1-\gamma_{j} \gamma_{k}\right)^{2}}\left(\partial_{\mu} \Gamma\right)_{j k}\left(\partial_{\nu} \Gamma\right)_{k j},
\end{aligned}
$$

where the last equality is obtained by plugging in Eq. (251). Let us have a closer look at the QGT in the limit of $\left(\gamma_{j}, \gamma_{k}\right) \rightarrow \pm(1,1)$. The boundness of $\boldsymbol{K}_{j k}$ and the multiplicative factors $\left(1 \pm \gamma_{j}\right)$ in Eq. (252) cause each term to vanish with 
$\left|\gamma_{j}\right| \rightarrow 1$. This means that the QGT has a well defined value in the above limit, and we can safely extend by continuity the QGT to the sub-manifolds $\left(\gamma_{j}, \gamma_{k}\right)= \pm(1,1)$.

The explicit expression of $Q_{\mu \nu}$ produces the following results for the Bures metrics

$$
\begin{aligned}
g_{\mu \nu} & =\operatorname{Re}\left(Q_{\mu \nu}\right)=\frac{1}{8} \operatorname{Tr}\left(K_{\mu} K_{v}-\Gamma K_{\mu} \Gamma K_{v}\right) \\
& =-\frac{1}{8} \operatorname{Tr}\left(\partial_{\mu} \Gamma K_{v}\right) \\
& =\frac{1}{8} \sum_{j k} \frac{\left(\partial_{\mu} \Gamma\right)_{j k}\left(\partial_{\nu} \Gamma\right)_{k j}}{1-\gamma_{j} \gamma_{k}},
\end{aligned}
$$

which in a parameter independent way reads

$$
d l^{2}=\sum_{\mu \nu} g_{\mu \nu} d \lambda_{\mu} d \lambda_{\nu}=\frac{1}{8} \operatorname{Tr}\left[d \Gamma \frac{1}{\mathbb{1}-\operatorname{Ad}_{\Gamma}}(d \Gamma)\right],
$$

where $d \Gamma:=\sum_{\mu} \partial_{\mu} \Gamma d \lambda_{\mu}$. Eq. (253) has been obtained by substituting the formal solution (250) of $\boldsymbol{K}$ in the second equality of Eq. (253). The above expression was derived by a different procedure by Banchi et al. [177]. For the MUC the explicit expression is

$$
\begin{aligned}
\mathcal{U}_{\mu \nu} & =2 \operatorname{Im}\left(Q_{\mu \nu}\right)=\frac{i}{4} \operatorname{Tr}\left(\Gamma\left[K_{\mu}, K_{\nu}\right]\right) \\
& =\frac{i}{4} \sum_{j k} \frac{\gamma_{k}-\gamma_{j}}{\left(1-\gamma_{j} \gamma_{k}\right)^{2}}\left(\partial_{\mu} \Gamma\right)_{j k}\left(\partial_{\nu} \Gamma\right)_{k j} .
\end{aligned}
$$

Also the above expression can be cast in a parameter-independent way. Exploiting Eq. (250) leads to

$$
\begin{aligned}
\mathcal{U} & =\frac{i}{4} \operatorname{Tr}(\Gamma \boldsymbol{K} \wedge \boldsymbol{K}) \\
& =\frac{i}{4} \operatorname{Tr}\left[\Gamma \frac{1}{\mathbb{1}-\operatorname{Ad}_{\Gamma}}(d \Gamma) \wedge \frac{1}{\mathbb{1}-\operatorname{Ad}_{\Gamma}}(d \Gamma)\right] .
\end{aligned}
$$

Eq. (253) reproduces known results for the thermal states [165] and for pure states $[198,199]$. On the other hand, no previous account of a close form expression of the Uhlmann curvature were known in literature for the case of Fermionic Gaussian states, they being in the equilibrium or out of equilibrium condition. As expected, formula (254) reduces to the correct expression in the case of pure states, provided that the appropriate matrix $\Gamma$ is considered $[31,32,37,39]$.

\subsection{Super-extensivity of the (generalised) quantum geometric tensor}

The above results apply to the general class of Gaussian Fermionic states. In this section, we will derive some results which are specific to the quadratic Liouvillian models considered. The aim of this section is to connect the kinematic properties embodied by the quantum geometric tensor to the dynamical features of the underlying physical model. More specifically we will derive a bound similar to the one obtained in Section 6.4, which relates the super-extensivity of the generalised quantum geometric tensor to the dissipative gap.

As in the case of Eq. (68) also $Q(\lambda)$ defined in Eq. (154) is a Hermitian non-negative matrix. Thus one has

$$
\left|Q_{\mu \nu}\right| \leq\|Q\|_{\infty}=\boldsymbol{u}^{\dagger} \cdot Q \cdot \boldsymbol{u},
$$

where $\boldsymbol{u}=\left\{u_{\mu}\right\}_{\mu=1}^{\operatorname{dim} \mathcal{M}}$, with $\boldsymbol{u}^{\dagger} \cdot \boldsymbol{u}=1$, is the normalised eigenvector of $Q$ with the largest eigenvalue. One can define the corresponding combination of parameters $\bar{\lambda}:=\sum_{\mu=1}^{\operatorname{dim} \mathcal{M}} u_{\mu} \lambda_{\mu}$ and the corresponding directional derivative $\bar{\partial}:=\partial / \partial \bar{\lambda}$, so that

$$
\bar{\partial} \Gamma=\sum_{\mu}\left(\partial_{\mu} \Gamma\right) u_{\mu}
$$

and from Eq. (80) and the above inequality (255) we get

$$
\|Q\|_{\infty}=\frac{1}{8} \operatorname{Tr}\left[(\mathbb{1}-\Gamma) \frac{1}{\mathbb{1}-\operatorname{Ad}_{\Gamma}}(\bar{\partial} \Gamma)(\mathbb{1}+\Gamma) \frac{1}{\mathbb{1}-\operatorname{Ad}_{\Gamma}}(\bar{\partial} \Gamma)\right] .
$$

Let us express Eq. (257) in a form amenable to further manipulations, by employing the vectorisation isomorphism. Notice that, under such an isomorphism

$$
\operatorname{Ad}_{\Gamma}(A):=\Gamma A \Gamma^{\dagger} \stackrel{\text { vec }}{\longrightarrow}\left(\Gamma \otimes \Gamma^{T}\right) \operatorname{vec}(A)=-(\Gamma \otimes \Gamma) \mathbf{v e c}(A),
$$


and Eq. (257) becomes

$$
\begin{aligned}
\|Q\|_{\infty} & =\frac{1}{8} \operatorname{vec}(\bar{\partial} \Gamma)^{\dagger} \cdot\left(\frac{(\mathbb{1}+\Gamma) \otimes(\mathbb{1}+\Gamma)}{\mathbb{1}+\Gamma \otimes \Gamma}\right) \cdot \operatorname{vec}(\bar{\partial} \Gamma) \\
& \leq P_{\Gamma}\|\operatorname{vec}(\bar{\partial} \Gamma)\|^{2} \\
& \leq 2 n P_{\Gamma}\|\bar{\partial} \Gamma\|_{\infty}^{2},
\end{aligned}
$$

where

$$
P_{\Gamma}:=\frac{1}{8}\left\|\frac{(\mathbb{1}+\Gamma) \otimes(\mathbb{1}+\Gamma)}{\mathbb{1}+\Gamma \otimes \Gamma}\right\| .
$$

In the first inequality of Eq. (258), we used the definition of operator norm, while in the second we have employed the fact that $\|\operatorname{vec}(A)\|=\|A\|_{2}$ and $\|A\|_{2} \leq \sqrt{2 n}\|A\|_{\infty}$.

The bound (258) still is not specific to dissipative quadratic Liouvillian. In order to relate Eq. (258) to the dynamical properties of the Liouvillian (227) one could differentiate Eq. (238)

$$
\operatorname{vec}\left(\partial_{\mu} \Gamma\right)=\hat{X}^{-1} \operatorname{vec}\left(\partial_{\mu} Y\right)-\hat{X}^{-1} \partial_{\mu} \hat{X} \mathbf{v e c}(\Gamma) .
$$

Through the above equation, one realises that $\partial_{\mu} \Gamma$ is the solution of a continuous Lyapunov equation, similar to (214), which provides a convenient way to calculate it numerically once $\Gamma, X, Y$ and $\partial_{\mu} X$ are known, i.e.

$$
X\left(\partial_{\mu} \Gamma\right)+\left(\partial_{\mu} \Gamma\right) X^{T}=\partial_{\mu} Y-\left(\partial_{\mu} X\right) \Gamma-\Gamma\left(\partial_{\mu} X^{T}\right) .
$$

Taking norms in Eq. (260) leads to ${ }^{5}$

$$
\begin{aligned}
\left\|\partial_{\mu} \operatorname{vec}(\Gamma)\right\| & \leq\left\|\hat{X}^{-1}\right\|_{\infty}\left(\left\|\partial_{\mu} \operatorname{vec}(Y)\right\|+\left\|\partial_{\mu} \hat{X}\right\|_{\infty}\|\operatorname{vec}(\Gamma)\|\right) \\
& =\left\|\hat{X}^{-1}\right\|_{\infty}\left(\left\|\partial_{\mu} Y\right\|_{2}+\left\|\partial_{\mu} \hat{X}\right\|_{\infty}\|\Gamma\|_{2}\right) \\
& \leq \sqrt{2 n}\left\|\hat{X}^{-1}\right\|_{\infty}\left(\left\|\partial_{\mu} Y\right\|_{\infty}+\left\|\partial_{\mu} \hat{X}\right\|_{\infty}\|\Gamma\|_{\infty}\right) \\
& \leq \sqrt{2 n}\left\|\hat{X}^{-1}\right\|_{\infty}\left(\left\|\partial_{\mu} Y\right\|_{\infty}+\left\|\partial_{\mu} \hat{X}\right\|_{\infty}\right),
\end{aligned}
$$

where relations $\|\operatorname{vec}(A)\|=\|A\|_{2},\|A\|_{2} \leq \sqrt{2 n}\|A\|_{\infty}$ and $\|\Gamma\|_{\infty} \leq 1$ have been employed, the latter following from Eq. (199).

Essentially, the upper bound for $\partial_{\mu} \Gamma$, obtained above, only depends on the system parameters and their differentials, i.e., $X, d X$ and $Y, d Y$. Finally one derives the following bound

$$
\left\|\partial_{\mu} \mathbf{v e c}(\Gamma)\right\|^{2} \leq 2 n\left\|\hat{X}^{-1}\right\|_{\infty}^{2}\left(\left\|\partial_{\mu} Y\right\|_{\infty}+2\left\|\partial_{\mu} X\right\|_{\infty}\right)^{2}
$$

where the relation $\left\|\partial_{\mu} \hat{X}\right\|_{\infty}=\left\|\partial_{\mu} X \otimes \mathbf{1}+\mathbf{1} \otimes \partial_{\mu} X\right\|_{\infty} \leq 2\left\|\partial_{\mu} X\right\|_{\infty}$ has been used.

Now, we finally wrap all the latest results around: by plugging Eq. (263) in (257) and by employing relation (239) and Proposition 1 of Section 15.2, one eventually obtains the following upper bound which relates the behaviour of $\Delta(n)$ to $\left|Q_{\mu \nu}\right|$, i.e.

$$
\frac{\left|Q_{\mu \nu}\right|}{n} \leq 2 \frac{P_{\Gamma}}{\Delta_{\mathcal{L}}^{2}}\left(\|d Y\|_{\infty}+2\|d X\|_{\infty}\right)^{2} .
$$

The latter is the relation that was anticipated earlier: it is the dissipative analogue of the inequality for zero-temperature QPT derived in Section 6.4, where it was shown that super-extensivity of the quantum geometric tensor $Q_{\mu \nu}$ implies the vanishing of the energy gap [42] and the outset of a phase transitions. The above bound connects the generalised QGT to the dynamical feature of the dissipative Liouvillian model. It is indeed a relation between the kinematics expressed by the geometry of the NESS and the dynamics, embodied by the dissipative gap. Specifically, this bound shows that, if $P_{\Gamma} \simeq \mathcal{O}(1)$, a scaling of $\left|Q_{\mu \nu}\right| \propto n^{\alpha+1}$ entails a dissipative gap that vanishes at least as $\Delta_{\mathcal{L}} \propto n^{-\alpha / 2}$, establishing a link between the dynamical properties of the NESS-QPTs and the geometric property $Q_{\mu \nu}$.

Needless to say, the above bound on the QGT immediately determines bounds on both the Bures metric and on the mean Uhlmann geometric phase,

$$
\begin{aligned}
& \left|g_{\mu \nu}\right|=\left|\mathbf{R e} Q_{\mu \nu}\right| \leq\left|Q_{\mu \nu}\right|, \\
& \left|\mathcal{U}_{\mu \nu}\right|=\left|\mathbf{I m} Q_{\mu \nu}\right| \leq\left|Q_{\mu \nu}\right|,
\end{aligned}
$$

whose scaling properties can thus be related to the NESS-QPTs.

It is important, however, to stress that $\Delta_{\mathcal{L}}$ is an entirely different quantity from the Hamiltonian gap, linked to the scaling of $Q_{\mu \nu}$ for zero-temperature-QPTs. A complete understanding of the relation between the Liouvillian gap

$5\|O\|_{\infty}:=\sup _{\|v\|=1}\|O v\|=$ largest singular value of $O$; Notice that $\|O v\| \leq\|O\|_{\infty}\|v\| .\|O\|_{2}^{2}=\operatorname{Tr}\left(O^{\dagger} O\right)=$ sum of the squares of the singular values of 0 . 
and the Hamiltonian gap ruling equilibrium QPTs is still lacking. Notice, indeed, that in the non-dissipative case the spectrum $\mathrm{Sp}(X)$ is purely imaginary. From the perspective of the Liouvillian dynamics, this implies an identically vanishing dissipative gap $\Delta_{\mathcal{L}} \equiv 0$. This contrasts with the naïve attempt of formulating a general equilibrium/non-equilibrium QPT criterion which levels the dissipative gap to the same status of a Hamiltonian gap in standard QPTs. Moreover, unlike in equilibrium QPTs [268], where super-extensivity is a sufficient condition for criticality at $T=0$, in the dissipative case $\left|Q_{\mu \nu}\right|=\mathcal{O}\left(n^{1+\alpha}\right)(\alpha>0)$ only implies $\Delta_{\mathcal{L}}=\mathcal{O}\left(n^{\alpha / 2}\right)$, but it does not necessarily imply criticality. Indeed, in NESS-QPT, a closure of the gap generally neither is implied by criticality nor implies it $[110,123]$.

On the other hand, one can see that in the case of translationally invariant models, where a notion of criticality in the thermodynamic limit is easier to handle [191,380], further progress can be done. There, the problem of relating the geometric properties to the dynamical features of the model can be bypassed, in favour of a direct relation between the geometry and the divergence of the correlation length [110].

Note that in the non-diagonalisable case a correction to Eq. (239) should be considered, which adds an extra polynomial dependence in (241) [177,390]. However, this variation does not affect the qualitative and quantitative consequences of the bound (264): super-extensivity of the quantum geometric tensor entails a vanishing Liouvillian gap.

\section{Translationally invariant models}

Before turning to specific models where the above general considerations can be exemplified, we would like to draw the attention to an important subclass of quadratic Liouvillian Fermionic models, namely those enjoying the translational invariance symmetry. In a translationally invariant system one can employ the whole wealth of powerful tools stemming out of the Fourier transform and work directly in the thermodynamic limit. This enables one to quantitatively define criticality in terms of singularities in the quasi-momentum space, thereby secluding the kinematics of the NESS-QPTs from the dynamical properties of the model. The most natural notion of many-body criticality is in terms of diverging correlation length, which in a translationally invariant system is relatively straightforward to handle. This way of defining criticality enables one to bypass the difficulties arising from the ambiguous relation between NESS-QPTs and the vanishing dissipative gap.

The object of investigation is the covariance matrix, which in a translationally invariant system can be conveniently studied through its Fourier components. It is the non-analytical behaviour in the Fourier basis which conveys information on the long-wavelength limit, i.e. on the divergence of the correlation length.

Consider an explicit translationally invariant $d$-dimensional lattice of Fermions located at sites $r \in \mathbb{Z}_{L}^{d}$, and assume finite (or quasi-finite) range interaction. The system size is $n=L^{d}$ and, subsequently, one takes the thermodynamic limit $L \rightarrow \infty$. One can define the covariance matrix over a discrete quasi-momentum space. However, the considerations on the long-wavelength limit, that will follow, truly, apply only at the thermodynamic limit: hence divergences of correlation lengths manifest genuine quantum many-body effects.

To emphasise the translational property, let us relabel the Majorana Fermions as

$$
\boldsymbol{\omega}_{r}=\left(\begin{array}{l}
\omega_{r, 1} \\
\omega_{r, 2}
\end{array}\right), \quad \text { with }\left\{\begin{array}{l}
\omega_{r, 1}=c_{r}+c_{r}^{\dagger} \\
\omega_{r, 2}=i\left(c_{r}-c_{r}^{\dagger}\right)
\end{array}\right.
$$

where $\omega_{r, \beta},(\beta=1,2)$ are the two flavours of Majorana Fermions on each site $r$, and $c_{r}$ and $c_{r}^{\dagger}$ are the annihilation and creation operator, respectively, of the corresponding ordinary Fermion. Due to translational invariance, the Hamiltonian may be written as

$$
\mathcal{H}=\sum_{r, s} \omega_{r}^{T} h(r-s) \omega_{s}
$$

where $h(r)=h(-r)^{\dagger}=h(r)^{*}$ are $2 \times 2$ imaginary matrices. Similarly the jump operators can be expressed as

$$
\Lambda_{\alpha}(r)=\sum_{s} \boldsymbol{l}_{\alpha}^{T}(s-r) \boldsymbol{\omega}_{s}
$$

where $\boldsymbol{l}_{\alpha}(r)$ are 2-dimensional complex arrays. Accordingly, the bath matrix are written as

$$
[M]_{(r, \beta)\left(s, \beta^{\prime}\right)}=[m(r-s)]_{\beta \beta^{\prime}} \quad\left(\beta, \beta^{\prime}=1,2\right),
$$

where $m(r)=m^{\dagger}(-r)$ are the $2 \times 2$ matrices $m(r):=\sum_{\alpha, s} \boldsymbol{l}_{\alpha}(s-r) \otimes \boldsymbol{l}_{\alpha}^{\dagger}(s)$.

Since both Hamiltonian and bath matrix are circulant, so it is the correlation matrix of the unique steady state solution, which reads

$$
[\Gamma]_{(r, \beta)\left(s, \beta^{\prime}\right)}=[\gamma(r-s)]_{\beta \beta^{\prime}}:=\frac{1}{2} \operatorname{Tr}\left(\rho\left[\omega_{r, \beta}, \omega_{s, \beta^{\prime}}\right]\right), \quad\left(\beta, \beta^{\prime}=1,2\right) .
$$

The latter can be conveniently expressed in terms of its Fourier component, called the covariance symbol, as

$$
\tilde{\gamma}(\phi):=\sum_{r} \gamma(r) e^{-i \phi \cdot r}
$$


where $\phi \in[-\pi, \pi)$. In terms of the symbol functions, the continuous Lyapunov equation reduces to a set of $2 \times 2$ matrix equations

$$
\tilde{x}(\phi) \tilde{\gamma}(\phi)+\tilde{\gamma}(\phi) \tilde{x}^{T}(-\phi)=\tilde{y}(\phi),
$$

where $\tilde{x}(\phi)=2\left[2 i \tilde{h}(\phi)+\tilde{m}(\phi)+\tilde{m}^{T}(-\phi)\right]$ and $\tilde{y}(\phi)=-4\left[\tilde{m}(\phi)-\tilde{m}^{T}(-\phi)\right]$ are the symbol functions of $X$ and $Y$, respectively, and $\tilde{h}(\phi), \tilde{m}(\phi)=\sum_{\alpha} \tilde{\boldsymbol{l}}_{\alpha} \otimes \tilde{\boldsymbol{l}}_{\alpha}^{\dagger}$ and $\tilde{\boldsymbol{l}}_{\alpha}(\phi)$ are the Fourier components of $h(r), m(r)$ and $\boldsymbol{l}_{\alpha}(r)$, respectively. Notice that $\tilde{m}(\phi)=\tilde{m}(\phi)^{\dagger}=\sum_{\alpha} \tilde{\boldsymbol{l}}_{\alpha} \otimes \tilde{\boldsymbol{l}}_{\alpha}^{\dagger} \geq 0$ (positive semidefinite matrix).

The spatial correlations between Majorana Fermions are then recovered from the inverse Fourier transform of the covariance symbol

$$
\gamma(r)=\frac{1}{(2 \pi)^{d}} \int_{\mathbb{T}^{d}} d^{d} \phi \tilde{\gamma}(\phi) e^{i \phi \cdot r} .
$$

Following [191,380], here we will define criticality by the divergence of correlation length, which is defined as

$$
\xi^{-1}:=-\lim _{|r| \rightarrow \infty} \frac{\ln \|\gamma(r)\|}{|r|} .
$$

In the thermodynamic limit, the divergence may only arise as a consequence of the non-analytical dependence of $\gamma(r)$ on the system parameters. Let us confine ourselves to the case of a one-dimensional Fermionic chain. In order to derive informations on the large distance behaviour of the correlations, it is convenient to express the above integral (273) in the complex plane through the analytical continuation $e^{i \phi} \rightarrow z$. This results in the following expression for the correlation function

$$
\gamma(r)=\sum_{\bar{z} \in S_{1}} \operatorname{Res}_{\bar{z}}\left[z^{r-1} \tilde{\gamma}(z)\right]
$$

where $\operatorname{Res}_{\bar{z}}$ indicates the residues of the poles inside the unit circle $S_{1}:=\{z$, such that $|z| \leq 1\}$. Since $\tilde{\gamma}(z)$ is the solution of a finite dimensional matrix, i.e. Eq. (272), it may only possess simple poles. Thus, the above expression may become singular only when an isolated pole of $\tilde{\gamma}(z)$ approaches the unit circle from the inside [191,380]. This may happen for some specific critical values $\lambda=\lambda_{0} \in \mathcal{M}$. As $\lambda$ approaches $\lambda_{0}$ the correlation length $\xi$ diverges. One can show that the long wave-length behaviour is governed by the pole closest to the unit circle $\left|\bar{z}_{0}\right|$, and indeed the correlation length is given by

$$
\xi=\ln \left|\bar{z}_{0}\right| .
$$

\subsection{Mean uhlmann curvature and criticality in translationally invariant models}

Let us now turn to the geometric properties of translationally invariant models at criticality. In particular let us concentrate on the mean Uhlmann curvature. We will show that the MUC is sensitive to the criticality, but only in the sense of a truly diverging correlation length. Indeed one can show that the Uhlmann curvature is insensitive to the vanishing of the dissipative gap, if the latter, as it may happen, is not accompanied by a diverging correlation length. In this sense, the Uhlmann curvature confirms its role as a witness of the purely kinematic aspects of the criticality, and it is only indirectly affected by the dynamical features of the NESS-QPTs.

Thanks to the translational symmetry, one can exploit the formalism of Fourier transform and derive a quite compact expression of the MUC. By applying the convolution theorem on Eq. (254), one obtains the following expression for the MUC per site

$$
\overline{\mathcal{U}}_{\mu \nu}:=\lim _{n \rightarrow \infty} \frac{\mathcal{U}_{\mu v}}{n}=\frac{1}{(2 \pi)} \int_{-\pi}^{\pi} d \phi u_{\mu v}(\phi),
$$

where

$$
u_{\mu \nu}(\phi):=\frac{i}{4} \operatorname{Tr}\left\{\tilde{\gamma}(\phi)\left[\kappa_{\mu}(\phi), \kappa_{v}(\phi)\right]\right\}=\frac{i}{4} \operatorname{Tr}\left\{\kappa_{v}(\phi)\left[\tilde{\gamma}(\phi), \kappa_{\mu}(\phi)\right]\right\},
$$

In the above expression, $\kappa_{\mu}(\phi)$ is the symbol function of $K_{\mu}$, and it can be found as the operator solution of the $2 \times 2$ discrete Lyapunov equation

$$
\partial_{\mu} \tilde{\gamma}(\phi)=\tilde{\gamma}(\phi) \kappa_{\mu}(\phi) \tilde{\gamma}(\phi)-\kappa_{\mu}(\phi) .
$$

In the eigenbasis of $\tilde{\gamma}(\phi)$, with eigenvalues $\tilde{\gamma}_{j}$, the explicit solution of (279) reads

$$
\left(\kappa_{\mu}(\phi)\right)_{j k}=\frac{\left(\partial_{\mu} \tilde{\gamma}(\phi)\right)_{j k}}{1-\tilde{\gamma}_{j} \tilde{\gamma}_{k}} .
$$


Notice that the diagonal terms $\left(\kappa_{\mu}(\phi)\right)_{i j}$ provide vanishing contributions to Eq. (278), because they commute with $\tilde{\gamma}(\phi)$. Hence, Eq. (278) can be cast in the following basis independent form

$$
u_{\mu \nu}(\phi)=\left\{\begin{array}{ll}
\frac{i}{4} \frac{\operatorname{Tr}\left\{\tilde{\gamma}(\phi)\left[\partial_{\mu} \tilde{\gamma}(\phi), \partial_{\nu} \tilde{\gamma}(\phi)\right]\right\}}{(1-\operatorname{Det} \tilde{\gamma}(\phi))^{2}} & \operatorname{Det} \tilde{\gamma}(\phi) \neq 1 \\
0 & \operatorname{Det} \tilde{\gamma}(\phi)=1
\end{array} .\right.
$$

Notice that the condition Det $\tilde{\gamma}(\phi)=1$ is equivalent to having two eigenvalues of correlation matrix equal to $\left(\gamma_{i}, \gamma_{k}\right)=$ $\pm(1,1)$. This corresponds to the situation, already discussed in Section 16.1, in which two eigenmodes of the Gaussian state are pure. As already mentioned with regard to Eq. (252), such extremal values cause no singularity in MUC, but they rather result in a vanishing contribution to the MUC.

In the following, we will demonstrate that a singularity of $\overline{\mathcal{U}}$ signals the occurrence of a criticality. Specifically, employing the analytical extension in the complex plane of $u_{\mu \nu}(\phi)$ leads to

$$
\overline{\mathcal{U}}_{\mu \nu}=\sum_{\bar{z}^{\prime} \in S_{1}} \operatorname{Res}_{\bar{z}^{\prime}}\left[z^{-1} u_{\mu \nu}(z)\right] .
$$

Notice that $u_{\mu \nu}(z)$ has at most isolated poles, due to its rational dependence on $z$. Assume that, as $\lambda \rightarrow \lambda_{0} \in \mathcal{M}$, a pole $\bar{z}_{0}$ of $u_{\mu \nu}(z)$ approaches the unit circle from inside, which is the only condition under which $\overline{\mathcal{U}}$ is singular in $\lambda_{0}$. One can show that, whenever a pole $\bar{z}_{0}$ of $u_{\mu \nu}(z)$ approaches the unit circle, also a pole $\bar{z}$ of $\tilde{\gamma}(z)$ approaches the same value, causing the correlation length to diverge. Therefore the singular behaviour of the Uhlmann phase necessarily represents a sufficient criterion for a NESS-QPTs. Notice also that such criticalities are necessarily accompanied by the closure of the dissipative gap, however, the converse is in general not true. Indeed, a singularity in the MUC may only arise as the result of criticality and its existence does not depend on a vanishing dissipative gaps.

Let us now prove, that in translationally invariant models a vanishing dissipative gap is a necessary condition for criticality.

Proposition 2. If there exists a pole $\bar{z}_{0}(\lambda)$ of $\tilde{\gamma}(z)$, smoothly dependent of system parameters $\lambda \in \mathcal{M}$, such that $\lim _{\lambda \rightarrow \lambda_{0}}\left|\bar{z}_{0}\right|=$ 1 , then

$\Delta:=2 \min _{|z|=1, j} \operatorname{Re} x_{j}(z)=0$ for $\lambda=\lambda_{0}$.

Proof. Under the vectorising isomorphism, $A=a_{j k}|j\rangle\left\langle k\left|\rightarrow \operatorname{vec}(A):=a_{j k}\right| j\right\rangle \otimes|k\rangle$, the continuous Lyapunov Eq. (272) can be written as

$$
\hat{X}(z) \operatorname{vec}(\tilde{\gamma}(z))=\operatorname{vec}(y(z)),
$$

where $\hat{X}(z):=x(z) \otimes \mathbb{1}+\mathbb{1} \otimes x\left(z^{-1}\right)$. When $\operatorname{Det} \hat{X}(z) \neq 0$, the unique solution of the symbol function is found simply as

$$
\operatorname{vec}(\gamma(z))=\frac{\operatorname{vec}(\eta(z))}{d(z)}, \quad \text { where } \operatorname{vec}(\eta):=\operatorname{adj}(\hat{X}) \operatorname{vec}(y) .
$$

Here $\operatorname{adj}(\hat{X})$ stands for the adjugate matrix of $\hat{X}$ and $d(z):=\operatorname{Det} \hat{X}(z)$. The point in writing the solution in this form is that, by construction, $x(z)$ and $y(z)$ are polynomials in $z$ and $z^{-1}$ with coefficients smoothly dependent on the system parameters. Moreover, since determinant and adjugate matrix are always polynomial functions of a matrix coefficients, it results that also $\eta(z)$ and $d(z)$ will be two polynomials in $z$ and $z^{-1}$. Hence, $\tilde{\gamma}(z)$ 's poles are to be found among the roots $\bar{z}$ of $d(z)=0$. Thus, a necessary condition for criticality is that, for $\lambda \rightarrow \lambda_{0}$, a given root $\bar{z}$ approaches the unit circle $S_{1}$. This clearly means that for $\lambda=\lambda_{0}$ there must exist $\bar{z}_{0}$ such that $\left|\bar{z}_{0}\right|=1$ and $d(z)=\operatorname{Det} \hat{X}\left(\bar{z}_{0}\right)=0$, which implies a vanishing dissipative gap $\Delta:=2 \min _{|z|=1, j} \boldsymbol{\operatorname { R e }} x_{j}(z)$, where $x_{j}(z)$ 's are the eigenvalues of $\tilde{x}(z)$ [390].

On the other hand, the converse of the above proposition is not true: a vanishing dissipative gap is not a sufficient condition for criticality, but only necessary. Indeed, it may well be the case that all those roots $\bar{z}$ which approach the unit circle as $\lambda \rightarrow \lambda_{0}$ are removable singularities of (284). This would result in a finite correlation length, even when $\Delta \rightarrow 0$. The fact that this actually happens can be readily checked with the example in Section 18.1.

We will next show that a singular behaviour of $\mathcal{U}$ with respect to the parameters is a sufficient condition for criticality. First of all, notice, from Eq. (281), that $u(\phi)$ may depend on the dynamics only through $\tilde{\gamma}$, hence any closure of the gap which does not affect the analytical properties of $\tilde{\gamma}$ cannot result in a singular behaviour of $\mathcal{U}$ (see also Lemma 2 in the following). We will just need to show that a necessary condition for a singular behaviour of $u(\phi)$ is $\Delta=0$.

Indeed, let us now show that the poles of $u_{\mu \nu}(z)$ with $|z|=1$ are to be found only among the roots of $d(z)$. Assuming $d(z) \neq 0$, and plugging the unique solution (284) into Eq. (281) leads to

$$
u_{\mu \nu}(z)=\frac{N(z)}{D(z)}=\frac{i}{4} \frac{d(z) \operatorname{Tr}\left\{\eta(z)\left[\partial_{\mu} \eta(z), \partial_{\nu} \eta(z)\right]\right\}}{\left(d(z)^{2}-\operatorname{Det} \eta(z)\right)^{2}},
$$

where the numerator $N(z)$ and denominator $D(z)$ are polynomials in $z$ and $z^{-1}$ with smooth dependence on $\lambda$ 's.

We will demonstrate the following: (i) that all roots of $d(z)$ such that $|z|=1$ are also roots of $D(z)$, and (ii) that any other roots of $D(z)$, such that $|z|=1$, are not poles of $u_{\mu v}(z)$. For the statement $(i)$, it is just enough to prove the following lemma. 
Lemma 2. If $d(z)=0$ with $|z|=1$, then $\eta(z)=0$.

Proof. For $|z|=1$, let us write explicitly $z=e^{i \phi}$. It is not hard to show that from its definition, the matrix $\tilde{x}(\phi)$ enjoys the following property $\tilde{x}(\phi)^{\dagger}=\tilde{x}(-\phi)^{T}$. Correspondingly, the eigenvalues of $\hat{X}$ are $x_{j}+x_{k}^{*}$ with $j, k=1,2$, where $x_{j}$ are the eigenvalues of $\tilde{x}(\phi)$. Since $\operatorname{Re} x_{j} \geq 0$, Det $\hat{X}=0$ implies that there must exist an eigenvalue $x_{0}$ of $\tilde{x}(\phi)$ with vanishing real part, hence $\Delta=2 \min _{j} \operatorname{Re} x_{j}=2 \operatorname{Re} x_{0}=0$. If $|0\rangle$ is the eigenstate of $\tilde{x}(\phi)$ with eigenvalue $x_{0}$, then

$$
x_{0}+x_{0}^{*}=\left\langle 0\left|\tilde{x}(\phi)+\tilde{x}(-\phi)^{T}\right| 0\right\rangle=4\left\langle 0\left|\tilde{m}(\phi)+\tilde{m}(-\phi)^{T}\right| 0\right\rangle=0,
$$

where in the second equality we used the definition $\tilde{x}(\phi):=2\left[2 i \tilde{h}(\phi)+\tilde{m}(\phi)+\tilde{m}^{T}(-\phi)\right]$ and the antisymmetry $\tilde{h}(\phi)=-\tilde{h}(-\phi)^{T}$. From the non-negativity of the $\tilde{m}(\phi)$ matrices, it follows that $\langle 0|\tilde{y}(\phi)| 0\rangle=-4\left\langle 0\left|\tilde{m}(\phi)-\tilde{m}(-\phi)^{T}\right| 0\right\rangle=0$.

In Lemma 3 of Appendix B it is shown that when $2 \mathbf{R e} x_{0}=0$, the geometric multiplicity of $x_{0}$ is equal to its algebraic multiplicity, hence the $2 \times 2$ matrix $\tilde{x}(\phi)$ is diagonalisable. Then, let $|j\rangle$ be the set of eigenstates with eigenvalues $x_{j}$. In the eigenbasis $|j\rangle \otimes|k\rangle, j, k=0,1$ the adjugate matrix has the following diagonal form,

$$
\operatorname{adj}(\hat{X})=2\left(\begin{array}{cccc}
\left|x_{0}+x_{1}^{*}\right|^{2} \mathbf{R e}\left(x_{1}\right) & 0 & 0 & 0 \\
0 & 2\left(x_{0}+x_{1}^{*}\right) \mathbf{R e}\left(x_{1} x_{0}\right) & 0 & 0 \\
0 & 0 & 2\left(x_{1}+x_{0}^{*}\right) \mathbf{R e}\left(x_{1} x_{0}\right) & 0 \\
0 & 0 & 0 & \left|x_{1}+x_{0}^{*}\right|^{2} \mathbf{R e}\left(x_{0}\right)
\end{array}\right)
$$

and due to $\operatorname{Re} x_{0}=0$ all elements, but $\langle 0,0|\operatorname{adj}(\hat{X})| 0,0\rangle$, vanish. On the other hand, the element $\operatorname{vec}(\tilde{y})_{00}:=\langle 0|\tilde{y}| 0\rangle=0$, $\operatorname{implying} \operatorname{vec}(\eta)=\operatorname{adj}(\hat{X}) \operatorname{vec}(y)=0$.

To prove statement (ii), we just need the following proposition.

Proposition 3. If $\bar{z}_{0}$ is a root of $D(z)$ with $\left|\bar{z}_{0}\right|=1$, and $d\left(\bar{z}_{0}\right) \neq 0$, then $u_{\mu \nu}(z)$ is analytic in $z_{0}$.

Proof. Let $\bar{z}_{0}$ be a root of $D(z)$ with $\left|\bar{z}_{0}\right|=1$, with the assumption that $d\left(\bar{z}_{0}\right) \neq 0$. Notice that whenever $d(z) \neq 0, \tilde{\gamma}(z)$ in (284) is the unique solution of the Lyapunov Eq. (272). As such, it is analytic in $z$ (and smoothly dependent on $\lambda$ 's). Since

$$
D(z):=\left(d(z)^{2}-\operatorname{Det} \eta(z)\right)^{2}=d(z)^{4}[1-\operatorname{Det} \tilde{\gamma}(z)]^{2},
$$

we obviously have Det $\tilde{\gamma}\left(\bar{z}_{0}\right)=1$. Just observe that if $\gamma(z)$ is an analytic, smoothly dependent on the system parameters $\lambda \in \mathcal{M}, u_{\mu \nu}(z)$ may be singular in $\bar{z}_{0}$ only if $\operatorname{Det} \tilde{\gamma}\left(\bar{z}_{0}\right)=1$. Assume then $\operatorname{Det} \tilde{\gamma}\left(\bar{z}_{0}\right)=1$, then either $\gamma\left(\bar{z}_{0}\right)= \pm \mathbb{1}$. Without loss of generality, we can write $\tilde{\gamma}(z)=\mathbb{1}+T\left(z-\bar{z}_{0}\right)^{2 n}+\mathcal{O}\left(z-\bar{z}_{0}\right)^{2 n}, n \in \mathbb{N}$, where $T=T^{\dagger}$ is the first non-vanishing term of the Taylor expansion of $\tilde{\gamma}(z)-\mathbb{1}$. The fact that this term must be of even order $(2 n)$ is due to the positive semidefiniteness of the $\mathbb{1}-\tilde{\gamma}(z)$ for $z \in S_{1}$. By expressing the $2 \times 2$ matrix $T$ in terms of Pauli matrices, $T=t_{0} \mathbb{1}+\boldsymbol{t} \cdot \boldsymbol{\sigma}$, where $\boldsymbol{\sigma}:=\left(\sigma_{x}, \sigma_{y}, \sigma_{z}\right)^{T}, t_{0} \in \mathbb{R}$ and $\boldsymbol{t} \in \mathbb{R}^{3}$, the positive semi-definiteness condition above reads: $t_{0}<0$ and $\|\boldsymbol{t}\| \leq\left|t_{0}\right|$. Plugging the Taylor expansion in (281) and retaining only the first non-vanishing terms, yields

$$
u_{\mu \nu}(z)=-\frac{1}{4} \frac{\boldsymbol{t} \cdot\left(\partial_{\mu} \boldsymbol{t} \wedge \partial_{\nu} \boldsymbol{t}\right)}{t_{0}^{2}}\left(z-\bar{z}_{0}\right)^{2 n}+o\left(z-\bar{z}_{0}\right)^{2 n} .
$$

We have thus proven that a non-analyticity of $u_{\mu \nu}(z)$ in $\bar{z}_{0} \in S_{1}$ is necessarily due to a pole $\bar{z}$ of $\tilde{\gamma}(z)$ approaching $\bar{z}_{0}$, as $\lambda \rightarrow \lambda_{0}$, resulting in a diverging correlation length. Therefore, a singular behaviour of $\overline{\mathcal{U}}$ in the manifold $\mathcal{M}$ is a sufficient criterion for criticality.

\section{Applications}

\subsection{Vanishing dissipative gap without criticality}

The primary scope of this subsection is not discussing a model which may be relevant per se, rather it serves to illustrate in a simple translationally invariant case the ambiguous relation between criticality and vanishing dissipative gap. As a byproduct, one may also appreciate the sensitivity of the Uhlmann curvature to the criticality and its unresponsiveness to the gap. Specifically, in this section we will describe an example of a 1D Fermionic system in which the closure of the dissipative gap does not necessarily lead to a diverging correlation length. Consider a chain of Fermions on a ring geometry, driven uniquely by an engineered reservoir, i.e. with no Hamiltonian. The reservoir is described by the following set of jump operators

$$
\Lambda(r)=\left[(1+\lambda) \boldsymbol{l}_{0}^{T} \boldsymbol{\omega}_{r}+\boldsymbol{l}_{1}^{T} \boldsymbol{\omega}_{r+1}+\lambda \boldsymbol{l}_{2}^{T} \boldsymbol{\omega}_{r+2}\right] / n(\lambda),
$$

where $r=1, \ldots, n, \boldsymbol{l}_{0}=(\cos \theta,-\sin \theta)^{T}, \boldsymbol{l}_{1}=\boldsymbol{l}_{2}=i(\sin \theta, \cos \theta)^{T}$, and $n(\lambda)=4\left(\lambda^{2}+\lambda+1\right)$, with $\lambda \in \mathbb{R}$ $\theta=[0,2 \pi)$. This is a simple extension of a model introduced in [382], which, under open boundary conditions, shows a dissipative topological phase transitions for $\lambda= \pm 1$. In the thermodynamic limit $n \rightarrow \infty$, the eigenvalues of $\tilde{x}(\phi)$ are 

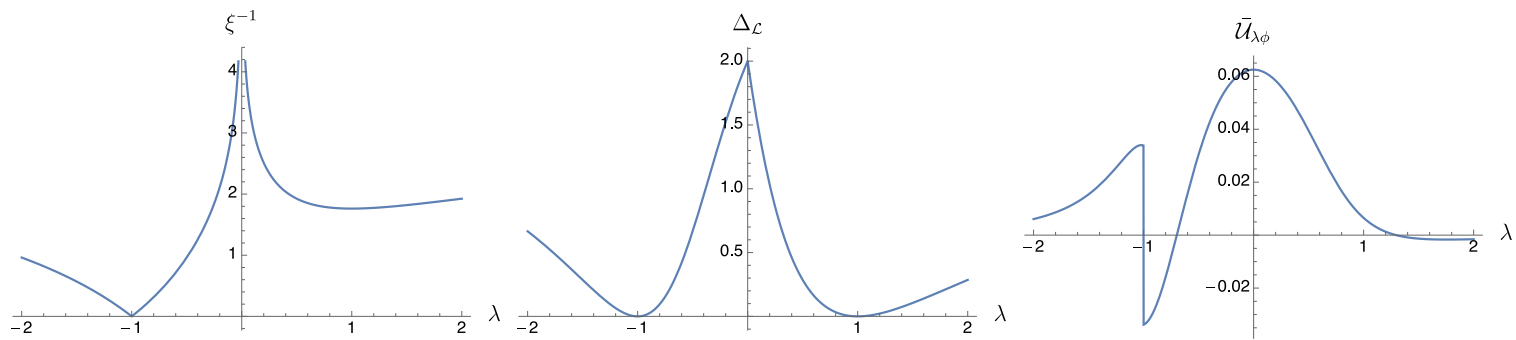

Fig. 8. Model of a 1D Fermionic chain on a ring showing a closing dissipative gap that does not imply a diverging correlation length. This is the model discussed in Section 18.1 which is an extension of a model introduced in [382]. The inverse correlation length, the dissipative gap and the MUC are shown, respectively, from the left to the right panel. The model is critical only for $\lambda=-1$, while the gap closes for both $\lambda= \pm 1$. As expected, the discontinuity of MUC captures the criticality, and it is otherwise insensitive to a vanishing dissipative gap.

$x_{1}=4(1+\lambda)^{2} / n(\lambda)^{2}$, and $x_{2}=4\left(1+2 \lambda \cos \phi+\lambda^{2}\right) / n(\lambda)^{2}$, showing a closure of the dissipative gap at $\lambda= \pm 1$. For $|\lambda| \neq 1$ the unique NESS is found by solving the continuous Lyapunov Eq. (272). The symbol function, in a Pauli matrix representation, results $\tilde{\gamma}(\phi)=\boldsymbol{\gamma}^{T} \cdot \boldsymbol{\sigma}$, where $\boldsymbol{\sigma}:=\left(\sigma_{x}, \sigma_{y}, \sigma_{z}\right)^{T}$, and

$$
\boldsymbol{\gamma}=g(\phi)\left[\begin{array}{c}
(\sin \phi+\lambda \sin 2 \phi) \cos 2 \theta \\
(\cos \phi+\lambda \cos 2 \phi) \\
-(\sin \phi+\lambda \sin 2 \phi) \sin 2 \theta
\end{array}\right],
$$

where $g(\phi)=(1+\lambda) /\left(1+\lambda+\lambda \cos \phi+\lambda^{2}\right)$, with eigenvalues $\pm g(\phi) \sqrt{1+\lambda^{2}+2 \lambda \cos \phi}$. This shows that $\tilde{\gamma}$ is critical in the sense of diverging correlation, only for $\lambda=-1$ and not for $\lambda=1$, even if the dissipative gap closes in both cases. Fig. 8 shows the dependence of the inverse correlation length of the bulk, the dissipative gap and the mean Uhlmann curvature $\overline{\mathcal{U}}_{\lambda \phi}$ on the parameter $\lambda$. Notice a discontinuity of the Uhlmann phase corresponding to the critical point $\lambda_{0}=-1$, while it does not show any singularity for $\lambda=1$ where the gap closes.

\subsection{Rotated $X Y$-model with local dissipation}

Let us now turn to a prototypical example of a translationally invariant one-dimensional model. The features described above are exemplified in the rotated XY model with periodic boundary conditions [31,37], $H=R(\theta) H_{X Y} R(\theta)^{\dagger}$, with $R(\theta)=e^{-i \frac{\theta}{2} \sum_{j} \sigma_{j}^{z}}$ and

$$
H_{X Y}=\sum_{j=1}^{n}\left(\frac{1+\delta}{2} \sigma_{j}^{x} \sigma_{j+1}^{x}+\frac{1-\delta}{2} \sigma_{j}^{y} \sigma_{j+1}^{y}+h \sigma_{j}^{z}\right),
$$

where each site $j$ is coupled to two local reservoirs with Lindblad operators $\Lambda_{j}^{ \pm}=\epsilon \mu_{ \pm} \sigma_{j}^{ \pm}$. The spin-system is converted into a quadratic Fermionic model via Jordan-Wigner transformations. The Liouvillian spectrum can be solved exactly $[123,381,390]$ and it is independent of $\theta$. In the weak coupling limit $\epsilon \rightarrow 0$, the symbol function of the NESS correlation matrix reads $\tilde{\gamma}(\phi)=\boldsymbol{\gamma}^{T} \cdot \boldsymbol{\sigma}$, where

$$
\boldsymbol{\gamma}=g(\phi)\left(\begin{array}{c}
t(\phi) \cos \theta \\
-1 \\
t(\phi) \sin \theta
\end{array}\right)
$$

with $g(\phi)=\frac{\mu_{-}^{2}-\mu_{+}^{2}}{\mu_{-}^{2}+\mu_{+}^{2}} \frac{1}{1+t(\phi)^{2}}$ and $t(\phi):=\delta \sin \phi /(\cos \phi-h)$. The system shows criticality in the same regions as the $X Y$ Hamiltonian model [381]. By using expression (282) one can calculate the exact values of the mean Uhlmann curvature. One finds that $\overline{\mathcal{U}}_{\delta h}$ vanishes identically, while $\overline{\mathcal{U}}_{\delta \theta}$ and $\overline{\mathcal{U}}_{h \theta}$ are plotted in Fig. 9. As predicted, the Uhlmann curvature shows a singular behaviour only across criticality. In particular, $\mathcal{U}_{h \theta}$ is discontinuous in the $X Y$ critical points $|h|=1$, while $\mathcal{U}_{\delta \theta}$ is discontinuous in the $X X$ type criticalities $\delta=0, h<1$. This shows that the mean Uhlmann curvature captures faithfully the critical behaviour of the underlying physical model. In the following we will see a model with a richer phase diagram, in which the geometric features of criticality will be even more apparent.

\subsection{Boundary driven $X Y$-model}

Let us apply the above analysis to a specific model, the boundary-driven spin-1/2 XY chain [123]. In this model, an open chain of spin-1/2 particles interacts via the $X Y$-Hamiltonian,

$$
H_{X Y}=\sum_{j=1}^{n-1}\left(\frac{1+\delta}{2} \sigma_{j}^{x} \sigma_{j+1}^{x}+\frac{1-\delta}{2} \sigma_{j}^{y} \sigma_{j+1}^{y}\right)+\sum_{j=1}^{n} h \sigma_{j}^{z},
$$



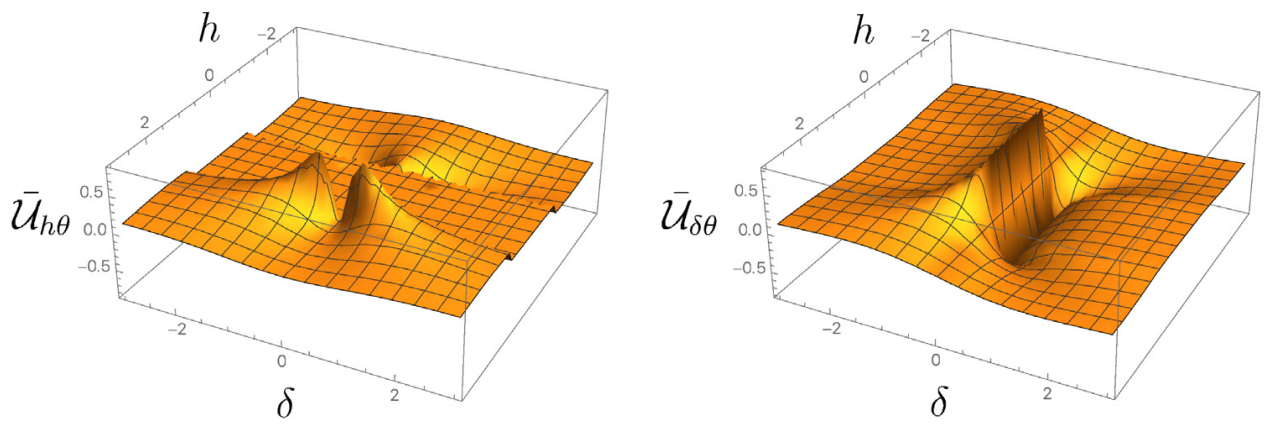

Fig. 9. The mean Uhlmann curvature per number of sites $\overline{\mathcal{U}}$ for the rotated XY model with local reservoirs. The dependence of $\overline{\mathcal{U}}_{h \theta}$ (left) and of $\overline{\mathcal{U}}_{\delta \theta}$ (right) on the parameters $\delta$ e $h$. The mean Uhlmann curvature shows a singular behaviour in the critical regions of the model. $\mathcal{U}_{h \theta}$ is discontinuous in the $X Y$ critical points $|h|=1$, and $\mathcal{U}_{\delta \theta}$ is discontinuous in the $X X$ type criticalities $\delta=0, h<1$.

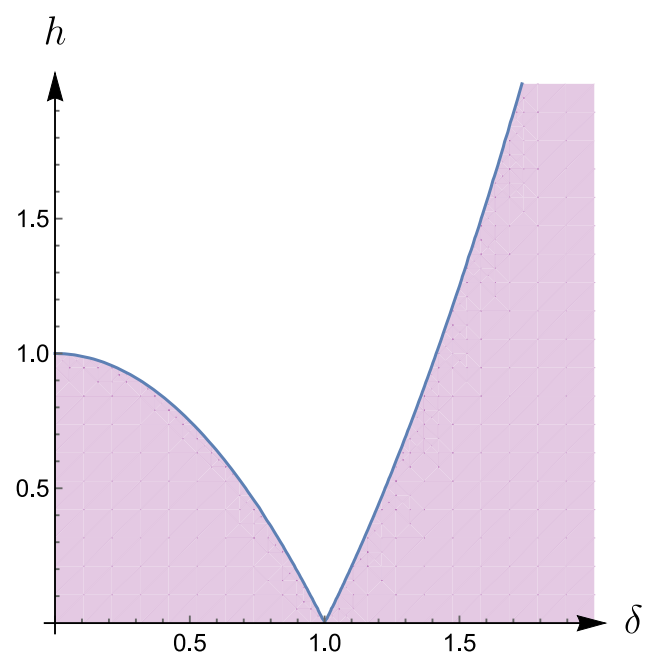

Fig. 10. Phase diagram of the boundary driven XY model. For $h<h_{c}:=\left|1-\delta^{2}\right|$ the chain exhibits long-range magnetic correlations (LRMC). For $h>h_{c}$ and along the lines $h=0$ and $\delta=0$ the model shows short-range magnetic correlations (SRMC). The qualitative features of the phase diagram do not depend on the values of the environmental parameters $\kappa_{L}^{ \pm}$and $\kappa_{R}^{ \pm}$.

where the $\sigma_{j}^{x, y, z}$ are Pauli operators acting on the spin of the $j$ th site. At each boundary, the chain is in contact with two different reservoirs, described by Lindblad operators

$$
\Lambda_{L}^{ \pm}=\sqrt{\kappa_{L}^{ \pm}}\left(\sigma_{j}^{x} \pm i \sigma_{j}^{y}\right) / 2 \quad \text { and } \quad \Lambda_{R}^{ \pm}=\sqrt{\kappa_{R}^{ \pm}}\left(\sigma_{j}^{x} \pm i \sigma_{j}^{y}\right) / 2 .
$$

A Jordan-Wigner transform converts the system into a quadratic Fermionic dissipative model with Gaussian NESS [123,364]. The system experiences different phases as the anisotropy $\delta$ and magnetic field $h$ are varied. For $h<$ $h_{c}:=\left|1-\delta^{2}\right|$ the chain exhibits long-range magnetic correlations (LRMC) and high sensitivity to external parameter variations (see Fig. 10). For $h>h_{c}$ and along the lines $h=0$ and $\delta=0$ the model shows short-range magnetic correlations (SRMC), with correlation function $C_{j k}:=\left\langle\sigma_{j}^{z} \sigma_{k}^{z}\right\rangle-\left\langle\sigma_{j}^{z}\right\rangle\left\langle\sigma_{k}^{z}\right\rangle$ exponentially decaying: $C_{j k} \propto \exp -|j-k| / \xi$, with $\xi^{-1} \simeq 4 \sqrt{2\left(h-h_{c}\right) / h_{c}}$. In both long and short range phases, the dissipative gap closes as $\Delta=\mathcal{O}\left(n^{-3}\right)$ in the thermodynamic limit $n \rightarrow \infty$. The critical line $h=h_{c}$, is characterised by power-law decaying correlations $C_{j k} \propto|j-k|^{-4}$, and $\Delta=\mathcal{O}\left(n^{-5}\right)$. Therefore, the scaling law of $\Delta$ cannot distinguish long and short range phases, and can only detect the actual critical line $h=h_{c}$. Likewise, $\Delta$ does not identify the transitions from the LRMC phase to the $\delta=0$ and $h=0$ lines.

In Table 1 , the scaling laws of $|\mathcal{U}|,\|g\|_{\infty}$, $\operatorname{Det}(g)$ and $\Delta$ are compared in each region of the phase diagram. Figs. 11 and 12 clearly show that both $\|g\|_{\infty}$, and $\left|\mathcal{U}_{\delta h}\right|$ map faithfully the phase diagram of Fig. 10 . The results of Table 1 show that the Liouvillian gap, the metric and the MUC encode different information. Indeed, unlike the Hamiltonian gap ruling the ground-state QPT, the Liouvillian gap $\Delta$ closes for $n \rightarrow \infty$ at the critical point and for $h \neq h_{c}$, both in the LRMC and SRMC phase. As the reservoirs act only at the boundaries of the spin chain, the eigenvalues $x_{k}$ of the matrix $X$ for $n \gg 1$ 
Table 1

Here we show a comparison between the scaling laws for: the dissipative gap $\Delta$ [123], the largest eigenvalue $\|g\|_{\infty}$ of the metric [177], the determinant of $g$, the largest eigenvalue $\|\mathcal{U}\|_{\infty}=\left|\mathcal{U}_{\delta h}\right|=\sqrt{\operatorname{Det} \mathcal{U}}$ of the MUC, and the ratio $R:=|\operatorname{Det} 2 \mathcal{U}| / \operatorname{Det} J \propto \operatorname{Det} 2 \mathcal{U} \mid / \operatorname{Det}$ for each phase of the boundary driven XY model [123]. The ratio $R \leq 1$ when $R \sim n^{0}$ marks the condition of maximal asymptotic incompatibility [111] introduced in Section 10.

\begin{tabular}{lllllll}
\hline Phase & Parameters & $\Delta$ & $\|g\|_{\infty}$ & Det $g$ & $\left|\mathcal{U}_{\delta h}\right|$ & $\mathrm{R}$ \\
\hline Critical & $h=0$ & $n^{-3}$ & $n^{6}$ & $n^{7}$ & $n^{3}$ & $n^{-1}$ \\
Long range & $0<|h|<h_{c}$ & $n^{-3}$ & $n^{3}$ & $n^{4}$ & $n^{2}$ & $n^{0}$ \\
Critical & $h \simeq h_{c}$ & $n^{-5}$ & $n^{6}$ & $n^{7}$ & $n^{0}$ & $n^{-7}$ \\
Short range & $h>h_{c}$ & $n^{-3}$ & $n$ & $n^{2}$ & $n^{0}$ & $n^{-2}$ \\
Critical & $\delta=0,|h|<h_{c}$ & $n^{-3}$ & $n^{2}$ & $n^{8}$ & $n^{3}$ & $n^{-2}$ \\
\hline
\end{tabular}

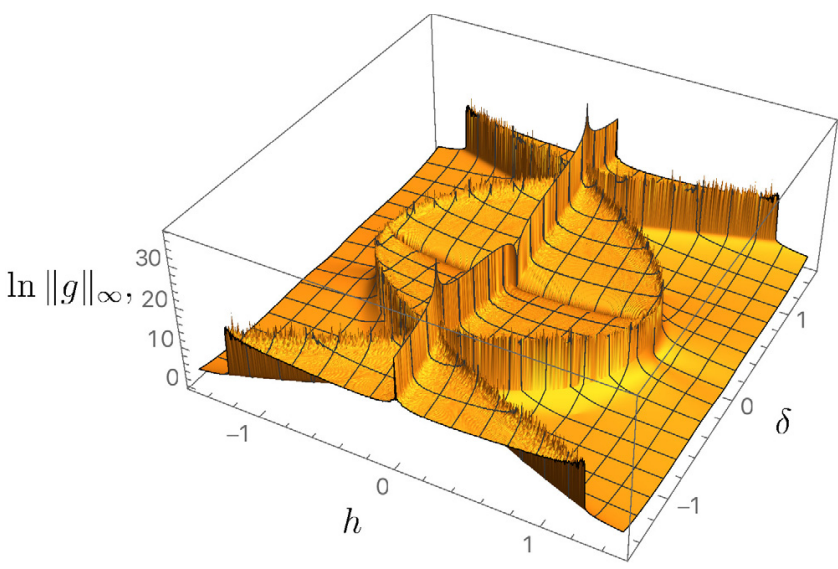

Fig. 11. The largest eigenvalue of the Bures metric $\|g\|_{\infty}$ for the boundary driven $X Y$ model, for $n=300$. The qualitative behaviour of the metric maps the phase diagram quite faithfully. It is evident a larger value of $\|g\|_{\infty}$ close to the phase transitions $h=h_{c}:=\left|1-\delta^{2}\right|$ between LRMC and short range phases. $\kappa_{L}^{+}=0.3, \kappa_{L}^{-}=0.5, \kappa_{R}^{+}=0.1, \kappa_{R}^{-}=0.5$. The qualitative features remain unchanged for different values of $\kappa_{L, R}^{ \pm}$(results not shown).

are a small perturbation of the $n \rightarrow \infty$ case, where $x_{k}= \pm 4 i \varepsilon_{k}$, being

$$
\varepsilon_{k}=\sqrt{(\cos k-h)^{2}+\gamma^{2} \sin ^{2} k}
$$

the quasi-particle energy dispersion relation of the Hamiltonian (289). In particular $x_{k}$ gains a small real part and one finds a gap $\Delta=O\left(n^{-3}\right)$ for $h \neq h_{c}$ and $\Delta=O\left(n^{-5}\right)$ for $h=h_{c}$. Therefore the scaling of the Liouvillian gap allows one to identify the transitions from the SRMC phase to the LRMC phase only along the critical line $h=h_{c}$, while the transitions occurring at the $h=0$ (or $\gamma=0$ ) line can only be appreciated by evaluating the long-rangeness of the magnetic correlations. The question that naturally arises is how the different phases and transitions can be precisely characterised in a way similar to what happens for ground state quantum phase transitions. This question becomes more compelling if one compares the above results with the scaling of the Bures metric $g_{\mu \nu}$ and mean Uhlmann curvature $\mathcal{U}$ (more precisely their largest eigenvalue $\|g\|_{\infty}$ and $\|\mathcal{U}\|_{\infty}=\left|U_{\delta h}\right|^{6}$ ) for specific values of the parameters, see Table 1, Figs. 11 and 12.

A first important result is that the geometric properties $g$ and $\mathcal{U}$ are able to identify the transitions between SRMC and LRMC phases. On the "transitions lines" $h=0$ and $h=h_{c}$ one has that $\|g\|_{\infty} \sim \mathcal{O}\left(n^{6}\right)$, while in the rest of the phase diagram $\|g\|_{\text {infty }}<\mathcal{O}\left(n^{6}\right)$. Furthermore, a closer inspection of the elements of $g$ shows that, while $g_{h h}(h=0, \gamma)=\mathcal{O}\left(n^{6}\right)$, one has that $g_{\gamma \gamma}(h=0, \gamma)=\mathcal{O}(n)$ : the scaling is superextensive only if one moves away from the line $h=0\left(g_{h h}\right)$ and enters in the LRMC phase, while, if one moves along the $h=0$ line $\left(g_{\gamma \gamma}\right)$, i.e. if one remains in the SRMC phase, the scaling is simply extensive and it matches the scaling displayed in the other SRMC phase $h>h_{c}$. On the other hand, the transitions occurring at $\gamma=0$ has a different scaling: $g_{\gamma \gamma}=\mathcal{O}\left(n^{2}\right)$ while $g_{h h} \approx 0$.

Another important result shown in Table 1 is that both the metric tensor and the MUC are able to signal the presence of long-range correlations: within the LRMC phase $g_{\mu \nu}$ scales superextensively with $\|g\|_{\infty} \sim \mathcal{O}\left(n^{3}\right)$, and $\left|\mathcal{U}_{h \delta}\right| \sim \mathcal{O}\left(n^{2}\right)$. This super-extensive behaviour is different from that displayed at the transitions lines. Thus, differently from $\Delta$, the MUC discriminates these phases, with no need of crossing the critical line $h=h_{c}$.

However, a more compelling result concerns the quality of the phase transitions in each region. As discussed in Section 10.2, the MUC marks the role played by the quantum nature of the model in the parameter estimation problem. In

$6 \mathcal{U}$ is an antisymmetric $2 \times 2$ matrix for this two-parameter model. Therefore, it only has two opposite eigenvalues $\pm\left|U_{\delta h}\right|$. 


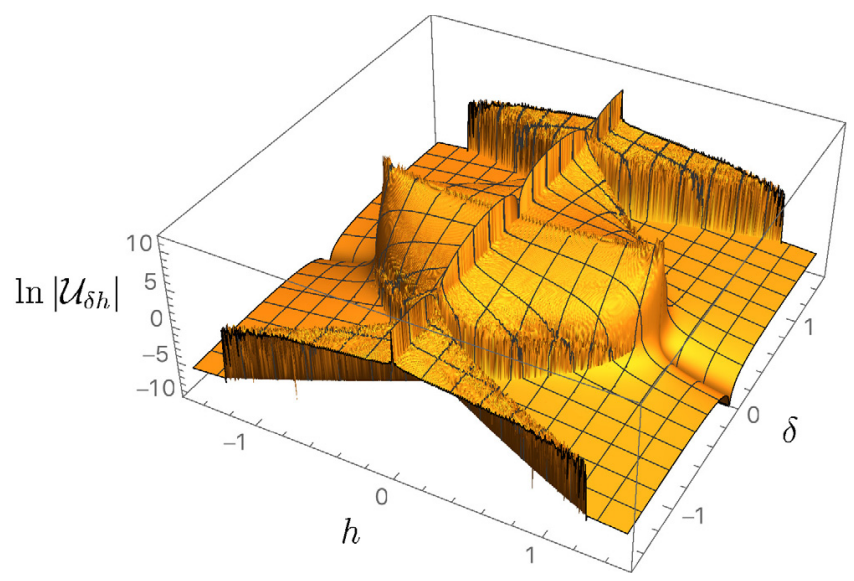

Fig. 12. The MUC $\left|\mathcal{U}_{\delta h}\right|$ for the boundary driven XY model, for $n=300$. Here the parameters are the same as in Fig. 11 . As in the case of the metric, also the qualitative behaviour of MUC maps quite well the phase diagram. The striking difference with Fig. 11 is the nature of the discontinuity across the critical line $h=h_{c}:=\left|1-\delta^{2}\right|$, which still signals the transitions between LRMC and short range phases. Here the lack of a greater divergence of the MUC at the critical line is a manifestation of the classical nature in the fluctuations driving the NESS-QPTs.

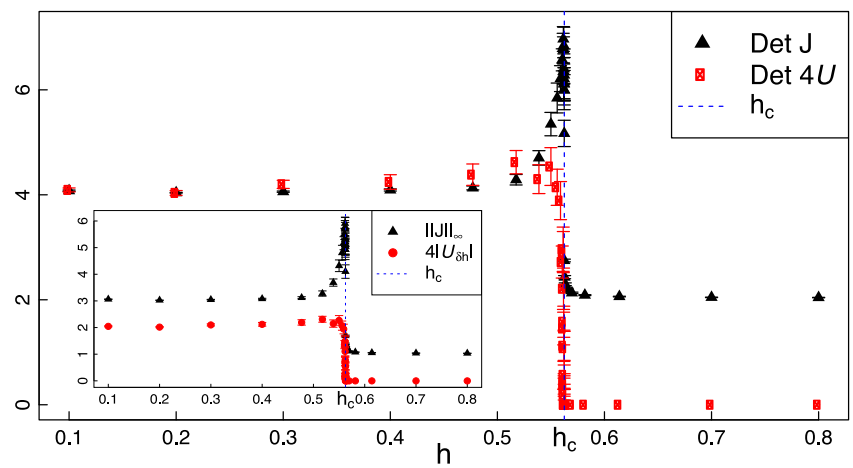

Fig. 13. Boundary driven XY model. Scaling laws of the determinants (main) and maximal eigenvalues (inset) of the Fisher information matrix $J$ and mean Uhlmann curvature $\mathcal{U}$ for different values of $h$, with $\delta=1.25$ and $h_{c}=\left|1-\delta^{2}\right|$. The laws do not depend on the particular values of the $\kappa_{R, L}^{ \pm}$. The scalings are the results of fits on numerical data, with size ranging in $n \in[20,2000]$.

other words, it signals the "quantumness" of the underlying physical model. Table 1 displays the scaling law of the ratio $R=\sqrt{\mid \text { Det2U|/Det } J}$ in different regions of the phase diagram. In particular, its asymptotic behaviour provides insight into the character of fluctuations which drive the NESS-QPTs. Indeed, in the limit of $R \sim$ const this ratio signals a condition of maximal asymptotic incompatibility, in which the role of the quantum fluctuations in the criticality cannot be neglected in the thermodynamic limit.

Fig. 13 shows that, in the LRMC phase, the scaling law of the MUC saturates the upper bound (158), in contrast to the short range phase. This shows the striking different nature of the two phases. In the LRMC region, the system behaves as an inherently two-parameter quantum estimation model, where the parameter incompatibility cannot be neglected even in the thermodynamic limit. On the short-range phase, instead, the system is asymptotically quasi-classical. The critical line $\delta=0$ (with $|h| \leq h_{c}$ ) and the critical line $h=0$, which mark regions of short range correlations embedded in a LRMC phase, show a MUC which grows super-extensively, with scaling $\mathcal{O}\left(n^{3}\right)$, and a nearly saturated inequality (158). In the critical line $h \simeq h_{c}$, despite the spectacular divergence of $\|g\|_{\infty} \simeq \mathcal{O}\left(n^{6}\right)$, the scaling law of $\left|\mathcal{U}_{\delta h}\right|$ drops to a constant, revealing an asymptotic quasi-classical behaviour of the model at the phase transitions.

\section{Conclusions}

Condensed matter physics is a rich framework where a variety of interesting phenomena arise in association with geometrical properties of the interactions. Topological behaviour of quantum interactions are particularly evident for those systems near QPTs [7,400]. It is a well known fact that quantum criticalities are accompanied by a qualitative change in the nature of correlations in the ground state of a quantum system, and describing these changes is clearly one of the major interests in condensed matter physics. Typical examples are metal-insulator transitions, or paramagnetic-ferromagnetic 
transitions for spin chains, where the two phases are associated with distinct local vs. global properties of the quantum state.

It is, therefore, expected that such drastic changes in the properties of the ground states are reflected in the geometry of the Hilbert space explored by the system across the criticality. Geometric phase is known to be a signature of the curvature, and in general of the geometry of the state manifold, and therefore it is a useful means to investigate the properties of systems near QPTs. The heuristic explanation for the non-trivial behaviour of Berry curvature in the proximity of criticality relies on the idea of level crossings, occurring at the thermodynamic limit, which involve ground state and low lying part of the energy spectrum. Level crossings can be identified as the origin of the curvature in the phase space manifold, pretty much the same way energy singularity bends the geometry of the space-time, or more like a Dirac monopole twists the topology of the field configuration around it [26]. Driving the system close to or around these singularities results in dramatic effects on the state geometry, picked up by its kinematics in the form of geometric phase instabilities [31,32]. Whether this intuition may be adapted to an entirely different type of quantum many-body phenomena is an absolutely non-trivial question.

Novel type of non-equilibrium phase transitions has recently emerged in quantum mechanical systems, as phenomena that may underpin new forms of criticality, departing significantly from transitions that are observed in the equilibrium settings. A particularly intriguing type of non-equilibrium critical phenomena arises in the context of open quantum systems, where the non-equilibrium character is induced by coupling between system and several external reservoirs. Theoretical investigation of open quantum systems is ultimately motivated by the inherently open nature of several current experimental platforms, which are typically subject to external drive, dissipation and dephasing.

The occurrence of quantum phase transitions in non-equilibrium steady states, which are the results of complex manybody dissipative evolutions, is far from being understood. Harnessing the investigation of such an uncharted scenario with a powerful new set of tools is certainly desirable. Geometric properties have proven successful in unravelling general quantitative and qualitative informations in equilibrium criticalities. It is therefore expected that they may glean new insights is these novel scenario, providing a comprehensive framework for their understanding.

The crucial focus of this review was indeed to present the general framework of geometric methods and to demonstrate the applicability of these ideas to the entirely novel category of non-equilibrium phase transitions. What it may seem at first glance a pedantic application of known concepts to yet another instance of critical phenomena, is instead quite a major shift of paradigm. It is no coincidence that nearly 10 years after their introduction, models such as the one proposed in Ref. [123] are still subject of active investigations through well established tools of quantum information and information geometry. Yet, a full understanding of their main characteristics is still lacking. Most of all, what is missing is an intuitive comprehensive framework, within which comparing the non-equilibrium-QPTs with what is known from equilibrium phase transitions.

Equilibrium phase transitions fall invariably into two markedly non-overlapping categories: classical phase transitions and quantum phase transitions. NESS-QPTs offer a unique arena where such a distinction indeed fades off. The coexistence between quantum and classical fluctuations in these models may vaguely be reminiscent of what happens at quantum to classical crossovers in equilibrium phenomena, with a major striking difference: the remarkably sharp character of truly critical phenomena.

Among other things, we described a recently developed approach which allows one to quantitatively assess the "quantumness" of a critical phenomena. This method resorts to ideas borrowed from quantum estimation theory, which endow the geometric phase approach with an operationally well defined character. This approach brings insight into the interplay between quantum and classical fluctuation in critical phenomena. Quadratic Fermionic Liouvillian models are perhaps the simplest significant example where this interaction plays a non-trivial role, where one finds quite unusual interplay between markedly classical and quantum features associated to the same phase transitions.

A source of confusion within the class of dissipative NESS-QPTs is that the concept of criticality has indeed a variety of inequivalent definitions. Already, in the physically relevant subclass of quadratic quasi-free Fermionic models, there are two non-equivalent widely used definitions in literature. They rely on the idea of diverging correlation length and critical slow down, respectively. While in the usual setting of equilibrium QPTs these two definitions generally coincide, in NESSQPTs this is not quite the case. The first definition introduced by Eisert and Prosen in [191] is the one mostly adopted in this review. The second, inequivalent, one is used for example in a series of works related to dissipatively induced topological order (e.g. [382]). One can prove analytically that a singular geometric phase curvature is an unequivocal signature of a critical behaviour associated to a diverging correlation length. In retrospect, it might not come as a surprise that such a connection exists, as that is indeed what the intuition built up from the experience on equilibrium phenomena would suggest.

However, a similarly heuristic argument should point towards an analogous conclusion in the case of criticality defined by a closing dissipative gap. After all, this is the NESS-QPTs analogue of a vanishing Hamiltonian gap. One should legitimately expect that a closing dissipative gap, i.e. a critical slowing down, should result in a singular behaviour of the mean Uhlmann curvature. That this is a reasonable guess is further suggested by several studies on dissipative topological phase transitions [382], where topologically inequivalent regions of the phase diagram are separated by critical points with vanishing dissipative gap, which are not necessarily accompanied by a diverging correlation length in the bulk. However, one can analytically demonstrate that this second heuristic argument does not hold, showing that the Uhlmann curvature is sensitive to the criticality, but only in the sense of a truly diverging correlation length. In this sense, the Uhlmann 
curvature confirms its role as a witness of the purely kinematic aspects of the criticality, and it is only indirectly affected by the dynamical features of the NESS-QPTs.

Although the main focus of the review is on the physically relevant class of Fermionic quadratic models, this is by no means the only context in which this idea is applicable. This approach, for its generality, immediately extends to any equilibrium and non-equilibrium QPT, with and without order parameters, with or without symmetry breaking, including non-equilibrium dynamical phase-transitions, topological dissipative phase transitions, and cluster state phase transitions. Moreover, this idea is a promising tool which may glean insight on the interplay between competing orders both in equilibrium and non-equilibrium QPTs. It is hard to grasp the full extent of the implications that such a general approach may provide.

Going beyond the geometrical aspect mentioned in this review, the mean Uhlmann curvature and the Uhlmann geometric phase in general offer the possibility of studying topological structures on the manifold of density matrices. One can indeed formulate, under suitable assumption, a topologically nontrivial gauge structure based on the notion of mean Uhlmann curvature. In this framework, topological invariants that are protected by suitable symmetries may be identified for mixed states. One may define class of topologically inequivalent mappings from a parameter space into the density matrices which can be continuously deformed into each other only if the underlying symmetry assumptions are violated.

In a lattice translation-invariant system, one may think of identifying the parameter space with the Brillouin zone, thereby providing a possible way of generalising topological band structure invariants to the domain of mixed states. The possible applications of this conceptual framework is to obtain a topologically nontrivial Chern insulator or topological superconductors as a steady state of a non-equilibrium open quantum system.

Topological invariants, that may be constructed through the mean Uhlmann curvature, are in principle experimentally accessible via state tomography. However, a possible route of investigation would be to be able to construct a relation to natural observables such as response functions. A prototypical quantity to look at is the quantised Hall conductance. However, the formulation of a mixed state quantity, which under statistical mixture does not cause deviations from an integer quantisation of the Hall conductance, is quite an open challenge.

\section{Declaration of competing interest}

The authors declare that they have no known competing financial interests or personal relationships that could have appeared to influence the work reported in this paper.

\section{Acknowledgments}

This work was supported by the Grant of the Government Council on Grants, Russian Federation, contract No. 07402-2018-330 (2). We acknowledge also partial support by Ministry of Education, University and Research of the Italian Government.

\section{Appendix A. Fermionic Gaussian states}

We review here the main properties of Fermionic Gaussian States. Let us consider a system of $n$ Fermionic particles described by creation and annihilation operators $c_{j}^{\dagger}$ and $c_{j}$. These operators obey the canonical anticommutation relations,

$$
\left\{c_{j}, c_{k}\right\}=0 \quad\left\{c_{j}, c_{k}^{\dagger}\right\}=\delta_{j k} .
$$

Let us define the Hermitian Majorana operators as

$$
\omega_{2 j-1}:=c_{j}+c_{j}^{\dagger}, \quad \omega_{2 j}:=i\left(c_{j}-c_{j}^{\dagger}\right),
$$

which are generators of a Clifford algebra, and satisfy the following anti-commutation relations

$$
\left\{\omega_{j}, \omega_{k}\right\}=2 \delta_{j k} .
$$

Fermionic Gaussian states are defined as states that can be expressed as

$$
\rho=\frac{e^{-\frac{i}{4} \omega^{T} \Omega \omega}}{Z}, \quad Z:=\operatorname{Tr}\left[e^{-\frac{i}{4} \omega^{T} \Omega \omega}\right]
$$

where $\Omega$ is a $2 n \times 2 n$ real antisymmetric matrix. As any antisymmetric real matrix, $\Omega$ can be cast in the following canonical form by an orthogonal matrix $Q$, i.e.

$$
\Omega=Q^{T} \bigoplus_{k=1}^{n}\left(\begin{array}{cc}
0 & \Omega_{k} \\
-\Omega_{k} & 0
\end{array}\right) Q \quad Q^{T}=Q^{-1},
$$

where $\pm i \Omega_{k}$ are $\Omega$ 's eigenvalues. Let

$$
\boldsymbol{z}=\left(z_{1}, \ldots, z_{2 n}\right)^{T}:=Q \boldsymbol{\omega}
$$


be the vector of Majorana Fermions in the eigenmode representation. Hence,

$$
\begin{aligned}
& \rho=\frac{1}{Z} \prod_{k}\left[\cosh \left(\frac{\Omega_{k}}{2}\right)-i \sinh \left(\frac{\Omega_{k}}{2}\right) z_{2 k-1} z_{2 k}\right], \\
& Z=\prod_{k} 2 \cosh \left(\frac{\Omega_{k}}{2}\right) .
\end{aligned}
$$

Gaussian states are completely specified by the two-point correlation matrix

$$
\Gamma_{j k}:=1 / 2 \operatorname{Tr}\left(\rho\left[\omega_{j}, \omega_{k}\right]\right), \quad \Gamma=\Gamma^{\dagger}=-\Gamma^{T},
$$

which is an imaginary antisymmetric matrix. As

$$
\Gamma_{j k}=\frac{2 i}{Z} \frac{\partial Z}{\partial \Omega_{j k}},
$$

one can show that

$$
\Gamma=\tanh \left(i \frac{\Omega}{2}\right) \text {. }
$$

The correlation matrix is diagonal in the same basis of $\Omega$ and its eigenvalues read $\gamma_{k}=\tanh \left(\Omega_{k} / 2\right)$. Hence

$$
\rho=\prod_{k} \frac{1-i \gamma_{k} z_{2 k-1} z_{2 k}}{2},
$$

where $\left|\gamma_{k}\right| \leq 1$. Hence the Gaussian Fermionic state can be factorised into a tensor product $\rho=\bigotimes_{k} \rho_{k}$ of density matrices of the eigenmodes $\rho_{k}:=\frac{1-i \gamma_{k} z_{2 k-1} z_{2 k}}{2}$. Note that for $\gamma_{k}= \pm 1$, one has $\Omega_{k}= \pm \infty$, making the definition (A.4) of Gaussian state not well defined, unlike Eq. (A.11). Indeed, the latter offers an appropriate parameterisation even in those extremal points. Notice that $\left|\gamma_{k}\right|=1$ corresponds to a Fermionic mode $\tilde{c}_{k}=1 / 2\left(z_{2 k-1}+z_{2 k}\right)$ being in a pure state, as it is clear from the following explicit expression for the purity of the states $\rho_{k}$ :

$$
\begin{aligned}
& \operatorname{Tr}\left[\rho_{k}^{2}\right]=\frac{\operatorname{det}\left[2 \cosh \left(\Omega_{k}\right)\right]^{\frac{1}{2}}}{\operatorname{det}\left[2 \cosh \left(\frac{\Omega_{k}}{2}\right)\right]}, \\
& \operatorname{Tr}\left[\rho^{2}\right]=\frac{\operatorname{det}[2 \cosh (i \Omega)]^{\frac{1}{2}}}{\operatorname{det}\left[2 \cosh \left(i \frac{\Omega}{2}\right)\right]}=\sqrt{\operatorname{det}\left(\frac{1+\Gamma^{2}}{2}\right)} .
\end{aligned}
$$

\section{A.1. Symmetric logarithmic derivative for Fermionic Gaussian states}

We will review here useful expressions, adapted from Ref. [399], which are instrumental for the derivation of the symmetric logarithmic derivative of density matrices in the exponential form

$$
\rho=e^{D(\lambda)} .
$$

Clearly, a Gaussian Fermionic state can be expressed in the exponential form (A.14) by identifying

$$
D=-\frac{i}{4} \boldsymbol{w}^{\dagger} \cdot \Omega \cdot \boldsymbol{w}-\mathbb{1} \ln Z
$$

Notice, that the above parameterisation is well defined in the case of full-rank density matrices. As usual, the case of extremal conditions $\left|\gamma_{k}\right|=1$, where $\gamma_{k}$ is an eigenvalue of the correlation function, should be carried out as a limiting procedure.

The starting point is Eq. (2.1) of Ref. [401] for the derivative of density operators

$$
\partial_{\mu} \rho=\int_{0}^{1} e^{s D} \partial_{\mu} D e^{(1-s) D} d s
$$

One can use the nested-commutator relation

$$
\begin{aligned}
e^{D} A e^{-D} & =A+[D, A]+\frac{1}{2 !}[D,[D, A]]+\cdots \\
& =\sum_{n=0}^{\infty} \frac{1}{n !} \mathcal{C}^{n}(A)=e^{\mathcal{C}}(A)
\end{aligned}
$$


where $\mathcal{C}^{n}(A)$, a linear operation on $A$, denotes the $n$ th-order nested commutator $[D, \ldots,[D, A]]$, with $\mathcal{C}^{0}(A)=A$. Applying this relation to the expression (A.16) leads to

$$
\begin{aligned}
\partial_{\mu} \rho \rho^{-1} & =\partial_{\mu} D+\frac{1}{2 !}\left[D, \partial_{\mu} D\right]+\frac{1}{3 !}\left[D,\left[D, \partial_{\mu} D\right]\right]+\cdots \\
& =\sum_{n=0}^{\infty} \frac{1}{(n+1) !} \mathcal{C}^{n}\left(\partial_{\mu} D\right)=h(\mathcal{C})\left(\partial_{\mu} D\right),
\end{aligned}
$$

where $h$ is the generating function of the expansion coefficients in Eq. (A.17),

$$
h(t)=1+\frac{t}{2 !}+\frac{t^{2}}{3 !}+\cdots=\frac{e^{t}-1}{t} .
$$

Using the definition of symmetric logarithmic derivative, i.e.

$$
\partial_{\mu} \rho=\frac{1}{2}\left(L_{\mu} \rho+\rho L_{\mu}\right),
$$

and that of density matrix in exponential form (A.14), one gets

$$
\begin{aligned}
\partial_{\mu} \rho \rho^{-1} & =\frac{1}{2}\left(L+e^{D} L e^{-D}\right) \\
& =\frac{1}{2}\left(L+\sum_{n=0}^{\infty} \frac{1}{n !} \mathcal{C}^{n}(L)\right)=r(\mathcal{C})(L),
\end{aligned}
$$

where the generating function is $r(t)=\left(e^{t}+1\right) / 2$. Suppose that the SLD adopts the form,

$$
L_{\mu}=\sum_{n=0}^{\infty} f_{n} \mathcal{C}^{n}\left(\partial_{\mu} D\right)=f(\mathcal{C})\left(\partial_{\mu} D\right),
$$

with the generating function

$$
f(t)=f_{0}+f_{1} t+f_{2} t^{2}+\cdots
$$

to be determined. Plugging Eq. (A.21) into Eq. (A.20) yields

$$
\partial_{\mu} \rho \rho^{-1}=r(\mathcal{C})\left[f(\mathcal{C})\left(\partial_{\mu} D\right)\right]=r \circ f(\mathcal{C})\left(\partial_{\mu} D\right)=r \cdot f(\mathcal{C})\left(\partial_{\mu} D\right),
$$

where the identity $r \circ f=r \cdot f$ between the combination and the simple product of the two functions arises from $\mathcal{C}^{n}\left(\mathcal{C}^{m}(A)\right)=\mathcal{C}^{n+m}(A)$. Comparing Eq. (A.23) with Eq. (A.17) leads to the following relation between generating functions,

$$
f(t)=\frac{h(t)}{r(t)}=\frac{\tanh (t / 2)}{t / 2}=\sum_{n=0}^{\infty} \frac{4\left(4^{n+1}-1\right) B_{2 n+2}}{(2 n+2) !} t^{2 n},
$$

where $B_{2 n+2}$ is the $(2 n+2)$ th Bernoulli number. Comparing Eqs. (A.22) with (A.24), we have

$$
f_{n}= \begin{cases}\frac{4\left(4^{n / 2+1}-1\right) B_{n+2}}{(n+2) !}, & \text { for even } n, \\ 0, & \text { for odd } n .\end{cases}
$$

The vanishing of the odd-order $f_{n}$ 's is a consequence of the Hermiticity of $L$, which makes $f(t)$ an even function.

For a Gaussian Fermionic state the operator $D$ in terms of the Majorana Fermions of the eigenmodes is written

$$
D=-\frac{i}{4} \sum_{k} \Omega_{k}\left[z_{2 k-1}, z_{2 k}\right]-\mathbb{1} \ln Z=\sum_{k} \Omega_{k}\left(\tilde{c}_{k}^{\dagger} \tilde{c}_{k}-\frac{1}{2}\right)-\mathbb{1} \ln Z
$$

where $\tilde{c}_{k}:=\frac{1}{2}\left(z_{2 k-1}+i z_{2 k}\right), \tilde{c}_{k}^{\dagger}:=\frac{1}{2}\left(z_{2 k-1}-i z_{2 k}\right)$ are the ordinary annihilation and creation operators of the eigenmodes. It is straightforward to derive the commutation relations between $D$ and Fermionic operators,

$$
\left[D, \tilde{c}_{k}\right]=-\Omega_{k} \tilde{c}_{k}, \quad\left[D, \tilde{c}_{k}^{\dagger}\right]=\Omega_{k} \tilde{c}_{k}^{\dagger},
$$

and for quadratic operators, we get also

$$
\left[D, \tilde{c}_{j}^{\dagger} \tilde{c}_{k}\right]=\left(\Omega_{j}-\Omega_{k}\right) \tilde{c}_{j}^{\dagger} \tilde{c}_{k}, \quad\left[D, \tilde{c}_{j} \tilde{c}_{k}\right]=\left(\Omega_{j}-\Omega_{k}\right) \tilde{c}_{j} \tilde{c}_{k} .
$$

Consequently, one finds

$$
\begin{aligned}
f(\mathcal{C})\left(\tilde{c}_{k}^{\dagger} \tilde{c}_{k}\right) & =f\left(\Omega_{k}-\Omega_{k}\right) \tilde{c}_{k}^{\dagger} \tilde{c}_{k}, \\
f(\mathcal{C})\left(\tilde{c}_{k} \tilde{c}_{k}\right) & =f\left(\Omega_{k}+\Omega_{k}\right) \tilde{c}_{k} \tilde{c}_{k} .
\end{aligned}
$$


Most generally, the derivative of $D$ takes the form

$$
\partial_{\mu} D=-\frac{1}{2} \boldsymbol{c} \partial_{\mu} \Omega^{\prime} \boldsymbol{c}-\frac{\partial_{\mu} Z}{Z},
$$

which plugged into formula (A.21) shows that $L_{\mu}$ is at most quadratic in Fermionic operators.

\section{Appendix B. Spectral properties of Quadratic Liouvillians}

We will review the main results on the spectral properties of the quadratic Fermionic Liouvillian (191). Note that the real matrix $X$ defined in (211) has no general structure apart from the fact that $X+X^{T}=8 \operatorname{Re} M \geq 0$, where $M$ is the bath matrix defined in (213), whose positive semi-definiteness implies $\mathbf{R e} M \geq 0$. We will drop here the assumption made in Section 15, about the diagonalisability of $X$, and will show that the qualitative aspects of the results derived in Section 16 still hold.

The matrix $X$ can always be put in the Jordan canonical form, i.e. $X=U D_{X}^{J} U^{-1}$ with $D_{X}^{J}=\oplus_{m} J_{\ell_{m}}\left(x_{m}\right)$,

$$
J_{\ell_{m}}\left(x_{m}\right)=\left(\begin{array}{ccccc}
x_{m} & 1 & & & \\
& x_{m} & 1 & & \\
& & x_{m} & 1 & \\
& & & \ddots & \ddots
\end{array}\right),
$$

where $x_{m}$ are (possibly equal) eigenvalues of $X$ and $\ell_{m}$ is the dimension of the Jordan block: each block is composed of $\ell_{m}$ degenerate eigenvalues of $X$. The form of the transformation (223) remains the same (although with a new matrix $U$ ) while (227) becomes

$$
\mathcal{L}=-\sum_{j=1}^{2 n} x_{j} \hat{b}_{j}^{\times} \hat{b}_{j}-\sum_{m} \sum_{k=1}^{\ell_{m}-1} \hat{b}_{m_{k}+1}^{\times} \hat{b}_{m_{k}},
$$

where $m_{k}$ refers to the index of the $k$ th element in the $m$ th Jordan block. It is clear that the state (230) is still a stationary state.

Lemma 3. Let $X$ be a real square matrix, such that $X+X^{T} \geq 0$. Then:

(i) Any eigenvalue $x_{j}$ of $X$ satisfies $\boldsymbol{R e}_{j} \geq 0$.

(ii) For any eigenvalue $x_{j}$ of $X$ on the imaginary line, $\boldsymbol{R} \mathbf{e} x_{j}=0$, its algebraic and geometric multiplicities coincide.

Proof. (i) Let $x_{j}$ be an eigenvalue and let $\boldsymbol{u}_{j}$ be its corresponding eigenvector. One can write $X \boldsymbol{u}_{j}=x_{j} \boldsymbol{u}_{j}$, and the complex conjugate of this equation $X \boldsymbol{u}^{*}=x_{j}^{*} \boldsymbol{u}^{*}$. Then take a scalar product of the first equation with $\boldsymbol{u}^{\dagger}$ and the scalar product of the second equation with $\boldsymbol{u}$ and sum up:

$$
\boldsymbol{u}^{\dagger} \cdot\left(X+X^{T}\right) \boldsymbol{u}=\left(2 \operatorname{Re} x_{j}\right) \boldsymbol{u}^{\dagger} \cdot \boldsymbol{u} .
$$

Strict positivity of the eigenvector norm, $\boldsymbol{u}^{\dagger} \cdot \boldsymbol{u}>0$, and the positive semi-definiteness, $\left(X+X^{T}\right) \geq 0$, imply $\operatorname{Re} x_{j} \geq 0$.

(ii) Consider a linear system of differential equations,

$$
(d / d t) \boldsymbol{u}(t)=-X \boldsymbol{u}(t)
$$

Positive semi-definiteness of $X+X^{T}$ is equivalent to Lyapunov stability in control theory, namely

$$
(d / d t)\|\boldsymbol{u}\|_{2}^{2}=-\overline{\boldsymbol{u}} \cdot\left(X+X^{T}\right) \boldsymbol{u} \leq 0 \quad \text { iff } \quad X+X^{T} \geq 0 .
$$

Then, one can show that, in order for the system (B.4) to be Lyapunov stable, a purely imaginary (or vanishing) eigenvalue $x_{m}=i b$ cannot correspond to a Jordan block of dimension $\ell_{m}>1$ in the Jordan canonical form of $X$. This is indeed obvious since, if we take the initial vector $\boldsymbol{u}(0)$ for (B.4) from $\operatorname{ker}\left(X-x_{m} \mathbb{1}\right)^{\ell_{m}} \ominus \operatorname{ker}\left(X-x_{m} \mathbb{1}\right)^{\ell_{m}-1}$, then $\boldsymbol{u}(t) \propto t^{\ell_{m}-1} e^{-i b t}$. Hence, if the system (B.4) is not Lyapunov stable, $X+X^{T} \geq 0$.

In [390] it has been shown that the spectrum of the Liouvillian is

$$
\operatorname{Sp}(\mathcal{L})=-\left\{x_{\mathbf{n}}:=\sum_{m} x_{m} n_{m} / n_{m}=0, \ldots, \ell_{m}\right\} .
$$

Accordingly, $\Delta_{\mathcal{L}}=\Delta \equiv 2 \min _{m} \operatorname{Re}\left[x_{m}\right.$ ]. If $\Delta>0$ the steady state (230) is unique [390].

In the non-diagonalisable case the last equation in (241) is not satisfied. On the other hand one can obtain the following [177] 


\section{Proposition 4.}

$$
\left\|\hat{X}^{-1}\right\|_{\infty}<\frac{1+p\left(\Delta^{-1}\right)}{\Delta},
$$

for a certain polynomial $p()$.

Proof. We start by writing

$$
\begin{aligned}
\hat{X} & =\bigoplus_{m} J_{\ell_{m}}\left(x_{m}\right) \otimes \mathbb{1}+\bigoplus_{m} \mathbb{1} \otimes J_{\ell_{m}}\left(x_{m}\right) \\
& =\bigoplus_{m, n}\left[J_{\ell_{m}}\left(x_{m}\right) \otimes \mathbb{1}_{\ell_{n}}+\mathbb{1}_{\ell_{m}} \otimes J_{\ell_{n}}\left(x_{n}\right)\right] \\
& =\hat{x}+\bigoplus_{m, n}\left[J_{\ell_{m}}(0) \otimes \mathbb{1}_{\ell_{n}}+\mathbb{1}_{\ell_{m}} \otimes J_{\ell_{n}}(0)\right],
\end{aligned}
$$

where $D_{\hat{x}}$ is the diagonal matrix with entries $x_{i}+x_{j}$ and where we used the decomposition $\mathbb{1}=\oplus_{m} 1_{\ell_{m}}$. Moreover, thanks to Lemma 3.1 of Ref. [390],

$$
\begin{aligned}
\hat{X} & =D_{\hat{X}}+\bigoplus_{m, n} \bigoplus_{r=1}^{\min \left\{\ell_{m}, \ell_{n}\right\}} J_{\ell_{m}+\ell_{n}-2 r+1}(0) \\
& =D_{\hat{X}}\left[\mathbb{1}+\bigoplus_{m, n} \bigoplus_{r=1}^{\min \left\{\ell_{m}, \ell_{n}\right\}} \frac{J_{\ell_{m}+\ell_{n}-2 r+1}(0)}{x_{m}+x_{n}}\right] .
\end{aligned}
$$

As $J$ is nilpotent,

$$
\hat{X}^{-1}=D_{\hat{X}}^{-1}\left[\mathbb{1}+\bigoplus_{m, n} \bigoplus_{r=1}^{\min \left\{\ell_{m}, \ell_{n}\right\}} \sum_{m=1}^{\ell_{m}+\ell_{n}-2 r}\left(-\frac{J_{\ell_{m}+\ell_{n}-2 r+1}(0)}{x_{m}+x_{n}}\right)^{m}\right],
$$

and

$$
\begin{aligned}
\left\|\hat{X}^{-1}\right\|_{\infty} & \leq\left\|D_{\hat{X}}^{-1}\right\|_{\infty}\left[1+\max _{m, n} \max _{r} \sum_{m=1}^{\ell_{m}+\ell_{n}-2 r} \frac{1}{\left|x_{m}+x_{n}\right|^{m}}\right] \\
& =\left\|D_{\hat{X}}^{-1}\right\|_{\infty}\left[1+\max _{m, n} \sum_{m=1}^{\ell_{m}+\ell_{n}-2} \frac{1}{\left|x_{m}+x_{n}\right|^{m}}\right] \\
& \leq \frac{1}{\Delta}\left[1+\max _{m, n} \sum_{m=1}^{\ell_{m}+\ell_{n}-2} \frac{1}{\Delta^{m}}\right] .
\end{aligned}
$$

\section{References}

[1] M. Greiner, O. Mandel, T. Esslinger, T.W. Hänsch, I. Bloch, Quantum phase transition from a superfluid to a Mott insulator in a gas of ultracold atoms, Nature 415 (6867) (2002) 39-44, http://dx.doi.org/10.1038/415039a, URL http://www.nature.com/doifinder/10.1038/415039a.

[2] H. Nishimori, G. Ortiz, Elements of Phase Transitions and Critical Phenomena, Oxford University Press, 2010, http://dx.doi.org/10. 1093/acprof:oso/9780199577224.001.0001, URL http://www.oxfordscholarship.com/view/10.1093/acprof:oso/9780199577224.001.0001/acprof9780199577224.

[3] G. Mussardo, Statistical Field Theory : An Introduction to Exactly Solved Models in Statistical Physics, Oxford University Press, 2010, p. 755, URL https://global.oup.com/academic/product/statistical-field-theory-9780199547586?cc=it\&lang=en\&.

[4] P.M. Chaikin, T.C. Lubensky, Principles of Condensed Matter Physics, Cambridge University Press, 1995, p. 699.

[5] N. Goldenfeld, Lectures on Phase Transitions and the Renormalization Group, Addison-Wesley, Advanced Book Program, 1992,1992, p. 394.

[6] H.E.H.E. Stanley, Introduction to Phase Transitions and Critical Phenomena, Oxford University Press, 1987, p. 308.

[7] S. Sachdev, Quantum Phase Transitions, Cambridge University press, 2011, p. 501.

[8] S.L. Sondhi, S.M. Girvin, J.P. Carini, D. Shahar, Continuous quantum phase transitions, Rev. Modern Phys. 69 (1) (1997) 315-333, http: //dx.doi.org/10.1103/RevModPhys.69.315, URL https://link.aps.org/doi/10.1103/RevModPhys.69.315.

[9] M. Vojta, Quantum phase transitions, Rep. Progr. Phys. 66 (12) (2003) 2069-2110, http://dx.doi.org/10.1088/0034-4885/66/12/R01, URL http://stacks.iop.org/0034-4885/66/i=12/a=R01?key=crossref.c536678c9f7b611291c4445db9c68861.

[10] D. Belitz, T. Vojta, How generic scale invariance influences quantum and classical phase transitions, Rev. Modern Phys. 77 (2) (2005) 579-632, http://dx.doi.org/10.1103/RevModPhys.77.579, URL https://link.aps.org/doi/10.1103/RevModPhys.77.579.

[11] L. Carr, Understanding Quantum Phase Transitions, CRC Press, 2011, p. 728.

[12] S. Suzuki, J.-i. Inoue, B.K. Chakrabarti, Quantum Ising Phases and Transitions in Transverse Ising Models, in: Lecture Notes in Physics, vol. 862, Springer Berlin Heidelberg, Berlin, Heidelberg, 2013, http://dx.doi.org/10.1007/978-3-642-33039-1, URL http://link.springer.com/10.1007/9783-642-33039-1.

[13] K. Wilson, The renormalization group and the $\epsilon$ expansion, Phys. Rep. 12 (2) (1974) 75-199, http://dx.doi.org/10.1016/0370-1573(74)90023-4, URL https://linkinghub.elsevier.com/retrieve/pii/0370157374900234. 
[14] G. Parisi, Statistical field theory, Addison-Wesley Pub. Co, 1988, p. 352.

[15] J. Zinn-Justin, Quantum Field Theory and Critical Phenomena, Oxford University Press, 2002, http://dx.doi.org/10.1093/acprof:oso/ 9780198509233.001.0001, URL http://www.oxfordscholarship.com/view/10.1093/acprof:oso/9780198509233.001.0001/acprof-9780198509233.

[16] J.L. Cardy, Scaling and Renormalization in Statistical Physics, Cambridge University Press, 1996, p. 238.

[17] A. Uhlmann, The "transition probability" in the state space of a *-algebra, Reports Math. Phys. 9 (2) (1976) 273-279, http://dx.doi.org/10. 1016/0034-4877(76)90060-4, URL http://linkinghub.elsevier.com/retrieve/pii/0034487776900604.

[18] P.M. Alberti, A. Uhlmann, Stochastic linear maps and transition probability, Lett. Math. Phys. 7 (2) (1983) 107-112, http://dx.doi.org/10.1007/ BF00419927, URL http://link.springer.com/10.1007/BF00419927.

[19] P.M. Alberti, A note on the transition probability over C*-algebras, Lett. Math. Phys. 7 (1) (1983) 25-32, http://dx.doi.org/10.1007/BF00398708, URL http://link.springer.com/10.1007/BF00398708.

[20] P. Alberti, A. Uhlmann, Transition probabilities on W and C algebras, in: H. Baumgärtel, A.U. G. Laßner, A. Pietsch (Eds.), Proc. Second Int. Conf. Oper. Algebr. Ideals, Their Appl. Theor. Phys., in: Teubner Texte zur Mathematik, BSB B. G. Teubner-Verl., Leipzig, 1984, pp. 5-11.

[21] W.K. Wootters, Statistical distance and Hilbert space, Phys. Rev. D 23 (2) (1981) 357-362, http://dx.doi.org/10.1103/PhysRevD.23.357, URL https://link.aps.org/doi/10.1103/PhysRevD.23.357.

[22] R. Jozsa, Fidelity for mixed quantum states, J. Modern Opt. 41 (12) (1994) 2315-2323, http://dx.doi.org/10.1080/09500349414552171, URL http://www.tandfonline.com/doi/abs/10.1080/09500349414552171.

[23] B. Schumacher, Quantum coding, Phys. Rev. A 51 (4) (1995) 2738-2747, http://dx.doi.org/10.1103/PhysRevA.51.2738, URL https://link.aps.org/ doi/10.1103/PhysRevA.51.2738.

[24] C.A. Fuchs, Distinguishability and Accessible Information in Quantum Theory (Ph.D. thesis), University of New Mexico, Albuquerque, 1996, URL http://arxiv.org/abs/quant-ph/9601020.

[25] D. Bures, An extension of Kakutani's theorem on infinite product measures to the tensor product of semifinite w*-algebras, Trans. Amer. Math. Soc. 135 (5) (1969) 199, http://dx.doi.org/10.1090/S0002-9947-1969-0236719-2, URL http://www.ams.org/jourcgi/jour-getitem?pii=S00029947-1969-0236719-2.

[26] M. Nakahara, Geometry, Topology and Physics, in: Graduate Student Series in Physics, Adam Hilger, Bristol and New York, 1990.

[27] M.V. Berry, Quantal phase factors accompanying adiabatic changes, Proc. R. Soc. Lond. Ser. A Math. Phys. Eng. Sci. 392 (1802) (1984) 45-57, http://dx.doi.org/10.1098/rspa.1984.0023, URL http://rspa.royalsocietypublishing.org/cgi/doi/10.1098/rspa.1984.0023.

[28] M.V. Berry, The quantum phase, five years after, in: Geom. PHASES Phys., World Scientific (Singapore), 1989, pp. 7-28, http://dx.doi.org/10. 1142/9789812798381_0001.

[29] F. Wilczek, A. Shapere, Geometric Phases in Physics, in: Advanced Series in Mathematical Physics, vol. 5, WORLD SCIENTIFIC, 1989, http://dx.doi.org/10.1142/0613, URL https://www.worldscientific.com/worldscibooks/10.1142/0613.

[30] A. Bohm, A. Mostafazadeh, H. Koizumi, Q. Niu, J. Zwanziger, The Geometric Phase in Quantum Systems, Springer Berlin Heidelberg, Berlin, Heidelberg, 2003, http://dx.doi.org/10.1007/978-3-662-10333-3, URL http://link.springer.com/10.1007/978-3-662-10333-3.

[31] A.C.M. Carollo, J.K. Pachos, Geometric phases and criticality in spin-chain systems, Phys. Rev. Lett. 95 (15) (2005) 157203, http://dx.doi.org/ 10.1103/PhysRevLett.95.157203, URL https://link.aps.org/doi/10.1103/PhysRevLett.95.157203.

[32] A. Hamma, Berry phases and quantum phase transitions, 2006.

[33] S.-L. Zhu, Geometric phases and quantum phase transitions, Internat. J. Modern Phys. B 22 (06) (2008) 561-581, http://dx.doi.org/10.1142/ S0217979208038855, URL http://www.worldscientific.com/doi/abs/10.1142/S0217979208038855.

[34] D.J. Thouless, Quantization of particle transport, Phys. Rev. B 27 (10) (1983) 6083-6087, http://dx.doi.org/10.1103/PhysRevB.27.6083.

[35] B.A. Bernevig, T.L. Hughes, Topological Insulators and Topological Superconductors, Princeton University Press, 2013.

[36] C.-K. Chiu, J.C.Y. Teo, A.P. Schnyder, S. Ryu, Classification of topological quantum matter with symmetries, Rev. Modern Phys. 88 (3) (2016) 035005, http://dx.doi.org/10.1103/RevModPhys.88.035005, URL https://link.aps.org/doi/10.1103/RevModPhys.88.035005.

[37] J.K. Pachos, A.C. Carollo, Geometric phases and criticality in spin systems, Phil. Trans. R. Soc. A 364 (1849) (2006) 3463-3476, http: //dx.doi.org/10.1098/rsta.2006.1894, URL http://www.royalsocietypublishing.org/doi/10.1098/rsta.2006.1894.

[38] F. Plastina, G. Liberti, A. Carollo, Scaling of Berry's phase close to the Dicke quantum phase transition, Europhys. Lett. 76 (2) (2006) 182-188, http://dx.doi.org/10.1209/epl/i2006-10270-x, URL http://stacks.iop.org/0295-5075/76/i=2/a=182?key=crossref 35948bee527d447741539fbe9a87bddc.

[39] S.-1. Zhu, Scaling of geometric phases close to the quantum phase transition in the XY spin chain, Phys. Rev. Lett. 96 (7) (2006) 077206, http://dx.doi.org/10.1103/PhysRevLett.96.077206, URL https://link.aps.org/doi/10.1103/PhysRevLett.96.077206.

[40] M.E. Reuter, M.J. Hartmann, M.B. Plenio, Geometric phases and critical phenomena in a chain of interacting spins, Proc. R. Soc. A Math. Phys. Eng. Sci. 463 (2081) (2007) 1271-1285, http://dx.doi.org/10.1098/rspa.2007.1822, URL http://www.royalsocietypublishing.org/doi/10.1098/rspa. 2007.1822.

[41] X. Peng, S. Wu, J. Li, D. Suter, J. Du, Observation of the ground-state geometric phase in a heisenberg XY model, Phys. Rev. Lett. 105 (24) (2010) 240405, http://dx.doi.org/10.1103/PhysRevLett.105.240405, URL https://link.aps.org/doi/10.1103/PhysRevLett.105.240405.

[42] L. Campos Venuti, P. Zanardi, Quantum critical scaling of the geometric tensors, Phys. Rev. Lett. 99 (9) (2007) 095701, http://dx.doi.org/10. 1103/PhysRevLett.99.095701, URL https://link.aps.org/doi/10.1103/PhysRevLett.99.095701.

[43] H. Cui, K. Li, X. Yi, Geometric phase and quantum phase transition in the Lipkin-Meshkov-Glick model, Phys. Lett. A 360 (2) (2006) 243-248, http://dx.doi.org/10.1016/j.physleta.2006.08.040, URL https://linkinghub.elsevier.com/retrieve/pii/S0375960106012795.

[44] G. Chen, J. Li, J.-Q. Liang, Critical property of the geometric phase in the Dicke model, Phys. Rev. A 74 (5) (2006) 054101, http://dx.doi.org/ 10.1103/PhysRevA.74.054101, URL https://link.aps.org/doi/10.1103/PhysRevA.74.054101.

[45] X.X. Yi, W. Wang, Geometric phases induced in auxiliary qubits by many-body systems near their critical points, Phys. Rev. A 75 (3) (2007) 032103, http://dx.doi.org/10.1103/PhysRevA.75.032103, URL https://link.aps.org/doi/10.1103/PhysRevA.75.032103.

[46] Z.-G. Yuan, P. Zhang, S.-S. Li, Loschmidt echo and Berry phase of a quantum system coupled to an XY spin chain: Proximity to a quantum phase transition, Phys. Rev. A 75 (1) (2007) 012102, http://dx.doi.org/10.1103/PhysRevA.75.012102, URL https://link.aps.org/doi/10.1103/PhysRevA. 75.012102 .

[47] H.T. Cui, J. Yi, Geometric phase and quantum phase transition: Two-band model, Phys. Rev. A 78 (2) (2008) 022101, http://dx.doi.org/10.1103/ PhysRevA.78.022101, URL https://link.aps.org/doi/10.1103/PhysRevA.78.022101.

[48] C. Furtado, F. Moraes, A.M. de M. Carvalho, Geometric phases in graphitic cones, Phys. Lett. A 372 (32) (2008) 5368-5371, http://dx.doi.org/ 10.1016/j.physleta.2008.06.029, URL https://linkinghub.elsevier.com/retrieve/pii/S0375960108009249.

[49] M.-G. Hu, K. Xue, M.-L. Ge, Exact solution of a Yang-Baxter spin- 1/2 chain model and quantum entanglement, Phys. Rev. A 78 (5) (2008) 052324, http://dx.doi.org/10.1103/PhysRevA.78.052324, URL https://link.aps.org/doi/10.1103/PhysRevA.78.052324.

[50] A.I. Nesterov, S.G. Ovchinnikov, Geometric phases and quantum phase transitions in open systems, Phys. Rev. E 78 (1) (2008) 015202 http://dx.doi.org/10.1103/PhysRevE.78.015202, URL https://link.aps.org/doi/10.1103/PhysRevE.78.015202.

[51] N. Paunković, V. Rocha Vieira, Macroscopic distinguishability between quantum states defining different phases of matter: Fidelity and the uhlmann geometric phase, Phys. Rev. E 77 (1) (2008) 011129, http://dx.doi.org/10.1103/PhysRevE.77.011129, URL https://link.aps.org/doi/10. 1103/PhysRevE.77.011129. 
[52] H.A. Contreras, A.F. Reyes-Lega, Berry phases, quantum phase transitions and chern numbers, Phys. B Condens. Matter 403 (5-9) (2008) 1301-1302, http://dx.doi.org/10.1016/j.physb.2007.10.131, URL https://linkinghub.elsevier.com/retrieve/pii/S0921452607011313.

[53] Y.-Q. Ma, S. Chen, Geometric phase and quantum phase transition in an inhomogeneous periodic XY spin-1/2 model, Phys. Rev. A 79 (2) (2009) 022116, http://dx.doi.org/10.1103/PhysRevA.79.022116, URL https://link.aps.org/doi/10.1103/PhysRevA.79.022116.

[54] S. Oh, Geometric phases and entanglement of two qubits with XY type interaction, Phys. Lett. A 373 (6) (2009) 644-647, http://dx.doi.org/ 10.1016/j.physleta.2008.12.023, URL https://linkinghub.elsevier.com/retrieve/pii/S0375960108017635.

[55] A.I. Nesterov, S.G. Ovchinnikov, Spin crossover: the quantum phase transition induced by high pressure, JETP Lett. 90 (7) (2009) 530-534, http://dx.doi.org/10.1134/S0021364009190072, URL http://link.springer.com/10.1134/S0021364009190072.

[56] H.T. Cui, Y.F. Zhang, Pairwise entanglement and geometric phase in high dimensional free-Fermion lattice systems, Eur. Phys. J. D 51 (3) (2009) 393-400, http://dx.doi.org/10.1140/epjd/e2009-00025-9, URL http://www.springerlink.com/index/10.1140/epjd/e2009-00025-9.

[57] H.T. Quan, Finite-temperature scaling of magnetic susceptibility and the geometric phase in the XY spin chain, J. Phys. A Math. Theory 42 (39) (2009) 395002, http://dx.doi.org/10.1088/1751-8113/42/39/395002, URL http://stacks.iop.org/1751-8121/42/i=39/a=395002?key=crossref. 727dcff978dbbffece51ab7b0bf75b51.

[58] G. Wang, K. Xue, C. Sun, T. Hu, C. Zhou, G. Du, Quantum phase transition like phenomenon in a two-qubit Yang-Baxter system, Internat. J. Theoret. Phys. 49 (10) (2010) 2499-2505, http://dx.doi.org/10.1007/s10773-010-0435-x, URL http://link.springer.com/10.1007/s10773-0100435- $\mathrm{x}$.

[59] L.C. Wang, X.X. Yi, Geometric phase and quantum phase transition in the one-dimensional compass model, Eur. Phys. J. D 57 (2) (2010) 281-286, http://dx.doi.org/10.1140/epjd/e2010-00045-4, URL http://www.springerlink.com/index/10.1140/epjd/e2010-00045-4.

[60] E. Sjöqvist, R. Rahaman, U. Basu, B. Basu, Berry phase and fidelity susceptibility of the three-qubit Lipkin-Meshkov-Glick ground state, J. Phys. A Math. Theory 43 (35) (2010) 354026, http://dx.doi.org/10.1088/1751-8113/43/35/354026, URL http://stacks.iop.org/1751-8121/43/i= 35/a=354026?key=crossref.e3039e95c0afd658c7946ea2d515a0e5.

[61] B. Basu, P. Bandyopadhyay, The geometric phase and the dynamics of quantum phase transition induced by a linear quench, J. Phys. A Math. Theory 43 (35) (2010) 354023, http://dx.doi.org/10.1088/1751-8113/43/35/354023, URL http://stacks.iop.org/1751-8121/43/i=35/a=354023? key=crossref.8f9a51bb9ed5af39414ee38c5d21f5d4.

[62] X.-M. Lu, X. Wang, Operator quantum geometric tensor and quantum phase transitions, EPL (Europhys. Lett. 91 (3) (2010) 30003, http://dx. doi.org/10.1209/0295-5075/91/30003, URL http://stacks.iop.org/0295-5075/91/i=3/a=30003?key=crossref.a621f08a017adfa346a410d3c33a93ba.

[63] L. Zhi-Jian, C. Lu, W. Jiao-Jin, Critical entanglement and geometric phase of a two-qubit model with Dzyaloshinski-Moriya anisotropic interaction, Chin. Phys. B 19 (1) (2010) http://dx.doi.org/10.1088/1674-1056/19/1/010305, URL http://stacks.iop.org/1674-1056/19/i=1/a= 010305? key=crossref.8fba5aab45e5735584280bec0cfe8b43.

[64] B. Basu, Dynamics of the geometric phase in the adiabatic limit of a quench induced quantum phase transition, Phys. Lett. A 374 (10) (2010) 1205-1208, http://dx.doi.org/10.1016/j.physleta.2009.12.072, URL https://linkinghub.elsevier.com/retrieve/pii/S037596011000006X.

[65] M. Zhong, P. Tong, The ising and anisotropy phase transitions of the periodic XY model in a transverse field, J. Phys. A Math. Theory 43 (50) (2010) 505302, http://dx.doi.org/10.1088/1751-8113/43/50/505302, URL http://stacks.iop.org/1751-8121/43/i=50/a=505302?key=crossref. 785360de7a2d61cfc0a1b8e8747a50ff.

[66] F.M. Cucchietti, J.-F. Zhang, F.C. Lombardo, P.I. Villar, R. Laflamme, Geometric phase with nonunitary evolution in the presence of a quantum critical bath, Phys. Rev. Lett. 105 (24) (2010) 240406, http://dx.doi.org/10.1103/PhysRevLett.105.240406, URL https://link.aps.org/doi/10.1103/ PhysRevLett.105.240406.

[67] X.-Z. Yuan, H.-S. Goan, K.-D. Zhu, Geometric phase of a central spin coupled to an antiferromagnetic environment, Phys. Rev. A 81 (3) (2010) 034102, http://dx.doi.org/10.1103/PhysRevA.81.034102, URL https://link.aps.org/doi/10.1103/PhysRevA.81.034102.

[68] W. Cheng, C. Shan, Y. Huang, T. Liu, H. Li, Geometric phase signature of quantum criticality in the XY spin chain with multiple interaction, Phys. B Condens. Matter 405 (23) (2010) 4821-4824, http://dx.doi.org/10.1016/j.physb.2010.09.012, URL https://linkinghub.elsevier.com/retrieve/pii/ S092145261000880X.

[69] P. Bandyopadhyay, Anisotropic spin system, quantized Dirac monopole and the Berry phase, Proc. R. Soc. A Math. Phys. Eng. Sci. 467 (2126) (2011) 427-438, http://dx.doi.org/10.1098/rspa.2010.0266, URL http://www.royalsocietypublishing.org/doi/10.1098/rspa.2010.0266.

[70] F. Ribeiro, J. de Lima, L. Gonçalves, Quantum phase transitions of the extended isotropic XY model with long-range interactions, J. Magn. Magn. Mater. 323 (1) (2011) 39-50, http://dx.doi.org/10.1016/j.jmmm.2010.08.027, URL https://linkinghub.elsevier.com/retrieve/pii/ S0304885310005664.

[71] H. Lian, D. Tian, Quantum phase transition in XY spin chain with three-site interaction studied in terms of Loschmidt echo and Berry phase, Phys. Lett. A 375 (41) (2011) 3604-3609, http://dx.doi.org/10.1016/j.physleta.2011.08.025, URL https://linkinghub.elsevier.com/retrieve/ pii/S0375960111009984.

[72] L.-J. Tian, C.-Q. Zhu, H.-B. Zhang, L.-G. Qin, Fidelity susceptibility and geometric phase in critical phenomenon, Chin. Phys. B 20 (4) (2011) 040302, http://dx.doi.org/10.1088/1674-1056/20/4/040302, URL http://stacks.iop.org/1674-1056/20/i=4/a=040302?key=crossref. 000b4c635960dcc1fee133904cfc8ccc.

[73] S.-C. Li, L.-B. Fu, Quantum phase transition from mixed atom-molecule phase to pure molecule phase: Characteristic scaling laws and Berrycurvature signature, Phys. Rev. A 84 (2) (2011) 023605, http://dx.doi.org/10.1103/PhysRevA.84.023605, URL https://link.aps.org/doi/10.1103/ PhysRevA.84.023605.

[74] S.C. Li, J. Liu, L.B. Fu, Berry phase and hannay angle of an interacting boson system, Phys. Rev. A 83 (4) (2011) 042107, http://dx.doi.org/10. 1103/PhysRevA.83.042107, URL https://link.aps.org/doi/10.1103/PhysRevA.83.042107.

[75] C.S. Castro, M.S. Sarandy, Entanglement dynamics via geometric phases in quantum spin chains, Phys. Rev. A 83 (4) (2011) 042334, http://dx.doi.org/10.1103/PhysRevA.83.042334, URL https://link.aps.org/doi/10.1103/PhysRevA.83.042334.

[76] A. Patra, V. Mukherjee, A. Dutta, Path-dependent scaling of geometric phase near a quantum multi-critical point, J. Stat. Mech. Theory Exp. 2011 (03) (2011) P03026, http://dx.doi.org/10.1088/1742-5468/2011/03/P03026, URL http://stacks.iop.org/1742-5468/2011/i=03/a=P03026? key=crossref.24db6a770fefe7e8cdec02cabe975716.

[77] L.-D. Zhang, L.-B. Fu, Mean-field Berry phase of an interacting spin-1/2 system, Europhys. Lett. 93 (3) (2011) 30001, http://dx.doi.org/10.1209/ 0295-5075/93/30001, URL http://stacks.iop.org/0295-5075/93/i=3/a=30001?key=crossref.adc6d3dceae77ed1e3d5049ad649ab72.

[78] X.-X. Zhang, A.-p. Zhang, F.-l. Li, Detecting the multi-spin interaction of an XY spin chain by the geometric phase of a coupled qubit, Phys. Lett. A 376 (30-31) (2012) 2090-2095, http://dx.doi.org/10.1016/j.physleta.2012.05.018, URL https://linkinghub.elsevier.com/retrieve/ pii/S0375960112005634.

[79] Z.-G. Yuan, P. Zhang, S.-S. Li, J. Jing, L.-B. Kong, Scaling of the Berry phase close to the excited-state quantum phase transition in the Lipkin model, Phys. Rev. A 85 (4) (2012) 044102, http://dx.doi.org/10.1103/PhysRevA.85.044102, URL https://link.aps.org/doi/10.1103/PhysRevA.85. 044102.

[80] R. Requist, Hamiltonian formulation of nonequilibrium quantum dynamics: Geometric structure of the Bogoliubov-Born-Green-Kirkwood-Yvon hierarchy, Phys. Rev. A 86 (2) (2012) 022117, http://dx.doi.org/10.1103/PhysRevA.86.022117, URL https://link.aps.org/doi/10.1103/PhysRevA. 86.022117. 
[81] C.-J. Shan, Berry phase and quantum phase transition in spin chain system with three-site interaction, Wuli Xuebao/Acta Phys. Sin. 61 (22) (2012).

[82] M. Tomka, A. Polkovnikov, V. Gritsev, Geometric phase contribution to quantum nonequilibrium many-body dynamics, Phys. Rev. Lett. 108 (8) (2012) 080404, http://dx.doi.org/10.1103/PhysRevLett.108.080404, URL https://link.aps.org/doi/10.1103/PhysRevLett.108.080404.

[83] J. Lian, J.Q. Liang, G. Chen, Geometric phase in the Kitaev honeycomb model and scaling behaviour at critical points, Eur. Phys. J. B 85 (6) (2012) 207, http://dx.doi.org/10.1140/epjb/e2012-20901-1, URL http://www.springerlink.com/index/10.1140/epjb/e2012-20901-1.

[84] Y.-Q. Ma, Z.-X. Yu, D.-S. Wang, B.-H. Xie, X.-G. Li, Momentum space Z 2 number, quantized Berry phase and the quantum phase transitions in spin chain systems, Europhys. Lett. 100 (6) (2012) 60001, http://dx.doi.org/10.1209/0295-5075/100/60001, URL http://stacks.iop.org/02955075/100/i=6/a=60001?key=crossref.d70e83756fa 184dcd4152d727b4d34ea.

[85] Y.-Q. Ma, S.-J. Gu, S. Chen, H. Fan, W.-M. Liu, The Euler number of Bloch states manifold and the quantum phases in gapped fermionic systems, EPL (Europhys. Lett. 103 (1) (2013) 10008, http://dx.doi.org/10.1209/0295-5075/103/10008, URL http://stacks.iop.org/0295-5075/103/ $\mathrm{i}=1 / \mathrm{a}=10008$ ? $\mathrm{key}=$ crossref.dff62933877e7a844bb6359cf0bc8f46.

[86] X. Zhang, Z. Song, Geometric phase and phase diagram for a non-Hermitian quantum XY model, Phys. Rev. A 88 (4) (2013) 042108, http://dx.doi.org/10.1103/PhysRevA.88.042108, URL https://link.aps.org/doi/10.1103/PhysRevA.88.042108.

[87] A.-p. Zhang, F.-l. Li, Geometric phase of a central qubit coupled to a spin chain in a thermal equilibrium state, Phys. Lett. A 377 (7) (2013) 528-533, http://dx.doi.org/10.1016/j.physleta.2012.12.028, URL https://linkinghub.elsevier.com/retrieve/pii/S0375960112013114.

[88] S.-D. Liang, G.-Y. Huang, Topological invariance and global Berry phase in non-Hermitian systems, Phys. Rev. A 87 (1) (2013) 012118 , http://dx.doi.org/10.1103/PhysRevA.87.012118, URL https://link.aps.org/doi/10.1103/PhysRevA.87.012118.

[89] V. Azimi Mousolou, C.M. Canali, E. Sjöqvist, Unifying geometric entanglement and geometric phase in a quantum phase transition, Phys. Rev. A 88 (1) (2013) 012310, http://dx.doi.org/10.1103/PhysRevA.88.012310, URL https://link.aps.org/doi/10.1103/PhysRevA.88.012310.

[90] R. Jafari, Quantum renormalization group approach to geometric phases in spin chains, Phys. Lett. A 377 (45-48) (2013) 3279-3282, http://dx.doi.org/10.1016/j.physleta.2013.10.034, URL https://linkinghub.elsevier.com/retrieve/pii/S0375960113009870.

[91] S.-C. Li, H.-L. Liu, X.-Y. Zhao, Quantum phase transition and geometric phase in a coupled cavity-BEC system, Eur. Phys. J. D 67 (12) (2013) 250, http://dx.doi.org/10.1140/epjd/e2013-40357-1, URL http://link.springer.com/10.1140/epjd/e2013-40357-1.

[92] A.-P. Zhang, F.-L. Li, Induced modification of the geometric phase of a qubit coupled to an XY spin chain by Dzyaloshinsky-Moriya interaction, Chin. Phys. B 22 (3) (2013) 030308, http://dx.doi.org/10.1088/1674-1056/22/3/030308, URL http://stacks.iop.org/1674-1056/22/i=3/a=030308? key=crossref.68fof6dcb27a876014357904c5aa3f10.

[93] S. Sarkar, Quantum criticality of geometric phase in coupled optical cavity arrays under linear quench, Phys. B Condens. Matter 447 (2014) 42-46, http://dx.doi.org/10.1016/j.physb.2014.04.069, URL https://linkinghub.elsevier.com/retrieve/pii/S0921452614003639.

[94] C.-J. Shan, J.-X. Li, W.-W. Cheng, J.-B. Liu, T.-K. Liu, Scaling of geometric phases close to the topological quantum phase transition in Kitaev's quantum wire model, Laser Phys. Lett. 11 (3) (2014) 035202, http://dx.doi.org/10.1088/1612-2011/11/3/035202, URL http://stacks.iop.org/1612202X/11/i=3/a=035202? key=crossref.62f367e79fafe1cb9fof27100ea6fefa.

[95] J.M. Hickey, S. Genway, J.P. Garrahan, Dynamical phase transitions, time-integrated observables, and geometry of states, Phys. Rev. B 89 (5) (2014) 054301, http://dx.doi.org/10.1103/PhysRevB.89.054301, URL https://link.aps.org/doi/10.1103/PhysRevB.89.054301.

[96] J.M. Lü, X.P. Li, L.C. Wang, Geometric phase and the influence of the Dzyaloshinski-Moriya interaction in the one-dimensional quantum compass model, Modern Phys. Lett. B 29 (25) (2015) 1550146, http://dx.doi.org/10.1142/S0217984915501468, URL http://www.worldscientific.com/doi/ abs/10.1142/S0217984915501468.

[97] L.-L. Zhu, Q. Huang, H.-L. Dai, C. Kong, Y.-1. Feng, C.-J. Shan, Detecting topological phase transition in 1D superconducting systems with next nearest neighbor hopping, Laser Phys. Lett. 12 (1) (2015) 015202, http://dx.doi.org/10.1088/1612-2011/12/1/015202, URL http://stacks.iop.org/ $1612-202 X / 12 / \mathrm{i}=1 / \mathrm{a}=015202$ ? $\mathrm{key}=$ crossref.550364ef834166d5e6453ea3f6b452cc.

[98] Z.-G. Yuan, P. Zhang, Critical behavior of the energy gap and its relation with the Berry phase close to the excited state quantum phase transition in the Lipkin model, Chin. Phys. Lett. 32 (6) (2015) 060301, http://dx.doi.org/10.1088/0256-307X/32/6/060301, URL http://stacks.iop.org/0256307X/32/i=6/a=060301?key=crossref.62805bd0019a439f4de6fadea2e3025f.

[99] L. Li, S. Chen, Characterization of topological phase transitions via topological properties of transition points, Phys. Rev. B 92 (8) (2015) 085118, http://dx.doi.org/10.1103/PhysRevB.92.085118, URL https://link.aps.org/doi/10.1103/PhysRevB.92.085118.

[100] W. Wu, D.-W. Luo, J.-B. Xu, Spin echo and geometric phase of a central spin coupled to a compass spin-chain, J. Stat. Mech. Theory Exp. 2015 (1) (2015) P01025, http://dx.doi.org/10.1088/1742-5468/2015/01/P01025, URL http://stacks.iop.org/1742-5468/2015/i=1/a=P01025?key= crossref.b67267e68236cd0c3d4453c14fad6e14.

[101] L. Li, C. Yang, S. Chen, Winding numbers of phase transition points for one-dimensional topological systems, Europhys. Lett. 112 (1) (2015) 10004, http://dx.doi.org/10.1209/0295-5075/112/10004, URL http://stacks.iop.org/0295-5075/112/i=1/a=10004? key=crossref. 07ec774ff89abaafd3ea7475c91ef52f.

[102] Y.-Q. Ma, Ground-state information geometry and quantum criticality in an inhomogeneous spin model, Chin. Phys. B 24 (9) (2015) 090301, http://dx.doi.org/10.1088/1674-1056/24/9/090301, URL http://stacks.iop.org/1674-1056/24/i=9/a=090301? key=crossref. 17383d899f01d658e7b85a092a6f7045.

[103] L. Yang, Y.-Q. Ma, X.-G. Li, Geometric tensor and the topological characterization of the Bloch band in a two-band lattice model, Phys. B Condens. Matter 456 (2015) 359-364, http://dx.doi.org/10.1016/j.physb.2014.09.022, URL https://linkinghub.elsevier.com/retrieve/pii/ S0921452614007509.

[104] A. Zvyagin, Dynamical quantum phase transitions (Review Article), Low Temp. Phys. 42 (11) (2016) 971-994, http://dx.doi.org/10.1063/1. 4969869, URL http://aip.scitation.org/doi/10.1063/1.4969869.

[105] E.-J. Ye, Z.-D. Hu, W. Wu, Scaling of quantum Fisher information close to the quantum phase transition in the XY spin chain, Phys. B Condens. Matter 502 (2016) 151-154, http://dx.doi.org/10.1016/j.physb.2016.08.046, URL https://linkinghub.elsevier.com/retrieve/pii/ S0921452616303891.

[106] W. Nie, F. Mei, L. Amico, L.C. Kwek, Scaling of geometric phase versus band structure in cluster-Ising models, Phys. Rev. E 96 (2) (2017) 020106, http://dx.doi.org/10.1103/PhysRevE.96.020106, URL https://link.aps.org/doi/10.1103/PhysRevE.96.020106.

[107] T.-S. Zeng, W. Zhu, J.-X. Zhu, D.N. Sheng, Nature of continuous phase transitions in interacting topological insulators, Phys. Rev. B 96 (19) (2017) 195118, http://dx.doi.org/10.1103/PhysRevB.96.195118, URL https://link.aps.org/doi/10.1103/PhysRevB.96.195118.

[108] J. Alvarez-Jimenez, A. Dector, J.D. Vergara, Quantum information metric and Berry curvature from a Lagrangian approach, J. High Energy Phys. 2017 (3) (2017) 44, http://dx.doi.org/10.1007/JHEP03(2017)044, URL http://link.springer.com/10.1007/JHEP03(2017)044.

[109] K. Liu, S. Yi, Geometric phase and quantum phase transition in charge-qubit array, Internat. J. Theoret. Phys. 57 (9) (2018) 2828-2830, http://dx.doi.org/10.1007/s10773-018-3802-7, URL http://link.springer.com/10.1007/s10773-018-3802-7.

[110] A. Carollo, B. Spagnolo, D. Valenti, Uhlmann curvature in dissipative phase transitions, Sci. Rep. 8 (1) (2018) 9852, http://dx.doi.org/10.1038/ s41598-018-27362-9, URL http://www.nature.com/articles/s41598-018-27362-9.

[111] A. Carollo, B. Spagnolo, A.A. Dubkov, D. Valenti, On quantumness in multi-parameter quantum estimation, J. Stat. Mech. Theory Exp. 2019 (9) (2019) 094010, http://dx.doi.org/10.1088/1742-5468/ab3ccb, URL https://iopscience.iop.org/article/10.1088/1742-5468/ab3ccb. 
[112] D.-W. Zhang, Y.-Q. Zhu, Y.X. Zhao, H. Yan, S.-L. Zhu, Topological quantum matter with cold atoms, Adv. Phys. 67 (4) (2018) 253-402, http://dx.doi.org/10.1080/00018732.2019.1594094, URL https://www.tandfonline.com/doi/full/10.1080/00018732.2019.1594094.

[113] A. Carollo, B. Spagnolo, D. Valenti, Symmetric logarithmic derivative of fermionic Gaussian states, Entropy 20 (7) (2018) 485, http://dx.doi org/10.3390/e20070485, URL http://www.mdpi.com/1099-4300/20/7/485.

[114] L. Henriet, Geometrical properties of the ground state manifold in the spin boson model, Phys. Rev. B 97 (19) (2018) 195138, http: //dx.doi.org/10.1103/PhysRevB.97.195138, URL https://link.aps.org/doi/10.1103/PhysRevB.97.195138.

[115] X. Cai, R. Meng, Y. Zhang, L. Wang, Geometry of quantum evolution in a nonequilibrium environment, Europhys. Lett. 125 (3) (2019) 30007, http://dx.doi.org/10.1209/0295-5075/125/30007, URL http://stacks.iop.org/0295-5075/125/i=3/a=30007?key=crossref. 20799424937744 fe706a6706cf834255.

[116] W. Lin-Cheng, Y. Jun-Yan, Y. Xue-Xi, Geometric phases and quantum phase transitions in inhomogeneous XY spin-chains: Effect of the Dzyaloshinski-Moriya interaction, Chin. Phys. B 19 (4) (2010) 040512, http://dx.doi.org/10.1088/1674-1056/19/4/040512, URL http://stacks. iop.org/1674-1056/19/i=4/a=040512?key=crossref.a4695c1ca2961db57ec692db98b946a9.

[117] Y.-Q. Ma, Z.-X. Yu, D.-S. Wang, X.-G. Li, Quantized berry phase in twisted bloch momentum space as a topological order parameter for spin chains, Phys. Lett. A 377 (18) (2013) 1250-1254, http://dx.doi.org/10.1016/j.physleta.2013.03.021, URL https://linkinghub.elsevier.com/retrieve/ pii/S0375960113002922.

[118] L. Leonforte, D. Valenti, B. Spagnolo, A. Carollo, Uhlmann number in translational invariant systems, Sci. Rep. 9 (1) (2019) 9106, http: //dx.doi.org/10.1038/s41598-019-45546-9, URL https://rdcu.be/bHE7n.

[119] L. Leonforte, D. Valenti, B. Spagnolo, A.A. Dubkov, A. Carollo, Haldane model at finite temperature, J. Stat. Mech. Theory Exp. 2019 (9) (2019) 094001, http://dx.doi.org/10.1088/1742-5468/ab33f8, URL https://iopscience.iop.org/article/10.1088/1742-5468/ab33f8.

[120] F. Bascone, L. Leonforte, D. Valenti, B. Spagnolo, A. Carollo, Finite-temperature geometric properties of the Kitaev honeycomb model, Phys. Rev. B 99 (20) (2019) http://dx.doi.org/10.1103/PhysRevB.99.205155, URL https://www2.scopus.com/inward/record.uri?eid=2-s2.0-85067188695\&doi= 10.1103\%2FPhysRevB.99.205155\&partnerID=40\&md5=3b87aa48f78d641e92d826a0ebcabb1e.

[121] F. Bascone, L. Leonforte, D. Valenti, B. Spagnolo, A. Carollo, On critical properties of the Berry curvature in the Kitaev honeycomb model, J. Stat Mech. Theory Exp. 2019 (9) (2019) 094002, http://dx.doi.org/10.1088/1742-5468/ab35e9, URL https://iopscience.iop.org/article/10.1088/17425468/ab35e9.

[122] X.-X. Zhang, A.-P. Zhang, J. Zhang, J.-X. Wang, Geometric phase of spin chain system in the nonequilibrium thermal environments, Modern Phys. Lett. B 27 (11) (2013) 1350078, http://dx.doi.org/10.1142/S0217984913500784, URL http://www.worldscientific.com/doi/abs/10.1142/ S0217984913500784.

[123] T. Prosen, I. Pižorn, Quantum phase transition in a far-from-equilibrium steady state of an XY spin chain, Phys. Rev. Lett. 101 (10) (2008) 105701, http://dx.doi.org/10.1103/PhysRevLett.101.105701, URL https://link.aps.org/doi/10.1103/PhysRevLett.101.105701.

[124] S. Diehl, A. Micheli, A. Kantian, B. Kraus, H.P. Büchler, P. Zoller, Quantum states and phases in driven open quantum systems with cold atoms, Nat. Phys. 4 (11) (2008) 878-883, http://dx.doi.org/10.1038/nphys1073, URL http://www.nature.com/articles/nphys 1073.

[125] E.G. Dalla Torre, E. Demler, T. Giamarchi, E. Altman, Quantum critical states and phase transitions in the presence of non-equilibrium noise, Nat. Phys. 6 (10) (2010) 806-810, http://dx.doi.org/10.1038/nphys1754, URL http://www.nature.com/articles/nphys1754.

[126] S. Diehl, A. Tomadin, A. Micheli, R. Fazio, P. Zoller, Dynamical phase transitions and instabilities in open atomic many-body systems, Phys. Rev. Lett. 105 (1) (2010) 015702, http://dx.doi.org/10.1103/PhysRevLett.105.015702, URL https://link.aps.org/doi/10.1103/PhysRevLett.105.015702.

[127] M. Heyl, A. Polkovnikov, S. Kehrein, Dynamical quantum phase transitions in the transverse-field ising model, Phys. Rev. Lett. 110 (13) (2013) 135704, http://dx.doi.org/10.1103/PhysRevLett.110.135704, URL https://link.aps.org/doi/10.1103/PhysRevLett.110.135704.

[128] A. Le Boité, G. Orso, C. Ciuti, Steady-state phases and tunneling-induced instabilities in the driven dissipative bose-hubbard model, Phys. Rev. Lett. 110 (23) (2013) 233601, http://dx.doi.org/10.1103/PhysRevLett.110.233601, URL https://link.aps.org/doi/10.1103/PhysRevLett.110.233601.

[129] C. Carr, R. Ritter, C.G. Wade, C.S. Adams, K.J. Weatherill, Nonequilibrium phase transition in a dilute rydberg ensemble, Phys. Rev. Lett. 111 (11) (2013) 113901, http://dx.doi.org/10.1103/PhysRevLett.111.113901, URL https://link.aps.org/doi/10.1103/PhysRevLett.111.113901.

[130] S. Ajisaka, F. Barra, B. Žunkovič, Nonequilibrium quantum phase transitions in the XY model: comparison of unitary time evolution and reduced density operator approaches, New J. Phys. 16 (3) (2014) 033028, http://dx.doi.org/10.1088/1367-2630/16/3/033028, URL http: //stacks.iop.org/1367-2630/16/i=3/a=033028?key=crossref.bee87b83616e54f615f3e20f1b2d3684.

[131] M. Marcuzzi, E. Levi, S. Diehl, J.P. Garrahan, I. Lesanovsky, Universal nonequilibrium properties of dissipative rydberg gases, Phys. Rev. Lett. 113 (21) (2014) 210401, http://dx.doi.org/10.1103/PhysRevLett.113.210401, URL https://link.aps.org/doi/10.1103/PhysRevLett.113.210401.

[132] S. Vajna, B. Dóra, Topological classification of dynamical phase transitions, Phys. Rev. B 91 (15) (2015) 155127, http://dx.doi.org/10.1103/ PhysRevB.91.155127, URL https://link.aps.org/doi/10.1103/PhysRevB.91.155127.

[133] G. Dagvadorj, J.M. Fellows, S. Matyjaśkiewicz, F.M. Marchetti, I. Carusotto, M.H. Szymańska, Nonequilibrium phase transition in a twodimensional driven open quantum system, Phys. Rev. X 5 (4) (2015) 041028, http://dx.doi.org/10.1103/PhysRevX.5.041028, URL https: //link.aps.org/doi/10.1103/PhysRevX.5.041028.

[134] H. Weimer, Variational principle for steady states of dissipative quantum many-body systems, Phys. Rev. Lett. 114 (4) (2015) 040402 http://dx.doi.org/10.1103/PhysRevLett.114.040402, URL https://link.aps.org/doi/10.1103/PhysRevLett.114.040402.

[135] K. Macieszczak, M. Guţă, I. Lesanovsky, J.P. Garrahan, Towards a theory of metastability in open quantum dynamics, Phys. Rev. Lett. 116 (24) (2016) 240404, http://dx.doi.org/10.1103/PhysRevLett.116.240404, URL https://link.aps.org/doi/10.1103/PhysRevLett.116.240404.

[136] J. Jin, A. Biella, O. Viyuela, L. Mazza, J. Keeling, R. Fazio, D. Rossini, Cluster mean-field approach to the steady-state phase diagram of dissipative spin systems, Phys. Rev. X 6 (3) (2016) 031011, http://dx.doi.org/10.1103/PhysRevX.6.031011, URL https://link.aps.org/doi/10.1103/PhysRevX 6.031011.

[137] D.C. Rose, K. Macieszczak, I. Lesanovsky, J.P. Garrahan, Metastability in an open quantum Ising model, Phys. Rev. E 94 (5) (2016) 052132, http://dx.doi.org/10.1103/PhysRevE.94.052132, URL https://link.aps.org/doi/10.1103/PhysRevE.94.052132.

[138] N. Bartolo, F. Minganti, W. Casteels, C. Ciuti, Exact steady state of a Kerr resonator with one- and two-photon driving and dissipation: Controllable wigner-function multimodality and dissipative phase transitions, Phys. Rev. A 94 (3) (2016) 033841, http://dx.doi.org/10.1103 PhysRevA.94.033841, URL https://link.aps.org/doi/10.1103/PhysRevA.94.033841.

[139] M.F. Maghrebi, A.V. Gorshkov, Nonequilibrium many-body steady states via Keldysh formalism, Phys. Rev. B 93 (1) (2016) 014307, http: //dx.doi.org/10.1103/PhysRevB.93.014307, URL https://link.aps.org/doi/10.1103/PhysRevB.93.014307.

[140] L.M. Sieberer, M. Buchhold, S. Diehl, Keldysh field theory for driven open quantum systems, Rep. Progr. Phys. 79 (9) (2016) 096001, http://dx.doi.org/10.1088/0034-4885/79/9/096001, URL http://stacks.iop.org/0034-4885/79/i=9/a=096001?key=crossref f0f9fd891a0bcd6c69ea234e89466f3a.

[141] S. Roy, R. Moessner, A. Das, Locating topological phase transitions using nonequilibrium signatures in local bulk observables, Phys. Rev. B 95 (4) (2017) 041105, http://dx.doi.org/10.1103/PhysRevB.95.041105, URL https://link.aps.org/doi/10.1103/PhysRevB.95.041105.

[142] J.M. Fink, A. Dombi, A. Vukics, A. Wallraff, P. Domokos, Observation of the photon-blockade breakdown phase transition, Phys. Rev. X 7 (1) (2017) 011012, http://dx.doi.org/10.1103/PhysRevX.7.011012.

[143] M. Fitzpatrick, N.M. Sundaresan, A.C.Y.C. Li, J. Koch, A.A. Houck, Observation of a dissipative phase transition in a one-dimensional circuit QED lattice, Phys. Rev. X 7 (1) (2017) 011016, http://dx.doi.org/10.1103/PhysRevX.7.011016, URL https://link.aps.org/doi/10.1103/PhysRevX.7.011016. 
[144] R. Rota, F. Storme, N. Bartolo, R. Fazio, C. Ciuti, Critical behavior of dissipative two-dimensional spin lattices, Phys. Rev. B 95 (13) (2017) 134431, http://dx.doi.org/10.1103/PhysRevB.95.134431, URL http://link.aps.org/doi/10.1103/PhysRevB.95.134431.

[145] V.R. Overbeck, M.F. Maghrebi, A.V. Gorshkov, H. Weimer, Multicritical behavior in dissipative Ising models, Phys. Rev. A 95 (4) (2017) 042133 , http://dx.doi.org/10.1103/PhysRevA.95.042133, URL http://link.aps.org/doi/10.1103/PhysRevA.95.042133.

[146] M. Foss-Feig, J.T. Young, V.V. Albert, A.V. Gorshkov, M.F. Maghrebi, Solvable family of driven-dissipative many-body systems, Phys. Rev. Lett. 119 (19) (2017) 190402, http://dx.doi.org/10.1103/PhysRevLett.119.190402, URL https://link.aps.org/doi/10.1103/PhysRevLett.119.190402.

[147] J. Jin, A. Biella, O. Viyuela, C. Ciuti, R. Fazio, D. Rossini, Phase diagram of the dissipative quantum Ising model on a square lattice, Phys. Rev. B 98 (24) (2018) 241108, http://dx.doi.org/10.1103/PhysRevB.98.241108, URL https://link.aps.org/doi/10.1103/PhysRevB.98.241108.

[148] R. Rota, F. Minganti, A. Biella, C. Ciuti, Dynamical properties of dissipative XYZ Heisenberg lattices, New J. Phys. 20 (4) (2018) 045003, http://dx. doi.org/10.1088/1367-2630/aab703, URL http://stacks.iop.org/1367-2630/20/i=4/a=045003?key=crossref.803698b626a12881ce8276ba28cc199e.

[149] F. Minganti, A. Biella, N. Bartolo, C. Ciuti, Spectral theory of Liouvillians for dissipative phase transitions, Phys. Rev. A 98 (4) (2018) 042118, http://dx.doi.org/10.1103/PhysRevA.98.042118, URL https://link.aps.org/doi/10.1103/PhysRevA.98.042118.

[150] F. Vicentini, F. Minganti, R. Rota, G. Orso, C. Ciuti, Critical slowing down in driven-dissipative Bose-Hubbard lattices, Phys. Rev. A 97 (1) (2018) 013853, http://dx.doi.org/10.1103/PhysRevA.97.013853, URL https://link.aps.org/doi/10.1103/PhysRevA.97.013853.

[151] A. Nagy, V. Savona, Driven-dissipative quantum Monte Carlo method for open quantum systems, Phys. Rev. A 97 (5) (2018) 052129, http://dx.doi.org/10.1103/PhysRevA.97.052129, URL https://link.aps.org/doi/10.1103/PhysRevA.97.052129.

[152] W. Casteels, R.M. Wilson, M. Wouters, Gutzwiller Monte Carlo approach for a critical dissipative spin model, Phys. Rev. A 97 (6) (2018) 062107, http://dx.doi.org/10.1103/PhysRevA.97.062107, URL https://link.aps.org/doi/10.1103/PhysRevA.97.062107.

[153] R. Rota, F. Minganti, C. Ciuti, V. Savona, Quantum critical regime in a quadratically driven nonlinear photonic lattice, Phys. Rev. Lett. 122 (11) (2019) 110405, http://dx.doi.org/10.1103/PhysRevLett.122.110405, URL https://link.aps.org/doi/10.1103/PhysRevLett.122.110405.

[154] R. Alicki, K. Lendi, Quantum dynamical semigroups and applications, Springer-Verlag, 2007, p. 128.

[155] H.-P. Breuer, F. Petruccione, The Theory of Open Quantum Systems, Oxford University Press, 2007, p. 625, http://dx.doi.org/10.1093/acprof: oso/9780199213900.001.0001.

[156] A. Uhlmann, Parallel transport and "quantum holonomy" along density operators, Rep. Math. Phys. 24 (2) (1986) 229-240, http://dx.doi.org/ 10.1016/0034-4877(86)90055-8, URL http://linkinghub.elsevier.com/retrieve/pii/0034487786900558.

[157] S.L. Braunstein, C.M. Caves, Statistical distance and the geometry of quantum states, Phys. Rev. Lett. 72 (22) (1994) 3439-3443, http: //dx.doi.org/10.1103/PhysRevLett.72.3439, URL https://link.aps.org/doi/10.1103/PhysRevLett.72.3439.

[158] P. Zanardi, N. Paunković, Ground state overlap and quantum phase transitions, Phys. Rev. E 74 (3) (2006) 031123, http://dx.doi.org/10.1103/ PhysRevE.74.031123, URL https://link.aps.org/doi/10.1103/PhysRevE.74.031123.

[159] P. Zanardi, P. Giorda, M. Cozzini, Information-theoretic differential geometry of quantum phase transitions, Phys. Rev. Lett. 99 (10) (2007) 100603, http://dx.doi.org/10.1103/PhysRevLett.99.100603, URL https://link.aps.org/doi/10.1103/PhysRevLett.99.100603.

[160] S.-J.S.-J. Gu, Fidelity approach to quantum phase transitions, Internat. J. Modern Phys. B 24 (23) (2010) 4371-4458, http://dx.doi.org/10.1142/ S0217979210056335, URL http://www.worldscientific.com/doi/abs/10.1142/S0217979210056335.

[161] A. Dey, S. Mahapatra, P. Roy, T. Sarkar, Information geometry and quantum phase transitions in the Dicke model, Phys. Rev. E 86 (3) (2012) 031137, http://dx.doi.org/10.1103/PhysRevE.86.031137, URL https://link.aps.org/doi/10.1103/PhysRevE.86.031137.

[162] H. Janyszek, Riemannian geometry and stability of thermodynamical equilibrium systems, J. Phys. A: Math. Gen. 23 (4) (1990) 477-490, http://dx.doi.org/10.1088/0305-4470/23/4/017, URL http://stacks.iop.org/0305-4470/23/i=4/a=017?key=crossref. d0b7805ad3b73006eb6f93924e228de8.

[163] G. Ruppeiner, Riemannian geometry in thermodynamic fluctuation theory, Rev. Modern Phys. 67 (3) (1995) 605-659, http://dx.doi.org/10. 1103/RevModPhys.67.605, URL https://link.aps.org/doi/10.1103/RevModPhys.67.605.

[164] H.T. Quan, F.M. Cucchietti, Quantum fidelity and thermal phase transitions, Phys. Rev. E 79 (3) (2009) 031101, http://dx.doi.org/10.1103/ PhysRevE.79.031101, URL https://link.aps.org/doi/10.1103/PhysRevE.79.031101.

[165] P. Zanardi, L. Campos Venuti, P. Giorda, Bures metric over thermal state manifolds and quantum criticality, Phys. Rev. A 76 (6) (2007) 062318, http://dx.doi.org/10.1103/PhysRevA.76.062318, URL https://link.aps.org/doi/10.1103/PhysRevA.76.062318.

[166] M. Kolodrubetz, V. Gritsev, A. Polkovnikov, Classifying and measuring geometry of a quantum ground state manifold, Phys. Rev. B 88 (6) (2013) 064304, http://dx.doi.org/10.1103/PhysRevB.88.064304, URL https://link.aps.org/doi/10.1103/PhysRevB.88.064304.

[167] S. Yang, S.-J. Gu, C.-P. Sun, H.-Q. Lin, Fidelity susceptibility and long-range correlation in the kitaev honeycomb model, Phys. Rev. A 78 (1) (2008) 012304, http://dx.doi.org/10.1103/PhysRevA.78.012304, URL https://link.aps.org/doi/10.1103/PhysRevA.78.012304.

[168] E. Sjöqvist, A.K. Pati, A. Ekert, J.S. Anandan, M. Ericsson, D.K.L. Oi, V. Vedral, Geometric phases for mixed states in interferometry, Phys. Rev. Lett. 85 (14) (2000) 2845-2849, http://dx.doi.org/10.1103/PhysRevLett.85.2845, URL https://link.aps.org/doi/10.1103/PhysRevLett.85.2845.

[169] D.M. Tong, E. Sjöqvist, L.C. Kwek, C.H. Oh, Kinematic approach to the mixed state geometric phase in nonunitary evolution, Phys. Rev. Lett. 93 (8) (2004) 080405, http://dx.doi.org/10.1103/PhysRevLett.93.080405, URL https://link.aps.org/doi/10.1103/PhysRevLett.93.080405.

[170] S. Chaturvedi, E. Ercolessi, G. Marmo, G. Morandi, N. Mukunda, R. Simon, Geometric phase for mixed states: a differential geometric approach, Eur. Phys. J. C 35 (3) (2004) 413-423, http://dx.doi.org/10.1140/epjc/s2004-01814-5, URL https://link.springer.com/article/10.1140/epjc/s200401814-5.

[171] K.-P. Marzlin, S. Ghose, B.C. Sanders, Geometric phase distributions for open quantum systems, Phys. Rev. Lett. 93 (26) (2004) 260402, http://dx.doi.org/10.1103/PhysRevLett.93.260402, URL http://link.aps.org/doi/10.1103/PhysRevLett.93.260402.

[172] A. Carollo, The quantum trajectory approach to geometric phase for open systems, Modern Phys. Lett. A 20 (22) (2005) 1635-1654, http://dx.doi.org/10.1142/S0217732305017718, URL http://www.worldscientific.com/doi/abs/10.1142/S0217732305017718.

[173] N. Burić, M. Radonjić, Uniquely defined geometric phase of an open system, Phys. Rev. A 80 (1) (2009) 014101, http://dx.doi.org/10.1103/ PhysRevA.80.014101, URL https://link.aps.org/doi/10.1103/PhysRevA.80.014101.

[174] N.a. Sinitsyn, The stochastic pump effect and geometric phases in dissipative and stochastic systems, J. Phys. A Math. Theor. 42 (19) (2009) 193001, http://dx.doi.org/10.1088/1751-8113/42/19/193001, URL http://stacks.iop.org/1751-8121/42/i=19/a=193001?key=crossref. a38e93a5bcb293540ced1ecbed8ffd30.

[175] K. Matsumoto, A geometrical approach to quantum estimation theory, in: Asymptot. Theory Quantum Stat. Inference, World Scientific, 2005, pp. 305-350, http://dx.doi.org/10.1142/9789812563071_0021, URL http://www.worldscientific.com/doi/abs/10.1142/9789812563071_0021.

[176] M. Hayashi, Quantum information geometry and quantum estimation, in: Quantum Inf. Theory Math. Found., Springer, Berlin, Heidelberg, 2017, pp. 253-322, http://dx.doi.org/10.1007/978-3-662-49725-8_6, URL http://link.springer.com/10.1007/978-3-662-49725-8_6.

[177] L. Banchi, P. Giorda, P. Zanardi, Quantum information-geometry of dissipative quantum phase transitions, Phys. Rev. E 89 (2) (2014) 022102, http://dx.doi.org/10.1103/PhysRevE.89.022102, URL https://link.aps.org/doi/10.1103/PhysRevE.89.022102.

[178] U. Marzolino, T. Prosen, Fisher information approach to nonequilibrium phase transitions in a quantum XXZ spin chain with boundary noise, Phys. Rev. B 96 (10) (2017) 104402, http://dx.doi.org/10.1103/PhysRevB.96.104402, URL https://link.aps.org/doi/10.1103/PhysRevB.96.104402.

[179] Z. Huang, D.P. Arovas, Topological indices for open and thermal systems via uhlmann's phase, Phys. Rev. Lett. 113 (7) (2014) 076407, http://dx.doi.org/10.1103/PhysRevLett.113.076407, URL https://link.aps.org/doi/10.1103/PhysRevLett.113.076407. 
[180] O. Viyuela, A. Rivas, M.A. Martin-Delgado, Uhlmann phase as a topological measure for one-dimensional fermion systems, Phys. Rev. Lett. 112 (13) (2014) 130401, http://dx.doi.org/10.1103/PhysRevLett.112.130401, URL https://link.aps.org/doi/10.1103/PhysRevLett.112.130401.

[181] O. Andersson, I. Bengtsson, M. Ericsson, E. Sjöqvist, Geometric phases for mixed states of the Kitaev chain, Phil. Trans. R. Soc. A 374 (2068) (2016) 20150231, http://dx.doi.org/10.1098/rsta.2015.0231, URL http://rsta.royalsocietypublishing.org/lookup/doi/10.1098/rsta.2015.0231.

[182] O. Viyuela, A. Rivas, M.A. Martin-Delgado, Two-dimensional density-matrix topological fermionic phases: topological uhlmann numbers, Phys. Rev. Lett. 113 (7) (2014) 076408, http://dx.doi.org/10.1103/PhysRevLett.113.076408, URL http://link.aps.org/doi/10.1103/PhysRevLett.113. 076408.

[183] J.C. Budich, S. Diehl, Topology of density matrices, Phys. Rev. B 91 (16) (2015) 165140, http://dx.doi.org/10.1103/PhysRevB.91.165140, URL https://link.aps.org/doi/10.1103/PhysRevB.91.165140.

[184] S.N. Kempkes, A. Quelle, C.M. Smith, Universalities of thermodynamic signatures in topological phases, Sci. Rep. 6 (1) (2016) 38530, http://dx.doi.org/10.1038/srep38530, URL http://www.nature.com/articles/srep38530.

[185] B. Mera, C. Vlachou, N. Paunković, V.R. Vieira, Uhlmann connection in fermionic systems undergoing phase transitions, Phys. Rev. Lett. 119 (1) (2017) 015702, http://dx.doi.org/10.1103/PhysRevLett.119.015702, URL http://link.aps.org/doi/10.1103/PhysRevLett.119.015702.

[186] J. Tidström, E. Sjöqvist, Uhlmann's geometric phase in presence of isotropic decoherence, Phys. Rev. A 67 (3) (2003) 032110, http: //dx.doi.org/10.1103/PhysRevA.67.032110, URL https://link.aps.org/doi/10.1103/PhysRevA.67.032110.

[187] J. Åberg, D. Kult, E. Sjöqvist, D.K.L. Oi, Operational approach to the Uhlmann holonomy, Phys. Rev. A 75 (3) (2007) 032106, http://dx.doi.org/ 10.1103/PhysRevA.75.032106, URL https://link.aps.org/doi/10.1103/PhysRevA.75.032106.

[188] O. Viyuela, A. Rivas, S. Gasparinetti, A. Wallraff, S. Filipp, M.A. Martin-Delgado, Observation of topological Uhlmann phases with superconducting qubits, npj Quantum Inf. 4 (1) (2018) 10, http://dx.doi.org/10.1038/s41534-017-0056-9, URL http://www.nature.com/articles/s41534-0170056-9.

[189] J. Zhu, M. Shi, V. Vedral, X. Peng, D. Suter, J. Du, Experimental demonstration of a unified framework for mixed-state geometric phases, Europhys. Lett. 94 (2) (2011) 20007, http://dx.doi.org/10.1209/0295-5075/94/20007, URL http://stacks.iop.org/0295-5075/94/i=2/a=20007?key= crossref.a5d79ff4f3bf3269582f84fe8ac69272.

[190] S. Ragy, M. Jarzyna, R. Demkowicz-Dobrzański, Compatibility in multiparameter quantum metrology, Phys. Rev. A 94 (5) (2016) 052108, http://dx.doi.org/10.1103/PhysRevA.94.052108, URL https://link.aps.org/doi/10.1103/PhysRevA.94.052108.

[191] J. Eisert, T. Prosen, Noise-driven quantum criticality, 2010, http://arxiv.org/abs/1012.5013.

[192] U. Marzolino, T. Prosen, Quantum metrology with nonequilibrium steady states of quantum spin chains, Phys. Rev. A 90 (6) (2014) 062130, http://dx.doi.org/10.1103/PhysRevA.90.062130, URL https://link.aps.org/doi/10.1103/PhysRevA.90.062130.

[193] W.-L. You, Y.-W. Li, S.-J. Gu, Fidelity, dynamic structure factor, and susceptibility in critical phenomena, Phys. Rev. E 76 (2) (2007) 022101 , http://dx.doi.org/10.1103/PhysRevE.76.022101, URL https://link.aps.org/doi/10.1103/PhysRevE.76.022101.

[194] H.-Q. Zhou, J.P. Barjaktarevič, Fidelity and quantum phase transitions, J. Phys. A 41 (41) (2008) 412001, http://dx.doi.org/10.1088/17518113/41/41/412001, URL http://stacks.iop.org/1751-8121/41/i=41/a=412001?key=crossref.b880de02325394802321f25b3f94dde6.

[195] D. Schwandt, F. Alet, S. Capponi, Quantum Monte Carlo simulations of fidelity at magnetic quantum phase transitions, Phys. Rev. Lett. 103 (17) (2009) 170501, http://dx.doi.org/10.1103/PhysRevLett.103.170501, URL https://link.aps.org/doi/10.1103/PhysRevLett.103.170501.

[196] H.T. Quan, Z. Song, X.F. Liu, P. Zanardi, C.P. Sun, Decay of loschmidt echo enhanced by quantum criticality, Phys. Rev. Lett. 96 (14) (2006) 140604, http://dx.doi.org/10.1103/PhysRevLett.96.140604, URL https://link.aps.org/doi/10.1103/PhysRevLett.96.140604.

[197] P.W. Anderson, Infrared catastrophe in fermi gases with local scattering potentials, Phys. Rev. Lett. 18 (24) (1967) 1049-1051, http: //dx.doi.org/10.1103/PhysRevLett.18.1049, URL https://link.aps.org/doi/10.1103/PhysRevLett.18.1049.

[198] P. Zanardi, M. Cozzini, P. Giorda, Ground state fidelity and quantum phase transitions in free Fermi systems, J. Stat. Mech. Theory Exp. 2007 (02) (2007) L02002, http://dx.doi.org/10.1088/1742-5468/2007/02/L02002, URL http://stacks.iop.org/1742-5468/2007/i=02/a=L02002?key= crossref.afaa941b230791afe9af65fb0d5ab3a3.

[199] M. Cozzini, P. Giorda, P. Zanardi, Quantum phase transitions and quantum fidelity in free fermion graphs, Phys. Rev. B 75 (1) (2007) 014439 http://dx.doi.org/10.1103/PhysRevB.75.014439, URL https://link.aps.org/doi/10.1103/PhysRevB.75.014439.

[200] T. Liu, Y.-Y. Zhang, Q.-H. Chen, K.-L. Wang, Large- N scaling behavior of the ground-state energy, fidelity, and the order parameter in the Dicke model, Phys. Rev. A 80 (2) (2009) 023810, http://dx.doi.org/10.1103/PhysRevA.80.023810, URL https://link.aps.org/doi/10.1103/PhysRevA. 80.023810 .

[201] M. Cozzini, R. Ionicioiu, P. Zanardi, Quantum fidelity and quantum phase transitions in matrix product states, Phys. Rev. B 76 (10) (2007) 104420, http://dx.doi.org/10.1103/PhysRevB.76.104420, URL https://link.aps.org/doi/10.1103/PhysRevB.76.104420.

[202] P. Buonsante, A. Vezzani, Ground-state fidelity and bipartite entanglement in the bose-hubbard model, Phys. Rev. Lett. 98 (11) (2007) 110601, http://dx.doi.org/10.1103/PhysRevLett.98.110601, URL https://link.aps.org/doi/10.1103/PhysRevLett.98.110601.

[203] M. Łạcki, B. Damski, J. Zakrzewski, Numerical studies of ground-state fidelity of the Bose-Hubbard model, Phys. Rev. A 89 (3) (2014) 033625, http://dx.doi.org/10.1103/PhysRevA.89.033625, URL https://link.aps.org/doi/10.1103/PhysRevA.89.033625.

[204] Q. Luo, S. Hu, B. Xi, J. Zhao, X. Wang, Ground-state phase diagram of an anisotropic spin-1/2 model on the triangular lattice, Phys. Rev. B 95 (16) (2017) 165110, http://dx.doi.org/10.1103/PhysRevB.95.165110, URL http://link.aps.org/doi/10.1103/PhysRevB.95.165110.

[205] C. Hickey, L. Cincio, Z. Papić, A. Paramekanti, Emergence of chiral spin liquids via quantum melting of noncoplanar magnetic orders, Phys Rev. B 96 (11) (2017) 115115, http://dx.doi.org/10.1103/PhysRevB.96.115115, URL https://link.aps.org/doi/10.1103/PhysRevB.96.115115.

[206] L. Wang, Y.-H. Liu, J. Imriška, P.N. Ma, M. Troyer, Fidelity susceptibility made simple: A unified quantum monte carlo approach, Phys. Rev. X 5 (3) (2015) 031007, http://dx.doi.org/10.1103/PhysRevX.5.031007, URL https://link.aps.org/doi/10.1103/PhysRevX.5.031007.

[207] M. Weber, M. Hohenadler, Two-dimensional Holstein-Hubbard model: Critical temperature, Ising universality, and bipolaron liquid, Phys. Rev. B 98 (8) (2018) 085405, http://dx.doi.org/10.1103/PhysRevB.98.085405, URL https://link.aps.org/doi/10.1103/PhysRevB.98.085405.

[208] J.-H. Zhao, H.-L. Wang, B. Li, H.-Q. Zhou, Spontaneous symmetry breaking and bifurcations in ground-state fidelity for quantum lattice systems, Phys. Rev. E 82 (6) (2010) 061127, http://dx.doi.org/10.1103/PhysRevE.82.061127, URL https://link.aps.org/doi/10.1103/PhysRevE.82.061127.

[209] Y.H. Su, B.-Q. Hu, S.-H. Li, S.Y. Cho, Quantum fidelity for degenerate ground states in quantum phase transitions, Phys. Rev. E 88 (3) (2013) 032110, http://dx.doi.org/10.1103/PhysRevE.88.032110, URL https://link.aps.org/doi/10.1103/PhysRevE.88.032110.

[210] A. Agarwala, G.K. Gupta, V.B. Shenoy, S. Bhattacharjee, Statistics-tuned phases of pseudofermions in one dimension, Phys. Rev. B 99 (16) (2019) 165125, http://dx.doi.org/10.1103/PhysRevB.99.165125, URL https://link.aps.org/doi/10.1103/PhysRevB.99.165125.

[211] G. Giudici, A. Angelone, G. Magnifico, Z. Zeng, G. Giudice, T. Mendes-Santos, M. Dalmonte, Diagnosing Potts criticality and two-stage melting in one-dimensional hard-core boson models, Phys. Rev. B 99 (9) (2019) 094434, http://dx.doi.org/10.1103/PhysRevB.99.094434, URL https://link.aps.org/doi/10.1103/PhysRevB.99.094434.

[212] D. Rossini, E. Vicari, Ground-state fidelity at first-order quantum transitions, Phys. Rev. E 98 (6) (2018) 062137, http://dx.doi.org/10.1103/ PhysRevE.98.062137, URL https://link.aps.org/doi/10.1103/PhysRevE.98.062137.

[213] S. Chen, L. Wang, Y. Hao, Y. Wang, Intrinsic relation between ground-state fidelity and the characterization of a quantum phase transition, Phys. Rev. A 77 (3) (2008) 032111, http://dx.doi.org/10.1103/PhysRevA.77.032111, URL https://link.aps.org/doi/10.1103/PhysRevA.77.032111.

[214] P. Zanardi, H.T. Quan, X. Wang, C.P. Sun, Mixed-state fidelity and quantum criticality at finite temperature, Phys. Rev. A 75 (3) (2007) 032109 , http://dx.doi.org/10.1103/PhysRevA.75.032109, URL https://link.aps.org/doi/10.1103/PhysRevA.75.032109. 
[215] C. De Grandi, V. Gritsev, A. Polkovnikov, Quench dynamics near a quantum critical point, Phys. Rev. B 81 (1) (2010) 012303, http: //dx.doi.org/10.1103/PhysRevB.81.012303, URL https://link.aps.org/doi/10.1103/PhysRevB.81.012303.

[216] A. Polkovnikov, K. Sengupta, A. Silva, M. Vengalattore, Colloquium : Nonequilibrium dynamics of closed interacting quantum systems, Rev. Modern Phys. 83 (3) (2011) 863-883, http://dx.doi.org/10.1103/RevModPhys.83.863, URL https://link.aps.org/doi/10.1103/RevModPhys.83.863.

[217] J. Hannukainen, J. Larson, Dissipation driven quantum phase transitions and symmetry breaking, Phys. Rev. A 98 (4) (2017) 042113, http://dx.doi.org/10.1103/PhysRevA.98.042113, URL https://link.aps.org/doi/10.1103/PhysRevA.98.042113.

[218] M.-F. Yang, Ground-state fidelity in one-dimensional gapless models, Phys. Rev. B 76 (18) (2007) 180403, http://dx.doi.org/10.1103/PhysRevB. 76.180403, URL https://link.aps.org/doi/10.1103/PhysRevB.76.180403.

[219] J.O. Fjærestad, Ground state fidelity of Luttinger liquids: a wavefunctional approach, J. Stat. Mech. Theory Exp. 2008 (07) (2008) P07011, http://dx.doi.org/10.1088/1742-5468/2008/07/P07011, URL http://stacks.iop.org/1742-5468/2008/i=07/a=P07011?key=crossref f3200e9f0e89785fb222b51041921820.

[220] B. Wang, M. Feng, Z.-Q. Chen, Berezinskii-Kosterlitz-Thouless transition uncovered by the fidelity susceptibility in the XXZ model, Phys. Rev. A 81 (6) (2010) 064301, http://dx.doi.org/10.1103/PhysRevA.81.064301, URL https://link.aps.org/doi/10.1103/PhysRevA.81.064301.

[221] H.-L. Wang, A.-M. Chen, B. Li, H.-Q. Zhou, Ground-state fidelity and Kosterlitz-Thouless phase transition for the spin-1/2 Heisenberg chain with next-to-the-nearest-neighbor interaction, J. Phys. A: Math. Theor. 45 (1) (2012) 015306, http://dx.doi.org/10.1088/1751-8113/45/1/015306, URL http://stacks.iop.org/1751-8121/45/i=1/a=015306?key=crossref.5a3d48ccd5d45dda6c9ffa 18729037a7.

[222] G. Sun, A. Kolezhuk, T. Vekua, Fidelity at Berezinskii-Kosterlitz-Thouless quantum phase transitions, Phys. Rev. B 91 (1) (2015) 014418, http://dx.doi.org/10.1103/PhysRevB.91.014418, URL https://link.aps.org/doi/10.1103/PhysRevB.91.014418.

[223] D.F. Abasto, A. Hamma, P. Zanardi, Fidelity analysis of topological quantum phase transitions, Phys. Rev. A 78 (1) (2008) 010301 , http: //dx.doi.org/10.1103/PhysRevA.78.010301, URL https://link.aps.org/doi/10.1103/PhysRevA.78.010301.

[224] J.-H. Zhao, H.-Q. Zhou, Singularities in ground-state fidelity and quantum phase transitions for the Kitaev model, Phys. Rev. B 80 (1) (2009) 014403, http://dx.doi.org/10.1103/PhysRevB.80.014403, URL https://link.aps.org/doi/10.1103/PhysRevB.80.014403.

[225] S. Garnerone, D. Abasto, S. Haas, P. Zanardi, Fidelity in topological quantum phases of matter, Phys. Rev. A 79 (3) (2009) 032302, http://dx.doi.org/10.1103/PhysRevA.79.032302, URL https://link.aps.org/doi/10.1103/PhysRevA.79.032302.

[226] M. Rigol, B.S. Shastry, S. Haas, Fidelity and superconductivity in two-dimensional t-J models, Phys. Rev. B 80 (9) (2009) 094529, http: //dx.doi.org/10.1103/PhysRevB.80.094529, URL https://link.aps.org/doi/10.1103/PhysRevB.80.094529.

[227] C.J. Jia, B. Moritz, C.-C. Chen, B.S. Shastry, T.P. Devereaux, Fidelity study of the superconducting phase diagram in the two-dimensional single-band Hubbard model, Phys. Rev. B 84 (12) (2011) 125113, http://dx.doi.org/10.1103/PhysRevB.84.125113, URL https://link.aps.org/doi/ 10.1103/PhysRevB.84.125113.

[228] A.F. Albuquerque, F. Alet, C. Sire, S. Capponi, Quantum critical scaling of fidelity susceptibility, Phys. Rev. B 81 (6) (2010) 064418, http://dx.doi.org/10.1103/PhysRevB.81.064418, URL https://link.aps.org/doi/10.1103/PhysRevB.81.064418.

[229] M.M. Rams, B. Damski, Quantum fidelity in the thermodynamic limit, Phys. Rev. Lett. 106 (5) (2011) 055701, http://dx.doi.org/10.1103/ PhysRevLett.106.055701, URL https://link.aps.org/doi/10.1103/PhysRevLett.106.055701.

[230] P. Zanardi, M.G.A. Paris, L. Campos Venuti, Quantum criticality as a resource for quantum estimation, Phys. Rev. A 78 (4) (2008) 042105, http://dx.doi.org/10.1103/PhysRevA.78.042105, URL https://link.aps.org/doi/10.1103/PhysRevA.78.042105.

[231] J. Zhang, X. Peng, N. Rajendran, D. Suter, Detection of quantum critical points by a probe qubit, Phys. Rev. Lett. 100 (10) (2008) http:// dx.doi.org/10.1103/PhysRevLett.100.100501, URL https://www.scopus.com/inward/record.uri?eid=2-s2.0-40849106560\&doi=10.1103partnerID= 40\&md5=2aca07340f2f562aad524a1967f7223e

[232] S.-J. Gu, W.C. Yu, Spectral function and fidelity susceptibility in quantum critical phenomena, EPL (Europhys. Lett.) 108 (2) (2014) 20002, http://dx.doi.org/10.1209/0295-5075/108/20002, URL http://stacks.iop.org/0295-5075/108/i=2/a=20002?key=crossref. 3cefa42889fe82c4c7635fae7fa9e020.

[233] D.T. Tran, A. Dauphin, A.G. Grushin, P. Zoller, N. Goldman, Probing topology by "heating": Quantized circular dichroism in ultracold atoms, Sci. Adv. 3 (8) (2017) e1701207, http://dx.doi.org/10.1126/sciadv.1701207, URL http://advances.sciencemag.org/lookup/doi/10.1126/sciadv.1701207.

[234] D.J. Amit, Field Theory, the Renormalization Group, and Critical Phenomena, World Scientific, 1984, p. 543.

[235] G. Herzberg, H.C. Longuet-Higgins, Intersection of potential energy surfaces in polyatomic molecules, Discuss. Faraday Soc. 35 (1963) $77-82$ http://dx.doi.org/10.1039/df9633500077.

[236] A.J. Stone, Spin-orbit coupling and the intersection of potential energy surfaces in polyatomic molecules, Proc. R. Soc. A 351 (1664) (1976) 141-150, http://dx.doi.org/10.1098/rspa.1976.0134, URL http://rspa.royalsocietypublishing.org/cgi/doi/10.1098/rspa.1976.0134.

[237] N. Johansson, E. Sjöqvist, Optimal topological test for degeneracies of real hamiltonians, Phys. Rev. Lett. 92 (6) (2004) 060406, http: //dx.doi.org/10.1103/PhysRevLett.92.060406, URL https://link.aps.org/doi/10.1103/PhysRevLett.92.060406.

[238] N. Johansson, E. Sjöqvist, Searching for degeneracies of real Hamiltonians using homotopy classification of loops in SO(n), Phys. Rev. A 71 (1) (2005) 012106, http://dx.doi.org/10.1103/PhysRevA.71.012106, URL https://link.aps.org/doi/10.1103/PhysRevA.71.012106.

[239] E.H. Lieb, T. Schultz, D. Mattis, Two soluble models of an antiferromagnetic chain, Ann. Phys. (N. Y). 16 (3) (1961) 407-466, http: //dx.doi.org/10.1016/0003-4916(61)90115-4.

[240] S. Katsura, Statistical mechanics of the anisotropic linear heisenberg model, Phys. Rev. 127 (5) (1962) 1508-1518, http://dx.doi.org/10.1103/ PhysRev.127.1508, URL https://link.aps.org/doi/10.1103/PhysRev.127.1508.

[241] P. de Gennes, Collective motions of hydrogen bonds, Solid State Commun. 1 (6) (1963) 132-137, http://dx.doi.org/10.1016/0038-1098(63) 90212-6, URL http://linkinghub.elsevier.com/retrieve/pii/0038109863902126.

[242] R.H. Dicke, Coherence in spontaneous radiation processes, Phys. Rev. 93 (1) (1954) 99-110, http://dx.doi.org/10.1103/PhysRev.93.99, URL https://link.aps.org/doi/10.1103/PhysRev.93.99.

[243] K. Hepp, E.H. Lieb, On the superradiant phase transition for molecules in a quantized radiation field: the dicke maser model, Ann. Phys. (N. Y). 76 (2) (1973) 360-404, http://dx.doi.org/10.1016/0003-4916(73)90039-0, URL http://linkinghub.elsevier.com/retrieve/pii/0003491673900390.

[244] K. Hepp, E.H. Lieb, Equilibrium statistical mechanics of matter interacting with the quantized radiation field, Phys. Rev. A 8 (5) (1973) 2517-2525, http://dx.doi.org/10.1103/PhysRevA.8.2517, URL https://link.aps.org/doi/10.1103/PhysRevA.8.2517.

[245] Y.K. Wang, F.T. Hioe, Phase transition in the dicke model of superradiance, Phys. Rev. A 7 (3) (1973) 831-836, http://dx.doi.org/10.1103/ PhysRevA.7.831, URL https://link.aps.org/doi/10.1103/PhysRevA.7.831.

[246] G.C. Duncan, Effect of antiresonant atom-field interactions on phase transitions in the Dicke model, Phys. Rev. A 9 (1) (1974) 418-421, http://dx.doi.org/10.1103/PhysRevA.9.418, URL https://link.aps.org/doi/10.1103/PhysRevA.9.418.

[247] R. Gilmore, C.M. Bowden, Coupled order-parameter treatment of the Dicke Hamiltonian, Phys. Rev. A 13 (5) (1976) 1898-1907, http: //dx.doi.org/10.1103/PhysRevA.13.1898, URL https://link.aps.org/doi/10.1103/PhysRevA.13.1898.

[248] M. Orszag, Phase transition of a system of two-level atoms, J. Phys. A: Math. Gen. 10 (11) (1977) 1995-2005, http://dx.doi.org/10.1088/03054470/10/11/025, URL http://stacks.iop.org/0305-4470/10/i=11/a=025?key=crossref.14ca5176b21b0f0e45bf9c60cd47d703.

[249] S. Sivasubramanian, A. Widom, Y. Srivastava, Gauge invariant formulations of Dicke -Preparata super-radiant models, Phys. A 301 (1-4) (2001) 241-254, http://dx.doi.org/10.1016/S0378-4371(01)00384-3, URL https://linkinghub.elsevier.com/retrieve/pii/S0378437101003843. 
[250] G. Liberti, R.L. Zaffino, Critical properties of two-level atom systems interacting with a radiation field, Phys. Rev. A 70 (3) (2004) 033808, http://dx.doi.org/10.1103/PhysRevA.70.033808, URL https://link.aps.org/doi/10.1103/PhysRevA.70.033808.

[251] G. Liberti, R.L. Zaffino, Thermodynamic properties of the Dicke model in the strong-coupling regime, Eur. Phys. J. B 44 (4) (2005) 535-541, http://dx.doi.org/10.1140/epjb/e2005-00153-0, URL http://www.springerlink.com/index/10.1140/epjb/e2005-00153-0.

[252] S. Schneider, G.J. Milburn, Entanglement in the steady state of a collective-angular-momentum (Dicke) model, Phys. Rev. A 65 (4) (2002) 042107, http://dx.doi.org/10.1103/PhysRevA.65.042107, URL https://link.aps.org/doi/10.1103/PhysRevA.65.042107.

[253] C. Emary, T. Brandes, Quantum chaos triggered by precursors of a quantum phase transition: the Dicke model, Phys. Rev. Lett. 90 (4) (2003) 044101, http://dx.doi.org/10.1103/PhysRevLett.90.044101, URL https://link.aps.org/doi/10.1103/PhysRevLett.90.044101.

[254] C. Emary, T. Brandes, Chaos and the quantum phase transition in the Dicke model, Phys. Rev. E 67 (6) (2003) 066203, http://dx.doi.org/10. 1103/PhysRevE.67.066203, URL https://link.aps.org/doi/10.1103/PhysRevE.67.066203.

[255] M. Frasca, 1/N-Expansion for the Dicke model and the decoherence program, Ann. Phys. (N. Y). 313 (1) (2004) 26-36, http://dx.doi.org/10. 1016/j.aop.2004.04.005, URL http://linkinghub.elsevier.com/retrieve/pii/S0003491604000685.

[256] X.-W. Hou, B. Hu, Decoherence, entanglement, and chaos in the Dicke model, Phys. Rev. A 69 (4) (2004) 042110, http://dx.doi.org/10.1103/ PhysRevA.69.042110, URL https://link.aps.org/doi/10.1103/PhysRevA.69.042110.

[257] V. Bužek, M. Orszag, M. Roško, Instability and entanglement of the ground state of the Dicke model, Phys. Rev. Lett. 94 (16) (2005) 163601, http://dx.doi.org/10.1103/PhysRevLett.94.163601, URL https://link.aps.org/doi/10.1103/PhysRevLett.94.163601.

[258] T. Brandes, Coherent and collective quantum optical effects in mesoscopic systems, Phys. Rep. 408 (5-6) (2005) 315-474, http://dx.doi.org/ 10.1016/j.physrep.2004.12.002, URL http://linkinghub.elsevier.com/retrieve/pii/S0370157304005496.

[259] N. Lambert, C. Emary, T. Brandes, Entanglement and the phase transition in single-mode superradiance, Phys. Rev. Lett. 92 (7) (2004) 073602, http://dx.doi.org/10.1103/PhysRevLett.92.073602, URL https://link.aps.org/doi/10.1103/PhysRevLett.92.073602.

[260] J. Reslen, L. Quiroga, N.F. Johnson, Direct equivalence between quantum phase transition phenomena in radiation-matter and magnetic systems: Scaling of entanglement, Europhys. Lett. 69 (1) (2005) 8-14, http://dx.doi.org/10.1209/epl/i2004-10313-4, URL http://stacks.iop.org/02955075/69/i=1/a=008?key=crossref.df7af9c3e3dc146bcbce9a6c54aa0f3d.

[261] J. Vidal, S. Dusuel, Finite-size scaling exponents in the Dicke model, Europhys. Lett. 74 (5) (2006) 817-822, http://dx.doi.org/10.1209/epl/i200610041-9, URL http://stacks.iop.org/0295-5075/74/i=5/a=817?key=crossref.21d1dda5940a2437d3781acf6a68eea0.

[262] G. Liberti, R.L. Zaffino, F. Piperno, F. Plastina, Entanglement of a qubit coupled to a resonator in the adiabatic regime, Phys. Rev. A 73 (3) (2006) 032346, http://dx.doi.org/10.1103/PhysRevA.73.032346, URL https://link.aps.org/doi/10.1103/PhysRevA.73.032346.

[263] G. Liberti, F. Plastina, F. Piperno, Scaling behavior of the adiabatic Dicke model, Phys. Rev. A 74 (2) (2006) 022324, http://dx.doi.org/10.1103/ PhysRevA.74.022324, URL https://link.aps.org/doi/10.1103/PhysRevA.74.022324.

[264] B. Simon, A. Dicke, Coupling constant analyticity for the anharmonic oscillator, Ann. Phys. (N. Y). 58 (1) (1970) 76-136, http://dx.doi.org/10. 1016/0003-4916(70)90240-X, URL http://linkinghub.elsevier.com/retrieve/pii/000349167090240X.

[265] G. Fubini, Sulle metriche definite da una forma hermitiana, Atti Istit. Veneto LXIII (2) (1904) 501-511.

[266] Eduard Study, Kürzeste Wege im komplexen Gebiet, Math. Ann. 60 (1905) 321-378.

[267] J.P. Provost, G. Vallee, Riemannian structure on manifolds of quantum states, Comm. Math. Phys. 76 (3) (1980) 289-301, http://dx.doi.org/10. 1007/BF02193559, URL http://link.springer.com/10.1007/BF02193559.

[268] M.B. Hastings, Locality in quantum and markov dynamics on lattices and networks, Phys. Rev. Lett. 93 (14) (2004) 140402, http://dx.doi.org/ 10.1103/PhysRevLett.93.140402, URL https://link.aps.org/doi/10.1103/PhysRevLett.93.140402.

[269] L. Magazzù, D. Valenti, A. Carollo, B. Spagnolo, Multi-state quantum dissipative dynamics in sub-ohmic environment: The strong coupling regime, Entropy 17 (4) (2015) 2341-2354, http://dx.doi.org/10.3390/e17042341, URL http://www.mdpi.com/1099-4300/17/4/2341.

[270] B. Spagnolo, A. Carollo, D. Valenti, Enhancing metastability by dissipation and driving in an asymmetric bistable quantum system, Entropy 20 (4) (2018) 226, http://dx.doi.org/10.3390/e20040226, URL http://www.mdpi.com/1099-4300/20/4/226.

[271] D. Valenti, A. Carollo, B. Spagnolo, Stabilizing effect of driving and dissipation on quantum metastable states, Phys. Rev. A 97 (4) (2018) 042109, http://dx.doi.org/10.1103/PhysRevA.97.042109, URL https://link.aps.org/doi/10.1103/PhysRevA.97.042109.

[272] C. Guarcello, D. Valenti, A. Carollo, B. Spagnolo, Effects of Lévy noise on the dynamics of sine-Gordon solitons in long Josephson junctions, J. Stat. Mech. Theory Exp. 2016 (5) (2016) 054012, http://dx.doi.org/10.1088/1742-5468/2016/05/054012, URL http://stacks.iop.org/17425468/2016/i=5/a=054012?key=crossref.48e7f7f504b019713822c481ca8bac97.

[273] B. Spagnolo, C. Guarcello, L. Magazzù, A. Carollo, D. Persano Adorno, D. Valenti, Nonlinear relaxation phenomena in metastable condensed matter systems, Entropy 19 (1) (2017) 20, http://dx.doi.org/10.3390/e19010020, URL http://www.mdpi.com/1099-4300/19/1/20.

[274] B. Spagnolo, D. Valenti, C. Guarcello, A. Carollo, D. Persano Adorno, S. Spezia, N. Pizzolato, B. Di Paola, Noise-induced effects in nonlinear relaxation of condensed matter systems, Chaos Solitons Fractals 81 (2015) 412-424, http://dx.doi.org/10.1016/j.chaos.2015.07.023.

[275] B. Spagnolo, A. Carollo, D. Valenti, Stabilization by dissipation and stochastic resonant activation in quantum metastable systems, Eur. Phys. J. Spec. Top. 227 (3-4) (2019) 379-420, http://dx.doi.org/10.1140/epjst/e2018-00121-x, URL http://link.springer.com/10.1140/epjst/e2018-00121$\mathrm{X}$.

[276] C. Guarcello, D. Valenti, B. Spagnolo, V. Pierro, G. Filatrella, Anomalous transport effects on switching currents of graphene-based Josephson junctions, Nanotechnology 28 (2017) 134001.

[277] C. Guarcello, D. Valenti, B. Spagnolo, V. Pierro, G. Filatrella, Josephson-based threshold detector for Lèvy-distributed current fluctuations, Phys Rev. Applied 11 (2019) 044078.

[278] J. Dittmann, A. Uhlmann, Connections and metrics respecting purification of quantum states, J. Math. Phys. 40 (7) (1999) 3246-3267, http://dx.doi.org/10.1063/1.532884, URL http://aip.scitation.org/doi/10.1063/1.532884.

[279] A.D. Kiselev, V.V. Kesaev, Interferometric and Uhlmann phases of mixed polarization states, Phys. Rev. A 98 (3) (2018) 033816, http: //dx.doi.org/10.1103/PhysRevA.98.033816, URL https://link.aps.org/doi/10.1103/PhysRevA.98.033816.

[280] A. Uhlmann, On berry phases along mixtures of states, Ann. Phys. 501 (1) (1989) 63-69, http://dx.doi.org/10.1002/andp.19895010108, URL http://doi.wiley.com/10.1002/andp.19895010108.

[281] A. Uhlmann, A gauge field governing parallel transport along mixed states, Lett. Math. Phys. 21 (3) (1991) 229-236, http://dx.doi.org/10.1007/ BF00420373, URL http://link.springer.com/10.1007/BF00420373.

[282] H. Araki, G.A. Raggio, A remark on transition probability, Lett. Math. Phys. 6 (3) (1982) 237-240, http://dx.doi.org/10.1007/BF00403278, URL http://link.springer.com/10.1007/BF00403278.

[283] A. Uhlmann, The metric of bures and the geometric phase, in: G. R., L. J., P. Z. (Eds.), Groups Relat. Top., in: Mathematical Physics Studies, no. 13, Springer, Dordrecht, Dordrecht, 1992, pp. 267-274, http://dx.doi.org/10.1007/978-94-011-2801-8_23, URL http://www.springerlink.com/ index/10.1007/978-94-011-2801-8_23.

[284] C.A. Fuchs, C.M. Caves, Mathematical techniques for quantum communication theory, Open Syst. Inf. Dyn. 3 (3) (1995) 345-356, http: //dx.doi.org/10.1007/BF02228997, URL http://link.springer.com/10.1007/BF02228997.

[285] M.A. Nielsen, I.L. Chuang, Quantum Computation and Quantum Information, Cambridge University Press, Cambridge, 2000.

[286] L. Dabrowski, A. Jadczyk, Quantum statistical holonomy, J. Phys. A: Math. Gen. 22 (15) (1989) 3167-3170, http://dx.doi.org/10.1088/03054470/22/15/032, URL http://stacks.iop.org/0305-4470/22/i=15/a=032?key=crossref.c86a4673fdfbfc06a65fffd8d3aa0120. 
[287] L. Dabrowski, H. Grosse, On quantum holonomy for mixed states, Lett. Math. Phys. 19 (3) (1990) 205-210, http://dx.doi.org/10.1007/ BF01039313, URL http://link.springer.com/10.1007/BF01039313.

[288] H.-J. rgen Sommers, K. Zyczkowski, Bures volume of the set of mixed quantum states, J. Phys. A: Math. Gen. 36 (39) (2003) 10083-10100, http://dx.doi.org/10.1088/0305-4470/36/39/308, URL http://stacks.iop.org/0305-4470/36/i=39/a=308?key=crossref. f55a540ab9f625599586caed4b66bf3a.

[289] D. Šafránek, Discontinuities of the quantum Fisher information and the Bures metric, Phys. Rev. A 95 (5) (2017) 052320, http://dx.doi.org/10. 1103/PhysRevA.95.052320, URL http://link.aps.org/doi/10.1103/PhysRevA.95.052320.

[290] K.M.R. Audenaert, J. Calsamiglia, R. Muñoz-Tapia, E. Bagan, L. Masanes, A. Acin, F. Verstraete, Discriminating states: The quantum chernoff bound, Phys. Rev. Lett. 98 (16) (2007) 160501, http://dx.doi.org/10.1103/PhysRevLett.98.160501, URL https://link.aps.org/doi/10.1103/ PhysRevLett.98.160501.

[291] H. Chernoff, A measure of asymptotic efficiency for tests of a hypothesis based on the sum of observations, Ann. Math. Stat. 23 (4) (1952) 493-507, http://dx.doi.org/10.1214/aoms/1177729330, URL http://www.jstor.org/stable/2236576.

[292] T.M. Cover, J.A. Thomas, Elements of Information Theory, Wiley-Interscience, 2006, p. 748.

[293] C.W. Helstrom, Quantum Detection and Estimation Theory, Academic Press, 1976.

[294] T. Udem, R. Holzwarth, T. Hänsch, Optical frequency metrology, Nature 416 (6877) (2002) 233-237, http://dx.doi.org/10.1038/416233a, URL http://www.nature.com/articles/416233a.

[295] H. Katori, Optical lattice clocks and quantum metrology, Nat. Photonics 5 (4) (2011) 203-210, http://dx.doi.org/10.1038/nphoton.2011.45, URL http://www.nature.com/articles/nphoton.2011.45.

[296] V. Giovannetti, Quantum-enhanced measurements: Beating the standard quantum limit, Science 306 (5700) (2004) 1330-1336, http://dx.doi. org/10.1126/science.1104149, URL http://www.sciencemag.org/cgi/doi/10.1126/science.1104149.

[297] M. Aspachs, G. Adesso, I. Fuentes, Optimal quantum estimation of the unruh-hawking effect, Phys. Rev. Lett. 105 (15) (2010) 151301, http://dx.doi.org/10.1103/PhysRevLett.105.151301, URL https://link.aps.org/doi/10.1103/PhysRevLett.105.151301.

[298] M. Ahmadi, D.E. Bruschi, I. Fuentes, Quantum metrology for relativistic quantum fields, Phys. Rev. D 89 (6) (2014) 065028, http://dx.doi.org/ 10.1103/PhysRevD.89.065028, URL https://link.aps.org/doi/10.1103/PhysRevD.89.065028.

[299] R. Schnabel, N. Mavalvala, D.E. McClelland, P.K. Lam, Quantum metrology for gravitational wave astronomy, Nature Commun. 1 (8) (2010) 110-121, http://dx.doi.org/10.1038/ncomms1122.

[300] J. Aasi, Enhanced sensitivity of the LIGO gravitational wave detector by using squeezed states of light, Nat. Photonics 7 (8) (2013) 613-619, http://dx.doi.org/10.1038/nphoton.2013.177, URL http://www.nature.com/articles/nphoton.2013.177.

[301] L.A. Correa, M. Mehboudi, G. Adesso, A. Sanpera, Individual quantum probes for optimal thermometry, Phys. Rev. Lett. 114 (22) (2015) 220405, http://dx.doi.org/10.1103/PhysRevLett.114.220405, URL https://link.aps.org/doi/10.1103/PhysRevLett.114.220405.

[302] A. De Pasquale, D. Rossini, R. Fazio, V. Giovannetti, Local quantum thermal susceptibility, Nature Commun. 7 (1) (2016) 12782, http: //dx.doi.org/10.1038/ncomms12782, URL http://www.nature.com/articles/ncomms12782.

[303] S. Schmitt, T. Gefen, F.M. Stürner, T. Unden, G. Wolff, C. Müller, J. Scheuer, B. Naydenov, M. Markham, S. Pezzagna, J. Meijer, I. Schwarz, M. Plenio, A. Retzker, L.P. McGuinness, F. Jelezko, Submillihertz magnetic spectroscopy performed with a nanoscale quantum sensor, Science 356 (6340) (2017) 832-837, http://dx.doi.org/10.1126/science.aam5532, URL http://www.sciencemag.org/lookup/doi/10.1126/science.aam5532.

[304] J.M. Boss, K.S. Cujia, J. Zopes, C.L. Degen, Quantum sensing with arbitrary frequency resolution, Science 356 (6340) (2017) 837-840, http://dx.doi.org/10.1126/science.aam7009.

[305] M. Tsang, R. Nair, X.-M. Lu, Quantum theory of superresolution for two incoherent optical point sources, Phys. Rev. X 6 (3) (2016) 031033, http://dx.doi.org/10.1103/PhysRevX.6.031033, URL https://link.aps.org/doi/10.1103/PhysRevX.6.031033.

[306] R. Nair, M. Tsang, Far-field superresolution of thermal electromagnetic sources at the quantum limit, Phys. Rev. Lett. 117 (19) (2016) 190801 , http://dx.doi.org/10.1103/PhysRevLett.117.190801, URL https://link.aps.org/doi/10.1103/PhysRevLett.117.190801.

[307] C. Lupo, S. Pirandola, Ultimate precision bound of quantum and subwavelength imaging, Phys. Rev. Lett. 117 (19) (2016) 190802, http: //dx.doi.org/10.1103/PhysRevLett.117.190802, URL https://link.aps.org/doi/10.1103/PhysRevLett.117.190802.

[308] C.M. Caves, Quantum-mechanical noise in an interferometer, Phys. Rev. D 23 (8) (1981) 1693-1708, http://dx.doi.org/10.1103/PhysRevD.23. 1693.

[309] S.F. Huelga, C. Macchiavello, T. Pellizzari, A.K. Ekert, M.B. Plenio, J.I. Cirac, Improvement of frequency standards with quantum entanglement, Phys. Rev. Lett. 79 (20) (1997) 3865-3868, http://dx.doi.org/10.1103/PhysRevLett.79.3865, URL https://link.aps.org/doi/10.1103/PhysRevLett.79. 3865.

[310] V. Giovannetti, S. Lloyd, L. Maccone, Quantum metrology, Phys. Rev. Lett. 96 (1) (2006) 010401, http://dx.doi.org/10.1103/PhysRevLett.96. 010401, URL https://link.aps.org/doi/10.1103/PhysRevLett.96.010401.

[311] M.G.A. Paris, Quantum estimation for quantum technology, Int. J. Quantum Inf. 07 (supp01) (2009) 125-137, http://dx.doi.org/10.1142/ S0219749909004839, URL http://www.worldscientific.com/doi/abs/10.1142/S0219749909004839.

[312] V. Giovannetti, S. Lloyd, L. Maccone, Advances in quantum metrology, Nat. Photonics 5 (4) (2011) 222-229, http://dx.doi.org/10.1038/nphoton. 2011.35, URL http://www.nature.com/articles/nphoton.2011.35.

[313] G. Tóth, I. Apellaniz, Quantum metrology from a quantum information science perspective, J. Phys. A Math. Theor. 47 (42) (2014) 424006 , http://dx.doi.org/10.1088/1751-8113/47/42/424006, URL https://iopscience.iop.org/article/10.1088/1751-8113/47/42/424006/pdf.

[314] M. Szczykulska, T. Baumgratz, A. Datta, Multi-parameter quantum metrology, Adv. Phys. X 1 (4) (2016) 621-639, http://dx.doi.org/10.1080/ 23746149.2016.1230476, URL https://www.tandfonline.com/doi/full/10.1080/23746149.2016.1230476.

[315] L. Pezzè, A. Smerzi, M.K. Oberthaler, R. Schmied, P. Treutlein, Quantum metrology with nonclassical states of atomic ensembles, Rev. Modern Phys. 90 (3) (2018) 035005, http://dx.doi.org/10.1103/RevModPhys.90.035005, URL https://link.aps.org/doi/10.1103/RevModPhys.90.035005.

[316] R. Nichols, P. Liuzzo-Scorpo, P.A. Knott, G. Adesso, Multiparameter Gaussian quantum metrology, Phys. Rev. A 98 (1) (2018) 012114, http://dx.doi.org/10.1103/PhysRevA.98.012114, URL https://link.aps.org/doi/10.1103/PhysRevA.98.012114.

[317] D. Braun, G. Adesso, F. Benatti, R. Floreanini, U. Marzolino, M.W. Mitchell, S. Pirandola, Quantum-enhanced measurements without entanglement, Rev. Modern Phys. 90 (3) (2018) 035006, http://dx.doi.org/10.1103/RevModPhys.90.035006, URL https://link.aps.org/doi/10.1103/ RevModPhys.90.035006.

[318] P.C. Humphreys, M. Barbieri, A. Datta, I.A. Walmsley, Quantum enhanced multiple phase estimation, Phys. Rev. Lett. 111 (7) (2013) 070403, http://dx.doi.org/10.1103/PhysRevLett.111.070403, URL https://link.aps.org/doi/10.1103/PhysRevLett.111.070403.

[319] T. Baumgratz, A. Datta, Quantum enhanced estimation of a multidimensional field, Phys. Rev. Lett. 116 (3) (2016) 030801, http://dx.doi.org/ 10.1103/PhysRevLett.116.030801, URL https://link.aps.org/doi/10.1103/PhysRevLett.116.030801.

[320] L. Pezzè, M.A. Ciampini, N. Spagnolo, P.C. Humphreys, A. Datta, I.A. Walmsley, M. Barbieri, F. Sciarrino, A. Smerzi, Optimal measurements for simultaneous quantum estimation of multiple phases, Phys. Rev. Lett. 119 (13) (2017) 130504, http://dx.doi.org/10.1103/PhysRevLett.119. 130504, URL https://link.aps.org/doi/10.1103/PhysRevLett.119.130504.

[321] I. Apellaniz, I. Urizar-Lanz, Z. Zimborás, P. Hyllus, G. Tóth, Precision bounds for gradient magnetometry with atomic ensembles, Phys. Rev. A 97 (5) (2018) 053603, http://dx.doi.org/10.1103/PhysRevA.97.053603, URL https://link.aps.org/doi/10.1103/PhysRevA.97.053603. 
[322] L. Campos Venuti, M. Cozzini, P. Buonsante, F. Massel, N. Bray-Ali, P. Zanardi, Fidelity approach to the Hubbard model, Phys. Rev. B 78 (11) (2008) 115410, http://dx.doi.org/10.1103/PhysRevB.78.115410, URL https://link.aps.org/doi/10.1103/PhysRevB.78.115410.

[323] S. Garnerone, N.T. Jacobson, S. Haas, P. Zanardi, Fidelity approach to the disordered quantum XY model, Phys. Rev. Lett. 102 (5) (2009) 057205 , http://dx.doi.org/10.1103/PhysRevLett.102.057205, URL https://link.aps.org/doi/10.1103/PhysRevLett.102.057205.

[324] A.T. Rezakhani, D.F. Abasto, D.A. Lidar, P. Zanardi, Intrinsic geometry of quantum adiabatic evolution and quantum phase transitions, Phys. Rev. A 82 (1) (2010) 012321, http://dx.doi.org/10.1103/PhysRevA.82.012321, URL https://link.aps.org/doi/10.1103/PhysRevA.82.012321.

[325] L. Magazzù, A. Carollo, B. Spagnolo, D. Valenti, Quantum dissipative dynamics of a bistable system in the sub-Ohmic to super-Ohmic regime, J. Stat. Mech. Theory Exp. 2016 (5) (2016) 054016, http://dx.doi.org/10.1088/1742-5468/2016/05/054016, URL http://stacks.iop.org/1742$5468 / 2016 / \mathrm{i}=5 / \mathrm{a}=054016$ ? key=crossref.6a3bd3909efdcfdfd7b7d8c46d642d01.

[326] C. Guarcello, D. Valenti, A. Carollo, B. Spagnolo, Stabilization effects of dichotomous noise on the lifetime of the superconducting state in a long josephson junction, Entropy 17 (5) (2015) 2862-2875, http://dx.doi.org/10.3390/e17052862, URL http://www.mdpi.com/1099-4300/17/5/2862.

[327] A. Consiglio, A. Carollo, S.A. Zenios, A parsimonious model for generating arbitrage-free scenario trees, Quant. Finance 16 (2) (2016) 201-212, http://dx.doi.org/10.1080/14697688.2015.1114359, URL http://www.tandfonline.com/doi/full/10.1080/14697688.2015.1114359.

[328] M. Kolodrubetz, D. Sels, P. Mehta, A. Polkovnikov, Geometry and non-adiabatic response in quantum and classical systems, Phys. Rep. 697 (2017) 1-87, http://dx.doi.org/10.1016/j.physrep.2017.07.001, URL https://linkinghub.elsevier.com/retrieve/pii/S0370157317301989.

[329] A. Holevo, Probabilistic and Statistical Aspects of Quantum Theory, Edizioni della Normale, Pisa, 2011, http://dx.doi.org/10.1007/978-88-7642378-9, URL http://link.springer.com/10.1007/978-88-7642-378-9.

[330] M. Hayashi, K. Matsumoto, Asymptotic performance of optimal state estimation in qubit system, J. Math. Phys. 49 (10) (2008) 102101, http://dx.doi.org/10.1063/1.2988130, URL http://aip.scitation.org/doi/10.1063/1.2988130.

[331] J. Kahn, M. Guţă, Local asymptotic normality for finite dimensional quantum systems, Comm. Math. Phys. 289 (2) (2009) 597-652, http://dx.doi.org/10.1007/s00220-009-0787-3, URL http://link.springer.com/10.1007/s00220-009-0787-3.

[332] R.D. Gill, M.I. Guță, On asymptotic quantum statistical inference, in: M.H. Banerjee, M. Bunea, F. Huang, J. Koltchinskii, V. Maathuis (Eds.), From Probab. to Stat. Back High-Dimensional Model. Process. - A Festschrift Honor Jon A. Wellner, Vol. 9, Institute of Mathematical Statistics, Beachwood, Ohio, USA, 2013, pp. 105-127, http://dx.doi.org/10.1214/12-IMSCOLL909, URL http://projecteuclid.org/euclid.imsc/1362751183.

[333] K. Yamagata, A. Fujiwara, R.D. Gill, Quantum local asymptotic normality based on a new quantum likelihood ratio, Ann. Statist. 41 (4) (2013) 2197-2217, http://dx.doi.org/10.1214/13-AOS1147, URL http://projecteuclid.org/euclid.aos/1382547518.

[334] H. Cramér, Mathematical Methods of Statistics, Princeton University Press, 1946, p. 575.

[335] S.M. Kay, Fundamentals of Statistical Signal Processing, Prentice-Hall PTR, 1993.

[336] D.R. Cox, N. Reid, Parameter orthogonality and approximate conditional inference, J. R. Stat. Soc. Ser. B 49 (1987) 1-39, URL http: //www.jstor.org/stable/2345476.

[337] H.P. Robertson, The uncertainty principle, Phys. Rev. 34 (1) (1929) 163-164, http://dx.doi.org/10.1103/PhysRev.34.163, URL https://link.aps. org/doi/10.1103/PhysRev.34.163.

[338] R.A. Horn, C.R. Johnson, Matrix Analysis, Cambridge University Press, 2013, p. 643.

[339] D. Brody, N. Rivier, Geometrical aspects of statistical mechanics, Phys. Rev. E 51 (2) (1995) 1006-1011, http://dx.doi.org/10.1103/PhysRevE. 51.1006.

[340] A. Altland, B. Simons, Condensed Matter Field Theory, Cambridge University Press, Cambridge, 2006, http://dx.doi.org/10.1017/ CB09780511804236, URL http://ebooks.cambridge.org/ref/id/CBO9780511804236.

[341] S. Yim, T.S. Jones, Anomalous scaling behavior and surface roughening in molecular thin-film deposition, Phys. Rev. B 73 (16) (2006) 161305 http://dx.doi.org/10.1103/PhysRevB.73.161305, URL https://link.aps.org/doi/10.1103/PhysRevB.73.161305.

[342] R. Coldea, D.A. Tennant, E.M. Wheeler, E. Wawrzynska, D. Prabhakaran, M. Telling, K. Habicht, P. Smeibidl, K. Kiefer, Quantum criticality in an ising chain: Experimental evidence for emergent E 8 symmetry, Science 327 (5962) (2010) 177-180, http://dx.doi.org/10.1126/science.1180085, URL http://www.sciencemag.org/lookup/doi/10.1126/science.1180085.

[343] B. Lake, A.M. Tsvelik, S. Notbohm, D. Alan Tennant, T.G. Perring, M. Reehuis, C. Sekar, G. Krabbes, B. Büchner, Confinement of fractional quantum number particles in a condensed-matter system, Nat. Phys. (2010) http://dx.doi.org/10.1038/nphys1462.

[344] T.-H. Han, J.S. Helton, S. Chu, D.G. Nocera, J.A. Rodriguez-Rivera, C. Broholm, Y.S. Lee, Fractionalized excitations in the spin-liquid state of a kagome-lattice antiferromagnet, Nature 492 (7429) (2012) 406-410, http://dx.doi.org/10.1038/nature11659, URL http://www.nature.com/ articles/nature11659.

[345] P. Dai, Antiferromagnetic order and spin dynamics in iron-based superconductors, Rev. Modern Phys. 87 (3) (2015) 855-896, http://dx.doi org/10.1103/RevModPhys.87.855, URL https://link.aps.org/doi/10.1103/RevModPhys.87.855.

[346] M. Hälg, D. Hüvonen, N.P. Butch, F. Demmel, A. Zheludev, Finite-temperature scaling of spin correlations in a partially magnetized Heisenberg S=1/2 chain, Phys. Rev. B 92 (10) (2015) 104416, http://dx.doi.org/10.1103/PhysRevB.92.104416, URL https://link.aps.org/doi/10.1103/PhysRevB. 92.104416.

[347] L.V. Woodcock, Origins of thixotropy, Phys. Rev. Lett. 54 (14) (1985) 1513-1516, http://dx.doi.org/10.1103/PhysRevLett.54.1513, URL https: //link.aps.org/doi/10.1103/PhysRevLett.54.1513.

[348] D.C. Chrzan, M.J. Mills, Criticality in the plastic deformation of Ni3AI, Phys. Rev. Lett. 69 (19) (1992) 2795-2798, http://dx.doi.org/10.1103/ PhysRevLett.69.2795, URL https://link.aps.org/doi/10.1103/PhysRevLett.69.2795.

[349] V.A. Schweigert, I.V. Schweigert, A. Melzer, A. Homann, A. Piel, Plasma crystal melting: A nonequilibrium phase transition, Phys. Rev. Lett. 80 (24) (1998) 5345-5348, http://dx.doi.org/10.1103/PhysRevLett.80.5345, URL http://link.aps.org/doi/10.1103/PhysRevLett.80.5345.

[350] R.A. Blythe, M.R. Evans, Lee-Yang zeros and phase transitions in nonequilibrium steady states, Phys. Rev. Lett. 89 (8) (2002) 080601, http://dx.doi.org/10.1103/PhysRevLett.89.080601, URL https://link.aps.org/doi/10.1103/PhysRevLett.89.080601.

[351] S. Whitelam, L.O. Hedges, J.D. Schmit, Self-assembly at a nonequilibrium critical point, Phys. Rev. Lett. 112 (15) (2014) 155504, http: //dx.doi.org/10.1103/PhysRevLett.112.155504, URL https://link.aps.org/doi/10.1103/PhysRevLett.112.155504.

[352] X. Zhang, M. van Hulzen, D.P. Singh, A. Brownrigg, J.P. Wright, N.H. van Dijk, M. Wagemaker, Direct view on the phase evolution in individual LiFePO4 nanoparticles during Li-ion battery cycling, Nature Commun. 6 (May) (2015) 8333, http://dx.doi.org/10.1038/ncomms9333, URL http://www.nature.com/doifinder/10.1038/ncomms9333.

[353] S.U. Egelhaaf, P. Schurtenberger, Micelle-to-vesicle transition: A time-resolved structural study, Phys. Rev. Lett. 82 (13) (1999) 2804-2807, http://dx.doi.org/10.1103/PhysRevLett.82.2804, URL https://link.aps.org/doi/10.1103/PhysRevLett.82.2804.

[354] D. Marenduzzo, S.M. Bhattacharjee, A. Maritan, E. Orlandini, F. Seno, Dynamical scaling of the DNA unzipping transition, Phys. Rev. Lett. 88 (2) (2001) 028102, http://dx.doi.org/10.1103/PhysRevLett.88.028102, URL https://link.aps.org/doi/10.1103/PhysRevLett.88.028102.

[355] C. Barrett-Freeman, M.R. Evans, D. Marenduzzo, W.C.K. Poon, Nonequilibrium phase transition in the sedimentation of reproducing particles, Phys. Rev. Lett. 101 (10) (2008) 100602, http://dx.doi.org/10.1103/PhysRevLett.101.100602, URL https://link.aps.org/doi/10.1103/PhysRevLett. 101.100602.

[356] H.-J. Woo, A. Wallqvist, Nonequilibrium phase transitions associated with DNA replication, Phys. Rev. Lett. 106 (6) (2011) 060601, http: //dx.doi.org/10.1103/PhysRevLett.106.060601, URL https://link.aps.org/doi/10.1103/PhysRevLett.106.060601. 
[357] M. Mak, M.H. Zaman, R.D. Kamm, T. Kim, Interplay of active processes modulates tension and drives phase transition in self-renewing, motor-driven cytoskeletal networks, Nature Commun. 7 (May 2015) (2016) 10323, http://dx.doi.org/10.1038/ncomms10323, URL http://www. nature.com/doifinder/10.1038/ncomms10323.

[358] C. Battle, C.P. Broedersz, N. Fakhri, V.F. Geyer, J. Howard, C.F. Schmidt, F.C. MacKintosh, Broken detailed balance at mesoscopic scales in active biological systems, Science 352 (6285) (2016) 604-607, http://dx.doi.org/10.1126/science.aac8167, URL http://www.sciencemag.org/lookup/doi/ 10.1126/science.aac8167.

[359] M. Llas, P.M. Gleiser, J.M. López, A. Díaz-Guilera, Nonequilibrium phase transition in a model for the propagation of innovations among economic agents, Phys. Rev. E 68 (6) (2003) 066101, http://dx.doi.org/10.1103/PhysRevE.68.066101, URL https://link.aps.org/doi/10.1103/ PhysRevE.68.066101

[360] A. Baronchelli, L. Dall'Asta, A. Barrat, V. Loreto, Nonequilibrium phase transition in negotiation dynamics, Phys. Rev. E 76 (5) (2007) 051102, http://dx.doi.org/10.1103/PhysRevE.76.051102, URL https://link.aps.org/doi/10.1103/PhysRevE.76.051102.

[361] M. Scheffer, S.R. Carpenter, T.M. Lenton, J. Bascompte, W. Brock, V. Dakos, J. van de Koppel, I.A. van de Leemput, S.A. Levin, E.H. van Nes, M. Pascual, J. Vandermeer, Anticipating critical transitions, Science 338 (6105) (2012) 344-348, http://dx.doi.org/10.1126/science.1225244, URL http://www.sciencemag.org/cgi/doi/10.1126/science.1225244.

[362] G. Ódor, Universality classes in nonequilibrium lattice systems, Rev. Modern Phys. 76 (3) (2004) 663-724, http://dx.doi.org/10.1103/ RevModPhys.76.663, URL https://link.aps.org/doi/10.1103/RevModPhys.76.663.

[363] S. Lübeck, Universal scaling behavior of non-equilibrium phase transitions, Internat. J. Modern Phys. B 18 (31n32) (2004) 3977-4118, http://dx.doi.org/10.1142/S0217979204027748, URL http://www.worldscientific.com/doi/abs/10.1142/S0217979204027748.

[364] T. Prosen, B. Žunkovič, Exact solution of Markovian master equations for quadratic Fermi systems: thermal baths, open XY spin chains and non-equilibrium phase transition, New J. Phys. 12 (2) (2010) 025016, http://dx.doi.org/10.1088/1367-2630/12/2/025016, URL http: //stacks.iop.org/1367-2630/12/i=2/a=025016? key=crossref.27d4e8da79883b9ea2cc0dde04dc1523.

[365] M. Žnidarič, Relaxation times of dissipative many-body quantum systems, Phys. Rev. E 92 (4) (2015) 042143, http://dx.doi.org/10.1103/ PhysRevE.92.042143, URL https://link.aps.org/doi/10.1103/PhysRevE.92.042143.

[366] I. Bloch, J. Dalibard, W. Zwerger, Many-body physics with ultracold gases, Rev. Modern Phys. 80 (3) (2008) 885-964, http://dx.doi.org/10. 1103/RevModPhys.80.885, URL https://link.aps.org/doi/10.1103/RevModPhys.80.885.

[367] J.T. Barreiro, M. Müller, P. Schindler, D. Nigg, T. Monz, M. Chwalla, M. Hennrich, C.F. Roos, P. Zoller, R. Blatt, An open-system quantum simulator with trapped ions, Nature 470 (7335) (2011) 486-491, http://dx.doi.org/10.1038/nature09801, URL http://dx.doi.org/10.1038/nature09801www. ncbi.nlm.nih.gov/pubmed/21350481.

[368] P. Schindler, M. Müller, D. Nigg, J.T. Barreiro, E.A. Martinez, M. Hennrich, T. Monz, S. Diehl, P. Zoller, R. Blatt, Quantum simulation of dynamical maps with trapped ions, Nat. Phys. 9 (6) (2013) 361-367, http://dx.doi.org/10.1038/nphys2630, URL http://www.nature.com/articles/nphys2630.

[369] M.J. Hartmann, F.G.S.L. Brandao, M.B. Plenio, Strongly interacting polaritons in coupled arrays of cavities, Nat. Phys. 2 (12) (2006) 849-855, http://dx.doi.org/10.1038/nphys462.

[370] A.D. Greentree, C. Tahan, J.H. Cole, L.C.L. Hollenberg, Quantum phase transitions of light, Nat. Phys. 2 (12) (2006) 856-861, http://dx.doi.org/ 10.1038/nphys466, URL http://www.nature.com/articles/nphys466.

[371] D.G. Angelakis, M.F. Santos, S. Bose, Photon-blockade-induced mott transitions and XY spin models in coupled cavity arrays, Phys. Rev. A 76 (3) (2007) 031805, http://dx.doi.org/10.1103/PhysRevA.76.031805, URL https://link.aps.org/doi/10.1103/PhysRevA.76.031805.

[372] D.L. Underwood, W.E. Shanks, J. Koch, A.A. Houck, Low-disorder microwave cavity lattices for quantum simulation with photons, Phys. Rev. A 86 (2) (2012) 023837, http://dx.doi.org/10.1103/PhysRevA.86.023837, URL https://link.aps.org/doi/10.1103/PhysRevA.86.023837.

[373] A.A. Houck, H.E. Türeci, J. Koch, On-chip quantum simulation with superconducting circuits, Nat. Phys. 8 (4) (2012) 292-299, http: //dx.doi.org/10.1038/nphys2251, URL http://www.nature.com/articles/nphys2251.

[374] J. Raftery, D. Sadri, S. Schmidt, H.E. Türeci, A.A. Houck, Observation of a dissipation-induced classical to quantum transition, Phys. Rev. X 4 (3) (2014) 031043, http://dx.doi.org/10.1103/PhysRevX.4.031043, URL https://link.aps.org/doi/10.1103/PhysRevX.4.031043.

[375] H. Weimer, M. Müller, I. Lesanovsky, P. Zoller, H.P. Büchler, A rydberg quantum simulator, Nat. Phys. 6 (5) (2010) 382-388, http://dx.doi.org/ 10.1038/nphys 1614, URL http://www.nature.com/articles/nphys1614.

[376] Y.O. Dudin, L. Li, F. Bariani, A. Kuzmich, Observation of coherent many-body rabi oscillations, Nat. Phys. 8 (11) (2012) 790-794, http: //dx.doi.org/10.1038/nphys2413, URL http://www.nature.com/articles/nphys2413.

[377] J. Eisert, M. Friesdorf, C. Gogolin, Quantum many-body systems out of equilibrium, Nat. Phys. 11 (2) (2015) 124-130, http://dx.doi.org/10. 1038/nphys3215, URL http://www.nature.com/doifinder/10.1038/nphys3215.

[378] G. Lindblad, On the generators of quantum dynamical semigroups, Comm. Math. Phys. 48 (1976) 119-130.

[379] F. Verstraete, M.M. Wolf, J. Ignacio Cirac, Quantum computation and quantum-state engineering driven by dissipation, Nat. Phys. 5 (9) (2009) 633-636, http://dx.doi.org/10.1038/nphys1342, URL http://www.nature.com/articles/nphys1342.

[380] M. Höning, M. Moos, M. Fleischhauer, Critical exponents of steady-state phase transitions in fermionic lattice models, Phys. Rev. A 86 (1) (2012) 013606, http://dx.doi.org/10.1103/PhysRevA.86.013606, URL https://link.aps.org/doi/10.1103/PhysRevA.86.013606.

[381] B. Horstmann, J.I. Cirac, G. Giedke, Noise-driven dynamics and phase transitions in fermionic systems, Phys. Rev. A 87 (1) (2013) 012108 , http://dx.doi.org/10.1103/PhysRevA.87.012108, URL https://link.aps.org/doi/10.1103/PhysRevA.87.012108.

[382] C.-E. Bardyn, M.A. Baranov, C.V. Kraus, E. Rico, A. mamolu, P. Zoller, S. Diehl, Topology by dissipation, New J. Phys. 15 (8) (2013) 085001, http://dx.doi.org/10.1088/1367-2630/15/8/085001, URL http://stacks.iop.org/1367-2630/15/i=8/a=085001?key=crossref dbfc132c3a50871fe00070d6baa253c4.

[383] R.P. Feynman, Simulating physics with computers, Internat. J. Theoret. Phys. 21 (6/7) (1982) 467, http://dx.doi.org/10.1007/BF02650179, URL http://link.springer.com/10.1007/BF02650179.

[384] S. Lloyd, Universal quantum simulators, Science (80-. ). 273 (5278) (1996) 1073-1078, http://dx.doi.org/10.1126/science.273.5278.1073, URL http://www.sciencemag.org/cgi/doi/10.1126/science.273.5278.1073.

[385] I. Bloch, J. Dalibard, S. Nascimbène, Quantum simulations with ultracold quantum gases, Nat. Phys. 8 (4) (2012) 267-276, http://dx.doi.org/ 10.1038/nphys2259, URL http://www.nature.com/articles/nphys2259.

[386] R. Blatt, C.F. Roos, Quantum simulations with trapped ions, Nat. Phys. 8 (4) (2012) 277-284, http://dx.doi.org/10.1038/nphys2252, URL http://www.nature.com/articles/nphys2252.

[387] A. Aspuru-Guzik, P. Walther, Photonic quantum simulators, Nat. Phys. 8 (4) (2012) 285-291, http://dx.doi.org/10.1038/nphys2253.

[388] V. Bach, E.H. Lieb, J.P. Solovej, Generalized Hartree-Fock theory and the Hubbard model, J. Stat. Phys. 76 (1-2) (1994) 3-89, http://dx.doi.org/ 10.1007/BF02188656, URL http://link.springer.com/10.1007/BF02188656.

[389] T. Prosen, Third quantization: a general method to solve master equations for quadratic open Fermi systems, New J. Phys. 10 (4) (2008) 043026, http://dx.doi.org/10.1088/1367-2630/10/4/043026, URL http://stacks.iop.org/1367-2630/10/i=4/a=043026?key=crossref b000d5ff6bcc288ed499ccbcf202d73d.

[390] T. Prosen, Spectral theorem for the Lindblad equation for quadratic open fermionic systems, J. Stat. Mech. Theory Exp. 2010 (07) (2010) P07020, http://dx.doi.org/10.1088/1742-5468/2010/07/P07020, URL http://stacks.iop.org/1742-5468/2010/i=07/a=P07020?key=crossref dbdde3357621be1587fb254ddd3f9886. 
[391] B. Žunkovič, T. Prosen, Explicit solution of the Lindblad equation for nearly isotropic boundary driven XY spin $1 / 2$ chain, J. Stat. Mech. Theory Exp. 2010 (08) (2010) P08016, http://dx.doi.org/10.1088/1742-5468/2010/08/P08016, URL http://stacks.iop.org/1742-5468/2010/i=08/ $\mathrm{a}=$ P08016? key=crossref.f270fcbdc5b226375cc426faf937b2f8.

[392] J.-P. Blaizot, G. Ripka, Quantum Theory of Finite Systems, MIT Press, 1986, p. 657

[393] M. Žnidarič, Solvable quantum nonequilibrium model exhibiting a phase transition and a matrix product representation, Phys. Rev. E 83 (1) (2011) 011108, http://dx.doi.org/10.1103/PhysRevE.83.011108, URL https://link.aps.org/doi/10.1103/PhysRevE.83.011108.

[394] Z. Cai, T. Barthel, Algebraic versus exponential decoherence in dissipative many-particle systems, Phys. Rev. Lett. 111 (15) (2013) 150403, http://dx.doi.org/10.1103/PhysRevLett.111.150403, URL https://link.aps.org/doi/10.1103/PhysRevLett.111.150403.

[395] A. Carollo, I. Fuentes-Guridi, M.F. Santos, V. Vedral, Geometric phase in open systems, Phys. Rev. Lett. 90 (16) (2003) 160402, http: //dx.doi.org/10.1103/PhysRevLett.90.160402, URL https://link.aps.org/doi/10.1103/PhysRevLett.90.160402.

[396] A. Carollo, I. Fuentes-Guridi, M.F. Santos, V. Vedral, Spin-1/2 geometric phase driven by decohering quantum fields, Phys. Rev. Lett. 92 (2) (2004) 020402, http://dx.doi.org/10.1103/PhysRevLett.92.020402, URL https://link.aps.org/doi/10.1103/PhysRevLett.92.020402.

[397] E. Ercolessi, M. Schiavina, Symmetric logarithmic derivative for general n-level systems and the quantum Fisher information tensor for threelevel systems, Phys. Lett. A 377 (34-36) (2013) 1996-2002, http://dx.doi.org/10.1016/j.physleta.2013.06.012, URL https://linkinghub.elsevier. com/retrieve/pii/S0375960113005872.

[398] A. Monras, Phase space formalism for quantum estimation of Gaussian states, 2013, URL http://arxiv.org/abs/1303.3682.

[399] Z. Jiang, Quantum Fisher information for states in exponential form, Phys. Rev. A 89 (3) (2014) 1-6, http://dx.doi.org/10.1103/PhysRevA.89. 032128.

[400] D.J. Thouless, Topological Quantum Numbers in Nonrelativistic Physics, World Scientific, Singapore, London, 1998.

[401] R.M. Wilcox, Exponential operators and parameter differentiation in quantum physics, J. Math. Phys. 8 (4) (1967) 962-982, http://dx.doi.org/ 10.1063/1.1705306, URL http://aip.scitation.org/doi/10.1063/1.1705306. 